\title{
The Evolution of Compact Binary Star Systems
}

\author{
Konstantin A. Postnov \\ Moscow M.V. Lomonosov State University \\ Sternberg Astronomical Institute \\ 13 Universitetskij Pr. \\ 119992 Moscow \\ Russia \\ email: pk@sai.msu.ru \\ http://www.sai.msu.ru \\ Lev R. Yungelson \\ Institute of Astronomy of the Russian Academy of Sciences \\ 48 Pyatnitskaya St. \\ 119017 Moscow \\ Russia \\ email: lry@inasan.ru \\ http://www.inasan.ru
}

Accepted: 17 March 2014

Published: 5 May 2014

(Update of lrr-2006-6)

\begin{abstract}
We review the formation and evolution of compact binary stars consisting of white dwarfs (WDs), neutron stars (NSs), and black holes (BHs). Mergings of compact-star binaries are expected to be the most important sources for forthcoming gravitational-wave (GW) astronomy. In the first part of the review, we discuss observational manifestations of close binaries with $\mathrm{NS}$ and/or BH components and their merger rate, crucial points in the formation and evolution of compact stars in binary systems, including the treatment of the natal kicks, which NSs and $\mathrm{BHs}$ acquire during the core collapse of massive stars and the common envelope phase of binary evolution, which are most relevant to the merging rates of NS-NS, NS-BH and BH-BH binaries. The second part of the review is devoted mainly to the formation and evolution of binary WDs and their observational manifestations, including their role as progenitors of cosmologically-important thermonuclear SN Ia. We also consider AM CVn-stars, which are thought to be the best verification binary GW sources for future low-frequency GW space interferometers.
\end{abstract}

Keywords: Astrophysics, Binary systems, Gravitational-wave sources, Supernovae, Neutron stars, Black holes, White dwarfs, AM CVn stars

This review is licensed under a Creative Commons Attribution-Non-Commercial 3.0 Germany License. http://creativecommons . org/licenses/by-nc/3.0/de/ 


\section{Imprint / Terms of Use}

Living Reviews in Relativity is a peer reviewed open access journal published by the Max Planck Institute for Gravitational Physics, Am Mühlenberg 1, 14476 Potsdam, Germany. ISSN 1433-8351.

This review is licensed under a Creative Commons Attribution-Non-Commercial 3.0 Germany License: http://creativecommons.org/licenses/by-nc/3.0/de/. Figures that have been previously published elsewhere may not be reproduced without consent of the original copyright holders.

Because a Living Reviews article can evolve over time, we recommend to cite the article as follows:

Konstantin A. Postnov and Lev R. Yungelson,

"The Evolution of Compact Binary Star Systems",

Living Rev. Relativity, 17, (2014), 3. URL (accessed < date>):

http://www.livingreviews.org/lrr-2014-3

The date given as $<$ date $>$ then uniquely identifies the version of the article you are referring to. 


\section{Article Revisions}

Living Reviews supports two ways of keeping its articles up-to-date:

Fast-track revision. A fast-track revision provides the author with the opportunity to add short notices of current research results, trends and developments, or important publications to the article. A fast-track revision is refereed by the responsible subject editor. If an article has undergone a fast-track revision, a summary of changes will be listed here.

Major update. A major update will include substantial changes and additions and is subject to full external refereeing. It is published with a new publication number.

For detailed documentation of an article's evolution, please refer to the history document of the article's online version at http://www. livingreviews.org/lrr-2014-3.

5 May 2014: Major revision, updated and expanded.

References are updated by 2007 -early-2014 publications, some outdated items removed. The reference list now contains 887 items instead of 475. The new version of the review has 39 figures.

In Section 1, stellar evolution is outlined in more detail, special attention is paid to electron-capture supernovae. More attention than before is paid to uncertainties in the estimates of the ranges of precursors of white dwarfs, neutron stars and black holes and their masses.

In Section 2, a subsection (2.3) on black holes in binaries is added and an independent estimate of black-hole/neutron-star binary coalescence rate is given.

In Section 3, a subsection on mass transfer between binary components is added, discussion of common envelopes is expanded.

In Section 7, the discussion of a scenario for the formation of short-period binaries is appended by a more detailed description of "intermediate" stages, like post-common-envelope binaries, the discussion of properties of binary WD is expanded, the comparison of results of population synthesis calculations for close WD with observations is updated, thanks to a significant increase in the number of observed objects. A much extended comparison of expectations from scenarios for single-degenerate and double-degenerate SNe Ia (including violent mergers) is presented.

In Section 8 all published information on double-degenerates with measured periods and estimates of masses of components and some subgiant + [white dwarf] systems is summarized (up to February 2014).

In Section 9 more attention than previously is paid to the initial stages of mass exchange in the "white dwarf" channel of formation of AM CVn stars and to the comparison of "white dwarf" and "helium star" channels. Final stages of the evolution of AM CVn stars and SN .Ia are discussed.

In Section 10 the GW-signals of detached and interacting double-degenerates are compared in more detail, as well as their detection probabilities with planned "short-arm" (eLISA) interferometer.

The former section on the overlap of EM and GW-signals from AM CVn stars is deleted because of the (temporary?) cancellation of the LISA-mission. 


\section{Contents}

1 Introduction $\quad 7$

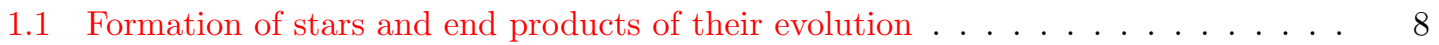

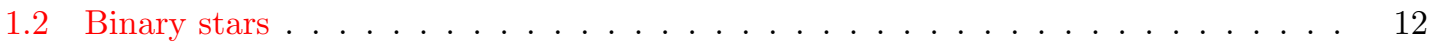

2 Observations of Double Compact Stars $\quad 14$

2.1 Compact binaries with neutron stars . . . . . . . . . . . . . . . . . . . 14

2.2 How frequent are NS binary coalescences? . . . . . . . . . . . . . . . . . . . . 16

2.3 Black holes in binary systems . . . . . . . . . . . . . . . . . . . . 17

2.4 A model-independent upper limit on the $\mathrm{BH}-\mathrm{BH} / \mathrm{BH}-\mathrm{NS}$ coalescence rate $\ldots . . . \quad 19$

3 Basic Principles of the Evolution of Binary Stars 21

3.1 Keplerian binary system and radiation back reaction . . . . . . . . . . . . . . 21

3.1 .1 Keplerian motion . . . . . . . . . . . . . . . . . . . . 21

3.1 .2 Gravitational radiation from a binary . . . . . . . . . . . . . 22

3.1.3 Energy and angular momentum loss . . . . . . . . . . . . . . . . 23

3.1.4 Binary coalescence time . . . . . . . . . . . . . . . . . . . 24

3.1 .5 Magnetic stellar wind . . . . . . . . . . . . . . . . . 25

3.2 Mass exchange in close binaries . . . . . . . . . . . . . . . . 26

3.3 Mass transfer modes and mass and angular momentum loss in binary systems . . . 28

3.3 .1 Conservative accretion . . . . . . . . . . . . . . . . . . . . 30

3.3.2 The Jeans (fast wind) mode . . . . . . . . . . . . . . . . . . . . 30

3.3 .3 Isotropic re-emission . . . . . . . . . . . . . . . . . . . . . . . 31

3.4 Supernova explosion . . . . . . . . . . . . . . . . . . . . . . . . . 32

3.5 Kick velocity of neutron stars . . . . . . . . . . . . . . . . . . 33

3.5.1 Effect of the kick velocity on the evolution of a binary system . . . . . . . 35

3.6 Common envelope stage . . . . . . . . . . . . . . . . . . . 36

3.6.1 Formation of the common envelope . . . . . . . . . . . . . 36

3.6 .2 "Alpha"-formalism . . . . . . . . . . . . . . . . . . . . . . . . 38

3.6.3 "Gamma"-formalism . . . . . . . . . . . . . . . . . . . . . . . . . . 39

3.7 Other notes on the $\mathrm{CE}$ problem . . . . . . . . . . . . . . . . . 41

4 Evolutionary Scenario for Compact Binaries with Neutron Star or Black Hole Components $\quad \mathbf{4 2}$

4.1 Compact binaries with neutron stars . . . . . . . . . . . . . . . . . 42

4.2 Black-hole-formation parameters . . . . . . . . . . . . . . . 48

5 Formation of Double Compact Binaries $\quad \mathbf{5 0}$

5.1 Analytical estimates . . . . . . . . . . . . . . . . . 50

5.2 Population synthesis results . . . . . . . . . . . . . . . . . . . 51

6 Detection Rates $\quad \mathbf{5 4}$

7 Short-Period Binaries with White-Dwarf Components $\quad \mathbf{5 7}$

7.1 Formation of compact binaries with white dwarfs . . . . . . . . . . 58

7.1.1 Post-common envelope binaries . . . . . . . . . . . . . . . . . . 60

7.1 .2 Cataclysmic variables . . . . . . . . . . . . . . . . . 60

7.2 White-dwarf binaries . . . . . . . . . . . . . . . . . . . . . . 62

7.3 Type Ia supernovae . . . . . . . . . . . . . . . . . . . . . . . . . . . . . . 64

7.3 .1 Single-degenerate scenario . . . . . . . . . . . . . . . 66 
7.3.2 Merger of white dwarfs and the double-degenerate scenario for SN Ia . . . 72

7.4 Ultra-compact X-ray binaries . . . . . . . . . . . . . . . . . 78

8 Observations of Double-Degenerate Systems $\quad \mathbf{7 9}$

8.1 Detached white dwarf and subdwarf binaries . . . . . . . . . . . . . 79

9 Evolution of Interacting Double-Degenerate Systems $\quad \mathbf{8 2}$

9.1 "Double-degenerate family" of AM CVn stars . . . . . . . . . . . . . . . . 83

9.2 "Helium-star family" of AM CVn stars . . . . . . . . . . . . . . . . . . . 88

9.3 Final stages of evolution of interacting double-degenerate systems . . . . . . . . . 91

10 Gravitational Waves from Compact Binaries with White-Dwarf Components 92

11 AM CVn-Type Stars as Sources of Optical and X-Ray Emission 105

12 Conclusions $r$

$\begin{array}{lr}\text { References } & 111\end{array}$

\section{List of Tables}

1 Types of compact remnants of single stars. . . . . . . . . . . . . . . . . 11

2 Observed parameters of neutron star binaries. . . . . . . . . . . . . . . . . 15

3 Derived parameters of neutron star binaries. . . . . . . . . . . . . . . . 15

4 Observed parameters of HMXB with black holes. . . . . . . . . . . . . . . 19

5 Comparison of Fe-Core Collapse and e-Capture Supernovae. . . . . . . . . . . . . . 44

6 Examples of the estimates for Galactic merger rates of relativistic binaries calculated under different assumptions on the parameters entering population synthesis. . . . 52

7 Properties of companions to exploding WD. . . . . . . . . . . . . . . . . 72

8 Current birth rates and merger rates per year for Galactic disk binaries containing two compact objects and their total number in the Galactic disk. . . . . . . . . . . 95

9 Detectable sources. . . . . . . . . . . . . . . . . . . . . . 99 



\section{Introduction}

Close binaries consisting of two compact stellar remnants - white dwarfs (WDs), neutron stars (NSs) or black holes (BHs) are considered primary targets of the forthcoming field of gravitational wave (GW) astronomy (see, for a review, [758, 252, 673, 672]), since their orbital evolution is entirely controlled by the emission of gravitational waves and leads to ultimate coalescence (merger) and possible explosive disruption of the components. Emission of gravitational waves accompanies the latest stages of evolution of stars and manifests instabilities in relativistic objects [10, 13]. Close compact binaries can thus serve as testbeds for theories of gravity [221]. The NS(BH) binary mergers that release $\sim 10^{52}$ erg as GWs $[113,114]$ should be the brightest GW events in the $10-1000 \mathrm{~Hz}$ frequency band of the existing or future ground-based GW detectors like LIGO [23], VIRGO [6], GEO600 [635], KAGRA(LCGT) [728] (see also [642] for a review of the current state of existing and 2nd- and 3rd-generation ground-based detectors). Mergers of NS(BH) binaries can be accompanied by the release of a huge amount of electromagnetic energy in a burst and manifest themselves as short gamma-ray bursts (GRBs). A lot of observational support for NS-NS/NS-BH mergers as sources of short GRBs have been obtained (see, e.g., studies of short GRB locations in the host galaxies [204, 751] and references therein). As well, relativistic jets, associated with GRB of any nature may be sources of GW in the ground-based detectors range [50].

WD binaries, especially those observed as AM CVn-stars and ultracompact X-ray binaries (UCXB), are potential GW sources within the frequency band $\left(10^{-4}-1\right) \mathrm{Hz}$ of the space GW interferometers like (the currently cancelled) LISA [187], ${ }^{1}$ NGO (eLISA) [10, 11], DEGIGO [857] and other proposed or planned low-frequency GW detectors [122, 40]. At the moment, eLISA is selected for the third large-class mission in ESA's Cosmic Vision science program (L3). Its first step should be the launch of ESA's LISA Pathfinder (LPF) mission in 2015; the launch of eLISA itself is currently planned for $2034 .^{2}$

WD binary mergers are among the primary candidate mechanisms for type Ia supernovae (SNe Ia) explosions. The NIR magnitudes of the latter are considered as the best "standard candles" [26] and, in this guise, are crucial in modern cosmological studies [638, 578]. Further improvements in the precision of standardization of SNe Ia fluxes is possible, e.g., by account of their environmental dependence $[640,641,820]$. On the other hand, usually, as an event beneath the "standard candle", an explosion of a non-rotating WD with the Chandrasekhar mass $\left(\simeq 1.38 M_{\odot}\right)$ is considered. Rotation of progenitors may increase the critical mass and make SNe Ia less reliable for cosmological use [154, 155].

SNe Ia are suggested to be responsible for the production of about $50 \%$ of all heavy elements in the Universe [761]. Mergers of WD binaries are, probably, one of the main mechanisms of the formation of massive $\left(>0.8 M_{\odot}\right)$ WDs and WDs with strong magnetic fields (see, e.g., [322, 392] for recent studies and review of earlier work).

A comparison of SN Ia rates (for the different models of their progenitors) with observations may, in principle, shed light on both the star formation history and on the nature of the progenitors (see, e.g., [663, 873, 453, 874, 871, 250, 457, 249, 458]).

Compact binaries are the end products of the evolution of stellar binaries, and the main purpose of the present review is to describe the astrophysical knowledge of their formation and evolution. We shall discuss the present situation with the main parameters determining their evolution and the rates of coalescence of NS/BH binaries and WDs.

The problem is to evaluate as accurately as possible (i) the physical parameters of the coalescing binaries (masses of the components and, if possible, their spins, magnetic fields, etc.) and (ii) the

\footnotetext{
1 http://lisa.gsfc.nasa.gov/cosmic_vision_changes.html, http://sci.esa.int/science-e/www/object/ index. cfm?fobjectid $=48661$

2 https://www.elisascience.org/news/top-news/gravitationaluniverseselectedas13
} 
occurrence rate of mergers in the Galaxy and in the local Universe. Masses of NSs in binaries are known with a rather good accuracy of $10 \%$ or better from, e.g., pulsar studies [760, 367]; see also $[410,550]$ for recent summaries of NS mass measurements.

The case is not so good with the rate of coalescence of relativistic stellar binaries. Unfortunately, there is no way to derive it from first principles - neither the formation rate of the progenitors for compact binaries nor stellar evolution are known well enough. However, the situation is not completely hopeless, especially in the case of NS binary systems. The natural appearance of rotating NSs with magnetic fields as radio pulsars allows one to search for binary pulsars with a secondary compact companion using powerful methods of modern radio astronomy (for example, in dedicated pulsar surveys, such as the Parkes multi-beam pulsar survey [455, 192]).

Based on observational statistics of Galactic pulsar binaries with NS companions, one can evaluate the Galactic rate of NS binary formation and merging [586, 505]. On the other hand, a direct simulation of the evolution of population of binaries in the Galaxy (the population synthesis method) can also predict the formation and merger rates of close compact binaries as a function of (numerous) parameters of stellar formation and evolution. Both kinds of estimates are plagued by badly constrained parameters or selection effects, but it is, nevertheless, encouraging that most likely Galactic rates of events obtained in two ways currently differ by a factor of $\approx 3$ only: $80 / \mathrm{Myr}$ from observations and 30/Myr from population synthesis; see [456] for a recent review of observational and theoretical estimates, also Section 6 .

No BH or NS + BH binary systems have been found so far, so merger rates of compact binaries with BHs have been evaluated as yet only from population synthesis studies.

\subsection{Formation of stars and end products of their evolution}

Let us briefly remind the key facts about star formation and evolution. Approximately $6 \%$ of the baryonic matter in the Universe is confined to stars [217]. Recent observational data suggests that, first, long thin filaments form inside molecular clouds and, next, these filaments fragment into protostellar cores due to gravitational instability, if their mass-per-unit length exceeds a certain threshold [14]. An object may be called a "star" if it is able to generate energy by nuclear fusion at a level sufficient to halt the contraction [394]. For solar chemical composition, $M_{\min } \approx 0.075 M_{\odot}$ and $M_{\min }$ increases if stellar metallicity is lower than solar [81]. Currently, among observed stars, the lowest dynamically determined mass has component $\mathrm{C}$ of a triple system $\mathrm{AB}$ Dor: $(0.090 \pm$ $0.005) M_{\odot}[115]$.

The maximum mass of the star is set by the proximity of the luminosity to the Eddington limit and pulsational instability. For solar chemical composition, this limit is close to $1000 M_{\odot}[38$, 880]. ${ }^{3}$ But conditions of stellar formation, apparently, define a much lower mass limit. Currently, components of a $P_{\text {orb }}=3.77$ day eclipsing binary NGC3603-A1 have maximum dynamicallymeasured masses: $(116 \pm 31) M_{\odot}$ and $(89 \pm 16) M_{\odot}$ [683]. Since both stars are slightly-evolved mainsequence objects (WN6h), subject to severe-stellar-wind mass loss, their inferred initial masses could be higher: $148_{-27}^{+40} M_{\odot}$ and $106_{-15}^{+92} M_{\odot}$ [125]. Indirect evidence, based on photometry and spectral analysis suggests the possible existence of $(200-300) M_{\odot}$ stars; see [813] for references and discussion.

For the metal-free stars that formed first in the Universe (the so-called Population III stars), the upper mass limit is rather uncertain. For example, it can be below $100 M_{\odot}[4,745]$, because of the competition of accretion onto first formed compact core and nuclear burning and influence of UV-irradiation from nearby protostars. On the other hand, masses of Population III stars could be much higher due to the absence of effective coolants in the primordial gas. Then, their masses

3 The existence of such high-mass very short-living objects is deemed possible due to runaway stellar collisions in dense young compact stellar clusters [605]; for the prospects of observing them see, e.g., [564]. 
could be limited by pulsations, though, the upper mass limit remains undefined [703] and masses up to $\simeq 1000 M_{\odot}$ are often inferred in theoretical studies.

The initial mass function (IMF) of main-sequence stars can be approximated by a power-law $d N / d M \sim M^{-\beta}[669]$ and most simply may be presented taking $\beta \approx 1.3$ for $0.07 \lesssim M / M_{\odot} \lesssim 0.5$ and $\beta \approx 2.3$ for $M / M_{\odot} \gtrsim 0.5$ [830]. Note, there are also claims that for the most massive stars $\left(M \gtrsim 7 M_{\odot}\right)$ the IMF is much steeper - up to $\beta=3.8 \pm 0.5$, see [403] and references therein. Estimates of the current star-formation rate (SFR) in the Galaxy differ depending on the method, but most at present converge to $\simeq 2 M_{\odot} \mathrm{yr}^{-1}[108,353,537]$. This implies that in the past the Galactic SFR was much higher. For the most recent review of observations and models pertinent to the star-formation process, the origin of the initial mass function, and the clustering of stars, see [389].

The evolution of a single star and the nature of its compact remnant are determined by the main-sequence mass $M_{0}$ and chemical composition. If $M_{0}$ is lower than the minimum mass of stars that ignite carbon in the core, $M_{\mathrm{up}}$, after exhausting the hydrogen and helium in its core, a carbon-oxygen WD forms. If $M_{0}$ exceeds a certain limiting $M_{\text {mas }}$, the star proceeds to form an iron core, which collapses into a NS or a BH. In the stars of intermediate range between $M_{\text {up }}$ and $M_{\text {mas }}$ (called "super-AGB stars" ${ }^{4}$ ) carbon ignites in a partially-degenerate core and converts its matter (completely or partially) into an oxygen-neon mixture. As conjectured by Paczyński and Ziolkowski [557], the TP-AGB stage of the evolution of stars ${ }^{5}$ terminates when shells with positive specific binding energy $\varepsilon_{\text {bind }}=u_{\text {int }}+\varepsilon_{\text {grav }}$ appear in the envelope of the star. In the latter expression, the internal energy term $u_{\text {int }}$ accounts for ideal gas, radiation, ionisation, dissociation and electron degeneracy, as suggested by Han et al. [266]. The upper limit on masses of model precursors of $\mathrm{CO}$ white dwarfs depends primarily on stellar metallicity, on the treatment of mixing in stellar interiors, on accepted rates of mass loss during the AGB and on more subtle details of the models, see, e.g., $[278,279,338]$. Modern studies suggest that for solar metallicity models it does not exceed $1.2 M_{\odot}[412]$.

As concerns more massive stars, if, due to He-burning in the shell, the mass of the core reaches $\approx 1.375 M_{\odot}$, electron captures by ${ }^{24} \mathrm{Mg}$ and ${ }^{20} \mathrm{Ne}$ ensue and the core collapses to a neutron star producing an "electron-capture supernova" (ECSN) [488]). Otherwise, an ONe WD forms. ${ }^{6}$ Figure 1 shows, as an example, the endpoints of stellar evolution for intermediate mass stars, computed by Siess $[707,708] .{ }^{7}$ Note, results of computations very strongly depend on still uncertain rates of nuclear burning, the treatment of convection, the rate of assumed stellar-wind mass loss, which is poorly known from observations for the stars in this transition mass range, as well as on other subtle details of the stellar models (see discussion in [599, 325, 98, 749]). For instance, zero-age main sequence (ZAMS) masses of solar-composition progenitors of ECSN found by Poelarends et al. [599] are $M_{0} \approx(9.0-9.25) M_{\odot}{ }^{8}$ In [295], the lower mass limit for core-collapse SN at solar metallicity is found to be equal to $9.5 M_{\odot}$. According to Jones et al. [326], an $M_{\text {ZAMS }}=8.8 M_{\odot}$ star experiences ECSN, while a $9.5 M_{\odot}$ star evolves to Fe-core collapse. Takahashi et al. [749] find that a $M_{\mathrm{ZAMS}}=10.4 M_{\odot}$ solar metallicity star explodes as ECSN, while an $M_{\mathrm{ZAMS}}=11 M_{\odot}$ star experiences Fe-core collapse, consistent with Siess' results.

ECSN eject only a small amount of $\mathrm{Ni}\left(<0.015 M_{\odot}\right)$, and it was suggested that they may be

\footnotetext{
${ }^{4}$ AGB - Asymptotic Giant Branch on the Hertzsprung-Russell diagram

5 TP-AGB - thermally pulsing AGB, the late stage of the AGB evolution, characterized by the development of strong pulsations leading ultimately to the ejection of the entire envelope of the red giant star.

${ }^{6}$ Yet another possibility for the formation of neutron stars exists: if a sufficiently cold white dwarf close to critical mass accretes matter, the time scale of its gravitational collapse allows neutronization before the onset of pycnonuclear reactions [85].

${ }^{7}$ See these papers for detailed references and discussion of earlier studies.

${ }^{8}$ Results obtained by the same group for lower metallicities may be found in Poelarend's PhD thesis at http: //www. astro.uni-bonn.de/ nlanger/siu_web/pre.html.
} 


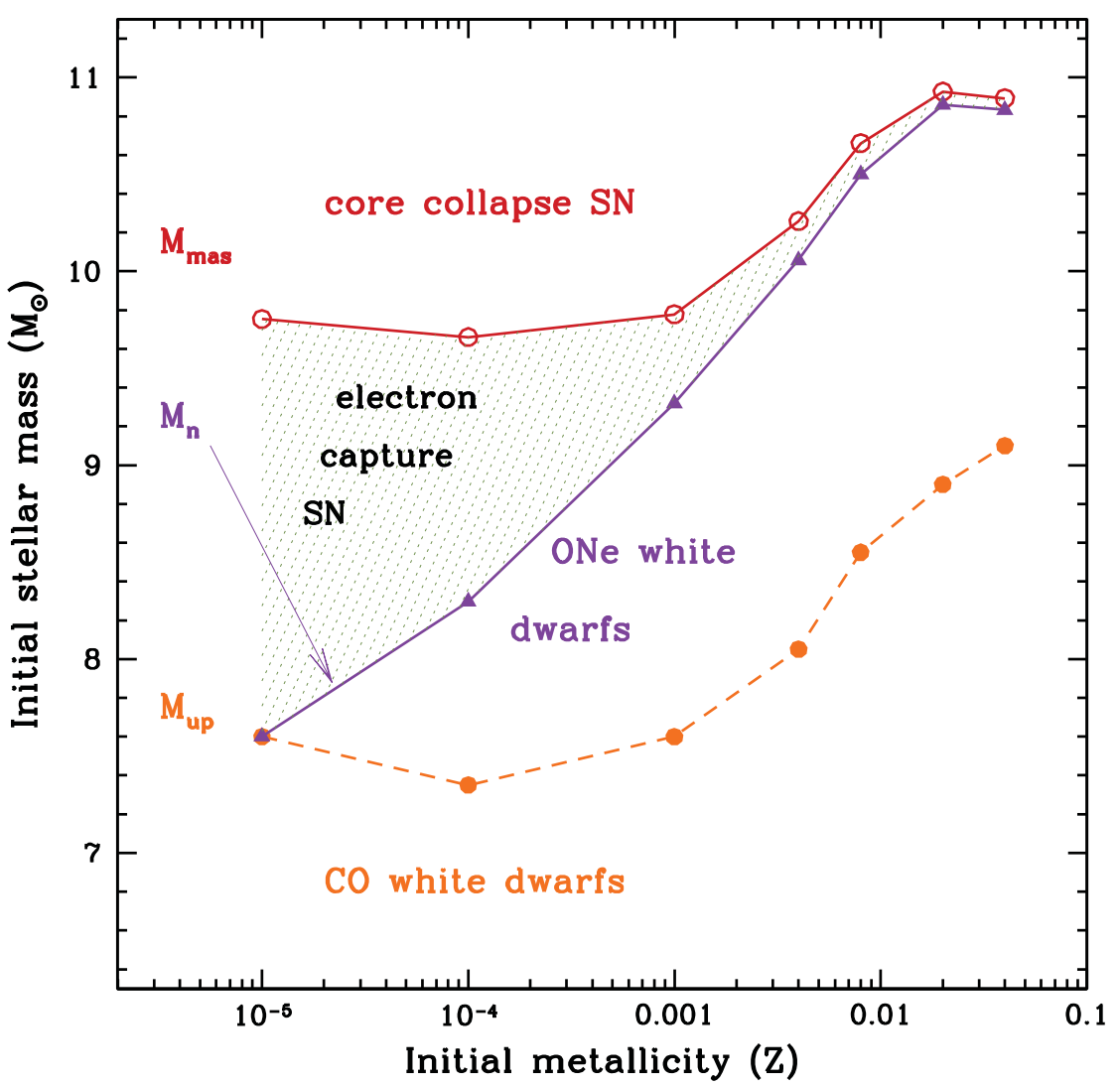

Figure 1: Endpoints of evolution of moderate-mass nonrotating single stars depending on initial mass and metallicity. Image reproduced with permission from [709], copyright by IAU.

identified with some subluminous type II-P supernovae [366]. Tominaga et al. [764] were able to simulate ECSN starting from first principles and to reproduce their multicolor light curves. They have shown that observed features of SN 1054 (see [741]) share the predicted characteristics of ECSN.

We should enter a caveat that in [412] it is claimed that opacity-related instability at the base of the convective envelope of $(7-10) M_{\odot}$ stars may result in ejection of the envelope and, consequently, prevent ECSN.

Electron-capture SNe, if they really happen, presumably produce NS with short spin periods and, if in binaries, systems with short orbital periods and low eccentricities. Observational evidence for ECSN is provided by Be/X-ray binaries, which harbor two subpopulations, typical for postcore-collapse objects and for post-ECSN ones [369]. Low kicks implied for NSs formed via ECSN [597] may explain the dichotomous nature of Be/X-ray binaries. As well, thanks to low kicks, NS produced via ECSN may be more easily retained in globular clusters (e.g., [397]) or in regions of recent star formation.

The existence of the ONe-variety of WDs, predicted theoretically $[24,488,301]$ and later confirmed by observations [774], is important in the general context of compact-star binary evolution, since their accretion-induced collapse (AIC) may result in the formation of low-mass neutron stars (almost) without natal kicks and may thus be relevant to the formation of NS binaries and low-mass X-ray binaries (see $[21,146,798]$ and references therein). But since for the purpose of detection of gravitational waves they are not different from much more numerous CO WDs, we will, as a rule, 
Table 1: Types of compact remnants of single stars (the ranges of progenitor mass are shown for solar composition stars).

\begin{tabular}{ccc}
\hline Initial mass $\left[M_{\odot}\right]$ & remnant type & typical remnant mass $\left[M_{\odot}\right]$ \\
\hline $0.95<M<8-12$ & WD & 0.6 \\
$8-11<M<25-30$ & NS & 1.35 \\
$20<M<150$ & BH & $\sim 10$ \\
\hline
\end{tabular}

not consider them below as a special class.

If $M_{0} \gtrsim M_{\operatorname{mas}}$ (or 8-12 $M_{\odot}$ ), thermonuclear evolution proceeds until iron-peak elements are produced in the core. Iron cores are subject to instabilities (neutronization, nuclei photodisintegration), that lead to gravitational collapse. The core collapse of massive stars results in the formation of a neutron star or, for very massive stars, a black hole ${ }^{9}$ and is associated with the brightest astronomical phenomena such as supernova explosions (of type II, Ib, or Ib/c, according to the astronomical classification based on the spectra and light curves properties). If the precollapsing core retains significant rotation, powerful gamma-ray bursts lasting up to hundreds of seconds may be produced [850].

The boundaries between the masses of the progenitors of WDs or NSs and NSs or BHs are fairly uncertain (especially for BHs). Usually accepted typical masses of stellar remnants for single non-rotating solar-chemical-composition stars are summarized in Table 1.

Note that the above-described mass ranges for different outcomes of stellar evolution were obtained by computing 1D-models of non-rotating stars. However, all star are rotating and some of them possess magnetic fields, thus, the problem is $2 \mathrm{D}$ (or 3D, if magnetic-field effects are considered). For a physical description of effects of rotation on stellar evolution, see [406, 561]. Realistic models with rotation should account for deviation from spherical symmetry, modification of gravity due to centrifugal force, variation of radiative flux with local effective gravity, transfer of angular momentum and transport of chemical species. Up to now, as a rule, models with rotation are computed in a 1D-approximation which, typically, makes use of the fact that in rotating stars mass is constant within isobar surfaces [174]. Regretfully, for low and intermediate-mass stars, model sequences of rotating stars covering evolution to advanced phases of AGB-evolution are absent. However, as noted by Domínguez et al. [156], rotation must strongly influence evolution at core helium exhaustion and the formation of the CO-core stage, when the latter experiences very strong contraction. With an increase of angular velocity, lower pressure is necessary to balance the gravity, hence the temperature of He-burning shells of rotating stars should be lower than in nonrotating stars of the same mass. This extends AGB-lifetime and results in an increase of the mass of CO-cores, i.e., the C-ignition limit may be shifted to lower masses compared to non-rotating stars. Most recently, grids of 1-D models of rotating stars were published, e.g., in [69, 234, 236].

The algorithm and first results of self-consistent calculations of rapidly-rotating 2D stellar models of stars in the early stages of evolution are described, e.g., in [639, 178]. We may note that these models are very important for deriving physical parameters of the stars from astroseismological data.

For a more detailed introduction to the physics and evolution of stars, the reader is referred to the classical fundamental textbook [121] and to several more modern ones [612, 51, 53, 171, 297, 298]. Formation and physics of compact objects is described in more detail in the monographs [691, 53]. For recent studies and reviews of the evolution of massive stars and the mechanisms of core-

\footnotetext{
${ }^{9}$ Pair-creation instability in the oxygen cores of slowly rotating $M_{0} \approx(140-260) M_{\odot}$ and metallicity $Z \lesssim Z \odot / 3$ stars or rapidly-rotating stars with $M_{0}>80 M_{\odot}$ and $Z / Z_{\odot}<10^{-3}$ may lead to their collapse, which results in the explosive ignition of oxygen, which leads to the disruption of the star [407].
} 
collapse supernovae we refer to [406, 79, 320, 881, 212, 721].

\subsection{Binary stars}

A fundamental property of stars is their multiplicity. Among stars that complete their nuclear evolution in the Hubble time, the estimated binary fraction varies from $\sim(40-60) \%$ for $M \sim M_{\odot}$ stars $[168,621]$ to almost 100\% for more massive A/B and O-stars, e.g., [468, 382, 370, 469, 106, 671], (but, e.g., in [454, 670], a substantially lower binary fraction for massive stars is claimed).

Based on the summary of data on binary fraction $\mathcal{B}(M)$ provided in [381, 385, 671], van Haaften et al. [804] suggested an approximate formula

$$
\mathcal{B}(M) \approx \frac{1}{2}+\frac{1}{4} \log (M) \quad\left(0.08 \leq M / M_{\odot} \leq 100\right)
$$

considering all multiple systems as binaries.

The most crucial parameters of binaries include the component separation $a$ and mass ratio $q$, since for close binaries they define the outcome of the Roche lobe overflow (in fact, the fate of the system). The most recent estimates for M-dwarfs and solar-type stars confirm earlier findings that the $q$-distribution does not strongly deviate from a flat one: $d N / d q \propto q^{\beta}$, with $\beta=0.25 \pm 0.29$ [632]. This distribution is defined by the star-formation process and dynamical evolution in stellar clusters; see, e.g., [28, 569]. Distribution over $a$ is flat in $\log$ between contact and $\simeq 10^{6} R_{\odot}[604]$.

We note, cautionarily, that all estimates of the binary fraction, mass-ratios of components and distributions over separations of components are plagued by numerous selection effects (see [380] for a thorough simulation of observations and modeling observational bias). A detailed summary of studies of multiplicity rates, distributions over orbital periods and mass ratios of components for different groups of stars may be found in [167].

In binary stars with sufficiently-large orbital separations ("wide binaries") the presence of the secondary component does not influence significantly the evolution of the components. In "close binaries" the evolutionary expansion of stars leads to the overflow of the critical (Roche) lobe and mass exchange between the components RLOF. Consequently, the formation of compact remnants in close binaries differs from single stars (see Section 3 for more details).

As discussed above, the lower mass limit of NS progenitors is uncertain by several $M_{\odot}$. This limit is even more uncertain for the $\mathrm{BH}$ progenitors. For example, the presence of a magnetar (neutron star with an extremely large magnetic field) in the open cluster Westerlund 1 means that it descends from a star that is more massive than the currently-observed most-massive mainsequence cluster stars (because for a massive star the duration of the main-sequence stage is inversely proportional to its mass squared). The most massive main-sequence stars in this stellar cluster are found to have masses as high as $40 M_{\odot}$, suggesting $M_{\text {pre-BH }} \gtrsim 40 M_{\odot}$ [643]. On the other hand, it was speculated, based on the properties of X-ray sources, that in the initial mass range $(20-50) M_{\odot}$ the mass of pre-BHs may vary depending on such poorly-known parameters as rotation or magnetic fields [176].

Binaries with compact remnants are primary potentially-detectable GW sources (see Figure 2). This figure plots the sensitivity of ground-based interferometers, LIGO, as well as the space laser interferometers LISA and eLISA, in the terms of dimensionless GW strain $h$ measured over one year. ${ }^{10}$ The strongest Galactic sources at all frequencies are the most compact NS binaries, WD binaries, and (still hypothetically) BHs. NS/BH binary systems are formed from initially massive binaries, while WD binaries descend from low-mass binaries.

10 To compare the detector's sensitivity, characterized by the noise power spectral density $\sqrt{S(f)}$ with the dimensionality $1 \sqrt{\mathrm{Hz}}$, with the dimensionless GW strain amplitude $h$, one conventionally considers the detector's sensitivity in an integration time of $T$ years for a monochromatic signal, which is $\sqrt{S(f) / T}$ and corresponds to the detection at the signal-to-noise level equal one. 


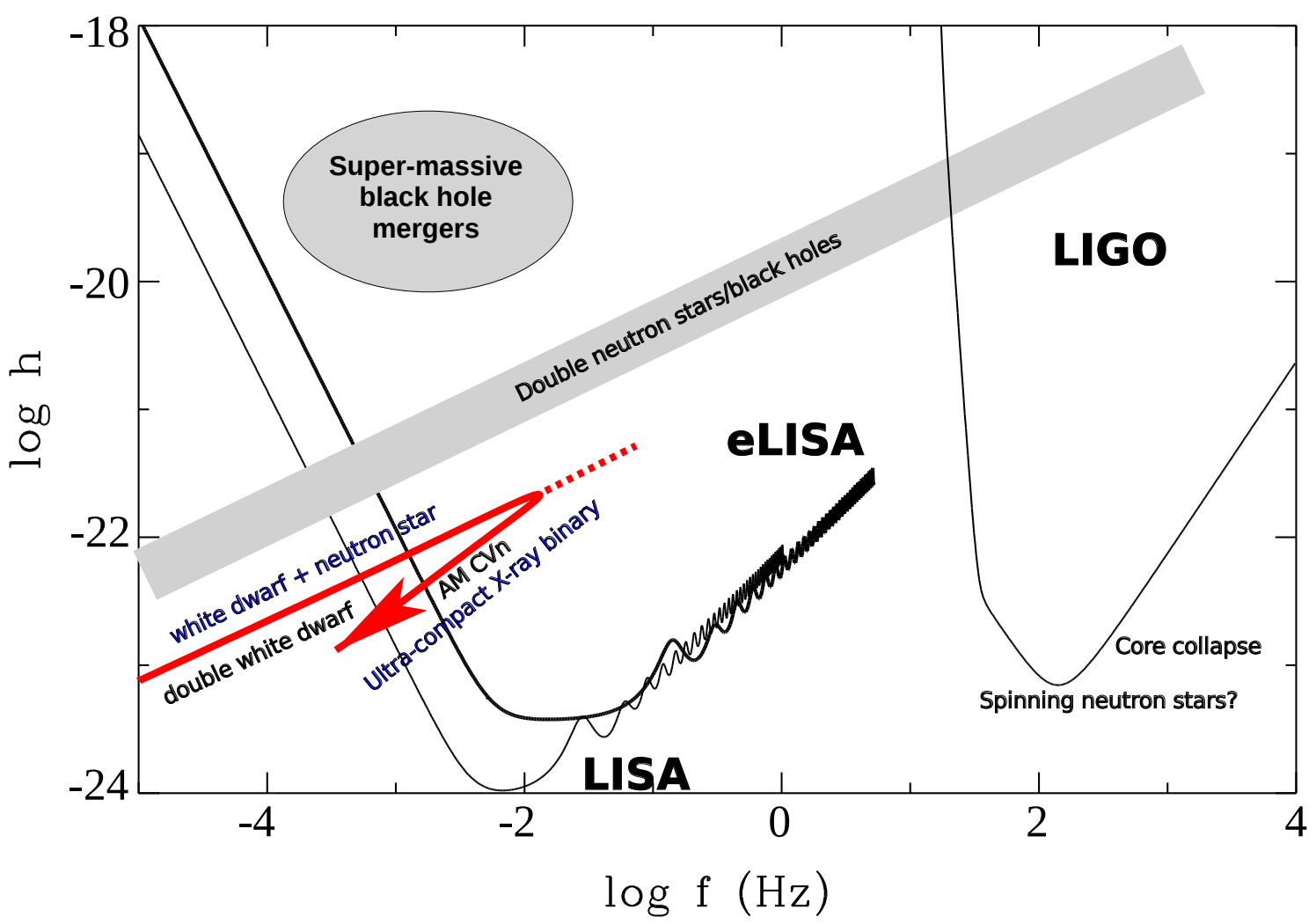

Figure 2: Sensitivity limits of GW detectors and the regions of the $f-h$ diagram occupied by some potential GW sources. (Courtesy G. Nelemans).

In this review we shall concentrate on the formation and evolution of compact star binaries most relevant to GW studies. The article is organized as follows. We start in Section 2 with a review of the main observational data on NS binaries, especially measurements of masses of NSs and BHs, which are most important for the estimate of the amplitude of the expected GW signal. We briefly discuss empirical methods to determine the NS binary coalescence rate. The basic principles of stellar binary evolution are discussed in Section 3. Then, in Section 4 we describe the evolution of massive stellar binaries. Next, we discuss the Galactic rate of formation of binaries with NSs and BHs in Section 5. Theoretical estimates of detection rates for mergers of relativistic stellar binaries are discussed in Section 6. Further, we proceed to the analysis of the formation of short-period binaries with WD components in Section 7, and consider observational data on WD binaries in Section 8. A model for the evolution of interacting double-degenerate systems is presented in Section 9. In Section 10 we describe gravitational waves from compact binaries with white dwarf components. Section 11 is devoted to the modeling of optical and X-ray emissions of AM CVn-stars. Our conclusions follow in Section 12. 


\section{Observations of Double Compact Stars}

\subsection{Compact binaries with neutron stars}

NS binaries have been discovered when one of their components is observed as a radio pulsar [292]. The precise pulsar timing allows one to search for a periodic variation due to the binary motion. This technique is reviewed in detail by Lorimer [444]; applications of pulsar timing for general relativity tests are reviewed by Stairs [733]. Techniques and results of measurements of NS mass and radii in different types of binaries are summarized by Lattimer [411].

Basically, pulsar timing provides the following Keplerian orbital parameters of the binary system: the binary orbital period $P_{\mathrm{b}}$ as measured from periodic Doppler variations of the pulsar spin, the projected semi-major axis $x=a \sin i$ as measured from the semi-amplitude of the pulsar radial velocity curve ( $i$ is the binary inclination angle defined such that $i=0$ for face-on systems), the orbital eccentricity $e$ as measured from the shape of the pulsar radial velocity curve, and the longitude of periastron $\omega$ at a particular epoch $T_{0}$. The first two parameters allow one to construct the mass function of the secondary companion,

$$
f\left(M_{\mathrm{p}}, M_{\mathrm{c}}\right)=\frac{4 \pi^{2} x^{3}}{P_{\mathrm{b}}^{2} T_{\odot}}=\frac{\left(M_{\mathrm{c}} \sin i\right)^{3}}{\left(M_{\mathrm{c}}+M_{\mathrm{p}}\right)^{2}} .
$$

In this expression, $x$ is measured in light-seconds, $T_{\odot} \equiv G M_{\odot} / c^{3}=4.925490947 \mu \mathrm{s}$, and $M_{\mathrm{p}}$ and $M_{\mathrm{c}}$ denote masses of the pulsar and its companion, respectively. This function gives the strict lower limit on the mass of the unseen companion. However, assuming the pulsar mass to have the typical value of a NS mass (for example, confined between the lowest measured NS mass $1.25 M_{\odot}$ for PSR J0737-3039B [451] and the maximum measured NS mass of $2.1 M_{\odot}$ in the NS-WD binary PSR J0751+1807 [527]), one can estimate the mass of the secondary star even without knowing the binary inclination angle $i$.

Long-term pulsar timing allows measurements of several relativistic phenomena: the advance of periastron $\dot{\omega}$, the redshift parameter $\gamma$, the Shapiro delay within the binary system qualified through post-Keplerian parameters $r, s$, and the binary orbit decay $\dot{P}_{\mathrm{b}}$. From the post-Keplerian parameters the individual masses $M_{\mathrm{p}}, M_{\mathrm{c}}$ and the binary inclination angle $i$ can be calculated [74].

Of the post-Keplerian parameters of pulsar binaries, the periastron advance rate is usually measured most readily. Assuming it to be entirely due to general relativity, the total mass of the system can be evaluated:

$$
\dot{\omega}=3\left(\frac{2 \pi}{P_{\mathrm{b}}}\right)^{5 / 3} \frac{T_{\odot}^{2 / 3}\left(M_{\mathrm{c}}+M_{\mathrm{p}}\right)^{2 / 3}}{\left(1-e^{2}\right)} .
$$

The high value of the derived total mass of a system $\left(\gtrsim 2.5 M_{\odot}\right)$ suggests the presence of another NS or even a BH. ${ }^{11}$

If the masses of components, binary period, and eccentricity of a compact binary system are known, it is easy to calculate the time it takes for the binary companions to coalesce due to GW emission using the quadrupole formula for GW emission [579] (see Section 3.1.4 for more detail):

$$
\tau_{\mathrm{GW}} \approx 4.8 \times 10^{10} \mathrm{yr}\left(\frac{P_{\mathrm{b}}}{\mathrm{d}}\right)^{8 / 3}\left(\frac{\mu}{M_{\odot}}\right)^{-1}\left(\frac{M_{\mathrm{c}}+M_{\mathrm{p}}}{M_{\odot}}\right)^{-2 / 3}\left(1-e^{2}\right)^{7 / 2} .
$$

Here $\mu=M_{\mathrm{p}} M_{\mathrm{c}} /\left(M_{\mathrm{p}}+M_{\mathrm{c}}\right)$ is the reduced mass of the binary. Some observed and derived parameters of known compact binaries with NSs are collected in Tables 2 and 3.

11 Unless the companion is directly observable and its mass can be estimated by other means. 
Table 2: Observed parameters of neutron star binaries.

\begin{tabular}{lccccccc}
\hline PSR & $\begin{array}{c}P \\
{[\mathrm{~ms}]}\end{array}$ & $\begin{array}{c}P_{\mathrm{b}} \\
{[\mathrm{d}]}\end{array}$ & $\begin{array}{c}a_{1} \sin i \\
{[\mathrm{lt}-\mathrm{s}]}\end{array}$ & $e$ & $\begin{array}{c}\dot{\omega} \\
{\left[\mathrm{deg} \mathrm{yr}^{-1}\right]}\end{array}$ & $\begin{array}{c}\dot{P}_{\mathrm{b}} \\
{\left[\times 10^{-12}\right]}\end{array}$ & Ref. \\
\hline J0737-3039A & 22.70 & 0.102 & 1.42 & 0.088 & 16.88 & -1.24 & {$[77]$} \\
J0737-3039B & 2773 & - & - & - & - & - & {$[451]$} \\
J1518+4904 & 40.93 & 8.634 & 20.04 & 0.249 & 0.011 & $?$ & {$[526]$} \\
B1534+12 & 37.90 & 0.421 & 3.73 & 0.274 & 1.756 & -0.138 & {$[844,735]$} \\
J1756-2251 & 28.46 & 0.320 & 2.75 & 0.181 & 2.585 & $?$ & {$[193]$} \\
J1811-1736 & 104.18 & 18.779 & 34.78 & 0.828 & 0.009 & $<30$ & {$[450]$} \\
J1829+2456 & 41.00 & 1.176 & 7.236 & 0.139 & 0.28 & $?$ & {$[96]$} \\
J1906+0746 & 144.07 & 0.116 & 1.42 & 0.085 & 7.57 & $?$ & {$[445]$} \\
B1913+16 & 59.03 & 0.323 & 2.34 & 0.617 & 4.227 & -2.428 & {$[292]$} \\
B2127+11C & 30.53 & 0.335 & 2.52 & 0.681 & 4.457 & -3.937 & {$[12,615]$} \\
\hline
\end{tabular}

Table 3: Derived parameters of neutron star binaries.

\begin{tabular}{lcccc}
\hline PSR & $f(m)$ & $M_{\mathrm{c}}+M_{\mathrm{p}}$ & $\tau_{c}=P /(2 \dot{P})$ & $\begin{array}{c}\tau_{\mathrm{GW}} \\
{[\mathrm{Myr}]}\end{array}$ \\
\hline J0737-3039A & 0.29 & 2.58 & 210 & 87 \\
J0737-3039B & - & - & 50 & - \\
$\mathrm{J} 1518+4904$ & 0.12 & 2.62 & & $9.6 \times 10^{6}$ \\
B1534+12 & 0.31 & 2.75 & 248 & 2690 \\
$\mathrm{~J} 1756-2251$ & 0.22 & 2.57 & 444 & 1690 \\
$\mathrm{~J} 1811-1736$ & 0.13 & 2.6 & & $1.7 \times 10^{6}$ \\
$\mathrm{~J} 1829+2456$ & 0.29 & 2.53 & & $60 \times 10^{3}$ \\
$\mathrm{~J} 1906+0746$ & 0.11 & 2.61 & 0.112 & 300 \\
$\mathrm{~B} 1913+16$ & 0.13 & 2.83 & 108 & 310 \\
$\mathrm{~B} 2127+11 \mathrm{C}$ & 0.15 & 2.71 & 969 & 220 \\
\hline
\end{tabular}




\subsection{How frequent are NS binary coalescences?}

As is seen in Table 3, only six NS binary systems presently known will merge over a time interval shorter than $\approx 10$ Gyr: J0737-3039A, B1534+12, J1756-2251, J1906+0746, B1913+16, and B2127+11C. Of these six systems, one (PSR B2127+11C) is located in the globular cluster M15. This system may have a different formation history, so usually it is not included in the analysis of the coalescence rate of Galactic compact binaries. The formation and evolution of relativistic binaries in dense stellar systems is reviewed elsewhere [39]. For a general review of pulsars in globular clusters see also [82].

Let us try to estimate the NS-binary merger rate from pulsar binary statistics, which is free from many uncertainties of stellar evolution. Usually, the estimate is based on the following extrapolation [505, 586]. Suppose, we observe $i$ classes of Galactic pulsar binaries. Taking into account various selection effects of pulsar surveys (see, e.g., [504, 361]), the Galactic number of pulsars $N_{i}$ in each class can be evaluated. To compute the Galactic merger rate of NS binaries, we need to know the time since the birth of the NS observed as a pulsar in the given binary system. This time is the sum of the observed characteristic pulsar age $\tau_{\mathrm{c}}$ and the time required for the binary system to merge due to GW orbit decay $\tau_{\mathrm{GW}}$. With the exception of PSR J0737-3039B and the recently discovered PSR J1906+0746, pulsars that we observe in NS binary systems are old recycled pulsars that were spun-up by accretion from the secondary companion to the period of several tens of ms (see Table 2). Thus, their characteristic ages can be estimated as the time since termination of spin-up by accretion (for the younger pulsar PSR J0737-3039B this time can also be computed as the dynamical age of the pulsar, $P /(2 \dot{P})$, which gives essentially the same result).

Then the merger rate $\mathcal{R}_{i}$ can be calculated as $\mathcal{R}_{i} \sim N_{i} /\left(\tau_{\mathrm{c}}+\tau_{\mathrm{GW}}\right.$ ) (summed over all pulsar binaries). The detailed analysis [361] indicates that the Galactic merger rate of NS binaries is mostly determined by pulsars with faint radio luminosity and short orbital periods. Presently, it is the nearby (600 pc) pulsar-binary system PSR J0737-3039 with a short orbital period of $2.4 \mathrm{hr}$ [77] that mostly determines the empirical estimate of the merger rate. According to Kim et al. [362], "the most likely values of DNS merger rate lie in the range 3-190 per Myr depending on different pulsar models". This estimate has recently been revised in [363] based on the analysis of binary pulsars in the Galactic disk: PSR 1913+16, PSR 1534+12, and pulsar binary PSR J0737-3039A and J0737-3039B, giving the Galactic NS + NS coalescence rate $\mathcal{R}_{G}=21_{-14-17}^{+28+40}$ per Myr $(95 \%$ and $90 \%$ confidence level, respectively). The estimates by population synthesis codes are still plagued by uncertainties in the statistics of binaries, in modeling binary evolution and supernovae. The most optimistic "theoretical" predictions amount to $\simeq 300 \mathrm{Myr}^{-1}[788,34,157]$.

An independent estimate of the NS-binary merger rate can also be obtained using another astrophysical argument, originally suggested by Bailes [20]. 1) Take the formation rate of single pulsars in the Galaxy $\mathcal{R}_{\mathrm{PSR}} \sim 1 / 50 \mathrm{yr}^{-1}$ (e.g., [190]; see also the discussion on the NS formation rate of different types in [351]), which appears to be correct to within a factor of two. 2) Take the fraction of NS binary systems in which one component is a normal (not recycled) pulsar and which are close enough to merge within the Hubble time, $f_{\mathrm{DNS}} \sim($ a few $\left.) \times 1 / 2000\right)$. Assuming a steady state, this fraction yields the formation rate of coalescing NS binaries in the Galaxy $\mathcal{R} \sim \mathcal{R}_{\mathrm{PSR}} f_{\mathrm{DNS}} \sim($ a few $10 \mathrm{~s}) \times \mathrm{Myr}^{-1}$, in good correspondence with other empirical estimates [363] Clearly, the Bailes limit ignores the fact that the NS formation rate can actually be higher than the pulsar formation rate [351] and possible selection effects related to the evolution of stars in binary systems, but the agreement with other estimates seems to be encouraging.

Extrapolation beyond the Galaxy is usually done by scaling the Galactic merger rate to the volume over which the merger events can be detected for a given GW detector's sensitivity. The scaling factor widely used is the ratio between the B-band luminosity density in the local Universe, correlating with the star-formation rate (SFR), and the B-band luminosity of the galaxy [586, 
333, 376]. For this purpose one can also use the direct ratio of the galactic star formation rate $\mathrm{SFR}_{\mathrm{G}} \simeq 2 M_{\odot} \mathrm{yr}^{-1}[108,353,537]$ to the (dust-corrected) star formation rate in the local Universe $\mathrm{SFR}_{\mathrm{loc}} \simeq 0.03 M_{\odot} \mathrm{yr}^{-1} \mathrm{Mpc}^{-3}[577,682]$. These estimates yield the (Euclidean) relation

$$
\mathcal{R}_{\mathrm{V}} \simeq 0.01 \mathcal{R}_{\mathrm{G}}\left[\mathrm{Mpc}^{-3}\right] .
$$

This estimate is very close to the local density of equivalent Milky-Way type galaxies found in [376]: 0.016 per cubic Mpc.

Bear in mind that since NS-binary coalescences can be strongly delayed compared to star formation, the estimate of their rate in the nearby Universe based on present-day star formation density may be underevaluated. Additionally, these estimates inevitably suffer from many other astrophysical uncertainties. For example, a careful study of local SFR from analysis of an almost complete sample of nearby galaxies within $11 \mathrm{Mpc}$ using different SFR indicators and supernova rate measurements during 13 years of observations [63] results in the local SFR density $\mathrm{SFR}_{\text {loc }} \simeq$ $0.008 M_{\odot} \mathrm{yr}^{-1} \mathrm{Mpc}^{-3}$. However, Horiuchi et al. [288] argue that estimates of the local SFR can be strongly affected by stellar rotation. Using new stellar evolutionary tracks [173], they derived the local SFR density $\mathrm{SFR}_{\mathrm{loc}} \simeq 0.017 M_{\odot} \mathrm{yr}^{-1} \mathrm{Mpc}^{-3}$ and noted that the estimate of the local SFR from core-collapse supernova counts is higher by a factor of $2-3$.

Therefore, the actual value of the scaling factor from the galactic merger rate is presently uncertain to within a factor of at least two. The mean SFR density steeply increases with distance (e.g., as $\left.\sim(1+z)^{3.4 \pm 0.4}[127]\right)$ and can be higher in individual galaxies. Thus, for the conservative galactic NS + NS merger rate $\mathcal{R}_{\mathrm{G}} \sim 10^{-5} \mathrm{yr}^{-1}$ using scaling relation (5) we obtain a few NSbinary coalescences per year of observations within the assumed advanced GW detectors horizon $D_{\text {hor }}=400 \mathrm{Mpc}$.

However, one should differentiate between the possible merging rate within some volume and the detection rate of certain types of compact binaries from this volume (see Section 6 below and [2] for more detail) - the detection rate by one detector can be lower by a factor of $(2.26)^{3}$. The correction factor takes into account the averaging over all sky locations and orientations.

The latest results of the search for GWs from coalescing binary systems within $40 \mathrm{Mpc}$ volume using LIGO and Virgo observations between July 7, 2009, and October 20, 2010 [3] established an observational upper limit to the $1.35 M_{\odot}+1.35 M_{\odot}$ NS binary coalescence rate of $<1.3 \times$ $10^{-4} \mathrm{yr}^{-1} \mathrm{Mpc}^{-3}$. Adopting the scaling factor from the measured local SFR density [63], the corresponding galactic upper limit is $\mathcal{R}_{G}<0.05 \mathrm{yr}^{-1}$. This is still too high to put interesting astrophysical bounds, but even upper limits from the advanced LIGO detector are expected to be very constraining.

\subsection{Black holes in binary systems}

Black holes (BH) in binary systems remain on the top of astrophysical studies. Most of the experimental knowledge on $\mathrm{BH}$ physics has so far been obtained through electromagnetic channels (see [618] for a review), but the fundamental features of BHs will be studied through GW experiments [673]. Stellar-mass black holes result from gravitational collapse of the cores of the most massive stars [851] (see [216] for the recent progress in the physics of gravitational collapse).

Stellar-mass BHs can be observed in close binary systems at the stage of mass accretion from the secondary companion as bright X-ray sources [690]. X-ray studies of BHs in binary systems (which are usually referred to as 'Black Hole Candidates', BHC) are reviewed in [633]. There is strong observational evidence of the launch of relativistic jets from the inner parts of accretion disks in $\mathrm{BHC}$, which can be observed in the range from radio to gamma-rays (the 'galactic microquasar' phenomenon, [486, 197, 756]).

Optical spectroscopy and X-ray observations of BHC allow measurements of masses and, under certain assumptions, spins of BHs [476]. The measured mass distribution of stellar mass BHs is 
centered on $\sim 8 M_{\odot}$ and appears to be separated from NS masses [550] by a gap (the absence of compact star masses in the $\sim 2-5 M_{\odot}$ range) $[22,100,549,189,580]$ (see, however, the discussion of possible systematic errors leading to overestimation of dynamical BH masses in X-ray transients in [386]). The mass gap, if real, can be indicative of the supernova-explosion-mechanism details (see, for example, [610, 37, 213]). It is also possible that the gap is due to "failed core-collapse supernovae" from red supergiants with masses $16.5 M_{\odot}<M<25 M_{\odot}$, which would produce black holes with a mass equal to the mass of the helium core $\left(5-8 M_{\odot}\right)$ before the collapse, as suggested by Kochanek [371]. Adopting the latter hypothesis would increase by $\sim 20 \%$ the stellar-mass BH formation rate. However, it is unclear how binary interaction can change the latter possibility. We stress here once again that this important issue remains highly uncertain due to the lack of "first-principles" calculations of stellar-core collapses.

Most of more than 20 galactic BHC appear as X-ray transients with a rich phenomenology of outbursts and spectral/time variability [633], and only a few (Cyg X-1, LMC X-3, LMC X-1 and SS 433) are persistent X-ray sources. Optical companions in BH transients are low-mass stars (main-sequence or evolved) filling Roche lobes, and the transient activity is apparently related to accretion-disk instability [409]. Their evolution is driven by orbital angular momentum loss due to GW emission and magnetic stellar wind [619]. Persistent BHC, in contrast, have early-type massive optical companions and belong to the class of High-Mass X-ray Binaries (HMXB) (see [755, 797] and references therein).

Like in the case of pulsar timing for NSs, to estimate the mass of the BH companion in a close binary system one should measure the radial velocity curve of the companion (usually optical) star from spectroscopic observations. The radial velocity curve (i.e., the dependence of the radial velocity of the companion on the binary phase) has a form that depends on the orbital eccentricity $e$, and the amplitude $K$. In the Newtonian limit for two point masses, the binary mass function can be expressed through the observed quantities $P_{b}$ (the binary orbital period), orbital eccentricity $e$ and semi-amplitude of the radial velocity curve $K$ as

$$
f\left(M_{v}\right)=\frac{M_{x} \sin ^{3} i}{\left(M_{x}+M_{v}\right)^{2}}=\frac{P_{b}}{2 \pi G} K_{v}^{3}\left(1-e^{2}\right)^{3 / 2} \approx 1.038 \times 10^{-7}\left[M_{\odot}\right]\left(\frac{K_{v}}{\mathrm{~km} / \mathrm{s}}\right)^{3}\left(\frac{P_{b}}{1 \mathrm{~d}}\right)\left(1-e^{2}\right)^{3 / 2} .
$$

From here one readily finds the mass of the unseen (X-ray) companion to be

$$
M_{x}=f\left(M_{v}\right)\left(1+\frac{M_{v}}{M_{x}}\right)^{2} \frac{1}{\sin ^{3} i} .
$$

Unless the mass ratio $M_{v} / M_{x}$ and orbital inclination angle $i$ are known from independent measurements (for example, from the analysis of optical light curve and the duration of X-ray eclipse), the mass function of the optical component gives the lower limit of the BH mass: $M_{x} \geq f\left(M_{v}\right)$.

When using the optical mass function to estimate the BH mass as described above, one should always check the validity of approximations used in deriving Eq. (6) (see a detailed discussion of different effects related to the non-point-like shape of the optical companion in, e.g., [99]). For example, the optical O-B or Wolf-Rayet (WR) stars in HMXBs have a powerful high-velocity stellar wind, which can affect the dynamical BH-mass estimate based on spectroscopic measurements (see the discussion in [452] for $\mathrm{BH}+\mathrm{WR}$ binaries).

Parameters of known Galactic and extragalactic HMXB with black holes are summarized in Table 4.

No PSR + BH system has been observed so far, despite optimistic expectations from the early population synthesis calculations [431] and recent examination of the possible dynamical formation of such a binary in the Galactic center [191]. Therefore, it is not possible to obtain direct experimental constraints on the $\mathrm{NS}+\mathrm{BH}$ coalescence rate based on observations of real systems. Recently, the BH candidate with Be-star MWC 656 in a wide 60-day orbit was reported [93] (see 
Table 4: Observed parameters of HMXB with black holes.

\begin{tabular}{|c|c|c|c|c|c|c|}
\hline Source & $P_{\mathrm{b}}(\mathrm{hr})$ & $f\left(M_{v}\right)\left[M_{\odot}\right]$ & $M_{x}\left[M_{\odot}\right]$ & $M_{v}\left[M_{\odot}\right]$ & Sp. type & Ref. \\
\hline \multicolumn{7}{|c|}{ Galactic binaries } \\
\hline Cyg X-1 & 134.4 & 0.244 & $\sim 15$ & $\sim 19$ & O9.7Iab & [543] \\
\hline SS 433 & 313.9 & 0.268 & $\sim 5$ & $\sim 18$ & A7I: & [101] \\
\hline Cyg X-3 & 4.8 & 0.027 & $\sim 2.4$ & $\sim 10$ & WN & [883] \\
\hline MWC 656 & 1448.9 & 0.205 & $3.8-6.9$ & $10-16$ & B1.5-B2 IIIe & {$[93]$} \\
\hline \multicolumn{7}{|c|}{ Extragalactic binaries } \\
\hline LMC X-1 & 93.8 & 0.13 & $\sim 11$ & $\sim 32$ & O7III & [545] \\
\hline M33 X-7 & 82.8 & 0.46 & $\sim 16$ & $\sim 70$ & O7-8III & [544] \\
\hline IC10 X-1 & 34.4 & 7.64 & $\sim 23-33$ & $\sim 17-35$ & WR & {$[611,710]$} \\
\hline NGC $300 \mathrm{X}-1$ & 32.3 & 2.6 & $\sim 14.5$ & $\sim 15$ & WR & {$[124]$} \\
\hline \multicolumn{7}{|c|}{ Extragalactic $\mathrm{BH}+\mathrm{WR}$ candidates } \\
\hline $\begin{array}{l}\text { CXOU J123030.3+413853 } \\
\text { (in NGC 4490) }\end{array}$ & 6.4 & $?$ & $\gtrsim 14.5$ & $?$ & WR & {$[179]$} \\
\hline $\begin{array}{l}\text { CXOU J004732.0-251722.1 } \\
\text { (in NGC 253) }\end{array}$ & $14-15$ & $?$ & $?$ & $?$ & WR & {$[452]$} \\
\hline
\end{tabular}

Table 4). It is a very weak X-ray source [498], but can be the counterpart of the gamma-ray source AGL J2241+4454. The BH mass estimation in this case depends on the spectral classification and mass of the Be star. This system can be the progenitor of the long-sought BH + NS binary. Whether or not this binary system can become a merging BH + NS binary depends on the details of the common envelope phase, which is thought to happen after the bright accretion stage in this system, when the Be-star will evolve to the giant stage.

\subsection{A model-independent upper limit on the $\mathrm{BH}-\mathrm{BH} / \mathrm{BH}-\mathrm{NS}$ coales- cence rate}

Even without using the population synthesis tool, one can search for $\mathrm{NS}+\mathrm{BH}$ or $\mathrm{BH}+\mathrm{BH}$ progenitors among known BH in HMXB. This program has been pursued in [75, 32], [33]. In these papers, the authors examined the future evolution of two bright HMXB IC10 X-1/NGC300 X-1 found in nearby low-metallicity galaxies. Both binaries consist of massive WR-stars (about $20 M_{\odot}$ ) and $\mathrm{BH}$ in close orbits (orbital periods about 30 hours). Masses of WR-stars seem to be high enough to produce the second $\mathrm{BH}$, so these system may be immediate progenitors of coalescing $\mathrm{BH}-\mathrm{BH}$ systems. Analysis of the evolution of the best-known Galactic HMXB Cyg X-1 [32], which can be a $\mathrm{NS}+\mathrm{BH}$ progenitor, led to the conclusion that the galactic formation rate of coalescing $\mathrm{NS}+\mathrm{BH}$ is likely to be very low, less than 1 per 100 Myrs. This estimate is rather pessimistic, even for advanced LIGO detectors. Implications of the growing class of short-period $\mathrm{BH}+\mathrm{WR}$ binaries (see Table 4) for the $(\mathrm{BH}+\mathrm{BH}) /(\mathrm{BH}+\mathrm{NS})$ merger rate are discussed in [452].

While for NS-binary systems it is possible to obtain the upper limit for the coalescence rate based on observed pulsar binary statistics (the Bailes limit, see Section 2.2 above), it is not so easy for $\mathrm{BH}+\mathrm{NS}$ or $\mathrm{BH}+\mathrm{BH}$ systems due to the (present-day) lack of their observational candidates. Still, a crude estimate can be found from the following considerations. A rough upper limit on the coalescence rate of $\mathrm{BH}+\mathrm{NS}(\mathrm{BH})$ binaries is set by the observed formation rate of high-mass 
X-ray binaries, their direct progenitors. The present-day formation rate of galactic HMXBs is about $\mathcal{R}_{\text {HMXв }} \sim N_{\text {HMXв }} / t_{\text {HMXB }} \sim 10^{-3}$ per year (here we conservatively assumed $N_{\text {HMXB }}=100$ and $t_{\mathrm{HMXB}}=10^{5} \mathrm{yr}$ ). This estimate can be made more precise considering that only very close compact binary systems can coalesce in the Hubble time, which requires a common envelope stage after the HMXB stage to occur (see Figure 7). The CE stage is likely to happen in sufficiently close binaries after the bright X-ray accretion stage. ${ }^{12}$ The analysis of observations of different $\mathrm{X}$-ray-source populations in the galaxies suggests ([243] and references therein) that only a few per cent of all BHs formed in a galaxy can pass through a bright accretion stage in HMXBs. For a galactic NS formation rate of once per several decades, a minimum $\mathrm{BH}$ progenitor mass of $20 M_{\odot}$ and the Salpeter initial mass function, this yields an estimate of a few $\times 10^{-5}$ per year. The probability of a given progenitor HMXB becoming a merging $\mathrm{NS}+\mathrm{BH}$ or $\mathrm{BH}+\mathrm{BH}$ binary is very model-dependent. For example, recent studies of a unique galactic microquasar SS $433[58,101]$ suggest the $\mathrm{BH}$ mass in such a system to be at least $\sim 5 M_{\odot}$ and the optical star mass to be above $15 M_{\odot}$. In SS 433, the optical star fills the Roche lobe and forms a supercritical accretion disk [183]. The mass transfer rate onto the compact star is estimated to be about $10^{-4} M_{\odot} \mathrm{yr}^{-1}$. According to the canonical HMXB evolution calculations, a common envelope must have been formed in such a binary on a short time scale (thousands of years) (see Section 3.5 and [319]), but the observed stability of binary-system parameters in SS433 over 30 years [132] shows that this is not the case. This example clearly illustrates the uncertainty in our understanding of HMXB evolution with BHs.

Thus, we conclude that in the most optimistic case where the $(\mathrm{NS}+\mathrm{BH}) /(\mathrm{BH}+\mathrm{BH})$ merger rate is equal to the formation rate of their HMXB progenitors, the upper limit for Galactic $\mathcal{R}_{\mathrm{NS}+\mathrm{BH}}$ is a few $\times 10^{-5} \mathrm{yr}^{-1}$. The detection or non-detection of such binaries within $\sim 1000 \mathrm{Mpc}$ distance by the advanced LIGO detectors can therefore very strongly constrain our knowledge of the evolution of HMXB systems.

\footnotetext{
${ }^{12}$ We neglect here the highly unlikely case of a direct collision between a newly born $\mathrm{BH}$ and its companion due to the possible $\mathrm{BH}$ natal kick.
} 


\section{Basic Principles of the Evolution of Binary Stars}

Beautiful early general reviews of the topic can be found, e.g., in [45, 795] and more later ones, e.g., in $[755,171,799]$. Here we restrict ourselves to recalling several facts concerning binary evolution that are most relevant to the formation and evolution of compact binaries. We will not discuss possible dynamical effects on binary evolution (like the Kozai-Lidov mechanism of eccentricity change in hierarchical triple systems, see e.g., [693]). Readers with experience in the field can skip this section.

\subsection{Keplerian binary system and radiation back reaction}

We start with some basic facts about Keplerian motion in a binary system and the simplest case of the evolution of two point masses due to gravitational radiation losses. The stars are highly condensed objects, so their treatment as point masses is usually adequate for the description of their interaction in the binary. Furthermore, Newtonian gravitation theory is sufficient for this purpose as long as the orbital velocities are small compared to the speed of light $c$. The systematic change of the orbit caused by the emission of gravitational waves will be considered in a separate paragraph below.

\subsubsection{Keplerian motion}

Let us consider two point masses $M_{1}$ and $M_{2}$ orbiting each other under the force of gravity. It is well known (see [404]) that this problem is equivalent to the problem of a single body with mass $\mu$ moving in an external gravitational potential. The value of the external potential is determined by the total mass of the system

$$
M=M_{1}+M_{2}
$$

The reduced mass $\mu$ is

$$
\mu=\frac{M_{1} M_{2}}{M}
$$

The body $\mu$ moves in an elliptic orbit with eccentricity $e$ and major semi-axis $a$. The orbital period $P$ and orbital frequency $\Omega=2 \pi / P$ are related to $M$ and $a$ by Kepler's third law

$$
\Omega^{2}=\left(\frac{2 \pi}{P}\right)^{2}=\frac{G M}{a^{3}} .
$$

This relationship is valid for any eccentricity $e$.

Individual bodies $M_{1}$ and $M_{2}$ move around the barycenter of the system in elliptic orbits with the same eccentricity $e$. The major semi-axes $a_{i}$ of the two ellipses are inversely proportional to the masses

$$
\frac{a_{1}}{a_{2}}=\frac{M_{2}}{M_{1}},
$$

and satisfy the relationship $a=a_{1}+a_{2}$. The position vectors of the bodies from the system's barycenter are $\vec{r}_{1}=M_{2} \vec{r} /\left(M_{1}+M_{2}\right)$ and $\vec{r}_{2}=-M_{1} \vec{r} /\left(M_{1}+M_{2}\right)$, where $\vec{r}=\vec{r}_{1}-\vec{r}_{2}$ is the relative position vector. Therefore, the velocities of the bodies with respect to the system's barycentre are related by

$$
-\frac{\vec{V}_{1}}{\vec{V}_{2}}=\frac{M_{2}}{M_{1}}
$$

and the relative velocity is $\vec{V}=\vec{V}_{1}-\vec{V}_{2}$. 
The total conserved energy of the binary system is

$$
E=\frac{M_{1} \vec{V}_{1}^{2}}{2}+\frac{M_{2} \vec{V}_{2}^{2}}{2}-\frac{G M_{1} M_{2}}{r}=\frac{\mu \vec{V}^{2}}{2}-\frac{G M_{1} M_{2}}{r}=-\frac{G M_{1} M_{2}}{2 a},
$$

where $r$ is the distance between the bodies. The orbital angular momentum vector is perpendicular to the orbital plane and can be written as

$$
\vec{J}_{\text {orb }}=M_{1} \vec{V}_{1} \times \vec{r}_{1}+M_{2} \vec{V}_{2} \times \vec{r}_{2}=\mu \vec{V} \times \vec{r} .
$$

The absolute value of the orbital angular momentum is

$$
\left|\vec{J}_{\text {orb }}\right|=\mu \sqrt{G M a\left(1-e^{2}\right)} \text {. }
$$

For circular binaries with $e=0$ the distance between orbiting bodies does not depend on time,

$$
r(t, e=0)=a,
$$

and is usually referred to as orbital separation. In this case, the velocities of the bodies, as well as their relative velocity, are also time-independent,

$$
V \equiv|\vec{V}|=\Omega a=\sqrt{G M / a},
$$

and the orbital angular momentum becomes

$$
\left|\vec{J}_{\text {orb }}\right|=\mu V a=\mu \Omega a^{2} .
$$

\subsubsection{Gravitational radiation from a binary}

The plane of the orbit is determined by the orbital angular momentum vector $\vec{J}_{\text {orb }}$. The line of sight is defined by a unit vector $\vec{n}$. The binary inclination angle $i$ is defined by the relation $\cos i=\left(\vec{n}, \vec{J}_{\text {orb }} / J_{\text {orb }}\right)$ such that $i=90^{\circ}$ corresponds to a system visible edge-on.

Let us start from two point masses $M_{1}$ and $M_{2}$ in a circular orbit. In the quadrupole approximation [405], the two polarization amplitudes of GWs at a distance $r$ from the source are given by

$$
\begin{aligned}
& h_{+}=\frac{G^{5 / 3}}{c^{4}} \frac{1}{r} 2\left(1+\cos ^{2} i\right)(\pi f M)^{2 / 3} \mu \cos (2 \pi f t), \\
& h_{\times}= \pm \frac{G^{5 / 3}}{c^{4}} \frac{1}{r} 4 \cos i(\pi f M)^{2 / 3} \mu \sin (2 \pi f t) .
\end{aligned}
$$

Here $f=\Omega / \pi$ is the frequency of the emitted GWs (twice the orbital frequency). Note that for a fixed distance $r$ and a given frequency $f$, the GW amplitudes are fully determined by $\mu M^{2 / 3}=$ $\mathcal{M}^{5 / 3}$, where the combination

$$
\mathcal{M} \equiv \mu^{3 / 5} M^{2 / 5}
$$

is called the "chirp mass" of the binary. After averaging over the orbital period (so that the squares of periodic functions are replaced by $1 / 2$ ) and the orientations of the binary orbital plane, one arrives at the averaged (characteristic) GW amplitude

$$
h(f, \mathcal{M}, r)=\left(\left\langle h_{+}^{2}\right\rangle+\left\langle h_{\times}^{2}\right\rangle\right)^{1 / 2}=\left(\frac{32}{5}\right)^{1 / 2} \frac{G^{5 / 3}}{c^{4}} \frac{\mathcal{M}^{5 / 3}}{r}(\pi f)^{2 / 3} .
$$




\subsubsection{Energy and angular momentum loss}

In the approximation and under the choice of coordinates that we are working with, it is sufficient to use the Landau-Lifshitz gravitational pseudo-tensor [405] when calculating the gravitational waves energy and flux. (This calculation can be justified with the help of a fully satisfactory gravitational energy-momentum tensor that can be derived in the field theory formulation of general relativity [17]). The energy $d E$ carried by a gravitational wave along its direction of propagation per area $d A$ per time $d t$ is given by

$$
\frac{d E}{d A d t} \equiv F=\frac{c^{3}}{16 \pi G}\left[\left(\frac{\partial h_{+}}{\partial t}\right)^{2}+\left(\frac{\partial h_{\times}}{\partial t}\right)^{2}\right] .
$$

The energy output $d E / d t$ from a localized source in all directions is given by the integral

$$
\frac{d E}{d t}=\int F(\theta, \phi) r^{2} d \Omega
$$

Replacing

$$
\left(\frac{\partial h_{+}}{\partial t}\right)^{2}+\left(\frac{\partial h_{\times}}{\partial t}\right)^{2}=4 \pi^{2} f^{2} h^{2}(\theta, \phi)
$$

and introducing

$$
h^{2}=\frac{1}{4 \pi} \int h^{2}(\theta, \phi) d \Omega
$$

we write Eq. (22) in the form

$$
\frac{d E}{d t}=\frac{c^{3}}{G}(\pi f)^{2} h^{2} r^{2}
$$

Specifically for a binary system in a circular orbit, one finds the energy loss from the system (sign minus) with the help of Eqs. (23) and (20):

$$
\frac{d E}{d t}=-\left(\frac{32}{5}\right) \frac{G^{7 / 3}}{c^{5}}(\mathcal{M} \pi f)^{10 / 3} .
$$

This expression is exactly the same that can be obtained directly from the quadrupole formula [405],

$$
\frac{d E}{d t}=-\frac{32}{5} \frac{G^{4}}{c^{5}} \frac{M_{1}^{2} M_{2}^{2} M}{a^{5}}
$$

rewritten using the definition of the chirp mass and Kepler's law. Since energy and angular momentum are continuously carried away by gravitational radiation, the two masses in orbit spiral towards each other, thus increasing their orbital frequency $\Omega$. The GW frequency $f=\Omega / \pi$ and the GW amplitude $h$ are also increasing functions of time. The rate of the frequency change is ${ }^{13}$

$$
\dot{f}=\left(\frac{96}{5}\right) \frac{G^{5 / 3}}{c^{5}} \pi^{8 / 3} \mathcal{M}^{5 / 3} f^{11 / 3} .
$$

In spectral representation, the flux of energy per unit area per unit frequency interval is given by the right-hand-side of the expression

$$
\frac{d E}{d A d f}=\frac{c^{3}}{G} \frac{\pi f^{2}}{2}\left(\left|\tilde{h}(f)_{+}\right|^{2}+\left|\tilde{h}(f)_{\times}\right|^{2}\right) \equiv \frac{c^{3}}{G} \frac{\pi f^{2}}{2} S_{h}^{2}(f),
$$

\footnotetext{
13 A signal with such an increasing frequency is reminiscent of the chirp of a bird. This explains the origin of the term "chirp mass" for the parameter $\mathcal{M}$, which fully determines the GW frequency and amplitude behavior.
} 
where we have introduced the spectral density $S_{h}^{2}(f)$ of the gravitational wave field $h$. In the case of a binary system, the quantity $S_{h}$ is calculable from Eqs. (18 and 19):

$$
S_{h}=\frac{G^{5 / 3}}{c^{3}} \frac{\pi}{12} \frac{\mathcal{M}^{5 / 3}}{r^{2}} \frac{1}{(\pi f)^{7 / 3}} .
$$

\subsubsection{Binary coalescence time}

A binary system in a circular orbit loses energy according to Eq. (24). For orbits with non-zero eccentricity $e$, the right-hand-side of this formula should be multiplied by the factor

$$
f(e)=\left(1+\frac{73}{24} e^{2}+\frac{37}{96} e^{4}\right)\left(1-e^{2}\right)^{-7 / 2}
$$

(see [579]). The initial binary separation $a_{0}$ decreases and, assuming Eq. (25) is always valid, the binary should vanish in a time

$$
t_{0}=\frac{c^{5}}{G^{3}} \frac{5 a_{0}^{4}}{256 M^{2} \mu}=\frac{5 c^{5}}{256} \frac{\left(P_{0} / 2 \pi\right)^{8 / 3}}{(G \mathcal{M})^{5 / 3}} \approx\left(9.8 \times 10^{6} \mathrm{yr}\right)\left(\frac{P_{0}}{1 \mathrm{~h}}\right)^{8 / 3}\left(\frac{\mathcal{M}}{M_{\odot}}\right)^{-5 / 3}
$$

As noted above, gravitational radiation from the binary depends on the chirp mass $\mathcal{M}$, which can also be written as $\mathcal{M} \equiv M \eta^{3 / 5}$, where $\eta$ is the dimensionless ratio $\eta=\mu / M$. Since $\eta \leq 1 / 4$, one has $\mathcal{M} \lesssim 0.435 M$. For example, for two NSs with equal masses $M_{1}=M_{2}=1.4 M_{\odot}$, the chirp mass is $\mathcal{M} \approx 1.22 M_{\odot}$. This explains the choice of normalization in Eq. (29).

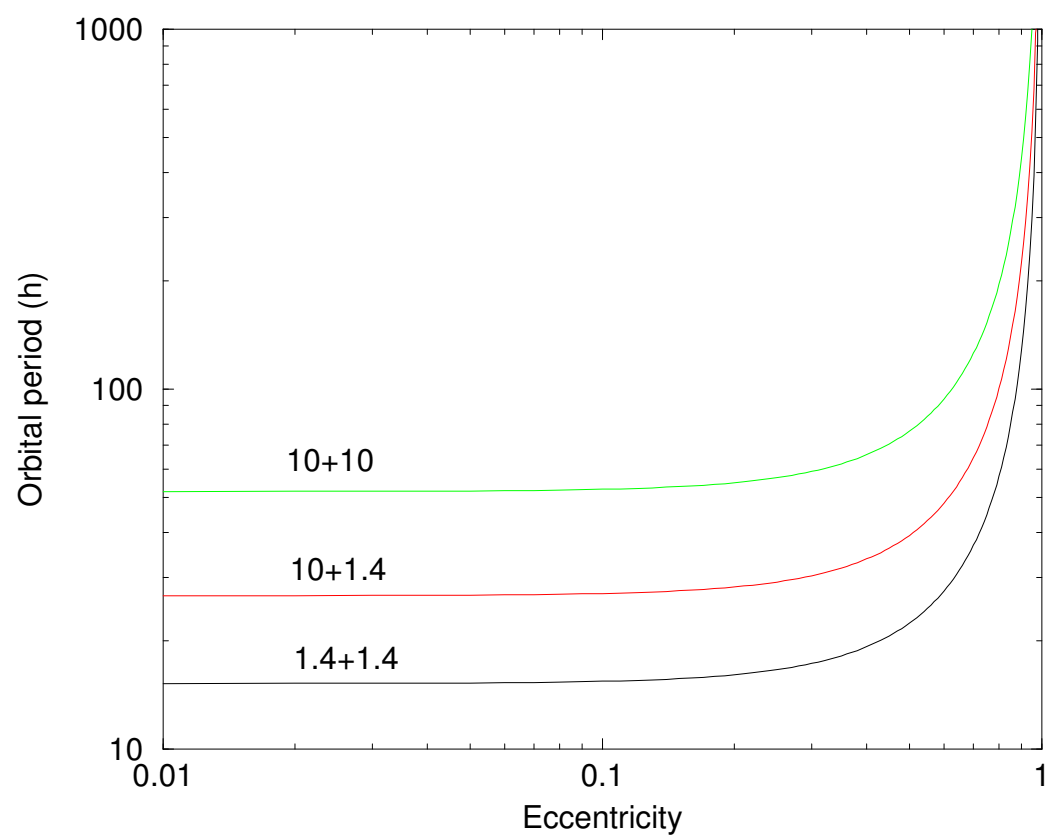

Figure 3: The maximum initial orbital period (in hours) of two point masses that will coalesce due to gravitational wave emission in a time interval shorter than $10^{10} \mathrm{yr}$, as a function of the initial eccentricity $e_{0}$. The lines are calculated for $10 M_{\odot}+10 M_{\odot}(\mathrm{BH}+\mathrm{BH}), 10 M_{\odot}+1.4 M_{\odot}(\mathrm{BH}+\mathrm{NS})$, and $1.4 M_{\odot}+1.4 M_{\odot}$ $(\mathrm{NS}+\mathrm{NS})$.

The coalescence time for a binary star with an initially eccentric orbit with $e_{0} \neq 0$ and initial separation $a_{0}$ is shorter than the coalescence time for an object with a circular orbit and the same 
$a_{0}[579]:$

$$
t_{\mathrm{c}}\left(e_{0}\right)=t_{0} f\left(e_{0}\right)
$$

where the correction factor $f\left(e_{0}\right)$ is

$$
f\left(e_{0}\right)=\frac{48}{19} \frac{\left(1-e_{0}^{2}\right)^{4}}{e_{0}^{48 / 19}\left(1+\frac{121}{304} e_{0}^{2}\right)^{3480 / 2299}} \int_{0}^{e_{0}} \frac{\left(1+\frac{121}{304} e^{2}\right)^{1181 / 2299}}{\left(1-e^{2}\right)^{3 / 2}} e^{29 / 19} d e .
$$

To merge in a time interval shorter than $10 \mathrm{Gyr}$ the binary should have a small enough initial orbital period $P_{0} \leq P_{\mathrm{cr}}\left(e_{0}, \mathcal{M}\right)$ and, accordingly, a small enough initial semi-major axis $a_{0} \leq$ $a_{\mathrm{cr}}\left(e_{0}, \mathcal{M}\right)$. The critical orbital period is plotted as a function of the initial eccentricity $e_{0}$ in Figure 3. The lines are plotted for three typical sets of masses: two neutron stars with equal masses $\left(1.4 M_{\odot}+1.4 M_{\odot}\right)$, a black hole and a neutron star $\left(10 M_{\odot}+1.4 M_{\odot}\right)$, and two black holes with equal masses $\left(10 M_{\odot}+10 M_{\odot}\right)$. Note that in order to get a significantly shorter coalescence time, the initial binary eccentricity should be $e_{0} \geq 0.6$.

\subsubsection{Magnetic stellar wind}

In the case of low-mass binary evolution, there is another important physical mechanism responsible for the removal of orbital angular momentum, in addition to the GW emission discussed above. This is the magnetic stellar wind (MSW), or magnetic braking, which is thought to be effective for main-sequence G-M dwarfs with convective envelopes, i.e., approximately, in the mass interval $0.3-1.2 M_{\odot}$. The upper mass limit corresponds to the disappearance of a deep convective zone, while the lower mass limit stands for fully convective stars. In both cases a dynamo mechanism, responsible for enhanced magnetic activity, is thought to become ineffective. The idea behind angular momentum loss (AML) by magnetically-coupled stellar wind is that the stellar wind is compelled by magnetic field to corotate with the star to rather large distances, where it carries away large specific angular momentum [680]. Thus, it appears possible to take away substantial angular momentum without evolutionarily significant mass-loss in the wind. In the quantitative form, the concept of angular momentum loss by MSW as a driver of the evolution of compact binaries was introduced by Verbunt and Zwaan [810] when it became evident that momentum loss by GWs is unable to explain the observed mass-transfer rates in cataclysmic variables (CVs) and low-mass $\mathrm{X}$ ray binaries, as well as the deficit of cataclysmic variables with orbital periods between 2 and $3 \mathrm{hr}$ (the "period gap" ). ${ }^{14}$ Verbunt and Zwaan based their reasoning on observations of the spin-down of rotation of single G-dwarfs in stellar clusters with age, which is expressed by the phenomenological dependence of the equatorial rotational velocity $V$ on age: $V=\lambda t^{-1 / 2}$ ("Skumanich law" [718]). In the latter formula $\lambda$ is an empirically-derived coefficient $\sim 1$. Applying this to a binary component and assuming tidal locking between the stellar axial rotation and orbital motion, one arrives at the rate of angular momentum loss via MSW

$$
\dot{J}_{\mathrm{MSW}}=-0.5 \times 10^{-28}\left[\mathrm{~g} \mathrm{~cm}^{2} \mathrm{~s}^{-2}\right] \lambda^{-2} k^{2} M_{2} R_{2}^{4} \omega^{3},
$$

where $M_{2}$ and $R_{2}$ are the mass and radius of the optical component of the system, respectively, $\omega=\Omega_{\text {orb }}$ is the spin frequency of the star's rotation equal to the binary orbital frequency, and $k^{2} \sim 0.1$ is the gyration radius of the optical component squared.

Radii of stars filling their Roche lobes should be proportional to binary separations, $R_{\mathrm{o}} \propto a$, which means that the time scale of orbital angular momentum removal by MSW is $\tau_{\mathrm{MSW}} \equiv$ $\left(\dot{J}_{\mathrm{MSW}} / J_{\mathrm{orb}}\right)^{-1} \propto a$. This should be compared with AML by GWs with $\tau_{\mathrm{GW}} \propto a^{4}$. Clearly, MSW (if it operates) is more efficient at removing angular momentum from a binary system at larger separations (orbital periods), and at small orbital periods GWs always dominate. Magnetic

\footnotetext{
14 Actually, no CVs were known in the "gap" when this fact was first realized [647].
} 
braking is especially important in CVs and in LMXBs with orbital periods exceeding several hours and is the driving mechanism for mass accretion onto the compact component. Indeed, the Doppler tomography reconstruction of the Roche-lobe-filling low-mass K7 secondary star in a well-studied LMXB Cen X-4 revealed the presence of cool spots on its surface. The latter provide evidence for the action of magnetic fields at the surface of the star, thus supporting magnetic braking as the driving mechanism of mass exchange in this binary [689].

Equation (32) with assumed $\lambda=1$ and $k^{2}=0.07$ [627] is often considered as a "standard". However, Eqs. (25) and (32) do not allow one to reproduce some observed features of CVs, see, e.g., $[627,600,434,771,368]$, but the reasons for this discrepant behavior are not clear as yet. Recently, Knigge et al. [368], based on the study of properties of donors in CVs, suggested that a better description of the evolution of CVs is provided by scaling Eq. (32) by a factor of $(0.66 \pm$ $0.05)\left(R_{2} / R_{\odot}\right)^{-1}$ above the period gap and by scaling Eq. (25) by a factor $2.47 \pm 0.22$ below the gap (where AML by MSW is not acting). Note that these "semi-empirical", as called by the their authors, numerical factors must be taken with some caution, since they are based on the fitting of the $P_{\mathrm{orb}}-M_{2}$ relation for observed stars by a single evolutionary sequence with the initiallyunevolved donor with $M_{2,0}=1 M_{\odot}$ and $M_{\mathrm{WD}, 0}=0.6 M_{\odot}$.

The simplest reason for deviation of AML by MSW from Eq. (25) may be the unjustified extrapolation of stellar-rotation rates over several orders of magnitude - from slowly-rotating single field stars to rapidly-spinning components of close binaries. For the shortest orbital periods of binaries meant to evolve due to AML via GW, the presence of circumbinary discs may enhance their orbital angular momentum loss [841].

\subsection{Mass exchange in close binaries}

As mentioned in the introductory Section 1, all binaries may be considered either as "close" or as "wide". In the former case, mass exchange between the components can occur. This process can be accompanied by mass and angular momentum loss from the system.

The shape of the stellar surface is determined by the shape of the equipotential level surface $\Phi=$ const. Conventionally, the total potential, which includes gravitational and centrifugal forces is approximated by the Roche potential (see, e.g., [375]), which is defined under the following assumptions:

the gravitational field of two components is approximated by that of two point masses;

the binary orbit is circular;

the components of the system corotate with the binary orbital period.

Let us consider a Cartesian reference frame $(\mathrm{x}, \mathrm{y}, \mathrm{z})$ rotating with the binary, with the origin at the primary $M_{1}$; the x-axis is directed along the line of centers; the y-axis is aligned with the orbital motion of the primary component; the z-axis is perpendicular to the orbital plane. The total potential at a given point $(\mathrm{x}, \mathrm{y}, \mathrm{z})$ is then

$$
\Phi=-\frac{G M_{1}}{\left[x^{2}+y^{2}+z^{2}\right]^{1 / 2}}-\frac{G M_{2}}{\left[(x-a)^{2}+y^{2}+z^{2}\right]^{1 / 2}}-\frac{1}{2} \Omega_{\text {orb }}^{2}\left[(x-\mu a)^{2}+y^{2}\right],
$$

where $\mu=M_{2} /\left(M_{1}+M_{2}\right), \Omega_{\text {orb }}=2 \pi / P_{\text {orb }}$.

A 3-D representation of a dimensionless Roche potential in a co-rotating frame for a binary with mass ratio of components $q=2$ is shown in Figure 4.

For close binary evolution the most important is the innermost level surface that encloses both components. It defines the "critical", or "Roche" lobes of the components. ${ }^{15}$ Inside these lobes

15 Actually, the Roche potential should also incorporate the effects of radiation pressure from the binary components. Then in some cases the resulting potentials do not exhibit the contact surfaces of the classical Roche potential [684, 144]. 


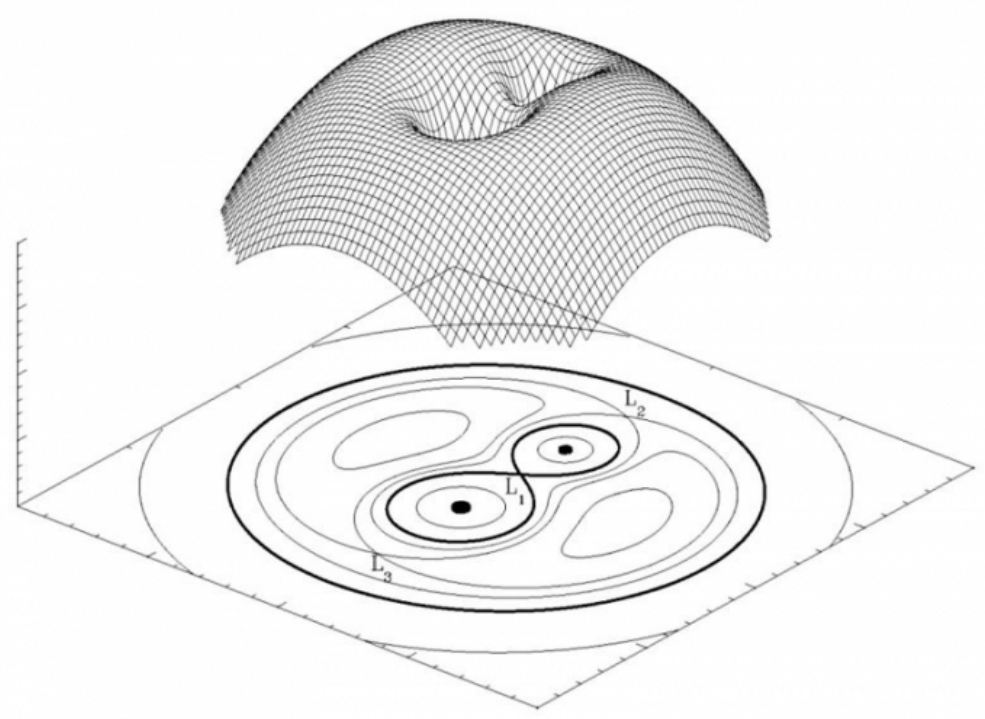

Figure 4: A 3-D representation of a dimensionless Roche potential in the co-rotating frame for a binary with a mass ratio of components $q=2$.

the matter is bound to the respective component. In the libration point $L_{1}$ (the inner Lagrangian point) the net force exerted onto a test particle corotating with the binary vanishes, so the particle can escape from the surface of the star and be captured by the companion. The matter flows along the surface of the Roche-lobe filling companion in the direction of $L_{1}$ and escapes from the surface of the contact component as a highly inhomogeneous stellar wind. Next, the wind forms a (supersonic) stream directed at a certain angle respective to the line connecting the centers of the components. Depending on the size of the second companion, the stream may hit the latter (called "direct impact") or form a disk orbiting the companion [448].

Though it is evident that mass exchange is a complex 3D gas-dynamical process that must also take into account radiation transfer and, in some cases, even nuclear reactions, virtually all computations of the evolution of non-compact close binaries have been performed in 1-D approximations. The Roche-lobe overflow (RLOF) is conventionally considered to begin when the radius of an initially more massive and hence faster evolving star (primary component) becomes equal to the radius of a sphere with volume equal to that of the Roche lobe. For the latter, an expression precise to better than $1 \%$ for an arbitrary mass ratio of components $q$ was suggested by Eggleton [172]:

$$
\frac{R_{l}}{a} \approx \frac{0.49 q^{2 / 3}}{0.6 q^{2 / 3}+\ln \left(1+q^{1 / 3}\right)} .
$$

For practical purposes, such as analytical estimates, a more convenient expression is that suggested by Kopal [375] (usually called the "Paczyński formula", who introduced it into close binary modeling):

$$
\frac{R_{l}}{a} \approx 0.4623\left(\frac{q}{1+q}\right)^{1 / 3},
$$

which is accurate to $\lesssim 2 \%$ for $0<q \lesssim 0.8$.

In close binaries, the zero-age main sequence (ZAMS) mass ceases to be the sole parameter determining stellar evolution. The nature of compact remnants of close binary components also 
depends on their evolutionary stage at RLOF, i.e., on the component separation and their massratio $q$. Evolution of a star may be considered as consumption of nuclear fuel accompanied by an increase of its radius. Following the pioneering work of Kippenhahn and his collaborators on the evolution of close binaries in the late 1960s, the following basic cases of mass exchange are usually considered: $\mathbf{A}$ - RLOF at the core hydrogen-burning stage; $\mathbf{B}$ - RLOF at the hydrogen-shell burning stage; $\mathbf{C}-$ RLOF after exhaustion of He in the stellar core. Also, more "fine" gradations exist: case $\mathbf{A B}$ - RLOF at the late stages of core H-burning, which continues as Case $\mathbf{B}$ after a short break upon exhaustion of $\mathrm{H}$ in the core; case $\mathbf{B B}-\mathrm{RLOF}$ by the star, which first filled its Roche lobe in case $\mathbf{B}$, contracted under the Roche lobe after the loss of the hydrogen envelope, and resumed the mass loss due to the envelope expansion at the helium-shell burning stage. Further, one may consider the modes of mass-exchange, depending, e.g., on the nature of the envelope of the donor (radiative vs. convective), its relative mass, reaction of accretor etc., see, e.g., [171, 826] and Section 3.3.

In cases $\mathbf{A}$ and $\mathbf{B}$ of mass exchange the remnants of stars with initial masses lower than $(2.3-2.8) M_{\odot}$ are degenerate He WD - a type of objects not produced by single stars. ${ }^{16}$

In cases $\mathbf{A}$ and $\mathbf{B}$ of mass exchange the remnants of stars with initial mass $\gtrsim 2.5 M_{\odot}$ are helium stars.

If the mass of the helium remnant star does not exceed $\simeq 0.8 M_{\odot}$, after exhaustion of the $\mathrm{He}$ in the core it does not expand, but transforms directly into a WD of the same mass [300]. Helium stars with masses between $\simeq 0.8 M_{\odot}$ and $\simeq(2.3-2.8) M_{\odot}$ expand in the helium shell burning stage, re-fill their Roche lobes, lose the remnants of the helium envelopes and also transform into CO WD.

Stellar radius may be taken as a proxy for the evolutionary state of a star. We plot in Figure 5 the types of stellar remnant as a function of both initial mass and the radius of a star at the instant of RLOF. In Figure 6 initial-final mass relations for components of close binaries are shown. Clearly, relations presented in these two figures are only approximate, reflecting current uncertainty in the theory of stellar evolution (in this particular case, relations used in the population synthesis code IBIS [791] are shown.

\subsection{Mass transfer modes and mass and angular momentum loss in binary systems}

GW emission is the sole factor responsible for the change of orbital parameters of a detached pair of compact (degenerate) stars. However, it was recognized quite early on that in the stages of evolution preceding the formation of compact objects, the mode of mass transfer between the components and the loss of matter and orbital angular momentum by the system as a whole play a dominant dynamical role and define the observed features of the binaries, e.g., [556, 781, 631, 471, 603, 137].

Strictly speaking, as mentioned previously, these processes should be treated hydrodynamically and they require complicated numerical calculations. However, binary evolution can also be described semi-qualitatively, using a simplified description in terms of point-like bodies. The change of their integrated physical quantities, such as masses, orbital angular momentum, etc., governs the evolution of the orbit. This description turns out to be successful in reproducing the results of more rigorous numerical calculations (see, e.g., [705] for more details and references). In this approach, the key role is allocated to the total orbital angular momentum $J_{\text {orb }}$ of the binary.

Let star 2 lose matter at a rate $\dot{M}_{2}<0$ and let $\beta(0 \leq \beta \leq 1)$ be the fraction of the ejected matter that leaves the system (the rest falls on the first star), i.e., $\dot{M}_{1}=-(1-\beta) \dot{M}_{2} \geq 0$. Consider circular orbits with orbital angular momentum given by Eq. (17). Differentiate both parts of Eq. (17) by time $t$ and exclude $d \Omega / d t$ with the help of Kepler's third law (10). This gives

${ }^{16}$ Hydrogen burning time for solar-composition single stars with $M_{0} \lesssim 0.95 M_{\odot}$ exceeds the age of the universe. 


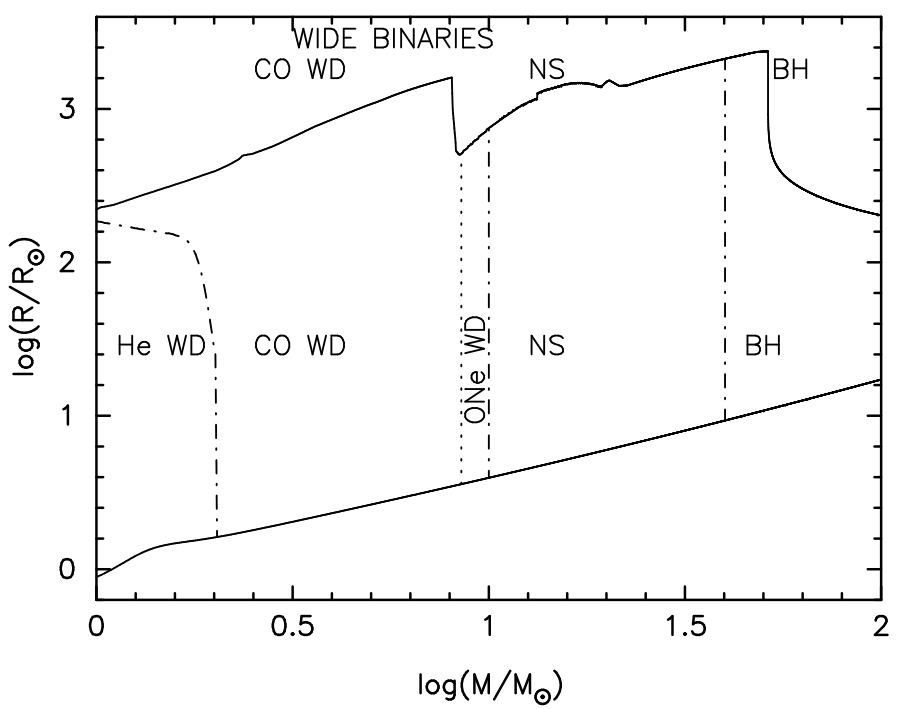

Figure 5: Descendants of components of close binaries depending on the radius of the star at RLOF. The upper solid line separates close and wide binaries (after [293]). The boundary between progenitors of Heand CO-WDs is uncertain by several $0.1 M_{\odot}$, the boundary between $\mathrm{CO}$ and ONe varieties of WDs and WD and NSs - by $\sim 2 M_{\odot}$. The boundary between progenitors of NS and BH is shown at $40 M_{\odot}$ after [643], while it may be possible that it really is between $20 M_{\odot}$ and $50 M_{\odot}$ (see Section 1 for discussion and references.)

the rate of change of the orbital separation:

$$
\frac{\dot{a}}{a}=-2\left(1+(\beta-1) \frac{M_{2}}{M_{1}}-\frac{\beta}{2} \frac{M_{2}}{M}\right) \frac{\dot{M}_{2}}{M_{2}}+2 \frac{\dot{J}_{\text {orb }}}{J_{\text {orb }}} .
$$

In Eq. (36) $\dot{a}$ and $\dot{M}$ are not independent variables if the donor fills its Roche lobe. One defines the mass transfer as conservative if both $\beta=0$ and $\dot{J}_{\text {orb }}=0$. The mass transfer is called nonconservative if at least one of these conditions is violated.

It is important to distinguish some specific cases (modes) of mass transfer:

1. conservative mass transfer,

2. non-conservative Jeans mode of mass loss (or fast wind mode),

3. non-conservative isotropic re-emission,

4. sudden mass loss from one of the components during supernova explosion, and

5. common-envelope stage.

As specific cases of angular momentum loss we consider GW emission (see Section 3.1.3 and 3.1.4) and the magnetically-coupled stellar wind (see Section 3.1.5), which drive the orbital evolution for short-period binaries. For non-conservative modes, one can also consider some less important cases, such as, for instance, the formation of a circumbinary ring by the matter leaving the system (see, e.g., [722, 732]). Here, we will not go into details of such sub-cases. 


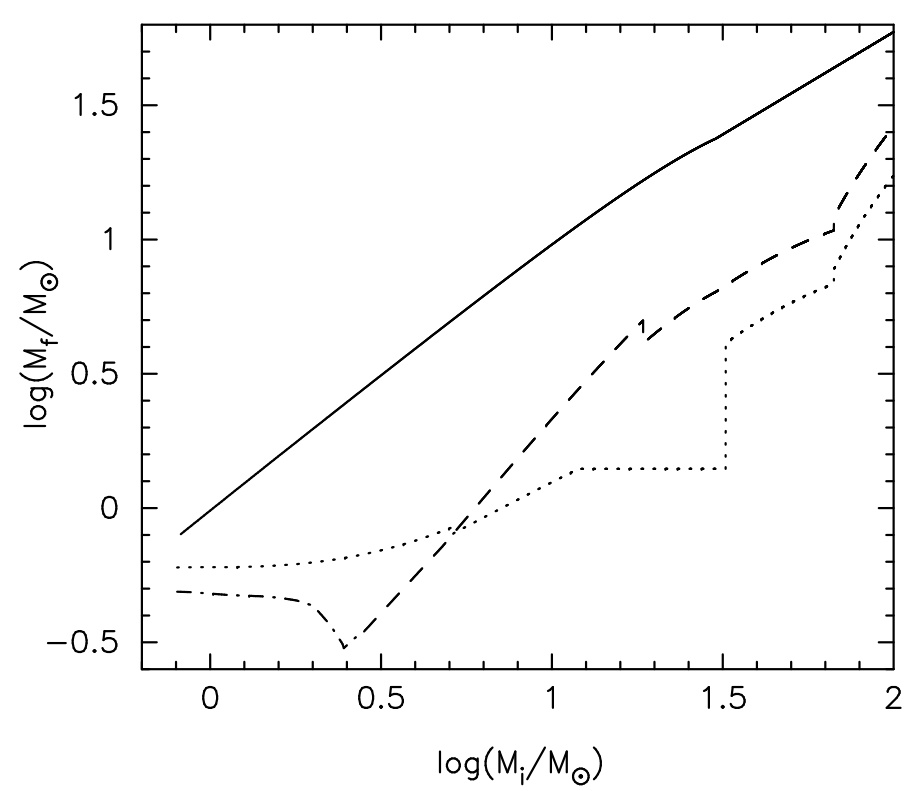

Figure 6: Relation between ZAMS masses of stars $M_{i}$ and their masses at TAMS (solid line), masses of helium stars (dashed line), masses of He WD (dash-dotted line), masses of CO and ONe WD (dotted line). For stars with $M_{i} \lesssim 5 M_{\odot}$ we plot the upper limit of WD masses for case $\mathbf{B}$ of mass exchange. After [791].

\subsubsection{Conservative accretion}

In the case of conservative accretion, matter from $M_{2}$ is fully deposited onto $M_{1}$. The transfer process preserves the total mass $(\beta=0)$ and the orbital angular momentum of the system. It follows from Eq. (36) that

$$
M_{1} M_{2} \sqrt{a}=\text { const., }
$$

so that the initial and final binary separations are related as

$$
\frac{a_{\mathrm{f}}}{a_{\mathrm{i}}}=\left(\frac{M_{1 \mathrm{i}} M_{2 \mathrm{i}}}{M_{1 \mathrm{f}} M_{2 \mathrm{f}}}\right)^{2}
$$

The orbit shrinks when the more massive component loses matter, and the orbit widens in the opposite situation. During such a mass exchange, the orbital separation passes through a minimum, if the masses become equal in the course of mass transfer.

\subsubsection{The Jeans (fast wind) mode}

In this mode the matter ejected by the donor completely escapes from the system, that is, $\beta=1$. Escape of the matter can occur either via fast isotropic spherically-symmetric wind or in the form of bipolar jets moving from the system at high velocity. Escaping matter does not interact with another component. The matter escapes with the specific angular momentum of the mass-losing star $J_{2}=\left(M_{1} / M\right) J_{\text {orb }}$ (we neglect a possible proper rotation of the star, see [795]). For the loss of orbital momentum $\dot{J}_{\text {orb }}$ it is reasonable to take

$$
\dot{J}_{\text {orb }}=\frac{\dot{M}_{2}}{M_{2}} J_{2}
$$


In the case $\beta=1$, Eq. (36) can be written as

$$
\frac{\dot{\Omega} a^{2}}{\Omega a^{2}}=\frac{\dot{J}_{\text {orb }}}{J_{\text {orb }}}-\frac{M_{1} \dot{M}_{2}}{M M_{2}} .
$$

Then Eq. (39) in conjunction with Eq. (38) gives $\Omega a^{2}=$ const, that is, $\sqrt{G a M}=$ const. Thus, as a result of the Jeans mode of mass loss, the change in orbital separation is

$$
\frac{a_{\mathrm{f}}}{a_{\mathrm{i}}}=\frac{M_{\mathrm{i}}}{M_{\mathrm{f}}} .
$$

Since the total mass decreases, the orbit always widens.

\subsubsection{Isotropic re-emission}

The matter lost by star 2 can first accrete onto star 1, and then, a fraction $\beta$ of the accreted matter, can be expelled from the system. This happens, for instance, when a massive star transfers matter onto a compact star on the thermal timescale (usually $<10^{6}$ years). Accretion luminosity may exceed the Eddington luminosity limit, and the radiation pressure pushes the infalling matter away from the system, in a manner similar to the spectacular example of the SS 433 binary system. Other examples may be systems with helium stars transferring mass onto relativistic objects [442, 238]. In this mode of mass-transfer, the binary orbital momentum carried away by the expelled matter is determined by the orbital momentum of the accreting star rather than by the orbital momentum of the mass-losing star, since mass loss happens in the vicinity of the accretor. The assumption that all matter in excess of accretion rate can be expelled from the system, thus avoiding the formation of a common envelope, will only hold if the liberated accretion energy of the matter falling from the Roche lobe radius of the accretor star to its surface is sufficient to expel the matter from the Roche-lobe surface around the accretor, i.e., $\dot{M}_{\mathrm{d}} \lesssim \dot{M}_{\mathrm{d}_{\max }}=\dot{M}_{\mathrm{Edd}}\left(r_{\mathrm{L}, \mathrm{a}} / r_{\mathrm{a}}\right)$, where $r_{\mathrm{a}}$ is the radius of the accretor [364].

The orbital momentum loss can be written as

$$
\dot{J}_{\text {orb }}=\beta \frac{\dot{M}_{2}}{M_{1}} J_{1},
$$

where $J_{1}=\left(M_{2} / M\right) J_{\text {orb }}$ is the orbital momentum of the star $M_{1}$. In the limiting case when all the mass initially accreted by $M_{1}$ is later expelled from the system, $\beta=1$, Eq. (41) simplifies to

$$
\frac{\dot{J}_{\text {orb }}}{J_{\text {orb }}}=\frac{\dot{M}_{2} M_{2}}{M_{1} M} .
$$

After substitution of this formula into Eq. (36) and integration over time, one arrives at

$$
\frac{a_{\mathrm{f}}}{a_{\mathrm{i}}}=\frac{M_{\mathrm{i}}}{M_{\mathrm{f}}}\left(\frac{M_{2 \mathrm{i}}}{M_{2 \mathrm{f}}}\right)^{2} \exp \left(-2 \frac{M_{2 \mathrm{i}}-M_{2 \mathrm{f}}}{M_{1}}\right) .
$$

The exponential term makes this mode of mass transfer very sensitive to the components' mass ratio. If $M_{1} / M_{2} \ll 1$, the separation $a$ between the stars may decrease so much that the approximation of point masses becomes invalid. Tidal orbital instability (Darwin instability) may set in, and the compact star's may start spiraling toward the companion star centre (the common envelope stage; see Section 3.6). On the other hand, "isotropic reemission" may stabilize mass-exchange if $M_{1} / M_{2}>1$ [877].

Mass loss may be considered as occurring in the "isotropic re-emission" mode in situations in which hot white dwarf components of cataclysmic variables lose mass by optically-thick winds [346] or when time-averaged mass loss from novae is considered [867]. 


\subsection{Supernova explosion}

Supernovae explosions in binary systems occur on a timescale much shorter than the orbital period, so the loss of mass is practically instantaneous. This case can be treated analytically (see, e.g., $[55,59,753])$.

Clearly, even a spherically-symmetric sudden mass loss due to an SN explosion will be asymmetric in the reference frame of the center of mass of the binary system, leading to system recoil ('Blaauw-Boersma' recoil). In general, the loss of matter and radiation is non-spherical, so that the remnant of the supernova explosion (neutron star or black hole) acquires some recoil velocity called kick velocity $\vec{w}$. In a binary, the kick velocity should be added to the orbital velocity of the pre-supernova star.

The usual treatment proceeds as follows. Let us consider a pre-SN binary with initial masses $M_{1}$ and $M_{2}$. The stars move in a circular orbit with orbital separation $a_{\mathrm{i}}$ and relative velocity $\vec{V}_{\mathrm{i}}$. The star $M_{1}$ explodes leaving a compact remnant of mass $M_{\mathrm{c}}$. The total mass of the binary decreases by $\Delta M=M_{1}-M_{\mathrm{c}}$. Unless the binary is disrupted, it will end up in a new orbit with eccentricity $e$, semi-major axis $a_{\mathrm{f}}$, and angle $\theta$ between the orbital planes before and after the explosion. In general, the new barycenter will also receive some velocity, but we neglect this motion. The goal is to evaluate the parameters $a_{\mathrm{f}}, e$, and $\theta$.

It is convenient to use an instantaneous reference frame centered on $M_{2}$ right at the time of explosion. The $x$-axis is the line from $M_{2}$ to $M_{1}$, the $y$-axis points in the direction of $\vec{V}_{\mathrm{i}}$, and the $z$-axis is perpendicular to the orbital plane. In this frame, the pre-SN relative velocity is $\vec{V}_{\mathrm{i}}=\left(0, V_{\mathrm{i}}, 0\right)$, where $V_{\mathrm{i}}=\sqrt{G\left(M_{1}+M_{2}\right) / a_{\mathrm{i}}}$ (see Eq. (16)). The initial total orbital momentum is $\overrightarrow{J_{\mathrm{i}}}=\mu_{\mathrm{i}} a_{\mathrm{i}}\left(0,0,-V_{\mathrm{i}}\right)$. The explosion is considered to be instantaneous. Right after the explosion, the position vector of the exploded star $M_{1}$ has not changed: $\vec{r}=\left(a_{\mathrm{i}}, 0,0\right)$. However, other quantities have changed: $\vec{V}_{\mathrm{f}}=\left(w_{x}, V_{\mathrm{i}}+w_{y}, w_{z}\right)$ and $\vec{J}_{\mathrm{f}}=\mu_{\mathrm{f}} a_{\mathrm{i}}\left(0, w_{z},-\left(V_{\mathrm{i}}+w_{y}\right)\right)$, where $\vec{w}=\left(w_{x}, w_{y}, w_{z}\right)$ is the kick velocity and $\mu_{\mathrm{f}}=M_{\mathrm{c}} M_{2} /\left(M_{\mathrm{c}}+M_{2}\right)$ is the reduced mass of the system after explosion. The parameters $a_{\mathrm{f}}$ and $e$ are found by equating the total energy and the absolute value of the orbital momentum of the initial circular orbit to those of the resulting elliptical orbit (see Eqs. (13, $17,15))$ :

$$
\begin{aligned}
\mu_{\mathrm{f}} \frac{V_{\mathrm{f}}^{2}}{2}-\frac{G M_{\mathrm{c}} M_{2}}{a_{\mathrm{i}}} & =-\frac{G M_{\mathrm{c}} M_{2}}{2 a_{\mathrm{f}}}, \\
\mu_{\mathrm{f}} a_{\mathrm{i}} \sqrt{w_{z}^{2}+\left(V_{\mathrm{i}}+w_{y}\right)^{2}} & =\mu_{\mathrm{f}} \sqrt{G\left(M_{\mathrm{c}}+M_{2}\right) a_{\mathrm{f}}\left(1-e^{2}\right)} .
\end{aligned}
$$

For the resulting $a_{\mathrm{f}}$ and $e$ one finds

$$
\frac{a_{\mathrm{f}}}{a_{\mathrm{i}}}=\left[2-\chi\left(\frac{w_{x}^{2}+w_{z}^{2}+\left(V_{\mathrm{i}}+w_{y}\right)^{2}}{V_{\mathrm{i}}^{2}}\right)\right]^{-1}
$$

and

$$
1-e^{2}=\chi \frac{a_{\mathrm{i}}}{a_{\mathrm{f}}}\left(\frac{w_{z}^{2}+\left(V_{\mathrm{i}}+w_{y}\right)^{2}}{V_{\mathrm{i}}^{2}}\right),
$$

where $\chi \equiv\left(M_{1}+M_{2}\right) /\left(M_{\mathrm{c}}+M_{2}\right) \geq 1$. The angle $\theta$ is defined by

$$
\cos \theta=\frac{\overrightarrow{J_{\mathrm{f}}} \cdot \overrightarrow{J_{\mathrm{i}}}}{\left|\overrightarrow{J_{\mathrm{f}}}\right|\left|\overrightarrow{J_{\mathrm{i}}}\right|}
$$

which results in

$$
\cos \theta=\frac{V_{\mathrm{i}}+w_{y}}{\sqrt{w_{z}^{2}+\left(V_{\mathrm{i}}+w_{y}\right)^{2}}} .
$$


The condition for disruption of the binary system depends on the absolute value $V_{\mathrm{f}}$ of the final velocity, and on the parameter $\chi$. The binary disrupts if its total energy defined by the lefthand-side of Eq. (44) becomes non-negative or, equivalently, if its eccentricity defined by Eq. (47) becomes $e \geq 1$. From either of these requirements one derives the condition for disruption:

$$
\frac{V_{\mathrm{f}}}{V_{\mathrm{i}}} \geq \sqrt{\frac{2}{\chi}} .
$$

The system remains bound if the opposite inequality is satisfied. Eq. (49) can also be written in terms of the escape (parabolic) velocity $V_{\mathrm{e}}$ defined by the requirement

$$
\mu_{\mathrm{f}} \frac{V_{\mathrm{e}}^{2}}{2}-\frac{G M_{\mathrm{c}} M_{2}}{a_{\mathrm{i}}}=0 .
$$

Since $\chi=M /(M-\Delta M)$ and $V_{\mathrm{e}}^{2}=2 G(M-\Delta M) / a_{\mathrm{i}}=2 V_{\mathrm{i}}^{2} / \chi$, one can write Eq. (49) in the form

$$
V_{\mathrm{f}} \geq V_{\mathrm{e}} .
$$

The condition of disruption simplifies in the case of a spherically-symmetric SN explosion, that is, when there is no kick velocity, $\vec{w}=0$, and, therefore, $V_{\mathrm{f}}=V_{\mathrm{i}}$. In this case, Eq. (49) reads $\chi \geq 2$, which is equivalent to $\Delta M \geq M / 2$. Thus, the system unbinds if more than half of the mass of the binary is lost. In other words, the resulting eccentricity

$$
e=\frac{M_{1}-M_{\mathrm{c}}}{M_{\mathrm{c}}+M_{2}}
$$

following from Eqs. (46) and (47), and $\vec{w}=0$ becomes larger than 1, if $\Delta M>M / 2$.

So far, we have considered an originally circular orbit. If the pre-SN star moves in an originally eccentric orbit, the condition of disruption of the system under symmetric explosion reads

$$
\Delta M=M_{1}-M_{\mathrm{c}}>\frac{1}{2} \frac{r}{a_{\mathrm{i}}},
$$

where $r$ is the distance between the components at the moment of explosion.

\subsection{Kick velocity of neutron stars}

The kick imparted to a NS at birth is one of the major problems in the theory of stellar evolution. By itself, it is an additional parameter, the introduction of which has been motivated first of all by high space velocities of radio pulsars inferred from the measurements of their proper motions and distances. Pulsars were recognized as a high-velocity Galactic population soon after their discovery in 1968 [256]. Shklovskii [704] put forward the idea that high pulsar velocities may result from asymmetric supernova explosions. Since then this hypothesis has been tested by pulsar observations, but no definite conclusions on its magnitude and direction have as yet been obtained.

Indeed, the distance to a pulsar is usually derived from the dispersion measure evaluation and crucially depends on the assumed model of electron density distribution in the Galaxy. In the middle of the 1990s, Lyne and Lorimer [449] derived a very high mean space velocity of pulsars with known proper motion of about $450 \mathrm{~km} \mathrm{~s}^{-1}$. This value was difficult to adopt without invoking an additional natal kick velocity of NSs.

The high mean space velocity of pulsars, consistent with earlier results by Lyne and Lorimer, was confirmed by the analysis of a larger sample of pulsars [284]. The recovered distribution of $3 \mathrm{D}$ velocities is well fit by a Maxwellian distribution with the mean value $w_{0}=400 \pm 40 \mathrm{~km} \mathrm{~s}^{-1}$ and a $1 \mathrm{D} \mathrm{rms} \sigma=265 \mathrm{~km} \mathrm{~s}^{-1}$. 
Possible physical reasons for natal NS kicks due to hydrodynamic effects in core-collapse supernovae are summarized in [402, 401]. Large kick velocities $\left(\sim 500 \mathrm{~km} \mathrm{~s}^{-1}\right.$ and even more) imparted to nascent NSs are generally confirmed by detailed numerical simulations (see, e.g., [536, 846]). Neutrino effects in the strong magnetic field of a young NS may also be essential in explaining kicks up to $\sim 100 \mathrm{~km} \mathrm{~s}^{-1}[109,160,398]$. Astrophysical arguments favoring a kick velocity are also summarized in [754]. To get around the theoretical difficulty of insufficient rotation of pre-supernova cores in single stars to produce rapidly-spinning young pulsars, Spruit and Phinney [731] proposed that random off-center kicks can lead to a net spin-up of proto-NSs. In this model, correlations between pulsar space velocity and rotation are possible and can be tested in further observations.

Here we should note that the existence of some kick follows not only from the measurements of radio pulsar space velocities, but also from the analysis of binary systems with NSs. The impact of a kick velocity $\sim 100 \mathrm{~km} \mathrm{~s}^{-1}$ explains the precessing pulsar binary orbit in PSR J0045-7319 [345]. The evidence of the kick velocity is seen in the inclined, with respect to the orbital plane, circumstellar disk around the Be star SS 2883 - an optical component of a binary PSR B1259-63 [617]. Evidence for $\sim 150 \mathrm{~km} \mathrm{~s}^{-1}$ natal kicks has also been inferred from the statistics of the observed short GRB distributions relative to their host galaxies [31].

Long-term pulse profile changes interpreted as geodetic precession are observed in the relativistic pulsar binaries PSR 1913+16 [831], PSR B1534+12 [734], PSR J1141-6545 [289], and PSR J0737-3039B [78]. These observations indicate that in order to produce the misalignment between the orbital angular momentum and the neutron star spin, a component of the kick velocity perpendicular to the orbital plane is required [835, 839, 840]. This idea seems to gain observational support from recent thorough polarization measurements [324] suggesting alignment of the rotational axes with the pulsar's space velocity. Detailed discussion of the spin-velocity alignment in young pulsars and implications for the SN kick mechanisms can be found in Noutsos et al. [538]. Such an alignment acquired at birth may indicate the kick velocity directed preferably along the rotation of the proto-NS. For the first SN explosion in a close binary system this would imply that the kick is mostly perpendicular to the orbital plane. Implications of this effect for the formation and coalescence rates of NS binaries were discussed by Kuranov et al. [396].

It is worth noting that the analysis of the formation of the relativistic pulsar binaryPSR J07373039 [595] may suggest, from the observed low eccentricity of the system $e \simeq 0.09$, that a small (if any) kick velocity may be acquired if the formation of the second NS in the system is associated with the collapse of an ONeMg WD due to electron captures. The symmetric nature of electroncapture supernovae was discussed in [597] and seems to be an interesting issue requiring further studies (see, e.g., [582, 397] for the analysis of the formation of NSs in globular clusters in the frame of this hypothesis). Note that electron-capture SNe are expected to be weak events, irrespective of whether they are associated with the core collapse of a star that retained some original envelope or with the AIC of a WD [644, 366, 146]. In the case of AIC, rapid rotation of a collapsing object along with flux freezing and dynamo action can grow the WD's magnetic field to magnetar strengths during collapse. Further, magnetar generates outflow of the matter and formation of a pulsar wind nebula, which may be observed as a radio-source for a few month [592].

We also note the hypothesis of Pfahl et al. [583], based on observations of high-mass X-ray binaries with long orbital periods $(\gtrsim 30 \mathrm{~d})$ and low eccentricities $(e<0.2)$, that rapidly rotating precollapse cores may produce neutron stars with relatively small kicks, and vice versa for slowly rotating cores. Then, large kicks would be a feature of stars that retained deep convective envelopes long enough to allow a strong magnetic torque, generated by differential rotation between the core and the envelope, to spin the core down to the very slow rotation rate of the envelope. A low kick velocity imparted to the second (younger) neutron star $\left(<50 \mathrm{~km} \mathrm{~s}^{-1}\right)$ was inferred from the analysis of large-eccentricity pulsar binary PSR J1811-1736 [119]. The large orbital period of this pulsar binary (18.8 d) may then suggest an evolutionary scenario with inefficient (if any) common envelope stage [148], i.e., the absence of a deep convective shell in the supernova progenitor (a 
He-star). This conclusion can be regarded as supportive to ideas put forward by Pfahl et al. [583]. A careful investigation of radio profiles of double PSR J0737-3039A/B [198] and Fermi detection of gamma-ray emission from the recycled 22-ms pulsar [254] imply its spin axis to be almost aligned with the orbital angular momentum, which lends further credence to the hypothesis that the second supernova explosion in this system was very symmetric.

Small kicks in the case of e-capture in the O-Ne core leading to NS formation are justified by hydrodynamical considerations. Indeed, already in 1996, Burrows and Hayes [80] noted that large scale convective motions in $\mathrm{O}$ and $\mathrm{Si}$ burning stages preceding the formation of a Fe core may produce inhomogeneities in the envelope of the protostellar core. They in turn may result in asymmetric neutrino transport, which impart kicks up to $500 \mathrm{~km} \mathrm{~s}^{-1}$. Such a violent burning does not precede the formation of O-Ne cores and then small or even zero kicks can be expected.

In the case of asymmetric core-collapse supernova explosion, it is natural to expect some kick during $\mathrm{BH}$ formation as well $[430,214,607,609,518,34,872]$. The similarity of NS and BH distribution in the Galaxy suggesting BH kicks was noted in [327]. Evidence for a moderate $\left(100-200 \mathrm{~km} \mathrm{~s}^{-1}\right)$ BH kick has been derived from the kinematics of several BH X-ray transients (microquasars): XTE J1118+180 [208], GRO 1655-40 [838], MAXI J1659-152 [400]. However, no kicks or only small ones seem to be required to explain the formation of other $\mathrm{BH}$ candidates, such as Cyg X-1 [485], [845], X-Nova Sco [514], V404 Cyg [484]. Population synthesis modeling of the Galactic distribution of $\mathrm{BH}$ binaries supports the need for (possibly, bimodal) natal BH kicks [634]. Janka [321] argued that the similarity of BH kick distribution with NS kick distributions as inferred from the analysis by Repetto et al. [634] favors BH kicks being due to gravitational interaction with asymmetric mass ejection (the "gravitational tug-boat mechanism"), and disfavors neutrino-induced kicks (in the last case, by momentum conservation, BH kicks are expected to be reduced by the NS to the $\mathrm{BH}$ mass ratio relative to the NS kicks). Facing current uncertainties in $\mathrm{SN}$ explosions and $\mathrm{BH}$ formation mechanisms, it is not excluded that low-kick BHs can be formed without associated SN explosions due to neutrino asymmetry, while high-velocity Galactic BHs in LMXBs analyzed by Repetto et al. [634] can be formed by the gravitational tug-boat mechanism suggested by Janka [321].

To summarize, the kick velocity remains to be one of the important unknown parameters of binary evolution with NSs and BHs, and further phenomenological input here is of great importance. Large kick velocities will significantly affect the spatial distribution of coalescing compact binaries (e.g., [352]) and BH kicks are extremely important for BH spin misalignment in coalescing BH-BH binary systems (e.g., Gerosa et al. [237] and references therein). Further constraining this parameter with various observations and a theoretical understanding of the possible asymmetry of core-collapse supernovae seem to be of paramount importance for the formation and evolution of close compact binaries.

\subsubsection{Effect of the kick velocity on the evolution of a binary system}

The collapse of a star to a $\mathrm{BH}$, or its explosion leading to the formation of a NS, are normally considered as instantaneous. This assumption is well justified in binary systems, since typical orbital velocities before the explosion do not exceed a few hundred $\mathrm{km} / \mathrm{s}$, while most of the mass is expelled with velocities of about several thousand $\mathrm{km} / \mathrm{s}$. The exploding star $M_{1}$ leaves the remnant $M_{\mathrm{c}}$, and the binary loses a part of its mass: $\Delta M=M_{1}-M_{\mathrm{c}}$. The relative velocity of stars before the event is

$$
V_{\mathrm{i}}=\sqrt{G\left(M_{1}+M_{2}\right) / a_{\mathrm{i}}} .
$$

Right after the event, the relative velocity is

$$
\vec{V}_{\mathrm{f}}=\vec{V}_{\mathrm{i}}+\vec{w} .
$$


Depending on the direction of the kick velocity vector $\vec{w}$, the absolute value of $\vec{V}_{\mathrm{f}}$ varies in the interval from the smallest $V_{\mathrm{f}}=\left|V_{\mathrm{i}}-w\right|$ to the largest $V_{\mathrm{f}}=V_{\mathrm{i}}+w$. The system gets disrupted if $V_{\mathrm{f}}$ satisfies the condition (see Section 3.4)

$$
V_{\mathrm{f}} \geq V_{\mathrm{i}} \sqrt{\frac{2}{\chi}}
$$

where $\chi \equiv\left(M_{1}+M_{2}\right) /\left(M_{\mathrm{c}}+M_{2}\right)$.

Let us start from the limiting case when the mass loss is practically zero $(\Delta M=0, \chi=1)$, while a non-zero kick velocity can still be present. This situation can be relevant to $\mathrm{BH}$ formation. It follows from Eq. (54) that, for relatively small kicks, $w<(\sqrt{2}-1) V_{\mathrm{i}}$, the system always (independent of the direction of $\vec{w})$ remains bound, while for $w>(\sqrt{2}+1) V_{\mathrm{i}}$ the system always decays. By averaging over equally probable orientations of $\vec{w}$ with a fixed amplitude $w$, one can show that in the particular case $w=V_{\mathrm{i}}$ the system disrupts or survives with equal probabilities. If $V_{\mathrm{f}}<V_{\mathrm{i}}$, the semi-major axis of the system becomes smaller than the original binary separation, $a_{\mathrm{f}}<a_{\mathrm{i}}$ (see Eq. (46)). This means that the system becomes harder than before, i.e. it has a greater negative total energy than the original binary. If $V_{\mathrm{i}}<V_{\mathrm{f}}<\sqrt{2} V_{\mathrm{i}}$, the system remains bound, but $a_{\mathrm{f}}>a_{\mathrm{i}}$. For small and moderate kicks $w \gtrsim V_{\mathrm{i}}$, the probabilities for the system to become more or less bound are approximately equal.

In general, the binary system loses some fraction of its mass $\Delta M$. In the absence of the kick, the system remains bound if $\Delta M<M / 2$ and gets disrupted if $\Delta M \geq M / 2$ (see Section 3.4). Clearly, a "properly" oriented kick velocity (directed against the vector $\vec{V}_{\mathrm{i}}$ ) can keep the system bound, even if it would have been disrupted without the kick. And, on the other hand, an "unfortunate" direction of $\vec{w}$ can disrupt the system, which otherwise would stay bound.

Consider, first, the case $\Delta M<M / 2$. The parameter $\chi$ varies in the interval from 1 to 2 , and the escape velocity $V_{\mathrm{e}}$ varies in the interval from $\sqrt{2} V_{\mathrm{i}}$ to $V_{\mathrm{i}}$. It follows from Eq. (50) that the binary always remains bound if $w<V_{\mathrm{e}}-V_{\mathrm{i}}$, and always unbinds if $w>V_{\mathrm{e}}+V_{\mathrm{i}}$. This is a generalization of the formulas derived above for the limiting case $\Delta M=0$. Obviously, for a given $w$, the probability for the system to disrupt or become softer increases when $\Delta M$ becomes larger. Now turn to the case $\Delta M>M / 2$. The escape velocity of the compact star becomes $V_{\mathrm{e}}<V_{\mathrm{i}}$. The binary is always disrupted if the kick velocity is too large or too small: $w>V_{\mathrm{i}}+V_{\mathrm{e}}$ or $w<V_{\mathrm{i}}-V_{\mathrm{e}}$. However, for all intermediate values of $w$, the system can remain bound, and sometimes even more hard than before, if the direction of $\vec{w}$ happened to be approximately opposite to $\vec{V}_{\mathrm{i}}$. A detailed calculation of the probabilities for the binary survival or disruption requires integration over the kick velocity distribution function $f(\vec{w})$ (see, e.g., [68]).

\subsection{Common envelope stage}

\subsubsection{Formation of the common envelope}

Common envelopes (CE) are definitely the most important (and not as yet solved) problem in the evolution of close binaries. In the theory of stellar evolution, it was recognized quite early that there are several situations when formation of an envelope that engulfs an entire system seems to be inevitable. It can happen when the mass transfer rate from the mass-losing star is so high that the companion cannot accommodate all the accreting matter [41, 868, 631, 523, 823, 614, 590]. Another instance is encountered when it is impossible to keep synchronous rotation of a red giant and orbital revolution of a compact companion [730, 8]. Because of tidal drag forces, the revolution period decreases, while the rotation period increases in order to reach synchronism (Darwin instability). If the total orbital momentum of the binary $J_{\mathrm{orb}}<3 J_{\mathrm{RG}}$, the synchronism cannot be reached and the companion (low-mass star, white dwarf or neutron star) spirals into the envelope of the red giant. Yet another situation is the formation of an extended envelope, which enshrouds the system due 
to unstable nuclear burning at the surface of an accreting WD in a compact binary [738, 559, 534]. It is also possible that a compact remnant of a supernova explosion with "appropriately" directed kick velocity finds itself in an elliptic orbit whose minimum periastron distance $a_{\mathrm{f}}(1-e)$ is smaller than the radius of the companion.

The common-envelope stage appears to be unavoidable on observational grounds. The evidence for a dramatic orbital angular momentum decrease in the course of evolution follows immediately from observations of cataclysmic variables, in which a white dwarf accretes matter from a small red dwarf main-sequence companion, close binary nuclei of planetary nebulae, low-mass X-ray binaries, and X-ray transients (neutron stars and black holes accreting matter from low-mass main-sequence dwarfs). At present, the typical separation of components in these systems is $\sim R_{\odot}$, while the formation of compact stars requires progenitors with radii $\sim(100-1000) R_{\odot} \cdot{ }^{17}$ Indirect evidence for the common envelope stage comes, for instance, from X-ray and FUV observations of a prototypical pre-cataclysmic binary V471 Tau showing anomalous $\mathrm{C} / \mathrm{N}$ contamination of the K-dwarf companion to white dwarf [164, 714].

Let us consider the following mass-radius exponents that describe the response of a star to the mass loss in a binary system:

$$
\zeta_{L}=\left(\frac{\partial \ln R_{1}}{\partial \ln M_{1}}\right), \quad \zeta_{\mathrm{th}}=\left(\frac{\partial \ln R_{L}}{\partial \ln M_{1}}\right)_{\mathrm{th}}, \quad \zeta_{\mathrm{ad}}=\left(\frac{\partial \ln R_{L}}{\partial \ln M_{1}}\right)_{\mathrm{ad}},
$$

where $\zeta_{L}$ is the response of the Roche lobe to the mass loss, $\zeta_{\text {th }}$ - the thermal-equilibrium response, and $\zeta_{\mathrm{ad}}$ - the adiabatic hydrostatic response. If $\zeta_{\mathrm{ad}}>\zeta_{L}>\zeta_{\mathrm{th}}$, the star retains hydrostatic equilibrium, but does not remain in thermal equilibrium and mass loss occurs in the thermal timescale of the star. If $\zeta_{L}>\zeta_{\text {ad }}$, the star cannot retain hydrostatic equilibrium and mass loss proceeds in the dynamical time scale [558]. If both $\zeta_{\text {ad }}$ and $\zeta_{\text {th }}$ exceed $\zeta_{L}$, mass loss occurs due to expansion of the star caused by nuclear burning or due to the shrinkage of the Roche lobe owed to the angular momentum loss.

A high rate of mass overflow onto a compact star from a normal star is always expected when the normal star goes off the main sequence and develops a deep convective envelope. The physical reason for this is that convection tends to make entropy constant along the radius, so the radial structure of convective stellar envelopes is well described by a polytrope (i.e., the equation of state can be written as $P=K \rho^{1+1 / n}$ ) with an index $n=3 / 2$. The polytropic approximation with $n=3 / 2$ is also valid for degenerate white dwarfs with masses not too close to the Chandrasekhar limit. For a star in hydrostatic equilibrium, this results in the inverse mass-radius relation, $R \propto M^{-1 / 3}$, first found for white dwarfs. Removing mass from a star with a negative power of the mass-radius relation increases its radius. On the other hand, the Roche lobe of the more massive star should shrink in response to the conservative mass exchange between the components. This further increases the mass loss rate from the Roche-lobe filling star leading to dynamical mass loss and eventual formation of a common envelope. If the star is completely convective or completely degenerate, dynamically-unstable mass loss occurs if the mass ratio of components (donor to accretor) is $\gtrsim 2 / 3$.

In a more realistic case when the star is not completely convective and has a radiative core, certain insight may be gained by the analyses of composite polytropic models. Conditions for the onset of dynamical mass loss become less rigorous [555, 283, 722]: the contraction of a star replaces expansion if the relative mass of the core $m_{c}>0.214$ and in a binary with a mass ratio of components close to 1, a Roche-lobe filling star with a deep convective envelope may remain dynamically stable if $m_{c}>0.458$. As well, a stabilizing effect upon mass loss may have mass and momentum loss from the system, if it happens in a mode that results in an increase of the

\footnotetext{
17 In principle, it is possible to form a close binary system with a compact star (NS or BH) from a hierarchical triple system without an initial CE phase due to dynamical interactions (Kozai-Lidov mechanism), see [693].
} 
specific angular momentum of the binary (e.g., in the case of isotropic reemission by a more massive component of the system in CVs or UCXBs).

Criteria for thermal or dynamical mass loss upon RLOF and the formation of a common envelope need systematic exploration of the response of stars to mass removal in different evolutionary stages and at different rates. The response also depends on the mass of the star. While computational methods for such an analyses are elaborated [224, 225], calculations of respective grids of models with full-fledged evolutionary codes at the time of writing of this review were not completed.

\subsection{2 "Alpha"-formalism}

Formation of the common envelope and evolution of a binary inside the former is a 3D hydrodynamic process that may also include nuclear reactions; and current understanding of the process as a whole, as well as computer power, are not sufficient for a solution of the problem (see, for detailed discussion, [319]). Therefore, the outcome of this stage is, most commonly, evaluated in a simplified way, based on the balance of the binding energy of the stellar envelope and the binary orbital energy, following independent suggestions of van den Heuvel [794], Tutukov and Yungelson [783], and Webbink [825] and commonly named after the later author as "Webbink's" or " $\alpha$ "-formalism. The orbital evolution of the compact star $m$ inside the envelope of the donor-star $M_{1}$ is driven by dynamic friction drag [553]. This leads to a gradual spiral-in process of the compact star. The above-mentioned energy condition may be written as

$$
\frac{G M_{1}\left(M_{1}-M_{c}\right)}{\lambda R_{\mathrm{L}}}=\alpha_{\mathrm{ce}}\left(\frac{G m M_{c}}{2 a_{\mathrm{f}}}-\frac{G M_{1} m}{2 a_{\mathrm{i}}}\right),
$$

where $a_{\mathrm{i}}$ and $a_{\mathrm{f}}$ are the initial and final orbital separations, $\alpha_{\mathrm{ce}}$ is the common envelope parameter [783, 438] that describes the efficiency of expenditure of orbital energy on expulsion of the envelope and $\lambda$ is a numerical coefficient that depends on the structure of the donor's envelope, introduced by de Kool et al. [138], and $R_{\mathrm{L}}$ is the Roche lobe radius of the normal star that can be approximated, e.g., by Eqs. (34) or (35). From Eq. (56) one derives

$$
\frac{a_{\mathrm{f}}}{a_{\mathrm{i}}}=\frac{M_{c}}{M_{1}}\left(1+\frac{2 a_{\mathrm{i}}}{\lambda \alpha_{\mathrm{CE}} R_{\mathrm{L}}} \frac{M_{1}-M_{c}}{m}\right)^{-1} \lesssim \frac{M_{c}}{M_{1}} \frac{m}{\Delta M},
$$

where $\Delta M=M_{1}-M_{c}$ is the mass of the ejected envelope. For instance, the mass $M_{c}$ of the helium core of a massive star can be approximated as [782]

$$
M_{\mathrm{He}} \approx 0.1\left(M_{1} / M_{\odot}\right)^{1.4} .
$$

Then, $\Delta M \sim M$ and, e.g., in the case of $m \sim M_{\odot}$ the orbital separation during the common envelope stage can decrease by as much as a factor of 40 or even more.

This treatment does not take into account possible transformations of the binary components during the CE-stage. In addition, the pre-CE evolution of the binary components may be important for the onset and the outcome of the $\mathrm{CE}$, as calculations of synchronization of red giant stars in close binaries carried out by Bear and Soker [29] indicate. The outcome of the common envelope stage is considered as a merger of components if $a_{f}$ is such that the donor core comes into contact with the companion. Otherwise, it is assumed that the core and companion form a detached system with the orbital separation $a_{f}$. Note an important issue raised recently by Kashi and Soker [341]: while Eq. (57) can formally imply that the system becomes detached at the end of the CE stage, in fact, some matter of the envelope can not reach the escape velocity and remains bound to the system and forms a circumbinary disk. Angular momentum loss due to interaction with the disk may result in a further reduction of binary separation and merger of components. This may influence the features of such stellar populations as a close WD binary, hot subdwarfs, cataclysmic variables) and the rate of SN Ia (see Section 7). 
In the above equation for the common envelope, the outcome of the CE stage depends on the product of two parameters: $\lambda$, which is the measure of the binding energy of the envelope to the core prior to the mass transfer in a binary system, and $\alpha_{\mathrm{CE}}$, which is the common envelope efficiency itself. Evaluation of both parameters suffers from large physical uncertainties. For example, for $\lambda$, the most debatable issues are the accounting of the internal energy in the binding energy of the envelope and the definition of the core/envelope boundary itself. Some authors argue (see e.g., [315]) that enthalpy rather than internal energy should be included in the calculation of $\lambda$, which seems physically justifiable for convective envelopes ${ }^{18}$. We refer the interested reader to the detailed discussion in $[139,319]$.

There exist several sets of fitting formulas for $\lambda[856,855]$ or binding energy of the envelopes [446] based on detailed evolutionary computations for a range of stellar models at different evolutionary stages. But we note, that in both studies the same specific evolutionary code ev [601] was used and the core/envelope interface at location in the star with hydrogen abundance in the hydrogenburning shell $X=0.1$ was assumed; in our opinion, the latter assumption is not justified neither by any physical assumptions nor by evolutionary computations for Roche-lobe filling stars. Even if the inaccuracy of the latter assumption is neglected, it is possible to use these formulas only in population synthesis codes based on the evolutionary tracks obtained by ev code.

Our test calculations do not confirm the recent claim [313] that core/envelope interface, which also defines the masses of the remnants in all cases of mass-exchange, is close to the radius of the sonic velocity maximum in the hydrogen-burning shell of the mass-losing star at RLOF. As concerns parameter $\alpha_{\mathrm{CE}}$, the most critical issue is whether the sources other than orbital energy can contribute to the energy balance, which include, in particular, recombination energy [724], nuclear energy [299], or energy released by accretion onto the compact star. It should then be noted that sometimes imposed to Eq. (56) restriction $\alpha_{\mathrm{CE}} \leq 1$ can not reflect the whole complexity of the processes occurring in common envelopes. Although full-scale hydrodynamic calculations of a common-envelope evolution exist, (see, e.g., [747, 140, 636, 637, 571] and references therein), we stress again that the process is still very far from comprehension, especially, at the final stages.

Actually, the state of the common envelope problem is currently such that it is possible only to estimate the product $\alpha_{\mathrm{CE}} \lambda$ by modeling specific systems or well defined samples of objects corrected for observational selection effects, like it is done in [765].

\subsection{3 "Gamma"-formalism}

Nelemans et. al. [517] noted that the $\alpha$-formalism failed to reproduce parameters of several close white dwarf binaries known circa 2000. In particular, for close helium white dwarf binaries with known masses of both components, one can reconstruct the evolution of the system "back" to the pair of main-sequence progenitors of components, since there is a unique relationship between the mass of a white dwarf and the radius of its red giant progenitor [631], which is almost independent of the total mass of the star. Formation of close white dwarf binaries should definitely involve a spiral-in phase in the common envelope during the second episode of mass loss (i.e., from the red giant to the white dwarf remnant of the original primary in the system). In observed systems, mass ratios of components tend to concentrate to $M_{\text {bright }} / M_{\text {dim }} \simeq 1$. This means, that, if the first stage of mass transfer occurred through a common envelope, the separation of components did not reduce much, contrary to what is expected from Eq. (57). The values of $\alpha_{\mathrm{CE}} \lambda$, which appeared necessary for reproducing the observed systems, turned out to be negative, which means that the simple energy conservation law (57) is violated in this case. Instead, Nelemans et al. suggested

\footnotetext{
18 Indeed, to put a gas element of unit mass with specific volume $v$ at a distance $R$ around mass $M$ from infinity, the work equal to the gravitational potential $\phi$ should be done; but then one should heat up the gas element, which is equivalent to give it the specific internal energy $\epsilon$, and also do $P v$-work to empty space in the already present gas, hence $\phi+H$ is relevant in calculating the binding energy of an isentropic envelope, where $d H=P d v$.
} 
that the first stage of mass exchange, between a giant and a main-sequence star with comparable masses $(q \geq 0.5)$, can be described by what they called " $\gamma$-formalism", in which not the energy but the angular momentum $J$ is balanced and conservation of energy is implicitly implied, though formally this requires $\alpha_{\mathrm{CE}} \lambda>1$ :

$$
\frac{\delta J}{J}=\gamma \frac{\Delta M}{M_{\mathrm{tot}}}
$$

Here $\Delta M$ is the mass lost by the donor, $M_{\text {tot }}$ is the total mass of the binary system before formation of the common envelope, and $\gamma$ is a numerical coefficient. Similar conclusions were later reached by Nelemans and Tout [516] and van der Sluys et al. [803] who analyzed larger samples of binaries and used detailed fits to evolutionary models instead of simple "core-mass - stellar radius" relation applied by Nelemans et al. [517]. It turned out that combination of $\gamma=1.75$ for the first stage of mass exchange with $\alpha_{\mathrm{CE}} \lambda=2$ for the second episode of mass exchange enables, after taking into account selection effects, a satisfactory model for population of close binary WD with known masses. In fact, findings of Nelemans et al. and van der Sluys et al. confirmed the need for a loss of mass and momentum from the system to explain observations of binaries mentioned in Section 3.3. (Note that $\gamma=1$ corresponds to the loss of the angular momentum by a fast stellar wind, which always increases the orbital separation of the binary.) We stress that no physical process behind $\gamma$-formalism has been suggested as yet, and the model remains purely phenomenological and should be further investigated. ${ }^{19}$

We should note that " $\gamma$-formalism" was introduced under the assumption that stars with deep convective envelopes (giants) always lose mass unstably, i.e., $\dot{M}$ is high. As mentioned above, if the mass exchange is nonconservative, the associated angular momentum loss can increase the specific orbital angular momentum of the system $\propto \sqrt{a}$ and hence the orbital separation $a$. Then the increasing Roche lobe can accommodate the expanding donor, and the formation of a common envelope can be avoided. In fact, this happens if part of the energy released by accretion is used to expel the matter from the vicinity of the accretor (see, e.g., computations presented by King and Ritter [365], Beer et al. [30], Woods et al. [849] who assumed that reemission occurs). It is not excluded that such nonconservative mass-exchange with mass and momentum loss from the system may be the process underlying the " $\gamma$-formalism".

Nelemans and Tout [516], Zorotovic et al. [886] and De Marco et al. [139] attempted to estimate $\alpha_{\mathrm{CE}}$ using samples of close WD $+\mathrm{M}$-star binaries. It turned out that their formation can be explained both if $\alpha_{\mathrm{CE}} \lambda>0$ in Eq. (56) or if $\gamma \approx 1.5$ in Eq. (59). This is sometimes considered as an argument against the $\gamma$-formalism. But we note that in progenitors of WD $+\mathrm{M}$-star binaries the companions to giants are low-mass small-radius objects, which are quite different from $M \geq M_{\odot}$ companions to $\mathrm{WD}$ in progenitors of $\mathrm{WD}+\mathrm{WD}$ binaries and, therefore, the energetics of the evolution in $\mathrm{CE}$ in precursors of two types of systems can differ. In a recent population synthesis study of the post-common-envelope binaries aimed at comparing to SDSS stars and taking into account selection effects, Toonen and Nelemans [765] concluded that the best fit to the observations can be obtained for small universal $\alpha_{\mathrm{CE}} \lambda=0.25$ without the need to invoke the $\gamma$-formalism. However, they also note that for almost equal mass precursors of WD binaries the widening of the orbit in the course of mass transfer is needed (like in $\gamma$-formalism), while the formation of low mass-ratio WD+M-star systems requires diminishing of the orbital separation (as suggested by $\alpha$-formalism).

19 Attempts to apply the $\gamma$-formalism to both stages of mass exchange $(\gamma, \gamma$-formalism), which failed to reproduce evolution of close WD binary and criticized the $\gamma$-formalism, are unjustified extrapolation of the limited application of the formalism suggested by [517]. 


\subsection{Other notes on the $\mathrm{CE}$ problem}

A particular case of common envelopes ("double spiral-in") occurs when both components are evolved. It was suggested [520] that a common envelope is formed by the envelopes of both components of the system and the binding energy of them both should be taken into account, i.e., the outcome of CE must be found from a solution of an equation

$$
E_{\mathrm{bind}, 1}+E_{\mathrm{bind}, 2}=\alpha_{\mathrm{CE}}\left(E_{\mathrm{orb}, i}-E_{\mathrm{orb}, f}\right) .
$$

Formulations of the common envelope equation different from Eq. (56) are found in the literature (see, e.g., [139] for a review); $a_{\mathrm{f}} / a_{\mathrm{i}}$ similar to the values produced by Eq. (56) are then obtained for different $\alpha_{\mathrm{ce}} \lambda$ values.

Common envelope events are expected to be rare $\left(\lesssim 0.1 \mathrm{yr}^{-1}[791]\right)$ and short-lasting ( $\sim$ years). Thus, the binaries at the CE-stage itself are difficult to observe, despite energy being released at this stage comparable to the binding energy of a star, and the evidence for them comes from the very existence of compact object binaries, as described above in Section 3.6.1. Recently, it was suggested that luminous red transients - the objects with peak luminosity intermediate between the brightest Novae and SN Ia (see, e.g., [342]) - can be associated with CE [316, 792].

We recall also that the stability and timescale of mass-exchange in a binary depends on the mass ratio of components $q$, the structure of the envelope of the Roche-lobe filling star, and possible stabilizing effects of mass and momentum loss from the system [780, 283, 873, 261, 268, $318,196,76,849]$. We note especially recent claims [848, 570] that the local thermal timescale of the super-adiabatic layer existing over a convective envelope of giants may be shorter than the donor's dynamic timescale. As a result, giants may adjust to a high mass loss and retain their radii or even contract (instead of dramatically expanding, as expected in the adiabatic approximation $[224,225])$. As well, the response of stars to mass loss evolves in the course of the latter depending on whether the super-adiabatic layer may be removed. The recent discovery of young helium WD companions to blue stragglers in wide binaries ( $P_{\text {orb }}$ from 120 to 3010 days) in open cluster NGC 188 [248] supports the idea that in certain cases RLOF in wide systems may be stable. These considerations, which need further systematic exploration, may change the "standard" paradigm of the stability of mass exchange formulated in this section. 


\section{Evolutionary Scenario for Compact Binaries with Neu- tron Star or Black Hole Components}

\subsection{Compact binaries with neutron stars}

Compact binaries with NS and BH components are descendants of initially massive binaries with $M_{1} \gtrsim 8 M_{\odot}$. The scenario of the evolution of massive binaries from a pair of main-sequence stars to a relativistic binary consisting of NSs or BHs produced by core-collapse SN was independently elaborated by Tutukov and Yungelson $[782,472]^{20}$ and van den Heuvel et al. [801, 201]. This scenario is fully confirmed by more than 30 years of astronomical observations and is now considered as "standard".

Certain modifications to the original scenario were introduced after Pfahl et al. [583] noticed that Be/X-ray binaries harboring NSs fall into two classes: objects with highly eccentric orbits $(e=$ $0.3-0.9)$ and $P_{\text {orb }} \geq 30$ day and objects with $e<0.2$ and $P_{\text {orb }} \leq 30$ day. Almost simultaneously, van den Heuvel [796] noted that 5 out of 7 known neutron star binaries and one massive WD + NS system in the Galactic disk have $e<0.27$ and the measured or estimated masses of second-born neutron stars in most of these systems are close to $1.25 M_{\odot}$ (for recent confirmation of these observations see, e.g., [685, 369, 799, 198]). A neutron star would have $1.25 M_{\odot}$ mass if it is a product of an electron-capture supernova (ECSN), which was accompanied by the loss of binding energy equivalent to $\approx 0.2 M_{\odot}$. These discoveries lead van den Heuvel [796] to include ECSN in the evolutionary scenario for the formation of neutron star binaries. A distinct feature of NSs formed via ECNS is their low natal kicks (typically, a Maxwellian velocity distribution with $\sigma=(20-30) \mathrm{km} \mathrm{s}^{-1}$ is inferred instead of $\left.\sigma \sim 200 \mathrm{~km} \mathrm{~s}^{-1}\right)$.

In Figure 7 we present the "standard" evolutionary scenario for the formation of neutron star binaries. Other versions of this scenario may differ by the types of supernovae occurring in them or in the order of their formation, which is defined by the initial masses of components and the orbital period of the binary.

It is convenient to consider subsequent stages of the evolution of a binary system according to the physical state of its components, including phases of mass exchange between them.

1. Initially, the pair of high-mass OB main-sequence stars is detached and stars are inside their Roche lobes. Tidal interaction is very effective and the possible initial eccentricity vanishes before the primary star $M_{1}$ fills its Roche lobe. The duration of this stage is determined by the hydrogen burning time of the primary, more massive component, and typically is $<10 \mathrm{Myr}$ (for massive main-sequence stars, the time of core hydrogen burning is $t_{\text {nucl }} \propto M^{-2}$ ). The star burns out hydrogen in its central parts, so that a dense central helium core with mass $M_{\mathrm{He}} \simeq 0.1\left(M / M_{\odot}\right)^{1.4}$ forms by the time the star leaves the main sequence. The expected number of such binaries in the Galaxy is about $10^{4}$.

2. After core hydrogen exhaustion, the primary leaves the main-sequence and starts to expand rapidly. When its radius approaches the Roche lobe, mass transfer onto the secondary, less massive star, which still resides on the main-sequence, begins. Depending on the masses of the components and the evolutionary state of the donor, the mass-transfer may proceed via non-conservative but stable RLOF or via a common envelope. Even if the common envelope is avoided and the first mass exchange event proceeds on the thermal time scale of the donor $\tau_{\mathrm{KH}} \approx G M_{1}^{2} / R_{1} L_{1}$, its duration for typical stars is rather short, on the order of $10^{4} \mathrm{yr}$, so only several dozens of such binaries are expected to be present in the Galaxy.

20 Tutukov and Yungelson's paper [782] was published in 1973 prior to the discovery of the first pulsar binary PSR 1913+16 in 1974 [757, 292] and, though the evolutionary path was traced to a NS (or BH) binary, since no such objects were known at that time, it was suggested that all pairs of NSs are disrupted at the second NS formation. 


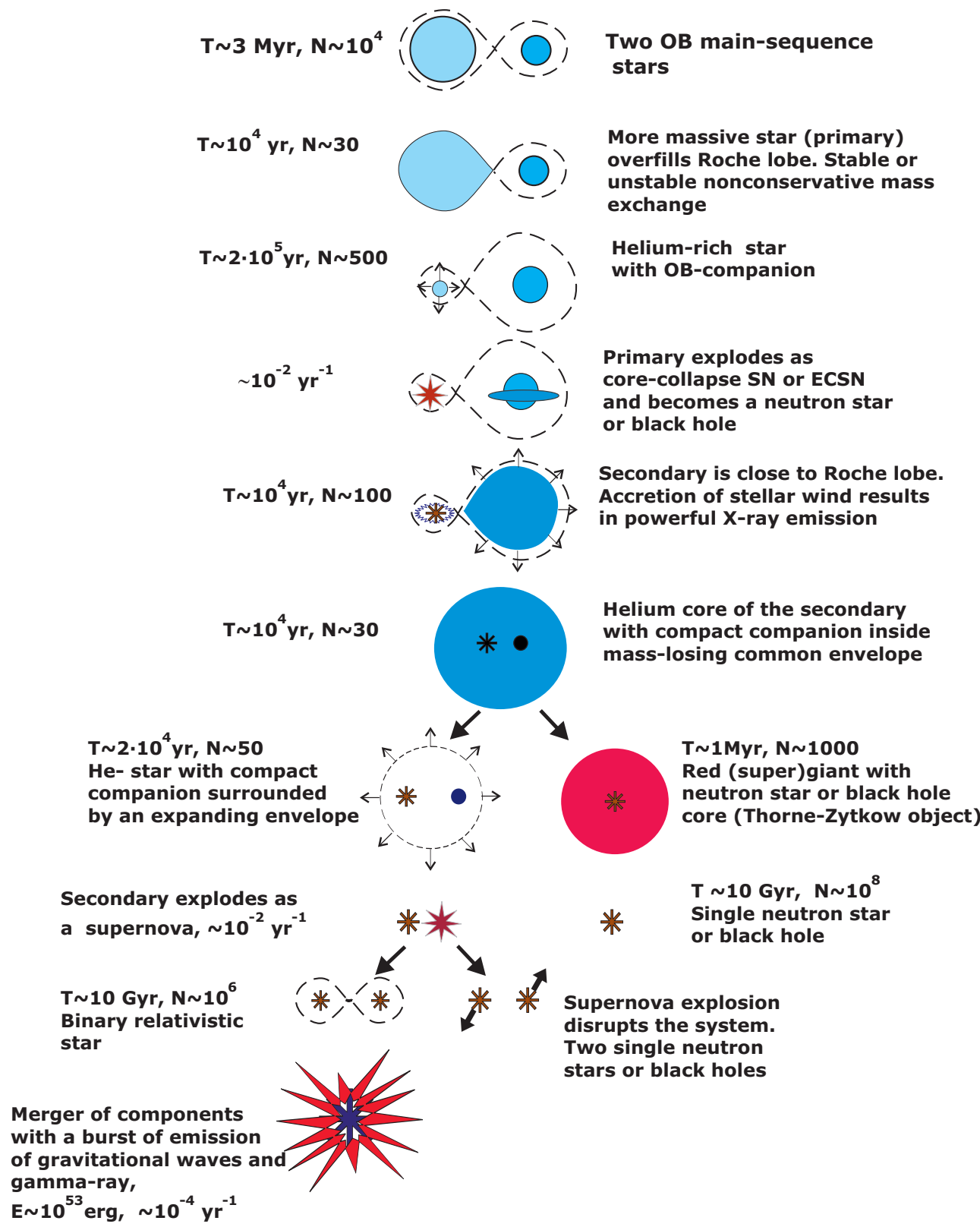

Figure 7: Evolutionary scenario for the formation of neutron stars or black holes in close binaries. $\mathrm{T}$ is the typical time scale of an evolutionary stage, $\mathrm{N}$ is the estimated number of objects in the given evolutionary stage. 
3. Mass transfer ends when most of the primary's hydrogen envelope is lost, so a naked helium core is left. This core can be observed as a Wolf-Rayet (WR) star with an intense stellar wind if its mass exceeds $(7-8) M_{\odot}[540,184,185]$. The duration of the WR stage is several $10^{5} \mathrm{yr}$, so the Galactic number of such binaries should be several hundreds.

During the mass-exchange episode the secondary star acquires a large angular momentum carried by the infalling matter, so that its outer envelope can be spun up to an angular velocity close to the limiting (Keplerian) value. Such massive rapidly-rotating stars are observed as Be-stars.

4. Stars more massive than $\simeq 8 M_{\odot}$ end their evolution by forming a NS. ZAMS mass range $8-12( \pm 1) M_{\odot}$, is a "transitional" one in which NS are formed via ECSN at the lower masses and via core collapses at the higher masses, as discussed in more detail in Section 1.

Supernovae associated with massive naked He stars (almost devoid of H-envelopes) are usually associated with SN Ib/c. The inferred Galactic-type SN Ib/c rate is $(0.76 \pm 0.16) \times$ $10^{-2} \mathrm{yr}^{-1}$ [417]. At least half of them should be in binaries. As mentioned in Section 1 ECSN may be progenitors of the faintest type II-P supernovae, because they produce only a small amount of radioactive Ni. Expected properties of core-collapse SN and ECSN are compared in Table 5 [685]. Peculiarly, the historical Crab SN in our Galaxy is suggested to be an ECSN [720].

Table 5: Comparison of Fe-Core Collapse and e-Capture Supernovae. Table reproduced with permission from [685], copyright by AAS.

\begin{tabular}{|c|c|c|}
\hline Properties & Iron Core Collapse & ECSN \\
\hline \multicolumn{3}{|l|}{ Supernova Properties } \\
\hline Explosion energy & $\sim 10^{51} \mathrm{ergs}$ & $\lesssim 10^{50}$ ergs \\
\hline Ejecta & rich in heavy elements $(\mathrm{Fe}, \mathrm{Si}, \mathrm{O})$ & few heavy elements \\
\hline \multicolumn{3}{|c|}{ Neutron Star Properties } \\
\hline Masses & range of masses & characteristic mass $\simeq 1.25 M_{\odot}$ \\
\hline Neutron star kick & large standard kick $\left(\sigma \simeq 265 \mathrm{~km} \mathrm{~s}^{-1}\right)$ & low kick \\
\hline \multicolumn{3}{|l|}{ Binary Properties } \\
\hline Occurrence & single or binaries & preferentially in binaries \\
\hline Eccentricity & high & low \\
\hline Recycled pulsar spin & $\begin{array}{l}\text { misaligned-aligned with orbit } \\
\text { (e.g., geodetic precession) }\end{array}$ & aligned with orbit \\
\hline
\end{tabular}

Disruption of the binary due to the second SN in the system is very likely (e.g., if the mass lost during the symmetric SN explosion exceeds 50\% of the total mass of the pre-SN binary, or it is even smaller in the presence of the kick; see Section 3.4 above). Population synthesis estimates show that $(4-10) \%$ of initial binaries survive the first core-collapse SN explosion in the system, depending on the assumed kick distribution [135, 607, 878, 396]. Some runaway Galactic OB-stars must have been formed in this way. Currently, only one candidate O-star with a non-interacting NS companion is known, thanks to multi-wavelength observations - HD 164816, a late O-type spectroscopic binary [772]. Null-results of earlier searches for similar objects $[585,675]$, though being dependent on assumptions on the beaming factor of 
pulsars and their magnetic field evolution, are consistent with a very low fraction of surviving systems, but may be also due to obscuration of radio emission by the winds of massive stars.

5. If the system survives the first $\mathrm{SN}$ explosion, a rapidly rotating Be star in orbit with a young NS appears. Orbital evolution following the SN explosion is described by Eqs. (46-51). The orbital eccentricity after the SN explosion is high, so enhanced accretion onto the NS occurs at the periastron passages. Most of about 100 Galactic Be/X-ray binaries [622] may be formed in this way. Post-ECSN binaries have a larger chance for survival thanks to low kicks. It is possible that a significant fraction of Be/X-ray binaries belong to this group of objects. The duration of Be/X-ray stage depends on the binary parameters, but in all cases it is limited by the time left for the (now more massive) secondary to burn hydrogen in its core.

An important parameter of NS evolution is the surface magnetic field strength. In binary systems, magnetic field, in combination with NS spin period and accretion rate onto the NS surface, determines the observational manifestation of the neutron star (see [423] for more details). Accretion of matter onto the NS can reduce the surface magnetic field and spin-up the NS rotation (pulsar recycling) [54, 654, 655, 52].

6. Evolving secondary expands to engulf the NS in its own turn. Formation of a common envelope is, apparently, inevitable due to the large mass ratio of the components. The common envelope stage after $\sim 10^{3}$ yr ends up with the formation of a WR star with a compact companion surrounded by an expanding envelope (Cyg X-3 may serve as an example), or the NS merges with the helium core during the common envelope to form a still hypothetical Thorne-Żytkow (TZ) object [759].

Cygnus X-3 - a WR star with a black hole or neutron star companion is unique in the Galaxy, because of the high probability of merger of the components in $\mathrm{CE}$, the short lifetime of surviving massive WR-stars and high velocity $\left(\sim 1000 \mathrm{~km} \mathrm{~s}^{-1}\right)$ of their stellar winds, which prevents the formation of accretion disks [177, 442, 422]. On the other hand, it is suggested that there may exist in the Galaxy a population of $\sim 100$ He-stars of between 1 and $7 M_{\odot}$ with relativistic companions, which do not reveal themselves, because these He-stars do not have strong-enough winds [442].

The possibility of the existence of TZ-stars remains unclear (see [25]). It was suggested that the merger products first become supergiants, but rapidly lose their envelopes due to heavy winds and become WR stars. Peculiar Wolf-Rayet stars of WN8 subtype were suggested as their observed counterparts [202]. These stars tend to have large spatial velocities, the overwhelming majority of them are single and they are the most variable among all single WR stars. The estimated observed number of them in the Galaxy is $\sim 10$. A single (possibly, massive) NS or BH should descend from them.

A note should be made concerning the phase when a common envelope engulfs the firstformed NS and the core of the secondary. Colgate [116] and Zel'dovich et al. [884] have shown that hyper-Eddington accretion onto a neutron star is possible if the gravitational energy released in accretion is lost by neutrinos. Chevalier [104] suggested that this may be the case for the accretion in common envelopes. Since the accretion rates in this case may be as high as $\sim 0.1 M_{\odot} \mathrm{yr}^{-1}$, the NS may collapse into a $\mathrm{BH}$ inside the common envelope. An essential caveat is that the accretion in the hyper-Eddington regime may be prevented by the angular momentum of the captured matter. The magnetic field of the NS may also be a complication. The possibility of hyper-critical accretion still has to be studied. Nevertheless, implications of this hypothesis for different types of relativistic binaries were explored in great detail by Bethe and Brown and their coauthors (see, e.g., [71] and references therein). Also, the possibility of hyper-Eddington accretion was included in several population synthesis 
studies with the evident result of diminishing the population of NS + NS binaries in favor of neutron stars in pairs with low-mass black holes (see, e.g., [607, 34]).

Recently, Chevalier [105] pointed to the possible connection of CE with a neutron star with some luminous and peculiar type IIn supernovae. The neutron star engulfed by the massive companion may serve as a trigger of the SN explosion in dense environments due to a violent mass-loss in the preceding CE phase. However, presently our understanding of the evolution of neutron stars inside CE is insufficient to test this hypothesis.

7. The secondary He-star ultimately explodes as a supernova leaving behind a NS binary, or the system disrupts to form two single high-velocity NSs or BHs. Even for a symmetric SN explosion the disruption of binaries after the second SN explosion could result in the observed high average velocities of radio pulsars (see Section 3.5 above). In the surviving close NS binary system, the older NS is expected to have a faster rotation velocity (and possibly higher mass) than the younger one because of the recycling at the preceding accretion stage. The subsequent orbital evolution of such NS binary systems is entirely due to GW emission (see Section 3.1.4) and ultimately leads to the coalescence of the components.

Detailed studies of possible evolutionary channels that produce merging NS binaries can be found in the literature, e.g., [787, 788, 430, 607, 19, 135, 834, 34, 294, 314, 815, 149, 839, 61, 332, $357,356,548,383,548,157,198])$. We emphasize that the above-described scenario applies only to close binaries, that have components massive enough to produce ECSN or core-collapse SN, but not so massive that the loss of the hydrogen envelope by stellar wind and an associated widening of the orbit via the Jeans mode of mass ejection may prevent RLOF by the primary. This limits the relevant mass range by $M_{1} \lesssim(40-50) M_{\odot}[470,807]$.

There also exists a population of NSs accompanied by low-mass $\left[\sim(1-2) M_{\odot}\right]$ companions. A scenario similar to the one presented in Figure 7 may be sketched for them too, with the difference that the secondary component stably transfers mass onto the companion (see, e.g., $[305,334,335,777])$. This scenario is similar to the one for low- and intermediate-mass binaries considered in Section 7, with the WD replaced by a NS or a BH. Compact low-mass binaries with NSs may be dynamically formed in dense stellar environments, for example in globular clusters. The dynamical evolution of binaries in globular clusters is beyond the scope of this review; see, e.g., [39] and [52] for more detail and further references.

At the end of numerical modeling of the evolution of massive binaries outlined above, one arrives at the population of NS binaries, the main parameter of which is distribution over orbital periods $P_{\text {orb }}$ and eccentricities $e$. In the upper panel of Figure 8 we show, as an example, a model of the probability distribution of Galactic NS + NS binaries with $P_{\text {orb }} \leq 10^{4}$ day in $P_{\text {orb }}-e$ plane at birth; the lower panel of the same figure shows a model probability distribution of the present day numbers of NS + NS systems younger than 10 Gyr [607]. It is clear that a significant fraction of systems born with $P_{\text {orb }} \leq 1$ day merge. A detailed discussion of general-relativity simulations of NS + NS mergers including the effects of magnetic fields and micro-physics is presented in [181].

As noted, important phases in the above described scenario are the stages in which one of the components is a WR-star. Evolution in close binaries is a channel for formation of a significant fraction of them, since RLOF is able to remove hydrogen envelopes from much lower-mass stars than stellar wind. As WR-stars are important contributors to the UV-light of the galaxies (as well as their lower-mass counterparts - hot subdwarfs), this points to the necessity of including binary evolution effects in the spectrophotometric-population-synthesis models, see, e.g., [419]. 

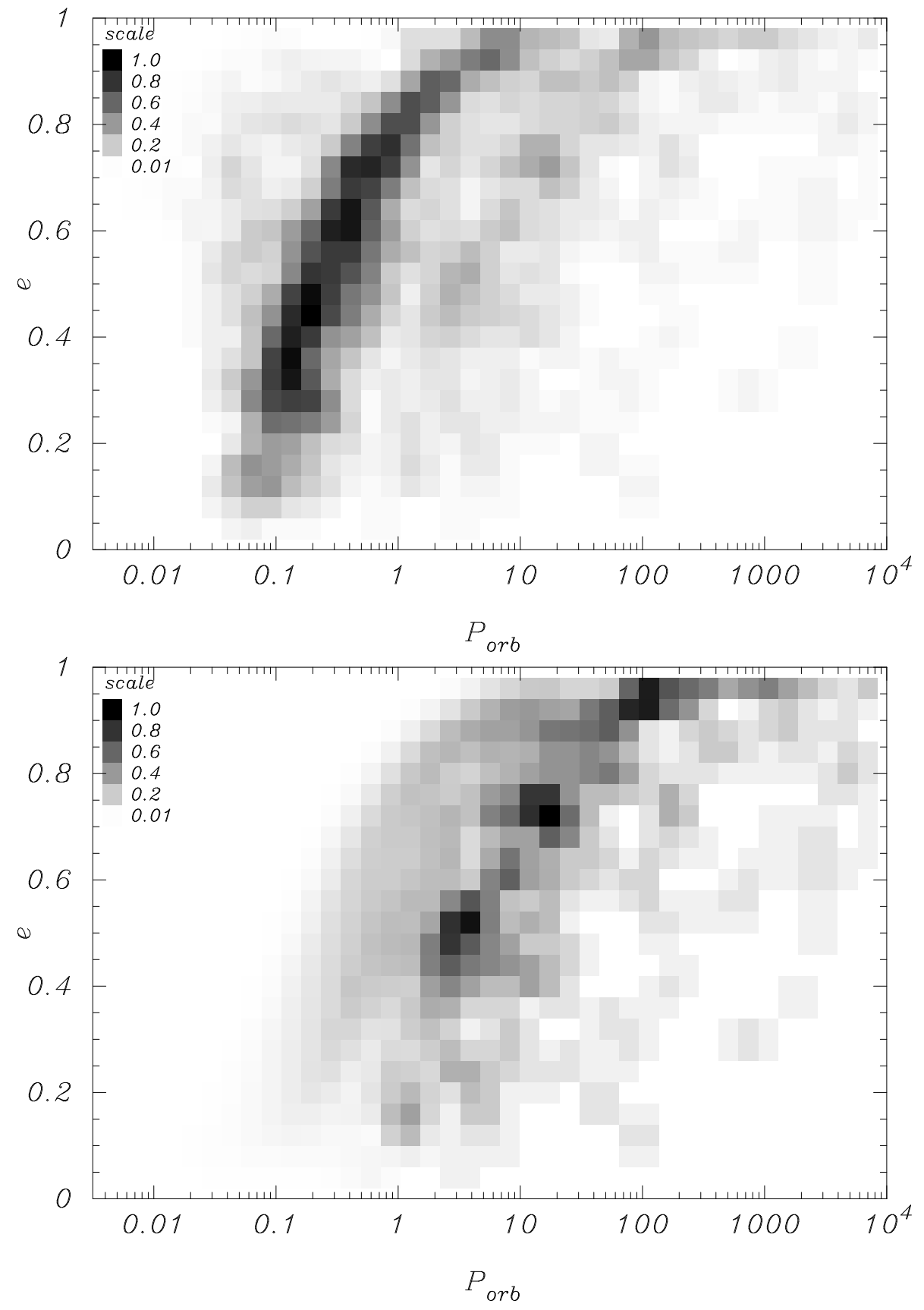

Figure 8: Upper panel: the probability distribution for the orbital parameters of the NS + NS binaries with $P_{\text {orb }} \leq 10^{4}$ day at the moment of birth. The darkest shade corresponds to a birthrate of $1.2 \times 10^{-5} \mathrm{yr}^{-1}$. Lower panel: the probability distribution for the present-day orbital parameters of the Galactic disc NS + NS binaries younger than $10 \mathrm{Gyr}$. The grey scaling represents numbers in the Galaxy. The darkest shade corresponds to 1100 binaries with given combination of $P_{\text {orb }}$ and $e$. 


\subsection{Black-hole-formation parameters}

So far, we have considered the formation of NSs and binaries with NSs. It is believed that very massive stars end their evolution by forming stellar-mass black holes. We will now discuss their formation.

In the analysis of $\mathrm{BH}$ formation, new important parameters appear. The first is the threshold mass $M_{\text {cr }}$ beginning from which a main-sequence star, after the completion of its nuclear evolution, can collapse into a BH. This mass is not well known; different authors suggest different values: van den Heuvel and Habets [802] - $40 M_{\odot}$; Woosley et al. [852] - $60 M_{\odot}$; Portegies Zwart, Verbunt, and Ergma [606], Ergma \& van den Heuvel [176], Brown et al. [70] - $20 M_{\odot}$. A simple physical argument usually put forward in the literature is that the mantle of the main-sequence star with $M>M_{\mathrm{cr}} \approx$ $30 M_{\odot}$ before the collapse has a binding energy well above $10^{51}$ erg (the typical supernova energy observed), so that the supernova shock is not strong enough to expel the mantle [210, 211].

The upper mass limit for BH formation (with the caveat that the role of magnetic-field effects is not considered) is, predominantly, a function of stellar-wind mass loss in the core-hydrogen, hydrogen-shell, and core-helium burning stages. For a specific combination of winds in different evolutionary stages and assumptions on metallicity it is possible to find the types of stellar remnants as a function of initial mass (see, for instance [274]). Since stellar winds are mass (or luminosity) and metallicity-dependent, a peculiar consequence of mass-loss implementation in the latter study is that for $Z \simeq Z_{\odot}$ the mass-range of precursors of black holes is constrained to $M \approx(25-60) M_{\odot}$, while more massive stars form NSs because of heavy mass loss. The recent discovery of the possible magnetar in the young stellar cluster Westerlund 1 [499] hints at the reality of such a scenario. Note, however, that the estimates of the stellar wind mass-loss rate $\dot{M}$ are rather uncertain, especially for the most massive stars, mainly because of clumping in the winds (see, e.g., [390, 123, 263]). Current reassessment of the role of clumping generally results in the reduction of previous massloss estimates. Other factors that have to be taken into account in the estimates of the masses of progenitors of BHs are rotation and magnetic fields.

The second parameter is the mass $M_{\mathrm{BH}}$ of the nascent $\mathrm{BH}$. There are various studies as for what the mass of the BH should be (see, e.g., [762, 44, 210, 215]). In some papers a typical BH mass was found to be not much higher than the upper limit for the NS mass (Oppenheimer-Volkoff limit $\sim(1.6-2.5) M_{\odot}$, depending on the unknown equation of state for NS matter) even if the fall-back accretion onto the supernova remnant is allowed [762]. Modern measurements of black hole masses in binaries suggest a broad range of them - $(4-17) M_{\odot}[542,475,633]$. A continuous range of $\mathrm{BH}$ masses up to $10-15 M_{\odot}$ was derived in calculations [215]. However, as stressed in Section 2.3, in view of the absence of robust "first-principle" calculations of stellar core collapses, both the BH progenitor's mass and that of the formed BH itself remain major parameters. Here many possibilities remain, including the interesting suggestion by Kochanek [371] that the absence of high mass giants $\left(16.5 M_{\odot}<M<25 M_{\odot}\right)$ as the progenitors of Type IIP supernovae may indicate $\mathrm{BH}$ formation starting from progenitor masses as low as $16 M_{\odot}$.

There is observational evidence that the dynamically-determined $\mathrm{BH}$ masses in extragalactic HMXBs (M 33 X-7, NGC 300 X-1, and IC10 X-1) residing in low-metallicity galaxies are higher $\left(16-30 M_{\odot}\right)$ than in the Milky Way surroundings [124]. Unless it is a selection effect (the brightest X-ray sources are observed first), this may indicate the dependence of $\mathrm{BH}$ formation on the progenitor's metallicity. This dependence is actually expected in the current models of evolution of single stars [235] and HMXB evolution [421].

It is still a challenge to reproduce successful supernova explosions in numerical calculations, especially in 3D (see, for example, [497], and the discussion in [568]). In the current numerical calculations, spectra of $\mathrm{BH}$ masses and kicks received by nascent BHs are still model dependent, see, e.g., [213, 321]. Therefore, in the further discussion we will parameterize the BH mass $M_{\mathrm{BH}}$ by the fraction of the pre-supernova mass $M_{*}$ that collapses into the $\mathrm{BH}: k_{\mathrm{BH}}=M_{\mathrm{BH}} / M_{*}$. In fact, the 
pre-supernova mass $M_{*}$ is directly related to $M_{\mathrm{cr}}$, but the form of this relationship is somewhat different in different scenarios for massive star evolution, mainly because of different mass-loss prescriptions. According to our parameterization, the minimal $\mathrm{BH}$ mass can be $M_{\mathrm{BH}}^{\min }=k_{\mathrm{BH}} M_{*}$, where $M_{*}$ itself depends on $M_{\mathrm{cr}}$. The parameter $k_{\mathrm{BH}}$ can vary in a wide range.

The third parameter, similar to the case of NS formation, is the possible kick velocity $\mathbf{w}_{\mathrm{BH}}$ imparted to the newly formed BH (see the end of Section 3.5). In general, one expects that the $\mathrm{BH}$ should acquire a smaller kick velocity than a NS, as black holes are more massive than neutron stars. A possible relation (as adopted, e.g., in calculations [430]) reads

$$
\frac{w_{\mathrm{BH}}}{w_{\mathrm{NS}}}=\frac{M_{*}-M_{\mathrm{BH}}}{M_{*}-M_{\mathrm{OV}}}=\frac{1-k_{\mathrm{BH}}}{1-M_{\mathrm{OV}} / M_{*}},
$$

where $M_{\mathrm{OV}}=2.5 M_{\odot}$ is the maximum NS mass. When $M_{\mathrm{BH}}$ is close to $M_{\mathrm{OV}}$, the ratio $w_{\mathrm{BH}} / w_{\mathrm{NS}}$ approaches 1, and the low-mass black holes acquire kick velocities similar to those of neutron stars. When $M_{\mathrm{BH}}$ is significantly larger than $M_{\mathrm{OV}}$, the parameter $k_{\mathrm{BH}}=1$, and the $\mathrm{BH}$ kick velocity becomes vanishingly small. The allowance for a quite moderate $w_{\mathrm{BH}}$ can increase the coalescence rate of $\mathrm{BH}$ binaries [430].

The possible kick velocity imparted to newly-born black holes makes the orbits of survived systems highly eccentric. It is important to stress that some fraction of such BH binaries can retain their large eccentricities up to the late stages of their coalescence. This signature should be reflected in their emitted waveforms and should be modeled in templates.

Asymmetric explosions accompanied by a kick change the space orientation of the orbital angular momentum. On the other hand, the star's spin axis remains fixed (unless the kick was off-center). As a result, some distribution of the angles between the $\mathrm{BH}$ spins and the orbital angular momentum (denoted by $J$ ) will be established [609, 331]. It is interesting that even for small kicks of a few tens of $\mathrm{km} / \mathrm{s}$ an appreciable fraction $(30-50 \%)$ of the merging $\mathrm{BH}$ binary can have $\cos J<0$. This means that in these binaries the orbital angular momentum vector is oriented almost oppositely to the black hole spin. This is one more signature of imparted kicks that can be tested observationally. The $\mathrm{BH}$ spin misalignment can have important consequences for BH-NS mergings [207]. The link between forthcoming observations by second and third-generation GW detectors with astrophysical scenarios of $\mathrm{BH}$ spin formation and evolution in compact binaries is discussed in more detail in [237]. 


\section{Formation of Double Compact Binaries}

\subsection{Analytical estimates}

A rough estimate of the formation rate of compact binaries can be obtained ignoring many details of binary evolution. To do this, we shall use the observed initial distribution of binary orbital parameters and assume the simplest conservative mass transfer $\left(M_{1}+M_{2}=\right.$ const) without kick velocity imparted to the nascent compact stellar remnants during SN explosions.

Initial binary distributions. From observations of binaries it is possible to derive their formation rate as a function of initial masses of components $M_{1}, M_{2}$ (with mass ratio $q=M_{2} / M_{1} \leq 1$ ), orbital semi-major axis $A$, and eccentricity $e$. According to [604, 811], the present birth rate of binaries in our Galaxy can be written in factorized form as

$$
\frac{d N}{d A d M_{1} d q d t} \approx 0.087\left(\frac{A}{R_{\odot}}\right)^{-1}\left(\frac{M_{1}}{M_{\odot}}\right)^{-2.5} f(q),
$$

where $f(q)$ is a poorly constrained distribution over the initial mass ratio of binary components.

One usually assumes a mass ratio distribution law in the form $f(q) \sim q^{-\alpha_{q}}$ where $\alpha_{q}$ is a parameter, also derived observationally, see Section 1. Another often used form of the $q$-distribution was suggested by Kuiper [391]:

$$
f(q)=2 /(1+q)^{2} .
$$

The range of $A$ is $10 \leq A / R_{\odot} \leq 10^{6}$. In deriving the above Eq. (62), Popova et al. [604] took into account selection effects to convert the "observed" distribution of stars into the true one. An almost flat logarithmic distribution of semimajor axes was also found in [5].

Taking Eq. (62) at face value, assuming $100 \%$ binarity, the mass range of the primary components $M_{1}=0.08 M_{\odot}$ to $100 M_{\odot}$, a flat distribution over semimajor axes (contact at ZAMS) $\leq$ $\log \left(A / R_{\odot}\right) \leq$ (border between close and wide binaries), $f(q)=1$ for close binaries, and $f(q) \propto q^{-2.5}$ for $0.3 \leq q \leq 1$ and $f(q)=2.14$ for $q<0.3$ for wide binaries with $\log \left(A / R_{\odot}\right) \leq 6$, as accepted in Tutukov and Yungelson's BPS code IBIS [791], we get SFR $\approx 8 M_{\odot}$ per yr, which is several times higher than modern estimates of the current Galactic SFR. However, if $8 M_{\odot}$ per year is used as a constant average SFR for 13.5 Gyr, we get the right mass of the Galactic disc. Clearly, Eq. (62) is rather approximate, since most of the stellar mass resides in low-mass stars for which IMF, $f(q)$, $f(A)$, binary fraction, etc., are poorly known. However, if we consider only solar chemical composition stars with $M_{1}>0.95 M_{\odot}$ (which can evolve off the main-sequence in the Hubble time), we get, under the "standard" assumptions in the IBIS-code, e.g., a WD formation rate of 0.65 per yr, which is reasonably consistent with observational estimates (see Liebert et al. [420]), the $\mathrm{SN} \mathrm{II} \mathrm{+} \mathrm{SN} \mathrm{Ib/c} \mathrm{rate} \mathrm{about} \mathrm{1.5/100} \mathrm{per} \mathrm{yr,} \mathrm{which} \mathrm{is} \mathrm{consistent} \mathrm{with} \mathrm{the} \mathrm{inferred} \mathrm{Galactic} \mathrm{rate} \mathrm{[86])}$ or with the pulsar formation rate [816]. We also get the "proper" rate of WD+WD mergers with Superchandrasekhar total mass for SN Ia (a few per thousand years).

Constraints from conservative evolution. For this estimate we shall assume that the primary mass should be at least $10 M_{\odot}$. Equation (62) tells us that the formation rate of such binaries is about 1 per 50 years. We shall restrict ourselves by considering only close binaries, in which mass transfer onto the secondary is possible. This narrows the binary separation interval to $10-1000 R_{\odot}$ (see Figure 5$)$; the birth rate of close massive $\left(M_{1}>10 M_{\odot}\right)$ binaries is thus $1 / 50 \times 2 / 5 \mathrm{yr}^{-1}=$ $1 / 125 \mathrm{yr}^{-1}$. The mass ratio $q$ should not be very small to make the formation of the second NS possible. The lower limit for $q$ is derived from the condition that after the first mass transfer stage, during which the mass of the secondary increases, $M_{2}+\Delta M \geq 10 M_{\odot}$. Here $\Delta M=M_{1}-M_{\mathrm{He}}$ 
and the mass of the helium core left after the first mass transfer is $M_{\mathrm{He}} \approx 0.1\left(M_{1} / M_{\odot}\right)^{1.4}$. This yields

$$
m_{2}+\left(m_{1}-0.1 m_{1}^{1.4}\right)>10,
$$

where we used the notation $m=M / M_{\odot}$, or in terms of $q$ :

$$
q \geq 10 / m_{1}+0.1 m_{1}^{0.4}-1 .
$$

An upper limit for the mass ratio is obtained from the requirement that the binary system remains bound after the sudden mass loss in the second supernova explosion. ${ }^{21}$ From Eq. (51) we obtain

$$
\frac{0.1\left[m_{2}+\left(m_{1}-0.1 m_{1}^{1.4}\right)\right]^{1.4}-1.4}{2.8}<1,
$$

or in terms of $q$ :

$$
q \leq 14.4 / m_{1}+0.1 m_{1}^{0.4}-1 .
$$

Inserting $m_{1}=10$ in the above two equations yields the appropriate mass ratio range $0.25<$ $q<0.69$, i.e., $20 \%$ of the binaries for Kuiper's mass-ratio distribution. So we conclude that the birth rate of binaries that can potentially produce a NS binary system is $\lesssim 0.2 \times 1 / 125 \mathrm{yr}^{-1} \simeq$ $1 / 600 \mathrm{yr}^{-1}$.

Of course, this is a very crude upper limit - we have not taken into account the evolution of the binary separation, ignored initial binary eccentricities, non-conservative mass loss, etc. However, it is not easy to treat all these factors without additional knowledge of numerous details and parameters of binary evolution (such as the physical state of the star at the moment of the Roche lobe overflow, the common envelope efficiency, etc.). All these factors should decrease the formation rate of NS binaries. The coalescence rate of compact binaries (which is ultimately of interest to us) will be even smaller - for the compact binary to merge within the Hubble time, the binary separation after the second supernova explosion should be less than $\sim 100 R_{\odot}$ (orbital periods shorter than $\sim 40 \mathrm{~d}$ ) for arbitrary high orbital eccentricity $e$ (see Figure 3). The model-dependent distribution of NS kick velocities provides another strong complication. We also stress that this upper limit was obtained assuming constant Galactic star-formation rate and a normalization of the binary formation by Eq. (62).

Further (semi-)analytical investigations of the parameter space of binaries leading to the formation of coalescing NS binaries are still possible but technically very difficult, and we shall not reproduce them here. The detailed semi-analytical approach to the problem of the formation of NSs in binaries and the evolution of compact binaries has been developed by Tutukov and Yungelson [787, 788].

\subsection{Population synthesis results}

A distinct approach to the analysis of stellar binary evolution is based on the population synthesis method - a Monte Carlo simulation of the evolution of a sample of binaries with different initial parameters. This approach was first applied to model various observational manifestations of magnetized NSs in massive binary systems [377, 378, 147] and generalized to binary systems of arbitrary mass in [428] (The Scenario Machine code). To achieve a sufficient statistical significance, such simulations usually involve a large number of binaries, typically on the order of a million. The total number of stars in the Galaxy is still four orders of magnitude larger, so this approach cannot guarantee that rare stages of the binary evolution will be adequately reproduced. ${ }^{22}$

\footnotetext{
${ }^{21}$ For the first supernova explosion without kick this is always satisfied.

22 Instead of Monte Carlo simulations one may use a sufficiently dense grid in the 3D space of binary parameters and integrate over this grid (see, e.g., [791] and references therein).
} 
Table 6: Examples of the estimates for Galactic merger rates of relativistic binaries calculated under different assumptions on the parameters entering population synthesis.

\begin{tabular}{lrrrr}
\hline Authors & Ref. & $\begin{array}{r}\mathrm{NS}+\mathrm{NS} \\
{\left[\mathrm{yr}^{-1}\right]}\end{array}$ & $\begin{array}{r}\mathrm{NS}+\mathrm{BH} \\
{\left[\mathrm{yr}^{-1}\right]}\end{array}$ & $\begin{array}{r}\mathrm{BH}+\mathrm{BH} \\
{\left[\mathrm{yr}^{-1}\right]}\end{array}$ \\
\hline Tutukov and Yungelson (1993) & {$[788]$} & $3 \times 10^{-4}$ & $2 \times 10^{-5}$ & $1 \times 10^{-6}$ \\
Lipunov et al. (1997) & {$[429]$} & $3 \times 10^{-5}$ & $2 \times 10^{-6}$ & $3 \times 10^{-7}$ \\
Portegies Zwart and Yungelson (1998) & {$[607]$} & $2 \times 10^{-5}$ & $10^{-6}$ & \\
Nelemans et al. (2001) & {$[518]$} & $2 \times 10^{-5}$ & $4 \times 10^{-6}$ & \\
Voss and Tauris (2003) & {$[815]$} & $2 \times 10^{-6}$ & $6 \times 10^{-7}$ & $10^{-5}$ \\
De Donder and Vanbeveren (2004) & {$[134]$} & $3 \times 10^{-3}-10^{-5}$ & $3 \times 10^{-5}$ & 0 \\
O'Shaughnessy et al. (2005) & {$[547]$} & $7 \times 10^{-6}$ & $1 \times 10^{-6}$ & $1 \times 10^{-6}$ \\
de Freitas Pacheco et al. (2006) & {$[136]$} & $2 \times 10^{-5}$ & & $(0.05-29.7)$ \\
Dominik et al. (2012) & {$[157]$} & $(0.4-77.4)$ & $(0.002-10.6)$ & $\times 10^{-6}$ \\
& & $\times 10^{-6}$ & $\times 10^{-6}$ & 0 \\
Mennekens and Vanbeveren (2013) & {$[479]$} & $10^{-7}-10^{-5}$ & $10^{-6}-10^{-5}$ & \\
\hline
\end{tabular}

Presently, there are several population synthesis codes used for massive binary system studies, which take into account with different degrees of completeness various aspects of stellar binary evolution (e.g., the codes by Portegies Zwart, Nelemans et al. [607, 872], Bethe and Brown [44], Hurley, Tout, and Pols [294], Belczynski et al. [35], Yungelson and Tutukov [791], De Donder and Vanbeveren [133]). A review of applications of the population synthesis method to various types of astrophysical sources and further references can be found in [602, 869]. Some results of populationsynthesis calculations of compact-binary mergers carried out by different groups are presented in Table 6.

Actually, the authors of the studies mentioned in Table 6 make their simulations for a range of parameters. We list in the table the rates for the models the authors themselves consider as "standard" or "preferred" or "most probable", calculated for solar metallicity (or give the ranges). Generally, for the NS + NS merger rate Table 6 shows the scatter within a factor $\sim 4$, which may be considered quite reasonable, having in mind the uncertainties in input parameters. There are several outliers, [788], [815], and [479]. The high rate in [788] is due to the assumption that kicks to nascent neutron stars are absent. The low rate in [815] is due to the fact that these authors apply in the common envelope equation an evolutionary-stage-dependent structural constant $\lambda$. Their range for $\lambda$ is $0.006-0.4$, to be compared with the "standard" $\lambda=0.5$ applied in most of the other studies. Low $\lambda$ favors mergers in the first critical lobe overflow episode and later mergers of the first-born neutron stars with their non-relativistic companions ${ }^{23}$. A considerable scatter in the rates of mergers of systems with $\mathrm{BH}$ companions is due, mainly, to uncertainties in stellar wind mass loss for the most massive stars. For instance, the implementation of winds in the code used in $[607,518]$ resulted in the absence of merging $\mathrm{BH}+\mathrm{BH}$ systems, while a rather low $\dot{M}$ assumed in [815] produced a high merger rate of $\mathrm{BH}+\mathrm{BH}$ systems. We note an extreme scatter of the estimates of the merger rate of NS + NS binaries in [157]: the lowest estimate is obtained assuming very tightly bound envelopes of stars (with parameter $\lambda=0.01$ ), while the upper estimate - assuming completely mass-conservative evolution. The results of the Brussels group [134, 479] differ from STARTRACK-code results [157] and other codes in predicting an insignificant BH-BH merging rate. This is basically due to assumed enhanced mass loss in the red supergiant stage

\footnotetext{
${ }^{23}$ Note that similar low values of $\lambda$ for 20 to $50 M_{\odot}$ stars were obtained also in [598]. If confirmed, these results may have major impact on the estimates of merger rates for relativistic binaries.
} 
(RSG) of massive star evolution. In this scenario, unlike, e.g., Voss and Tauris' assumptions [815], the allowance for the enhanced mass loss at the Luminous Blue Variable (LBV) phase of evolution for stars with an initial mass $\gtrsim 30-40 M_{\odot}$ leads to a significant orbital increase and hence the avoidance of the second Roche-lobe overflow and spiral-in process in the common envelope, which completely precludes the formation of close BH binary systems merging within the Hubble time. The Brussels code also takes into account the time evolution of Galactic metallicity enrichment by massive single and binary stars. A more detailed comparison of different population synthesis results of $\mathrm{NS}+\mathrm{NS}, \mathrm{NS}+\mathrm{BH}$ and $\mathrm{BH}+\mathrm{BH}$ formation and merging rates can be found in [2].

A word of caution. It is hardly possible to trace the detailed evolution of each binary, so approximate descriptions of evolutionary tracks of stars, their interaction, effects of supernovae, etc. are invoked. Thus, fundamental uncertainties of stellar evolution mentioned above are complemented by (i) uncertainties of the scenario and (ii) uncertainties in the normalization of the calculations to the real Galaxy (such as the fraction of binaries among all stars, the star formation history, etc.). The intrinsic uncertainties in the population synthesis results (for example, in the computed event rates of binary mergers etc.) are in the best case not less than $\simeq(2-3)$. This should always be borne in mind when using population synthesis calculations. However, we emphasize again the fact that the NS binary merger rate, as inferred from pulsar binary statistics with account for pulsar binary observations [77, 336, 363], is very close to the population syntheses estimates assuming NS kicks of about $(250-300) \mathrm{km} \mathrm{s}^{-1}$. 


\section{Detection Rates}

From the point of view of detection, a gravitational-wave signal from merging close binaries is characterized by the signal-to-noise ratio $S / N$, which depends on the binary masses, the distance to the binary, the frequency, and the detector's noise characteristics. A pedagogical derivation of the signal-to-noise ratio and its discussion for different detectors is given, for example, in Section 8 of the review [252].

Coalescing binaries emit gravitational wave signals with a well known time-dependence (waveform) $h(t)$ (see Section 3.1 above). This allows one to use the technique of matched filtering [758]. The signal-to-noise ratio $S / N$ for a particular detector, which is characterized by the noise spectral density $S_{n}(f)\left[\mathrm{Hz}^{-1 / 2}\right]$ or the dimensionless noise rms amplitude $h_{\mathrm{rms}}$ at a given frequency $f$, depends mostly on the "chirp" mass of the binary system $\mathcal{M}=\left(M_{1}+M_{2}\right)^{-1 / 5}\left(M_{1} M_{2}\right)^{3 / 5}=\mu^{3 / 5} M^{2 / 5}$ (here $\mu=M_{1} M_{2} / M$ is the reduced mass and $M=M_{1}+M_{2}$ is the total mass of the system) and its (luminosity) distance $r: S / N \propto \mathcal{M}^{5 / 6} / r$ [758, 200]. For a given type of coalescing binary (NS $+\mathrm{NS}$, $\mathrm{NS}+\mathrm{BH}$ or $\mathrm{BH}+\mathrm{BH})$, the signal-to-noise ratio will also depend on the frequency of the innermost stable circular orbit $f_{\text {ISCO }} \sim 1 / M$, as well as on the orientation of the binary with respect to the given detector and its angular sensitivity (see, e.g., Section 8 in [252] and [673] for more detail). Therefore, from the point of view of detection of the specific type of coalescing binaries at a prerequisite signal-to-noise ratio level, it is useful to determine the detector's maximum (or "horizon") distance $D_{\text {hor }}$, which is calculated for an optimally oriented ("ideal") coalescing binary with a given chirp mass $\mathcal{M}$. For a secure detection, the $S / N$ ratio is usually raised up to $7-8$ to avoid false alarms over a period of a year (assuming Gaussian noise). ${ }^{24}$ This requirement determines the maximum distance from which an event can be detected by a given interferometer [126, 2]. The horizon distance $D_{\text {hor }}$ of LIGO I/VIRGO (advanced LIGO, expected) interferometers for relativistic binary inspirals with account of the actual noise curve attained in the S5 LIGO scientific run are given in [2]: $33(445) \mathrm{Mpc}$ for $\mathrm{NS}+\mathrm{NS}\left(1.4 M_{\odot}+1.4 M_{\odot}, \mathcal{M}_{\mathrm{NS}}=1.22 M_{\odot}\right), 70(927) \mathrm{Mpc}$ for $\mathrm{NS}+\mathrm{BH}\left(1.4 M_{\odot}+10 M_{\odot}\right)$, and $161(2187) \mathrm{Mpc}$ for $\mathrm{BH}+\mathrm{BH}\left(10 M_{\odot}+10 M_{\odot}, \mathcal{M}_{\mathrm{BH}}=8.7 M_{\odot}\right)$. The distances increase for a network of detectors.

It is worth noting that the dependence of the $S / N$ for different types of coalescing binaries on $f_{\text {ISCO }}$ changes rather slightly (to within 10\%) as $S / N \propto \mathcal{M}^{5 / 6}$, so, for example, the ratio of $\mathrm{BH}$ and NS detection horizons scales as $S / N$, i.e., $D_{\mathrm{hor}, \mathrm{BH}} / D_{\text {hor, } \mathrm{NS}}=\left(\mathcal{M}_{\mathrm{BH}} / \mathcal{M}_{\mathrm{NS}}\right)^{5 / 6} \approx 5.14$. This allows us to estimate the relative detection ratio for different types of coalescing binaries by a given detector (or a network of detectors). Indeed, at a fixed level of $S / N$, the detection volume is proportional to $D_{\text {hor }}^{3}$ and therefore it is proportional to $\mathcal{M}^{5 / 2}$. The detection rate $\mathcal{D}$ for binaries of a given class $(\mathrm{NS}+\mathrm{NS}, \mathrm{NS}+\mathrm{BH}$ or $\mathrm{BH}+\mathrm{BH})$ is the product of their coalescence rate $\mathcal{R}_{\mathrm{V}}$ and the detector's horizon volume $\propto \mathcal{M}^{5 / 2}$ for these binaries.

It is seen from Table 6 that the model Galactic rate $\mathcal{R}_{\mathrm{G}}$ of NS + NS coalescences is typically higher than the rate of $\mathrm{NS}+\mathrm{BH}$ and $\mathrm{BH}+\mathrm{BH}$ coalescences. However, the $\mathrm{BH}$ mass is significantly larger than the NS mass. So a binary involving one or two black holes, placed at the same distance as a NS + NS binary, produces a significantly larger amplitude of gravitational waves. With the given sensitivity of the detector (fixed $S / N$ ratio), a $\mathrm{BH}+\mathrm{BH}$ binary can be seen at a greater distance than a NS + NS binary. Hence, the registration volume for such bright binaries is significantly larger than the registration volume for relatively weak binaries. The detection rate of a given detector depends on the interplay between the coalescence rate and the detector's response to the sources of one or another kind.

If we assign some characteristic (mean) chirp mass to different types of NS and BH binary

24 If a network of three detectors, such as two LIGOs and VIRGO, runs simultaneously, the $S / N$ ratio in an individual detector should be $>7 / \sqrt{3} \approx 4$. 
systems, the expected ratio of their detection rates by a given detector is

$$
\frac{\mathcal{D}_{\mathrm{BH}}}{\mathcal{D}_{\mathrm{NS}}}=\frac{\mathcal{R}_{\mathrm{BH}}}{\mathcal{R}_{\mathrm{NS}}}\left(\frac{\mathcal{M}_{\mathrm{BH}}}{\mathcal{M}_{\mathrm{NS}}}\right)^{5 / 2}
$$

where $\mathcal{D}_{\mathrm{BH}}$ and $\mathcal{D}_{\mathrm{NS}}$ refer to $\mathrm{BH}+\mathrm{BH}$ and NS + NS pairs, respectively. Taking $\mathcal{M}_{\mathrm{BH}}=8.7 M_{\odot}$ (for $\left.10 M_{\odot}+10 M_{\odot}\right)$ and $\mathcal{M}_{\mathrm{NS}}=1.22 M_{\odot}\left(\right.$ for $\left.1.4 M_{\odot}+1.4 M_{\odot}\right)$, Eq. (65) yields

$$
\frac{\mathcal{D}_{\mathrm{BH}}}{\mathcal{D}_{\mathrm{NS}}} \approx 140 \frac{\mathcal{R}_{\mathrm{BH}}}{\mathcal{R}_{\mathrm{NS}}} .
$$

As $\frac{\mathcal{R}_{\mathrm{BH}}}{\mathcal{R}_{\mathrm{NS}}}$ is typically $0.1-0.01$ (see Table 6), this relation suggests that the registration rate of $\mathrm{BH}$ mergers can be higher than that of NS mergers. Of course, this estimate is very rough, but it can serve as an indication of what one can expect from detailed calculations. We stress that the effect of an enhanced detection rate of $\mathrm{BH}$ binaries is independent of the desired $S / N$ and other characteristics of the detector; it was discussed, for example, in [788, 429, 252, 157].

Unlike the ratio of the detection rates, the expected value of the detection rate of a specific type of compact coalescing binaries by a given detector (network of detectors) requires detailed evolutionary calculations and the knowledge of the actual detector's noise curve, as discussed. To calculate a realistic detection rate of binary mergers the distribution of galaxies should be taken into account within the volume bounded by the detector's horizon (see, for example, the earlier attempt to take into account only bright galaxies from Tully's catalog of nearby galaxies in [425], and the use of the LEDA database of galaxies to estimate the detection rate of supernovae explosions [27]). In this context, a complete study of galaxies within $100 \mathrm{Mpc}$ was done by Kopparapu et al. [376]. Based on their results, Abadie et al. [2] derived the approximate formula for the number of the equivalent Milky-Way-type galaxies within large volumes, which is applicable for distances $\gtrsim 30 \mathrm{Mpc}:$

$$
N_{G}\left(D_{\text {hor }}\right)=\frac{4 \pi}{3}\left(\frac{D_{\text {hor }}}{\mathrm{Mpc}}\right)^{3}(0.0116)(2.26)^{-3} .
$$

Here the factor 0.0116 is the local density of the equivalent Milky-Way-type galaxies derived in [376], and the factor 2.26 takes into account the reduction in the detector's horizon value when averaging over all sky locations and orientations of the binaries. Then the expected detection rate becomes $\mathcal{D}\left(D_{\text {hor }}\right)=\mathcal{R}_{G} \times N_{G}\left(D_{\text {hor }}\right)$.

However, not only the mass and type of a given galaxy, but also the star formation rate and, better, the history of the star formation rate in that galaxy are needed to estimate the expected detection rate $\mathcal{D}$ (since the coalescence rate of compact binaries in the galaxies strongly evolves with time [432, 479, 158]).

So to assess the merger rate from a large volume based on galactic values, the best one can do at present appears to be using formulas like Eq. (67) or (5), given in Section 2.2. However, this adds another factor two of uncertainty to the estimates. Clearly, a more accurate treatment of the transition from galactic rates to larger volumes with an account of the galaxy distribution is very desirable.

To conclude, we will briefly comment on the possible electromagnetic counterparts of compact binary coalescences. It is an important issue, since the localization error boxes of NS + NS coalescences by GW detectors network only are expected to be, in the best case, about several square degrees (see the detailed analysis in [649], as well as [1] for a discussion of the likely evolution of sensitivity and sky localization of sources for the advanced detectors), which is still large for precise astronomical identification. Any associated electromagnetic signal can greatly help to pinpoint the source. NS binary mergings are the most likely progenitors of short gamma-ray bursts $([500,204]$ and references therein). Indeed, recently, a short-hard GRB 130603B was found to be followed by a 
rapidly fading IR afterglow [43], which is most likely due to a 'macronova' or 'kilonova' produced by decaying radioactive heavy elements expelled during a NS binary merging [416, 658, 483, 290, 253]. Detection of electromagnetic counterparts to GW signals from coalescing binaries is an essential part of the strategy of the forthcoming advanced LIGO/VIRGO observations [706]. Different aspects of this multi-messenger GW astronomy are further discussed in papers [562, 530, 343, 1, 466], etc. 


\section{Short-Period Binaries with White-Dwarf Components}

Binary systems with white-dwarf components that are interesting for general relativity and cosmology come in several flavors:

- Detached white-dwarf binaries or "double degenerates" (DDs, we shall use both terms as synonyms below).

- Cataclysmic variables (CVs) - a class of variable semidetached binaries containing a white dwarf and a companion star that is usually a red dwarf or a slightly evolved star, a subgiant.

- A subclass of the former systems in which the Roche lobe is filled by another white dwarf or low-mass partially degenerate helium star (AM CVn-type stars or "interacting doubledegenerates", IDDs). They appear to be important "verification sources" for planned spacebased low-frequency GW detectors.

- Detached systems with a white dwarf accompanied by a low-mass non-degenerate helium star (sd + WD systems).

- Ultracompact X-ray binaries (UCXBs) containing a NS and a Roche lobe overflowing WD or low-mass partially degenerate helium star.

As Figure 2 shows, compact stellar binaries emit gravitational waves within the sensitivity limits for space-based detectors if their orbital periods range from $\sim 20 \mathrm{~s}$ to $\sim 20000 \mathrm{~s}$. This means that, in principle, gravitational-wave radiation may play a pivotal role in the evolution of all AM CVn-stars, UCXBs, a considerable fraction of CVs, and some DD and sd + WD systems and they would be observable in GWs if detectors would be sensitive enough and confusion noise absent.

Though general relativity (GR) predicted that stellar binaries would be a source of gravitational waves as early as in the 1920 s, this prediction became a matter of actual interest only with the discovery of the cataclysmic variable WZ Sge with orbital period $P_{\text {orb }} \approx 81.5$ min by Kraft, Mathews, and Greenstein in 1962 [384], who immediately recognized the significance of shortperiod stellar binaries as testbeds for gravitational wave physics. Another impetus to the study of binaries as sources of gravitational wave radiation (GWR) was imparted by the discovery of the ultra-short period variability of a faint blue star HZ $29=\mathrm{AM}$ CVn $\left(P_{\text {orb }} \approx 18\right.$ min) by Smak in 1967 [719]. Smak [719] and Paczyński [551] speculated that the latter system is a close pair of white dwarfs, without specifying whether it is detached or semidetached. Faulkner et al. [195] inferred the status of AM CVn as a "semidetached white-dwarf-binary" nova. AM CVn was later classified as a cataclysmic variable after flickering typical for CVs was found for AM CVn by Warner and Robinson $[822]^{25}$ and it became the prototype for a subclass of binaries. ${ }^{26}$

An evident milestone in GW studies was the discovery of a pulsar binary by Hulse and Taylor $[757,292]$ and the determination of the derivative of its $P_{\text {orb }}$, consistent with GR predictions [832]. Equally significant is the recent discovery of a $P_{\text {orb }}=12.75$ min detached eclipsing white dwarf binary SDSS J065133.338+284423.37 (J0651, for simplicity) by Kilic, Brown et al. [359, 73]. The measured change of the orbital period of J0651 over 13 months of observations is $(-9.8 \pm 2.8) \times 10^{-12} \mathrm{~s} \mathrm{~s}^{-1}$, which is consistent, within $3 \sigma$ errors, with expectations from $\mathrm{GR}-(-8.2 \pm 1.7) \times 10^{-12} \mathrm{~s} \mathrm{~s}^{-1}$ [277]. The strain amplitude of gravitational waves from this object at a frequency of $\sim 2.6 \mathrm{mHz}$ should be $1.2 \times 10^{-22} \mathrm{~Hz}^{-1 / 2}$, which is about 10000 times as high

\footnotetext{
25 Flickering is a fast intrinsic brightness scintillation occurring on time scales from seconds to minutes with amplitudes of 0.01-1 mag and suggesting an ongoing mass transfer.

26 Remarkably, a clear-cut confirmation of the binary nature of AM CVn and the determination of its true period awaited for almost 25 years [513].
} 
as that from the Hulse-Taylor binary pulsar. It is currently the second most powerful GW source known and it is expected to be discovered in the first weeks of operation of eLISA. ${ }^{27}$

The origin of all above-mentioned classes of short-period binaries was understood after the notion of common envelopes and the formalism for their treatment were suggested in the 1970s (see Section 3.6). A spiral-in of components in common envelopes allowed to explain how white dwarfs - former cores of highly evolved stars with radii of $\sim 100 R_{\odot}$ - may acquire companions separated by $\sim R_{\odot}$ only. However, we recall that most studies of the formation of compact objects through common envelopes are based on a simple formalism of comparison of binding energy of the envelope with the orbital energy of the binary, supposed to be the sole source of energy for the loss of the envelope, as was discussed in Section 3.6.

Although we mentioned that some currently-accepted features of binary evolution may be subject to certain revisions in the future, one may expect that, for example, changes in the stability criteria for mass exchange will influence mainly the parameter space $\left(M_{1}, M_{2}, a_{0}\right)$ of progenitors of particular populations of stellar binaries, but not the evolutionary scenarios for their formation. If the objects of a specific class may form via different channels, the relative "weight" of the latter may change. While awaiting for detailed evolutionary calculations of different cases of mass exchange with "new" physics, ${ }^{28}$ we present the currently-accepted scheme of the evolution of binaries leading to the formation of compact binary systems with WD components.

For stars with radiative envelopes, to the first approximation, the mass transfer from $M_{1}$ to $M_{2}$ is stable for binary mass ratios $q=M_{2} / M_{1} \lesssim 1.2$; for $1.2 \lesssim q \lesssim 2$ it proceeds in the thermal time scale of the donor, $t_{K H}=G M^{2} / R L$; for $q \gtrsim 2$ it proceeds in the dynamical time scale $t_{d}=\sqrt{R^{3} / G M}$. The mass loss occurs in the dynamical time scale, $\dot{M} \sim M / t_{d}$, if the donor has a deep convective envelope or if it is degenerate and conditions for stable mass exchange are not satisfied. However, note that in the case of AGB stars, the stage when the photometric (i.e., measured at the optical depth $\tau=2 / 3$ ) stellar radius becomes equal to the Roche lobe radius, is preceded by RLOF by atmospheric layers of the star, and the dynamic stage of mass loss may be preceded by a quite long stage of stable mass loss from the radiative atmosphere of the donor [555, 594, 490]. It is currently commonly accepted, despite the lack of firm observational proof, that the distribution of binaries over $q$ is even or rises to small $q$ (see Section 5.1). Since the accretion rate is limited either by the rate that corresponds to the thermal time scale of the accretor or its Eddington accretion rate, both of which are typically lower than the mass-loss rate by the donor, the overwhelming majority $(\sim 90 \%)$ of close binaries pass in their evolution through one to four common envelope stages.

An "initial donor mass - donor radius at RLOF" diagram showing descendants of stars after mass-loss in close binaries is presented above in Figure 5. We remind here that solar metallicity stars with $M \lesssim 0.95 M_{\odot}$ do not evolve past the core-hydrogen burning stage in the Hubble time.

\subsection{Formation of compact binaries with white dwarfs}

A flowchart schematically presenting the typical scenarios for formation of low-mass compact binaries with WD components and some endpoints of evolution is shown in Figure 9. Evolution of low and intermediate-mass close binaries $\left(M_{1} \leq(8-10) M_{\odot}\right)$ is generally much more complex than the evolution of more massive binaries (Figure 7). For this reason, not all possible scenarios are plotted, but only the most probable routes to SNe Ia and to systems that may emit potentially detectable gravitational waves. For simplicity, we consider only the most general case when the first RLOF results in the formation of a common envelope.

\footnotetext{
27 It is expected that recently launched astrometric space mission GAIA will detect about 200 eclipsing double degenerates [511].

28 The advent of new, fast and stable stellar evolutionary codes like MESA [574, 575], http://mesa.sourceforge. net will, hopefully, facilitate achieving this aim in the near future.
} 


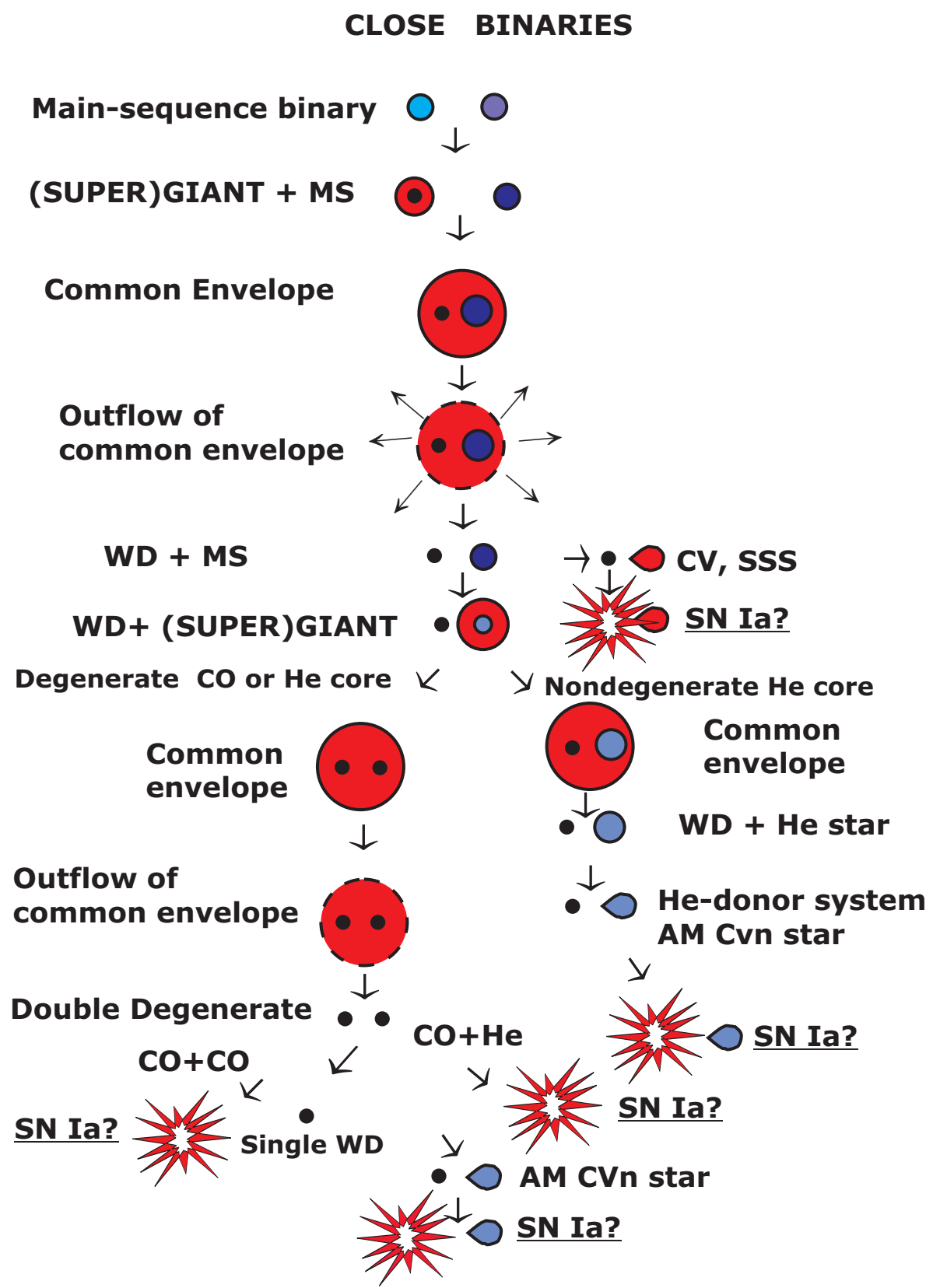

Figure 9: Formation of close dwarf binaries and their descendants (scale and color-coding are arbitrary). 
The overwhelming majority of stars in binaries fill their Roche lobes when they have He- or CO-cores, i.e., in cases B or $\mathbf{C}$ of mass exchange (Section 3.2). As noted in Section 3.6, the models of common envelopes are, in fact, absent. It is usually assumed that it proceeds in a dynamic or thermal time scale and is definitely so short that its duration can be neglected compared to other evolutionary stages.

\subsubsection{Post-common envelope binaries}

We recall, for convenience, that in stars with solar metallicity with a ZAMS mass below $(2.3-2.8) M_{\odot}$, helium cores are degenerate, and if these stars overflow the Roche lobe prior to He core ignition, they produce $M \lesssim 0.47 M_{\odot}$ helium white dwarfs. Binaries with non-degenerate He-core donors $\left(M \gtrsim(2.3-2.8) M_{\odot}\right)$ first form a close He-star + MS-star pair that can be observed as a subdwarf ( $\mathrm{sdB}$ or $\mathrm{sdO})$ star with a MS companion [786]. The minimum mass of He-burning stars is close to $0.33 M_{\odot}$ [301]. The binary hypothesis for the origin of hot subdwarf stars was first suggested by Mengel et al. [478], but it envisioned a stable Roche lobe overflow. Apparently, there exist populations of close $\mathrm{sdB} / \mathrm{sdO}$ stars formed via common envelopes and of wide systems with A-F type companions to subdwarfs, which may be post-stable-mass-exchange stars; similarly, the merger products and genuinely single stars can be found among sdB/sdO stars. For the overview of properties of $\mathrm{sdB} / \mathrm{sdO}$ stellar binaries, models of the population and current state of the problem of the origin of these stars see, e.g., [667, 251, 668, 268, 267, 7, 879, 205, 273, 232, 510, 245, 227, 128, 233]. When a He-star with mass $\lesssim 2.3 M_{\odot}$ completes its evolution, a pair harboring a CO white dwarf and an MS-star appears. A large number of post-common envelope binaries or "WD + MS stars" is known (see, e.g., SDSS-sample and its analysis in [507, 887]). The most recent population synthesis model of this class of binaries was published by Toonen and Nelemans [765]. Of course, the WD + MS population is dominated by systems with low-mass MS-stars. Population synthesis studies suggest that about $1 / 3$ of them never evolve further in the Hubble time (e.g., [869]), in a reasonable agreement with above-mentioned observations.

\subsubsection{Cataclysmic variables}

If after the first common-envelope stage the orbital separation of the binary $a \simeq$ several $R_{\odot}$ and the WD has a low-mass $\left(\lesssim 1.4 M_{\odot}\right)$ main-sequence companion, the latter can overflow its Roche lobe during the hydrogen-burning stage or very shortly after it, because of loss of angular momentum by a magnetically-coupled stellar wind and/or GW radiation, see Sections 3.1.4 and 3.1.5. If the mass ratio of the components allows them to avoid merging in the $\mathrm{CE}(q \lesssim 1.2)$, a cataclysmic variable (CV) can form. Variability of these stars can be due to thermal-viscous instability of accretion disks [546] and/or unstable burning of accreted hydrogen on the WD surface [481, 482, 656, 736, 737, 739]; see Warner's monograph [821] for a comprehensive review of CVs in general, [409] for a review of the disk instability model and, e.g., [559, 296, 143, 175, 694, 613, 770, 859, 695, 535, 701, 696, $587,588,303,242,843,307]$ for dependence of hydrogen burning regimes on the WD surface and characteristics of outbursts, depending on WD masses, their chemical composition (He, CO, $\mathrm{ONe}$ ), temperature and accretion rate. Outbursts produced by thermonuclear burning of accreted hydrogen are identified with novae [736, 737] and are able to explain their different classes, see, e.g., [859]. As an example, we present in Figure 10 the dependence of limits of different burning regimes on mass and accretion rate for CO WD from Nomoto's paper [535]. The stable burning limits found in this paper, by stability analysis of a steady-burning WD, within factor $\simeq 2$ agree with those obtained in a similar way, e.g., by Shen and Bildsten [696] and with the results of time-dependent calculations by Wolf et al. [843] (see Figure 9 in the latter paper.)

Binaries with WDs and similar systems with subgiants $\left(M \lesssim 2 M_{\odot}\right)$ that steadily burn accreted hydrogen are usually identified with supersoft X-ray sources (SSS) [800]. Note, however, that the actual fraction of the stable burning stage when these stars can be observed as SSS is still a matter 


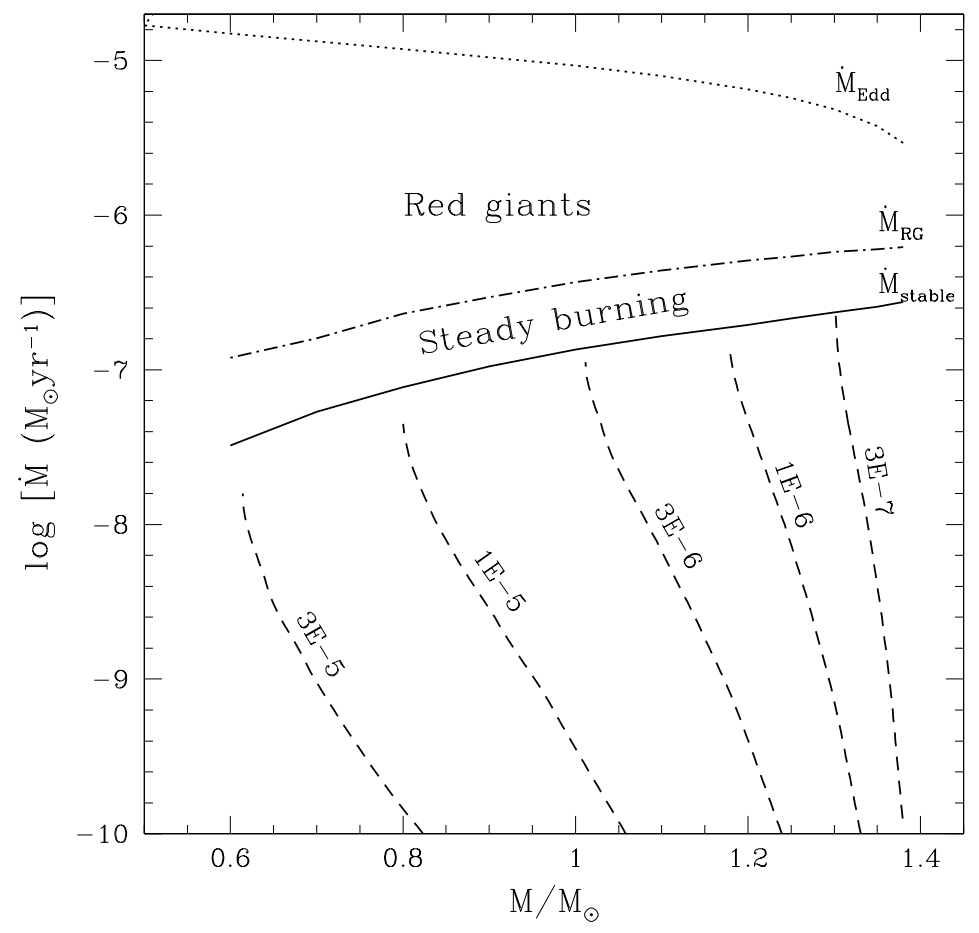

Figure 10: Limits of different burning regimes of accreted hydrogen onto a CO WD as a function of mass of the WD and accretion rate $\dot{M}$ [535]. If $\dot{M}_{\text {stable }} \lesssim \dot{M} \lesssim \dot{M}_{\mathrm{RG}}$, hydrogen burns steadily. If $\dot{M} \lesssim \dot{M}_{\text {stable }}, \mathrm{H}$ burning shells are thermally unstable; with decrease of $\dot{M}$ the strength of flashes increases. For $\dot{M} \gtrsim \dot{M}_{\mathrm{RG}}$, hydrogen burns steadily but the excess of unburnt matter forms an extended red-giant-sized envelope. In the latter case white dwarfs may lose matter by wind or due to Roche lobe overflow. A dotted line shows the Eddington accretion rate $\dot{M}_{\mathrm{Edd}}$ as a function of $M_{\mathrm{WD}}$. The dashed lines are the loci of the hydrogen envelope mass at hydrogen ignition. Image reproduced with permission from [535], copyright by AAS.

of debate, see $[259,528]$. Post-novae in the stage of residual hydrogen burning can also be observed as SSS [773, 276, 524]. In these systems, the duration of the SSS stage is debated as well, see, e.g., [843]. A review of SSS may be found, e.g., in [330]; the population synthesis models for SSS where computed, for instance, in [625, 329, 875, 871].

An accreting CO WD in a binary system can accumulate enough mass to explode as a SN Ia if hydrogen burns stably or in mild flashes. This is the "single-degenerate" (SD) scenario for SNe Ia, originally suggested by Schatzman as early as in 1963 [681] and "rediscovered" and elaborated numerically 10 years later by Whelan and Iben [836]. We shall consider these possible progenitors of SNe Ia in more detail below (Section 7.3).

If the WD belongs to the ONe-variety, it may experience an AIC into a neutron star due to electron captures on $\mathrm{Ne}$ and $\mathrm{Mg}$, and a low-mass X-ray binary may be formed [83].

The final stages of the evolution of CVs are not clear (and, in fact, poorly investigated). It was hypothesized [659] that, when the donor mass decreases below several hundredths of $M_{\odot}$, the disk-orbit tidal interaction becomes inefficient and the disk turns into a sink of orbital momentum. More orbital momentum is drained from the orbit than returned back, the mass loss rate increases and the donor can be tidally disrupted. 
As noted by G. Dubus (private communication) at $q \lesssim 0.02$, which may be attained in the Hubble time, the conventional picture of mass exchange may become invalid: the circularization radius (the minimum radius of the accretion disk defined by the specific angular momentum of the matter at the $L_{1}$ point and its interaction with the donor) exceeds the outer radius of the disk (determined by tidal effects $[554,567,809]$ ). The resonance effects come into play and gaps may appear in the outer disk, since orbital resonances confine the motion of disk particles to certain radius ranges, similar to the formation of gaps in the asteroid belt and Saturn rings. It is not clear how the disk behaves then and how it interacts with still inflowing matter.

\subsection{White-dwarf binaries}

The second common envelope stage may happen when the giant companion to the WD overfills its Roche lobe. Before proceeding to a discussion of later stages, we note the following. Usually, this second common envelope is thought to result, based merely on the final separation of the components as given by Eqs. (56) and (59) - in a merger, if at the end of common envelope event the radius of one of components becomes larger than its Roche-lobe radius, or in the formation of a detached system otherwise. However, as was speculated by Livio and Riess [437] after the first detection of SN Ia with hydrogen in the spectrum (SN 2002ic [264]), there is a very small probability $(\lesssim 1 \%)$ that WD merges with the core of an AGB star when part of the AGB envelope (common envelope) is still not ejected. These considerations are corroborated by results of 1D [313, 341] and 3-D simulations, [571] which show that only a fraction of the common envelope is ejected in the dynamical time scale, and the rest of the matter remains in the vicinity of the close pair formed by the core of the AGB star and the spiraling-in WD. As mentioned in Section 3.6, interaction with this matter may result in further shrinking of the system and ultimate merger of the components. If both cores are carbon-oxygen and the total mass of the merger product exceeds $M_{\mathrm{Ch}}$, it may explode as a SN Ia; see brief discussion of this "core-degenerate" [341] SNe Ia scenario in Section 7.3.

If the system avoids merging inside the $\mathrm{CE}$ and the donor had a degenerate core, a close WD binary or DD is formed (the left branch of the scenario shown in Figure 9). In Figure 11 we show an indicative plot of possible combinations of components in double-degenerate systems [461] (it is assumed that the bulk chemical composition of WD is uniquely related to its mass).

In Figure 12 we compare the most recent simulated distribution of the total masses of WD binaries vs. their $P_{\text {orb }}$ with the distribution of $M_{\text {tot }}$ for 46 observed WD binaries known at the time of writing of the quoted study [766]. The limiting stellar magnitude was assumed to be $V_{\text {lim }}=21$. It is difficult to judge the agreement with observations because the sample of observed WDs is quite random, but the "naked eye" estimate suggests that the model that uses $\gamma$-formalism fits observations better than the model that uses $\alpha$-formalism. 


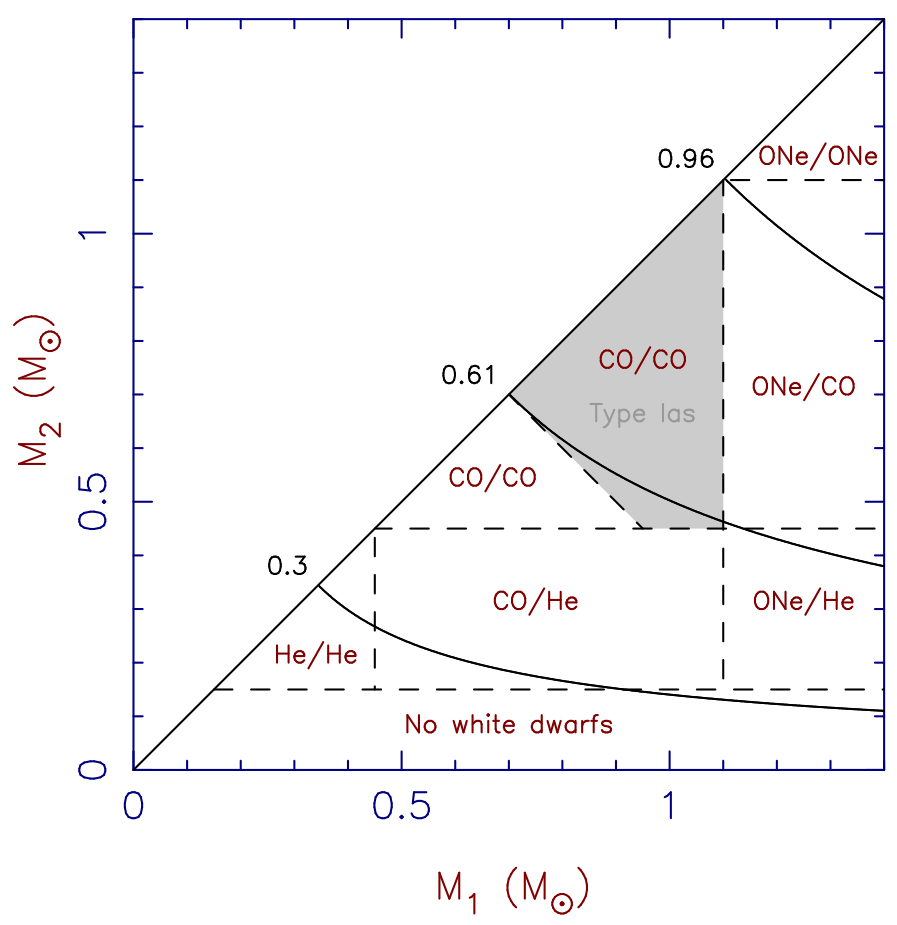

Figure 11: Possible combinations of masses and chemical compositions of components in a close WD binary [461]. Solid curves are lines of constant chirp mass (see Sections 3.1.2 and 10). Image reproduced with permission from [461], copyright by IOP.

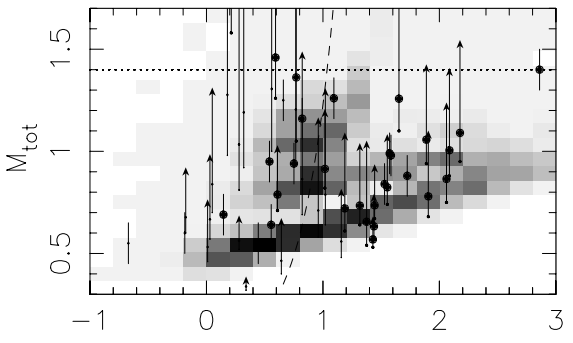

$\log P(h r)$

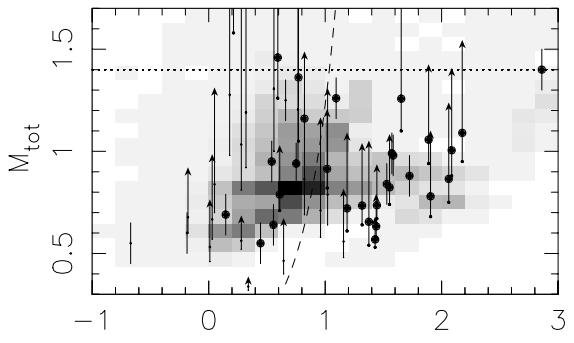

$\log P(h r)$

Figure 12: The total masses of binaries in the simulated population of WD binaries with $V_{\lim }=21$ as a function of system's orbital period. In the left plot the outcome of the first common envelope stage is described by $\gamma$-formalism, Eq. (59), and in the right plot - by $\alpha$-formalism, Eq. (57). In both plots the latter equation describes both common envelope stages. The grey scale (the same for both plots) corresponds to the density of objects in the linear scale. Observed WD binaries are shown as filled circles (see the original paper for references). The Chandrasekhar mass limit is shown by the dotted line. To the left of the dashed line the systems merge within 13.5 Gyr. Image reproduced with permission from [766], copyright by ESO. 
The fate of DDs is solely determined by GWR. The closest of them may be brought into contact by AML via GWR. For instance, a $(0.6+0.6) M_{\odot}$ WD pair can merge in $10 \mathrm{Gyr}$ if the post-CE separation of the components is about $2 R_{\odot}$. We shall discuss the population of observed detached DDs in Section 8. If one of the DD components fills its Roche lobe, the outcome of the contact depends on the chemical composition of the stars and their masses. There are several possible endpoints: merger leading to a supernova (the left and the right-most branches at the bottom of Figure 9, see Section 7.3), stable mass exchange with the formation of an AM CVn system (as indicated in the figure, see Section 9), direct formation of a single massive WD (the central branch, see Section 7.3.2 and discussion of Figure 18) or the formation of an R CrB type star with an extended helium envelope [825, 306, 885, 480], which will also end its evolution as a single WD (not shown in Figure 9). White dwarfs produced by the merger process may be hidden among single WDs without traces of $\mathrm{H}$ or $\mathrm{He}$, like those with oxygen-rich atmospheres [222].

If the donor has a non-degenerate He-core (ZAMS mass $M_{\text {ZAMS }} \gtrsim(2.3-2.8) M_{\odot}$ ) and the system does not merge, after the second CE-stage a helium star + WD system can arise (the "Nondegenerate He-core" branch of evolution shown to the right in Figure 9). From the point of GW detection, low-mass He-stars $\left(M_{\mathrm{He}} \lesssim 0.8 M_{\odot}\right.$ for $\left.Z=0.02\right)$ can be important. The lifetime of Heremnants of the close binary components can be as long as that of their main-sequence progenitors [301]:

$$
t_{\mathrm{He}} \approx 10^{7.15} m_{\mathrm{He}}^{-3.7} \mathrm{yr}
$$

( $m_{\mathrm{He}}$ is the mass in solar units). These stars expand only slightly at the core-helium burning stage. If the separation of components is sufficiently small $\left(P_{\text {orb }} \lesssim(120-140)\right.$ min $)$, AML via GWR may bring He-star to RLOF, while He is still burning in its core. If $M_{\mathrm{He}} / M_{\mathrm{WD}} \lesssim 2$, a stable mass exchange is possible [674, 819]. A detailed study of the evolution of semidetached low-mass He-stars with WD companions may be found in [870], see also Section 9. These stars overfill their Roche lobes at orbital periods ranging from 16 to $50 \mathrm{~min}$ and first evolve to shorter periods with a typical $\dot{M}$ of several $10^{-8} \mathrm{yr}^{-1}$. The mass loss quenches the central nuclear burning, and the helium star becomes "semi-degenerate". An AM CVn-type system may be formed in this way, see Section 9) for more details. One cannot exclude that a Chandrasekhar mass may be accumulated by the WD in this channel of evolution, but the probability of such a scenario seems to be very low, $\sim 1 \%$ of the inferred galactic rate of SNe Ia [727]. If the He-star completes the core He-burning before RLOF, it becomes a CO-WD. In Figure 9 it "jumps" into the "double degenerate" branch of evolution.

If the mass of the helium remnant-star is $\approx(0.8-2.3) M_{\odot}$, it can hardly overfill the Roche lobe in the core He-burning stage, but after exhaustion of He in the core it expands and may refill the Roche lobe [301]. Mass-loss rates of these "helium-giants" occur in the thermal time scale of their envelopes: $\dot{M} \approx 10^{-6 \pm 1}$ Myr. They may lose several $0.1 M_{\odot}$ and, if companion WD is initially sufficiently massive, the latter may accumulate $M_{\mathrm{Ch}}[860,819]$. If the "massive" He-star completes core He-burning before RLOF or its companion does not explode, the He-star turns into a CO-WD. In Figure 9 it "jumps" into the "double degenerate" branch of evolution.

\subsection{Type Ia supernovae}

The most intriguing possible outcomes of the evolution of compact binaries with WDs are explosions of type Ia SNe. Presently, the most stringent constraints on the nature of the exploding object in SN Ia are placed by the recent SN 2011fe. We reproduce from the paper by Bloom et al. [57] Figure 13 showing the constraints on the parameters of the exploded object in SN 2011fe, which were obtained by excluding certain regions of progenitors space on observational grounds. The blank space in Figure 13 leaves only a compact object (WD or NS) as the progenitor to this SN, but the possible NS phase transition to a quark star is highly unlikely. 


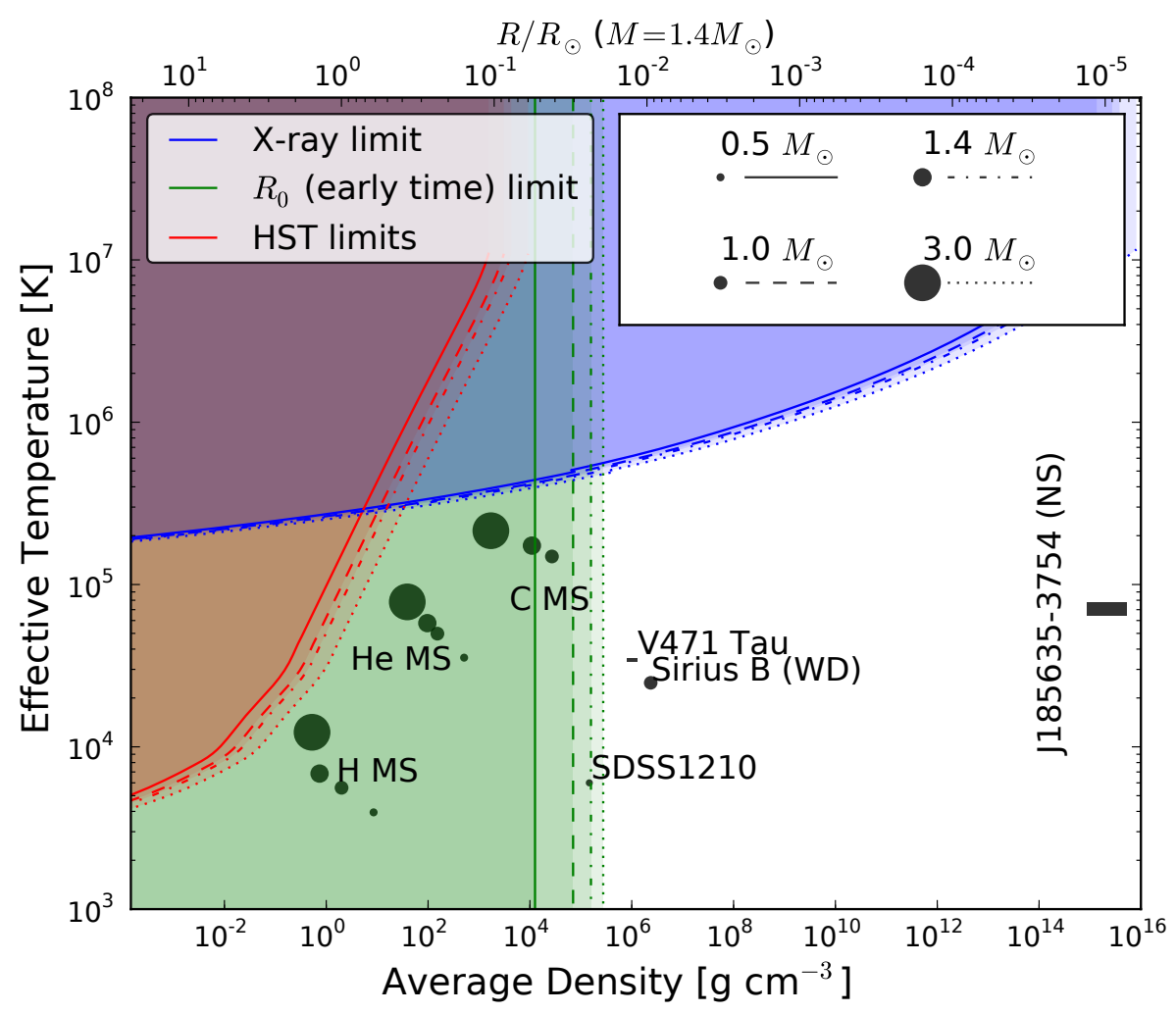

Figure 13: Constraints on mass, effective temperature, radius and average density of the primary star of SN 2011fe. The shaded red region is excluded by non-detection of an optical quiescent counterpart in the Hubble Space Telescope imaging. The shaded green region is excluded from considerations of the non-detection of a shock breakout at early times. The blue region is excluded by the non-detection of a quiescent counterpart in the Chandra X-ray imaging. The location of the $\mathrm{H}, \mathrm{He}$, and $\mathrm{C}$ main-sequence is shown, with the symbol size scaled for different primary masses. Several observed WDs and NSs are shown. The primary radius in units of $R_{\odot}$ is shown for mass $M_{p}=1.4 M_{\odot}$. Image reproduced with permission from [57], copyright by AAS.

The exploding star radius estimate in Figure 13 is derived by assuming that SN 2011fe was discovered about $11 \mathrm{hr}$ after the explosion, as inferred by Nugent et al. [539], from a simple shockbreakout model, which gives a measure of the stellar radius. However, as noted by Piro and Nakar [593], since the early luminosity of SN Ia is powered by radioactive decay of ${ }^{56} \mathrm{Ni}$, if there is no Ni mixed in the outermost layers of the ejecta, there can be a "dark phase" lasting from a few hours to several days, where the only source of emission is radiative cooling of shock-heated gas that is too dim to be detected. Duration of the "dark stage" depends on radial distribution of ${ }^{56} \mathrm{Ni}$. Piro and Nakar suggested that the explosion of SN 2011fe occurred earlier than estimated by Nugent et al., and bounds on the radius of the exploding object are less stringent than those suggested by Bloom et al. However, even a several times larger limit hardly leaves room for any exploding object different from a WD. A comprehensive analysis of data on SN 2011fe obtained until mid-2013 and their implications for the problem of progenitors of SN Ia can be found in the review paper by Chomiuk [107]. Early time observations of SN 2011fe can be explained in the frame of "pulsational-delayed detonations" paradigm for SNe Ia explosions [145].

The theory of stellar evolution should be able to explain the formation of SNe Ia progenitors, 
which fit the basic observational constraints: the explosion energy, the frequency of explosions in the galaxies with different morphology, the observed light curves and spectra, chemical yields, etc.

Until very recently, the most popular paradigms for SNe Ia used the accumulation of the Chandrasekhar mass $M_{\mathrm{Ch}} \approx 1.38 M_{\odot}$ by a CO WD via accretion in semidetached binaries or in a merging WD binary with the total mass exceeding the Chandrasekhar limit. We discuss them below and then proceed to some other ideas actively debated at present.

\subsubsection{Single-degenerate scenario}

The formation of an $M=M_{\mathrm{Ch}}$ WD prone to a thermonuclear explosion seems to be a natural outcome of the accretion of hydrogen in a semidetached binary. The masses of CO WDs are limited by $\approx 1.2 M_{\odot}$ (see Section 1$)$. It is easily envisioned that, if hydrogen burning on the surface of an accreting $\mathrm{CO}$ WD is stable or occurs in mild flashes, as well as the subsequent helium burning, and the WD is not eroded, the matter can be accumulated on the WD surface to increase the WD mass to $M_{\mathrm{Ch}}$. The WD mass growth via the hydrogen burning ("CV, SSS" branch of evolution in Figure 9) is considered to be equivalent to the direct accretion of helium at the rate determined by hydrogen burning [532]. Strictly speaking, the latter assumption is not completely accurate, since H-burning modifies the thermal state of the He-layer that accumulates beneath the H-burning shell, and in the case of the direct accumulation of helium on the WD surface, the WD evolution can be different. The efficiency of matter retention on the WD surface can also differ [94]. However, this issue has not been systematically studied as yet.

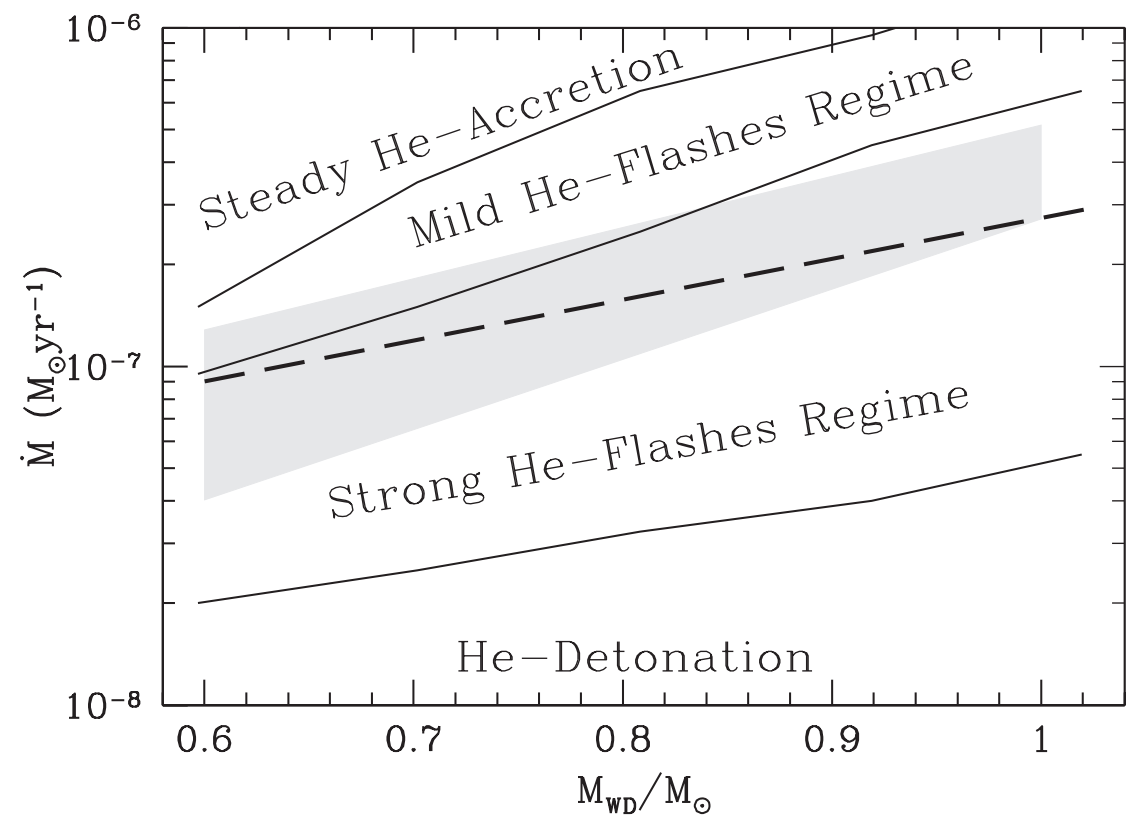

Figure 14: Limits on different burning regimes of helium accreted onto a CO WD as a function of the WD mass and accretion rate [590], Piersanti, Tornambé \& Yungelson (in prep.). Above the "RG Configuration" line, accreted He forms an extended red-giant-like envelope. Below $(3-5) \times 10^{-8} M_{\odot} \mathrm{yr}^{-1}$, accreted He detonates and the mass is lost dynamically. In the strong-flashes regime, He-layer expands and mass is lost due to RLOF and interaction with the companion. Helium accumulation efficiency for this regime is shown in Figure 15, and the critical He masses for WD detonation are shown in Figure 28. Shaded region shows the domain of stable burning of accreted hydrogen. 


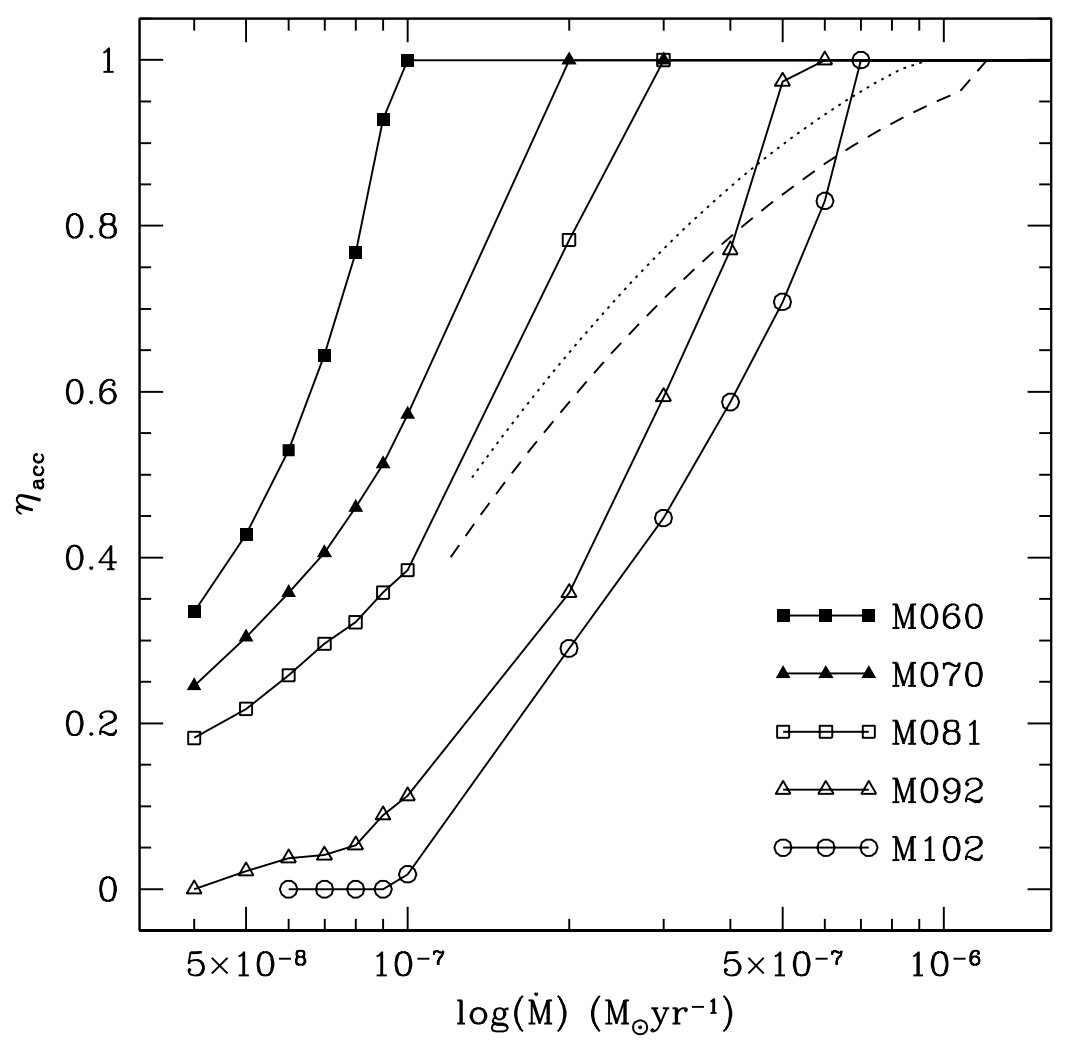

Figure 15: The accumulation efficiency of helium as a function of the accretion rate for CO WD models of $0.6,0.7,0.81,0.92,1.02 M_{\odot}$ (top to bottom), as calculated by Piersanti et al. (in prep.). Dotted and dashed lines represent the accumulation efficiency for CO WDs with initial masses 0.9 and $1.0 M_{\odot}$, respectively, after Kato \& Hachisu [347].

Another version of this scenario, which is sometimes discussed, involves a WD accreting matter from the stellar wind of the companion in wide binary systems (in symbiotic stars). However, the following considerations immediately suggest low efficiency of this channel. In symbiotic binaries, the components evolve independently, hence the typical mass of the WD accretor should be small: $\sim 0.6 M_{\odot}$. The efficiency of accretion from the stellar wind of the optical companion is on the order of $10 \%[62,141]$. Thus, there is little chance to accumulate $M_{\mathrm{Ch}}$, which is also confirmed by numerical simulations [876, 447, 871].

Note that the "single-degenerate"(SD) channel to SN Ia encounters severe problems. Stable burning of hydrogen or burning in mild flashes on the surface of a massive WD may occur within a narrow range of accretion rates $\dot{M} \sim\left(10^{-6}-10^{-7}\right) M_{\odot} \mathrm{yr}^{-1}$ (Figure 14). Mass-loss rates by MS-stars in cataclysmic variables driven by the angular momentum loss via magnetic stellar wind are below several $10^{-8} M_{\odot} \mathrm{yr}^{-1}$ and result in Nova explosions, which limit the growth of $M_{\mathrm{WD}}$ or even cause their erosion $[613,859,175]$. Donors in the Hertzsprung gap or on the early red-giant branch may provide higher $\dot{M}$ if they lose mass in a thermal time scale $\dot{M}_{d, t h}$. Masses of companions should be commensurable to the masses of WD by virtue of the mass-exchange stability conditions. However, the mass loss in these stars in the initial phase of mass exchange proceeds on a time scale that is even shorter than the thermal one [555] and $\dot{M}$ can exceed $\dot{M}_{\mathrm{RG}}$ (Figure 10) by $1-2$ orders 
of magnitude and even be higher than $\dot{M}_{\text {Edd }}$.

It is possible that the precursor of a SD-SN Ia is observed, e.g., in the supersoft X-ray source WX Cen [620]. If for this $P_{\text {orb }}=0.4169615( \pm 22) \mathrm{d}$ system [541], $d P / d t=-5.15 \times 10^{-7} \mathrm{~d} \mathrm{yr}^{-1}$ reflects the real secular decrease of the orbital period, then the donor is more massive than the accretor, and the time scale of mass exchange is thermal. The estimated mass of the WD in this system is $0.9 M_{\odot}$, and there is a chance that the mass of WD will grow up to $M_{\mathrm{Ch}}$. It is sometimes claimed, see, e.g., [349] and references therein, that recurrent novae may also be precursors of SNe Ia. These stars are presumably cataclysmic variables with high-mass $\left(\sim 1.3 M_{\odot}\right)$ WD-components, accreting at rates slightly lower than the $\dot{M}_{\text {stable }}$ (see Figure 10). In this burning regime some mass accumulation is possible, e.g., [613, 859]. However, since it is expected that the accumulation of very little mass is necessary for a massive dwarf to initiate a thermonuclear runaway $\left(\sim 10^{-6} M_{\odot}\right)$, the appropriate "theoretical" novae rate will far exceed the observed rate [729]. Apparently, the mass function of $\mathrm{CO}$ WD in cataclysmic variables is such, that the number of massive WD is vanishingly small.

As a remedy for the deficit of the candidate SD SNe Ia and lack, at that time, of obvious candidates for SN Ia from merging dwarfs, Hachisu et al. [257] suggested that the excess of matter over $\dot{M}_{\mathrm{RG}}$ can be blown out from the system by optically-thick winds, similar to the winds blowing from novae after eruptions, which were introduced into consideration by the same authors [346]. ${ }^{29}$ The efficiency of accretion then becomes $\dot{M}_{d, t h} / \dot{M}_{\mathrm{RG}}$. The angular momentum is lost in the "isotropic reemission" mode, and this also raises the upper limit of the components ratio, thus allowing a stable mass exchange (see the discussion in [257, 873]). The strong wind stops when the mass-loss rate drops below $\dot{M}_{\mathrm{RG}}$. However, even in this regime of steady accretion WD must lose mass via a strong wind, since its surface is hot $\left((1-3) \times 10^{5} \mathrm{~K}\right)$ and, hence, the efficiency of mass capture by the accretor is $<1$. It drops further in the flaring regime, when the expanding envelope of the WD may exceed the corresponding Roche lobe radius [303]. Finally, at $\dot{M}_{\text {accr }} \lesssim$ $10^{-8} M_{\odot} \mathrm{yr}^{-1}$ all accumulated mass is lost in novae explosions [613, 859].

We note parenthetically that it was suggested that WD losing mass in the regime of opticallythick winds (also called "Rapidly Accreting White Dwarfs", RAWD) may be observable as lowluminosity Wolf-Rayet stars, possibly as WR nuclei of planetary nebulae or as V Sge type cataclysmic binaries with numerous emission lines of highly ionized species in their spectra, see [414] and references therein. In principle, RAWDs can exist and can be observed in nearby elliptical galaxies, such as M32 [97]. However, a dedicated survey for RAWDs in the central core of the Small Magellanic Cloud [414] did not reveal a single candidate system. On the other hand, since the appearance of RAWDs has never been modeled in detail, the nondetection of them, in fact, does not constrain the SD model of SNe Ia as yet.

Kato and Hachisu also introduced into the model of SD SNe Ia progenitors the "stripping effect". It assumes that a strong hot wind from the WD irradiates the companion and induces an additional mass loss. The combined effect of the additional mass and angular momentum losses rises further the masses of WD companions, which still allow a stable mass exchange: to about $3 M_{\odot}$ and $6 M_{\odot}$ for MS and red-giant companions, respectively (see $[258,262]$ for the latest versions of the model). However, the "stripping effect" needs a very fine tuning and is hardly feasible.

Further major complication of the SD SNe Ia model is associated with a mismatch of regimes of stable and unstable burning of $\mathrm{H}$ and He, noted by Iben and Tutukov [303]; see the paper by Bours et al. [64] for the latest study of the influence of $\mathrm{H}$ and He retention efficiency upon the rate of SNe Ia with SD-progenitors. In Figure 14 we show the limits of different burning regimes of helium accreted onto a CO WD as a function of the WD mass and accretion rate [590], Piersanti, Yungelson and Tornambé (in prep.) and overplot them by the limits of the domain of stable burning of accreted hydrogen. Figure 14 clearly suggests that, if even some He can be accumulated

29 Note that the existence of these winds has never been rigorously proven by radiative transfer theory. 
via H-burning in the regimes of steady burning or mild flashes, it can be almost entirely lost in strong flashes of He-burning (Figure 15). In fact, the issue of He-accumulation is still not settled. In the latter Figure, we also show for comparison He-accumulation efficiencies computed by Kato \& Hachisu [348] under different assumptions. The most important source of difference is, in our opinion, the ignorance of the interaction of the expanding envelope of the flaring WD with the companion in [348].

Results of the most recent calculations of the accretion of $\mathrm{H}$ up to the accumulation of a Helayer prone to explosion through thousands of small-scale outbursts, which do not erode the dwarf, are controversial. Idan et al. [307] found that accretion of hydrogen onto massive ( $\geq 1) M_{\odot}$ WD at $\dot{M} \sim 10^{-6} M_{\odot} \mathrm{yr}^{-1}$ leads to a strong flash removing all accumulated helium, instead of expected steady growth of accreted layer. In fact, since optically-thick wind is considered in the model, effective rate of He-accumulation via H-burning is only about $3 \times 10^{-7} M_{\odot} \mathrm{yr}^{-1}$ and WDs in the model of Idan et al. are in the strong He-flashes regime. On the other hand, Newsham et al. [525] claim that WD with $0.70,1.00$, and $1.35 M_{\odot}$ accreting at similar $\dot{M}$ can continuously grow in mass despite recurrent helium flashes and, hence, can be progenitors of SNe Ia. But we note that in the computations of Newsham et al. the accreted matter does not interact with the core material; this seems to be at odds with the general wisdom suggesting that diffusion or other mixing processes (e.g., shear mixing) should occur at the interface.

As already mentioned, Bours et al. [64] attempted to compare the rates of SNe Ia produced via the SD channel using several sets of retention efficiencies and values of the common envelope parameter $\alpha_{\mathrm{CE}} \lambda$ or $\gamma$-parameter of Eq. (59) employed by different groups in the population synthesis codes. For a Milky-Way like galaxy, none of the computations produced an SNe Ia rate larger than $\sim 10 \%$ of that inferred for the galaxy $-(4 \pm 2) \times 10^{-3} \mathrm{yr}^{-1}$ [87]. Claeys et al. [112] have shown that SD SNe Ia start to dominate SN Ia rate if common envelopes are inefficient $\left(\alpha_{\mathrm{CE}}=0.2\right)$; the rate of SNe Ia also then becomes comparable to the rate in the field-galaxy dominated sample, but remains a factor of three lower than the rate in galaxy clusters. Only unrealistic unlimited accretion onto WDs allows one to obtain an SNe Ia rate compatible with the observed galaxy-cluster rate.

Yoon and Langer [861, 862] and Hachisu et al. [260] have shown that if accreting WDs rotate differentially, their mass can grow without explosion up to $M \approx 2.3 M_{\odot}$, unless they lose angular momentum through secular instabilities. Supernova explosions happen during the spindown of such a WD ("spin-up/spin-down" scenario). If true, this is a possible way to accumulate enough mass for SN Ia in the SD-scenario and even to provide an explanation for some "superChandrasekhar" SN Ia. Conclusions of Yoon and Langer were questioned by Piro [591] who claims that baroclinic instabilities and/or the shear growth of small magnetic fields provide sufficient torque to bring the WD very close to rigid-body rotation in a time shorter than the typical accretion time scale; in the latter case the mass of the non-exploding WD is limited by $\approx 1.5 M_{\odot}$ [169], leaving no room for efficient mass accumulation. Thus, the "spin-up/spin-down" scenario is still highly questionable.

There is ample observational evidence that at least a fraction of SN Ia is not produced by semi-detached systems or systems with a detached giant (symbiotic stars). The major objection to the SD scenario comes from the fact that no hydrogen is observed in the SNe Ia spectra, while it is expected that up to several tenths of $M_{\odot}$ of H-rich matter may be stripped from the companion by the SN shell, see [563, 436, 692] and references therein. Hydrogen, if present, may be discovered both in very early and late optical spectra of SNe and in radio- and X-ray [60, 413]. Radio emission is produced by a synchrotron mechanism from the region of interaction between the high-velocity SN ejecta and the much more slowly moving circumstellar medium formed by the wind of the SN precursor or the wind of its companion [103, 102]. The peak monochromatic emission depends on the ratio of the rate of mass loss in the wind and its velocity $\dot{M}_{w} / u_{w}$ and allows one to estimate the pre-explosion $\dot{M}_{w}$. Panagia et al. [565] find a $2 \sigma$ upper limit of $\sim 3 \times 10^{-8} M_{\odot} \mathrm{yr}^{-1}$ on a steady 
pre-supernova mass-loss rate for $27 \mathrm{SNe}$. This excludes relatively massive companions to WDs. On the other hand, such $\dot{M}$ do not exclude systems, in which a fraction of mass lost by low-mass companions is accreted onto WDs and retained (see above).

Non-accreted material blown away from the system before the explosion would become circumstellar matter (CSM), hence the detection of CSM in SN Ia spectra would lend credence to the SD-model. Ionization and latter recombination of CSM would manifest itself as time-variable absorption features detectable (in principle) via multi-epoch high-spectral-resolution observations. However, it was noted that for confidential differentiation between CSM and ISM features for an individual SN, multi-epoch observations are needed; this condition was not always satisfied in early CSM detection reports. Currently, it is estimated that $18 \pm 11 \%$ of events exhibit time-variability that can be associated with CSM [742]. However, immediately after the very first report of CSMdiscovery in SN 2006X [572], Chugai [110] noted that for the wind densities typical for red giants, the expected optical depth of the wind in $\mathrm{Na}$ I lines is too small for their detection, while under the same conditions the optical depth of the Ca II $3934 \AA$ absorption line is sufficient for detection, and concluded that the Na I and Ca II absorption lines detected in SN 2006X could not be formed in the red giant wind and are most likely related to clouds at distances exceeding the dust evaporation radius $\left(>10^{17} \mathrm{~cm}\right)$. The problem of the interpretation of sodium-line-variability observations still remains open.

Recently, Kasen [340] suggested an important test of possible collisions of SN ejecta with companion stars, by observing emissions from the impact of shocks and SN debris due to dissipation of the kinetic energy and reheating of the gas, occurring in the immediate vicinity of the star, at $\sim\left(10^{11}-10^{13}\right) \mathrm{cm}$. Immediately after the explosion, an X-ray outburst with $L \sim 10^{44} \mathrm{ergs} / \mathrm{s}$ at $(0.1-2) \mathrm{keV}$ lasting from minutes to hours is produced. Later, radiative diffusion from deeper layers of the shock-heated ejecta produces optical/UV emissions that exceed the radioactively-powered luminosity of the SN for a few days after the explosion. The properties of the emission provide a straightforward measure of the distance between the stars and the companion's radius (in the case of RLOF). The light-curves modeled by Kasen for different companions are shown in Figure 16. The effect is prominent only for viewing angles looking down upon the shocked region $(\theta \sim 0)$ and, thus, may be found in statistical studies. Bianco et al. [46] applied Kasen's models to a sample of 87 spectroscopically-confirmed SNe Ia from the Supernova Legacy Survey [16] and ruled out the contribution from white dwarf - red giant binary systems (separation $a \sim 10^{13} \mathrm{~cm}$ ) to SNe Ia explosions greater than $10 \%$ at the $2 \sigma$ level, and greater than $20 \%$ at the $3 \sigma$ level. As noted by Kasen, optical detection of $\sim 1 M_{\odot}$ main-sequence companions $\left(a \sim 10^{11} \mathrm{~cm}\right)$ will be challenging, requiring the measurement of subtle differences in the light curves at times $\leq 2$ days. Hayden et al. [272] applied the SN Ia light-curve template to 108 objects with well-confirmed early-light curves from the Sloan Digital Sky Survey (SDSS-II [209]) and found no shock signatures. This study limited the mass of putative companions to exploding objects by about $6 M_{\odot}$ and "strongly disfavors red-giant companions".

In the SD scenario, the former companion of the exploding WD is expected to survive. It must have a high spatial velocity - (100-300) $\mathrm{km} \mathrm{s}^{-1}$ [435] and a high luminosity, see Table 7.

In Table 7 we present a summary of the expected properties of companions compiled by Schaefer and Pagnotta [679] (see this paper for a brief review of attempts to find ex-companions). As for now, all attempts to find ex-companions failed (in the particular case of SNR 0509-67.5 in SMC studied by Schaefer and Pagnotta, the non-detection was at the 5- $\sigma$ level).

A very "popular" object for the search of the former companion is Tycho's SN, but even for this close remnant of a relatively recent event (AD 1572) all claimed detections were disproved, see, e.g., [355]. For another galactic SN Ia candidate - Kepler SN (AD 1604), the possible candidates with $L>L_{\odot}$ are excluded [354].

Badenes et al. [18] noted that most models for SN Ia in the SD-scenario predict optically thick outflows from the white dwarf surface with velocities above $200 \mathrm{~km} \mathrm{~s}^{-1}$. Such outflows should 

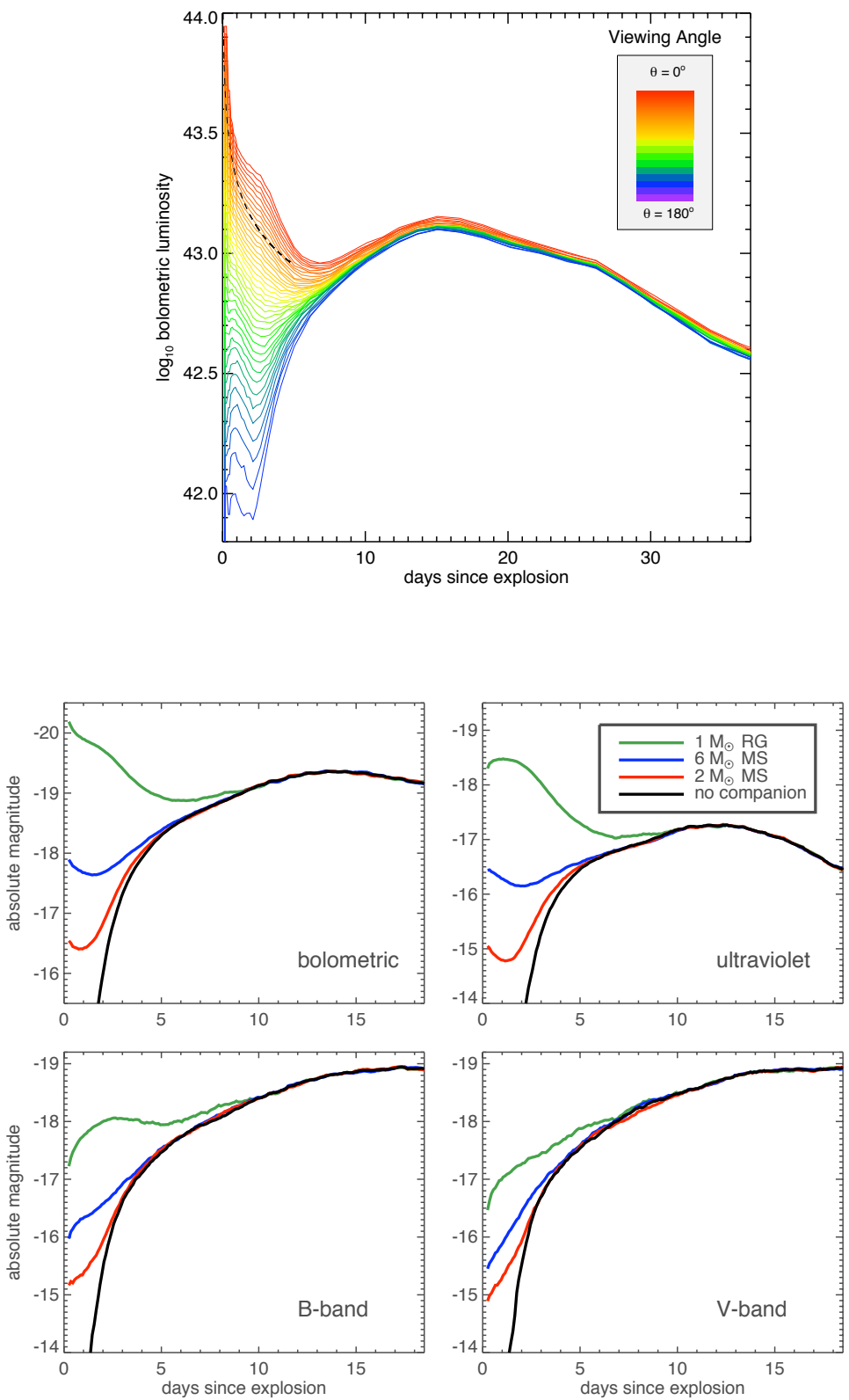

Figure 16: Upper panel: model light curve of a SN Ia having collided with a red giant companion separated by $2 \times 10^{13} \mathrm{~cm}$. The luminosity due to the collision is prominent at times $t<8$ days. The black dashed line shows the analytic prediction for the early phase luminosity. Lower panel: Signatures of interaction in the early broadband light curves of SN Ia for a red-giant companion at $2 \times 10^{13} \mathrm{~cm}$ (green lines), a $6 M_{\odot}$ main-sequence companion at $2 \times 10^{12} \mathrm{~cm}$ (blue lines), and a $2 M_{\odot}$ main-sequence companion at $5 \times 10^{11} \mathrm{~cm}$ (red lines). The ultraviolet light curves are constructed by integrating the flux in the region $1000-3000 \AA$ and converting to the AB magnitude system. For all light curves shown, the viewing angle is 0 . Image reproduced with permission from [340], copyright by AAS. 
Table 7: Properties of companions to exploding WD. After [679].

\begin{tabular}{lcclc}
\hline Candidate Class & $\begin{array}{c}P_{\text {orb }} \\
(\text { days })\end{array}$ & $\begin{array}{c}V_{\text {ex }- \text { comp }} \\
(\mathrm{km} / \mathrm{s})\end{array}$ & Surviving companion & $\begin{array}{c}M_{V} \\
(\mathrm{mag})\end{array}$ \\
\hline Double-degenerate & - & - & - & - \\
Recurrent nova & $0.6-520$ & $50-350$ & Red giant or subgiant & -2.5 to +3.5 \\
Symbiotic star & $245-5700$ & $50-250$ & Red giant & -2.5 to +0.5 \\
Supersoft source & $0.14-4.0$ & $170-390$ & Subgiant or $>1.16 M_{\odot}$ MS & +0.5 to +4.2 \\
Helium star donor & $0.04-160$ & $50-350$ & Red giant or subgiant core & -0.5 to +2.0 \\
\hline
\end{tabular}

excavate large low-density cavities around the progenitors. However, Badenes et al. found that such cavities are incompatible with the dynamics of the forward shock and the X-ray emission from the shocked ejecta in the known SNe Ia remnants in the Galaxy (Kepler, Tycho, SN 1006), LMC (0509-67.5, 0519-69.0, N103B), and in M31 (SN 1885). No sources corresponding to the preexplosion objects have been found in the archival Chandra data as yet [529]. On the other hand, e.g., spectropolarimetric observations of type Ia SN $2012 \mathrm{fr}$ suggest a simple axial symmetry of the explosion, which, together with the observed features of $\mathrm{Ca}$ and $\mathrm{Si}$ in the spectrum, is inconsistent with the DD merger scenario [473].

It was speculated that in the case of the above-mentioned "spin-up/spin-down" scenario for semidetached progenitors of SNe Ia, if the remnant of the donor star becomes a WD, during the spin-down phase it can become too dim for detection and lose traces of hydrogen [153, 328, 152]. However, the viscous time scale of a WD is still too uncertain for definite conclusions [477].

As noted first by Canal et al. [84], combinations of masses of accretors and donors enabling stable mass transfer and H-burning on the WD surface exist only for $\sim 10^{9}$ years after the starformation burst. This means that the current rate of SNe Ia with semidetached progenitors in early-type galaxies should be small, much lower than observed. This conclusion is apparently supported by the deficit of observed supersoft X-ray sources in the latter galaxies [244, 151]: while the number of supersoft sources necessary to be consistent with the rate of SNe Ia in MW or nearby galaxies is $\sim 1000$, less than about 100 are observed. We note with caution that, although WDs that accrete $\mathrm{H}$ at the rates allowing its stable burning are conventionally, following [800], identified with SSS, this inference still needs to be confirmed by rigorous modeling of the spectra of accreting WDs. Another test of the contribution of accreting WDs to the rate of SNe Ia in early-type galaxies was suggested by Woods and Gilfanov [847]: since accreting WDs, even if bloated, have photospheric temperatures $\sim 10^{5}-10^{6} \mathrm{~K}$, they are powerful sources of ionising UV emission. Then, if a significant population of steadily-burning hydrogen WDs exists, strong emission in He II $\lambda 4686$ recombination lines should be expected from the interstellar medium. Application of this test to the sample of about 11500 co-added spectra of passively evolving galaxies allowed one to limit the contribution of SD-scenarios to the total SNe Ia rate by $(5-10) \%$ [323].

\subsubsection{Merger of white dwarfs and the double-degenerate scenario for SN Ia}

A compelling scenario for the accumulation of $M_{\mathrm{Ch}}$ involves two CO WDs in a close binary system that can merge in the Hubble time due to the orbital angular momentum loss via GWR [784, 785, 300, 825]. Theoretical models suggested that a proper candidate WD pair with total mass $\gtrsim M_{\mathrm{Ch}}$ may be found among about 1000 field WDs with stellar magnitudes $V \lesssim 16-17$ [520]. This number of (super)Chandrasekhar merging pairs also appeared sufficient to obtain the observed rate of SNe Ia in a Milky-Way-like galaxy. It was speculated in [784], that, due to the fact that degenerate dwarfs expand with mass loss $\left(R \propto M^{-1 / 3}\right)$, if the mass ratio of components is 
$<2 / 3$, the merger of pairs of WDs occurs on the dynamical time scale (in a time comparable to a few orbital periods) and it was suggested that the lighter of the two dwarfs transforms into a "heavy disc" or "envelope" from which matter accretes onto the central object. This inference was confirmed by SPH calculations of Benz et al. [42] and many other studies. However, it was shown for 1D non-rotating models that the central C-ignition and SN Ia explosion are possible only for an accretion rate onto the central object $\dot{M}_{\mathrm{a}} \lesssim(0.1-0.2) \dot{M}_{\text {Edd }}$ [533], while it was expected that in the merger products of dwarf binaries $\dot{M}_{\mathrm{a}}$ is close to $\dot{M}_{\mathrm{Edd}} \sim 10^{-5} M_{\odot} \mathrm{yr}^{-1}$ because of high viscosity in the transition layer between the core and the disk [489]. For such a high $\dot{M}_{\mathrm{a}}$, nuclear burning will start at the core edge and propagate inward. If this is not the case, an off-center ignition may be due to the Kelvin-Helmholtz contraction of the inner region of the envelope on a thermal timescale of $10^{3}-10^{4} \mathrm{yr}$, which compresses the base of the envelope. Nuclear burning produces an ONeMg WD [699]. The latter will collapse without producing an SN Ia [311]. However, note that the analysis of the role of the angular momentum deposition into the central object and its thermal response to accretion in the "canonical" model led Piersanti, Tornambé and coauthors [589, 767] to the conclusion that, as a result of the WD spin-up, instabilities associated with rotation, deformation of the WD, and AML by a distorted configuration via GWR, accretion occurs not as a continuous process but episodically and, hence, the resulting "effective" accretion rate onto the WD decreases to a few $10^{-7} M_{\odot} \mathrm{yr}^{-1}$. At this $\dot{M}_{\mathrm{a}}$, a close-to-center ignition of carbon becomes possible. However, as noted by Isern et al. [310], the difficulty of this scenario is the avoidance of mass loss from the system. The off-center C-ignition can also be avoided if, after the disruption of the secondary, several conditions are fulfilled: the local maximum temperature at the interface between the core (the former primary) and the envelope (the former secondary) must be lower than the critical limit for carbon ignition, the time scale of the neutrino cooling at the core/envelope interface is shorter than the angular momentum loss time scale, the mass-accretion rate from the disc must be lower than $5 \times 10^{-6}-10^{-5} M_{\odot} \mathrm{yr}^{-1}[863]$.

The problems of the SD scenario mentioned above, the discovery of several pairs of WDs with total mass close to $M_{\mathrm{Ch}}$ and merger time shorter than the Hubble one (see Section 8), and the discovery of several SNe Ia of super-Chandrasekhar mass [291, 280, 858, 866, 676, 711, 752, 677] lent more credence to the DD-scenario. However, the current formulation of this scenario is quite different from early versions due to several circumstances. First, the accurate posing of initial conditions for mass transfer and angular momentum conservation allowed one to follow the merger process for $\sim 100$ orbits prior to the coalescence, to trace variation of the accretion rate during this phase, and to show that explosive events are possible before the coalescence or shortly after the merging $[496,166,255]$. Second, it was realized that the stability of mass transfer also depends on the efficiency of spin-orbit coupling [464]. In addition, if the circularization radius of the accretion stream is less than the accretor's radius, a direct impact occurs. Otherwise, the accretion proceeds via disk. Analytical estimates of different regimes of mass exchange in WD binary systems are presented in Figure 17.

Yet another very important, even crucial, fact concerns the role of the possible detonation of He. It was found by Livne and Glasner $[439,440]$ that accretion of He onto a $(0.6-0.9) M_{\odot} \mathrm{CO}$ WD at a rate close to $10^{-8} M_{\odot} \mathrm{yr}^{-1}$ results in the accumulation of a degenerate He layer, which detonates when its mass becomes $\sim 0.1 M_{\odot}$. The helium burning sweeps around the WD and converging shock waves initiate a compression wave that can result in central carbon detonation. For a certain time, this model involving sub-Chandrasekhar mass accretors (nicknamed "edge-lit detonations", ELD or "double-detonations") that may occur in a MW-like galaxy at a rate of $\sim 10^{-3} \mathrm{yr}^{-1}$, was considered as one of the alternative mechanisms for SD or DD scenarios for SNe Ia explosions. But it was shown by Höfflich et al $[286,285]$ that the behavior of light curves produced in this model did not resemble any of the known by mid-1990ies SNe Ia, and this mechanism was rejected. However, it was discovered later that there exists a rather numerous class of events, called SN Iax, observational properties of which are, to a certain extent, consistent with the model in which a 


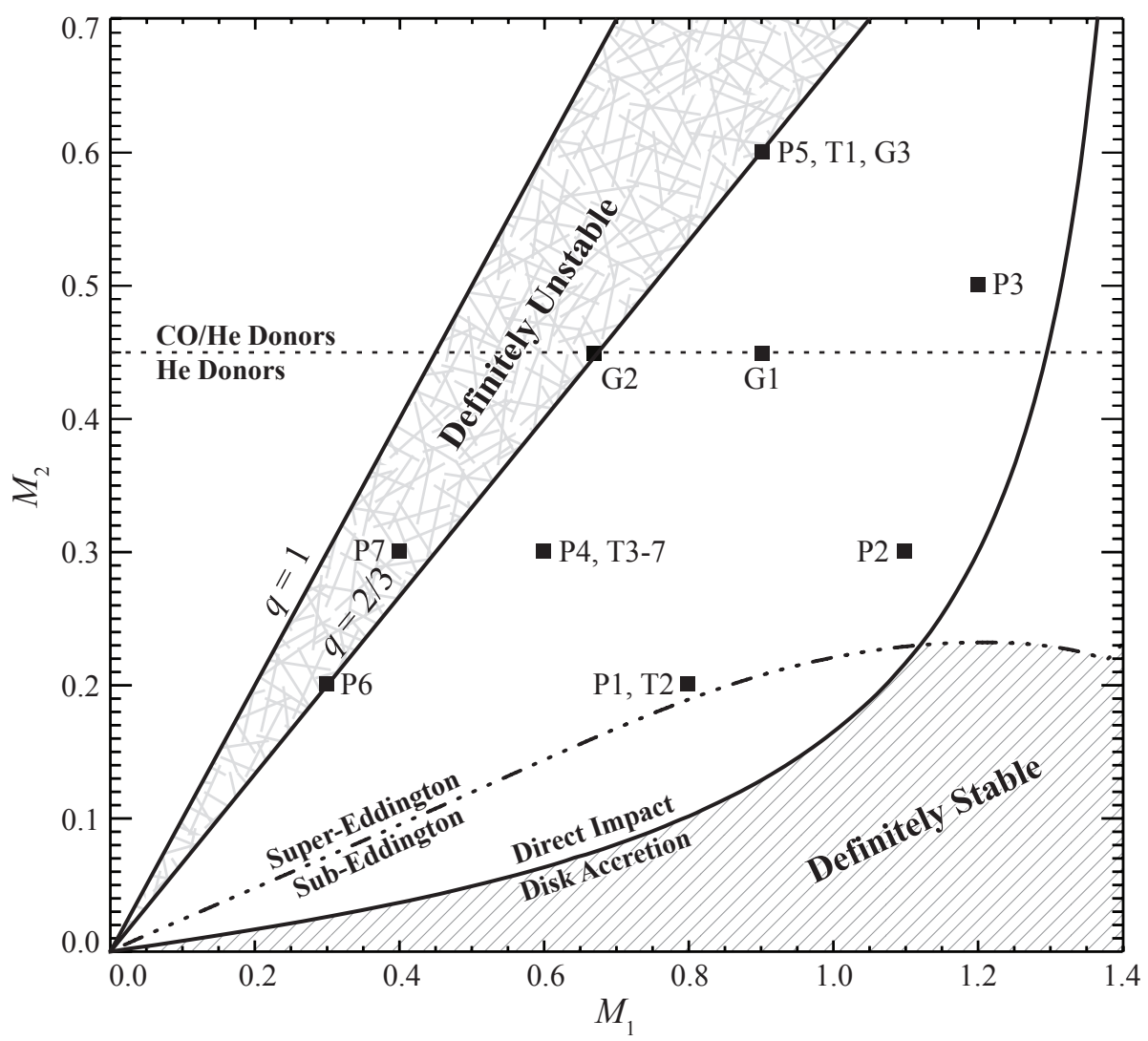

Figure 17: Estimates of regimes of mass transfer in WD binaries. Instantaneous tidal coupling is assumed. For longer time scales of tidal coupling, the stability limit in the plot shifts down [464]. The filled squares mark initial positions of the models studied in the quoted paper. Image reproduced with permission from [129], copyright by AAS.

CO WD accretes from a He-star, the burning starts in the center, the flame propagates to the surface, ejects some matter, but then damps out (called the "failed deflagration" scenario). Such SNe presumably do not consume the whole WD and leave bound remnants [203].

Interest in the "double-detonation" model re-surged in relation to the outcome of He accretion on a WD in interacting double degenerates [49]. The studies by Fink et al. [199] show that He detonation triggers core detonations in models ranging in core mass from 0.810 up to $1.385 M_{\odot}$ with corresponding shell masses from 0.126 down to $0.0035 M_{\odot}$. Similar estimates were obtained by Moll et al. [492]. It is possible that even the least massive CO WD $\left(0.45 M_{\odot}\right)$ may experience double detonations if accreted layers of He are sufficiently massive, close to $0.2 M_{\odot}$, as is shown by Sim et al. [713]. However, we note that the robustness of these results was questioned by Waldman et al. [818], since triggering a detonation must be investigated on scales much smaller than those used, e.g., by Fink et al. [199]. Nevertheless, it is quite possible that the primary objects for the DD-scenario may be pairs of CO-accretors and helium or hybrid donors.

Currently, systems with direct impact are the best studied (Figure 17). As found by Guillochon et al. [255] and later explored in more detail by Dan et al. [130, 128], in the direct impact systems He detonations in the torus of the accreted He with mass $\lesssim 0.1 M_{\odot}$ can occur several orbits before merging, if the nuclear timescale $\tau_{3 \alpha}$ becomes shorter than the local dynamical timescale $\tau_{\text {dyn }}$. If the subsequent shock compression is strong enough, the second detonation may follow in the $\mathrm{CO}$ 
core and lead to SN Ia. Another possibility is "surface detonation" in the layer of accreted matter immediately after merging with the subsequent central detonation.

In Figure 18 we show a "map" of the outcomes of mergers of white dwarfs after Dan et al. [128]. These authors systematically explored 225 WD models with different mass and chemical composition. Referring the interested reader for more details about the assumptions, computations and results to the original paper, we briefly consider the merger products shown in the Figure (moving from bottom left to upper right). Note that some of the results were well known earlier.

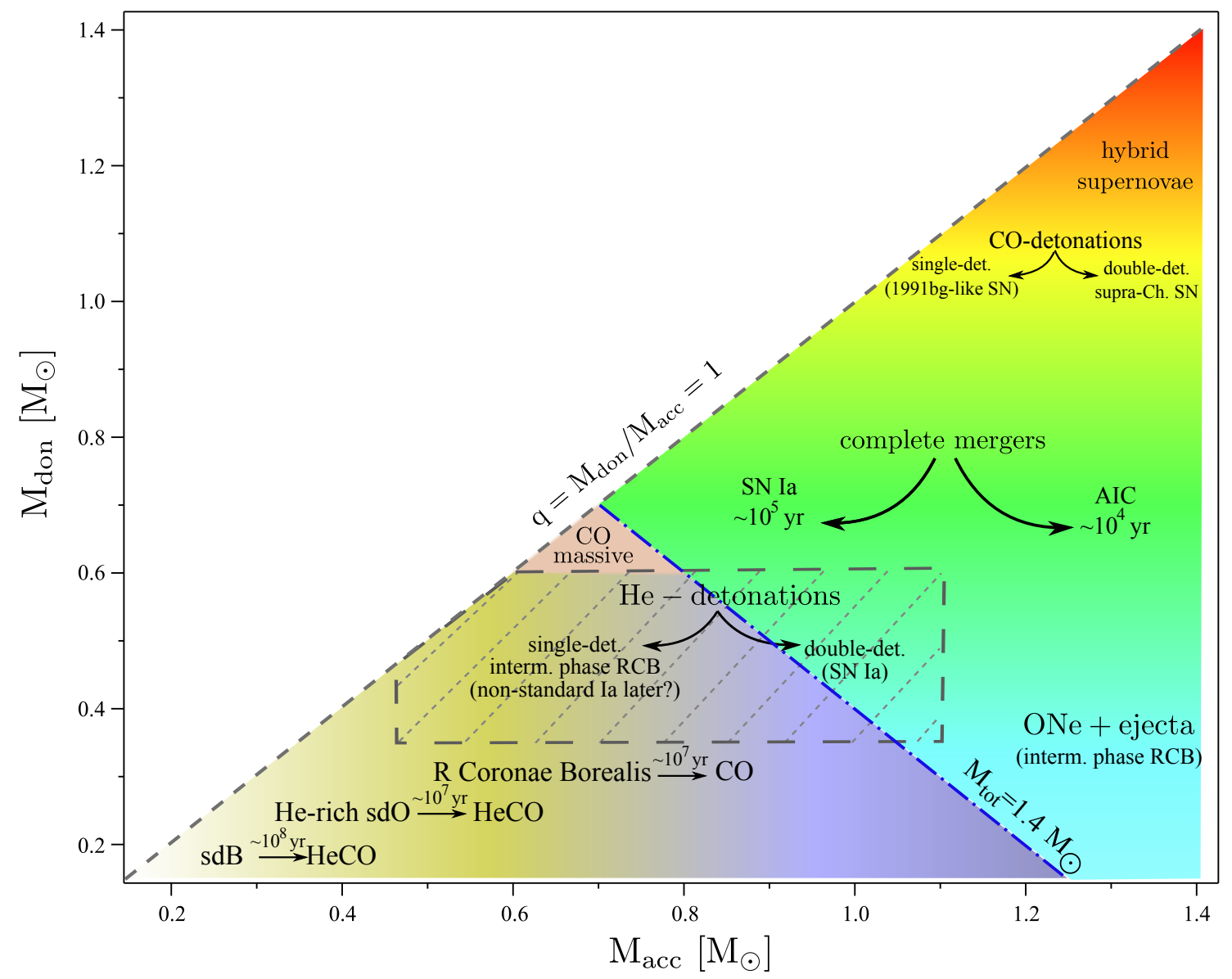

Figure 18: Outcomes of merger of WD binaries depending on the mass and chemical composition of the components. Systems in the hatched region are expected to experience He-detonations during mass transfer or at the time of merger. The numbers near the arrows indicate relevant timescales. Image reproduced with permission from Figure 1 of [128], copyright by the authors.

If the mass of the merger product of two He WDs exceeds $(0.38--0.45) M_{\odot}$, the He burns in flashes propagating inward and, finally, ignites in the core [667]. The object turns into a low-mass compact He star. If the mass is larger than $\approx 0.8 M_{\odot}$, an extended envelope forms after core He exhaustion [552]. The final product is a CO WD with a relatively thick He mantle. Low-mass He stars formed via merger are identified with single sdB stars. Mergers of two hybrid or a CO WD and a He WD with the total mass lower than $\approx 0.8 M_{\odot}$ are suggested to produce sdO subdwarfs [743]. As a confirmation of the merger scenario, one can consider the discovery of a rapidly rotating sdB star SB 290 [228]. On the other hand, recently it was shown that the merger of two He WDs with 
mass as small as $0.24 M_{\odot}$ may result in the dynamical He-burning and, possibly, in detonation [287]. Incomplete He-burning is expected in the majority of WD explosions in this case, and ${ }^{40} \mathrm{Ca}$, ${ }^{44} \mathrm{Ti}$, or ${ }^{48} \mathrm{Cr}$, rather than ${ }^{56} \mathrm{Ni}$ will be the predominant burning products.

The merger products of $\mathrm{CO}$ and He WDs with total mass exceeding $\approx 0.8 M_{\odot}$ form He giants. It is suggested that this is one of the ways to form $\mathrm{R} \mathrm{CrB}$ stars [825, 306]. Another path to $\mathrm{R}$ CrB stars may involve the merger of a CO WD and a hybrid WD $[668,566]$. The fate of $\mathrm{R}$ CrB stars is determined by the "competition" between the core growth due to He-burning in the shell and mass loss from an extended envelope. In rare cases where $\mathrm{CO}$ core accumulates $M_{\mathrm{Ch}}$, a peculiar supernova explosion can be expected. Otherwise, a massive CO WD forms.

There is a small patch in Figure 18 occupied by CO WD binaries with total mass lower than $M_{\mathrm{Ch}}$. It is expected that the merger of their components will result in the formation of a massive single WD (see [322] and references therein). Several unusually massive WDs - GD 362 [223], RE J0317-853 [393], which have mass estimates close to the Chandrasekhar mass, are potential descendants of these pairs.

Helium detonations can result from the merging of WD pairs with parameters lying within the hatched region in Figure 18. These include CO accretors and most massive helium and hybrid WD. Double-detonations are possible for most massive objects. The known problem of this mechanism of production of sub-Chandrasekhar SN Ia is that to make the modeled values consistent with observations of SN Ia, a fine tuning of He and $\mathrm{C}$ abundances in the accreting matter is required [387]. It is important to note that the newly-formed He WDs have thin hydrogen envelopes that may be stably transferred onto the companion prior to the merger. As found by Shen et al. [700], this material is ejected from the binary system and sweeps up the surrounding interstellar medium $10^{3}-10^{4}$ yr before the SN Ia explosion. The resulting circumstellar medium profiles closely match the ones found in some SN Ia.

As mentioned above, the merger of CO WD pairs with $M_{\text {tot }} \geq M_{\mathrm{Ch}}$ may result in SN Ia if certain conditions on the accretion rate at the core/envelope interface are fulfilled [589, 767, 863]. According to Dan et al. [128], the merging CO WDs detonate only if $M_{\text {tot }} \gtrsim 2.1 M_{\odot}$. Then they may be identified with super-Chandrasekhar SN Ia. However, we note that the consideration of abundances of elements synthesized in SNe Ia places certain constraints on the exploding object mass. For instance, the solar abundance of $\mathrm{Mn}$ is consistent with the assumption that about $50 \%$ of all SNe Ia are explosions of near-Chandrasekhar mass WDs [686].

Finally, it is possible that the merger involves a CO and an ONe WDs. Such (very rare) mergers can give rise to "hybrid SNe" which, in the presence of a He layer, will manifest themselves as a kind of SN Ib or, if the He-layer is absent, as Ia.

We should mention that there exist merger calculations leading to SN Ia for configurations that did not produce explosions in the simulations by Dan et al. [128].

Pakmor et al. [560] simulated a merger of $1.1 M_{\odot}$ and $0.9 M_{\odot} \mathrm{CO}$ WD with $0.01 M_{\odot}$ surface layers of $\mathrm{He}$ and found that the merger is violent, and He detonation forms close to the surface of the core of the primary in the region compressed by a shock in the He layer after only 7 orbits, i.e., much earlier than in the study of Dan and coauthors, and also triggers detonation of $\mathrm{C}$, not encountered for this combination of dwarfs in the study of Dan et al. Based on their result, Pakmor et al. claim that the merger of CO WDs with low-mass He envelopes or a CO and a He WD thus provide a unified model for normal and fast-declining SN Ia. Kromer et al. [388] were able to reproduce the early-time observables of a subluminous SN Ia with slowly-evolving light curve by violent merger of "naked" $\mathrm{CO} \mathrm{WD}$ of $0.9 M_{\odot}$ and $0.76 M_{\odot}$, which leads initially to the local Cignition at the hottest (compressed and heated) region at the accretor surface; then detonation develops around the hot bubble and consumes the entire merged structure.

We note that Scalzo et al. [678] estimated that $(0.9-1.4) M_{\odot}$ were ejected by 13 SN Ia studied by them. The correlation of ejected mass and ${ }^{56} \mathrm{Ni}$ in this set of SN Ia in most of the cases did not completely comply with the double-detonation model (e.g., [199]). The conclusion of Scalco 
et al. is that at least two formation channels, one for sub-Chandrasekhar mass SN Ia and another for Chandrasekhar mass and more massive SN Ia, are required. An interesting new channel to SN Ia discussed in this respect are direct collisions of WD, which are possible in triple systems consisting of two white dwarfs accompanied by a third star, thanks to Kozai resonances, which can reduce the time to the merger or collision [350, 399, 630]. Both sub-Chandrasekhar-mass and super-Chandrasekhar-mass SNe Ia could arise through this channel. This inference finds support in the discovery of doubly-peaked ("two-horn") line profiles in several SN Ia [159]; such profiles are expected if two WD detonate [399].

Having in mind still uncertain conditions for the initiation of C-burning, Moll et al. [491] and Raskin et al. [628] considered SN Ia explosions initiated in the merger process of CO WD, either before disruption of the secondary (dubbed by them "peri-merger" detonations) or after complete disruption of the latter when the exploding object is surrounded by an envelope (a "disk"). An important conclusion of [491] is that in the case of "peri-merger" detonations, a model for configuration with masses $0.96 M_{\odot}+0.81 M_{\odot}$ generally reproduced normal SN Ia. Explosions of more massive configurations $\left(1.20 M_{\odot}+1.06 M_{\odot}\right.$ and $\left.1.06 M_{\odot}+1.06 M_{\odot}\right)$ yielded over-luminous SN Ia. Post-merger explosions produced peculiar SN Ia with features defined, to a great extent, by the disk structure; these SN Ia do not conform with Phillips [584] relation between the peak B-band magnitude and the decline rate of the light curve found for the "standard" SN Ia. In both cases, the observed brightness of events strongly depends on the viewing angle. The mergers of massive systems may be invoked to explain super-Chandrasekhar SNe Ia.

The difference in the results of simulations by different authors may be due to different initial conditions of models $[130,629]$ and resolution of the codes used [130, 560]. Especially important is whether the binary components corotate at merger onset. These problems clearly warrant further examination.

Note, in addition, that the intermediate mass components of close binaries, before becoming white dwarfs, pass through the stage of a helium star. If the mass of the helium star exceeds $\simeq$ $0.8 M_{\odot}$, it expands to giant dimensions after exhaustion of He in the core and can overfill the Roche lobe and, under proper conditions, can stably transfer mass to the secondary companion [301]. For the range of mass-accretion rates expected for these stars, both the conditions for stable and unstable helium burning may be fulfilled. In the former case, the accumulation of $M_{\mathrm{Ch}}$ and a SN Ia become possible, as it was shown explicitly by Yoon and Langer [860]. However, the probability of such a SN Ia is only $\sim 10^{-5} \mathrm{yr}^{-1}[67]$.

Core-degenerate scenario. As a subclass of merger scenarios for SN Ia, one may consider the "core-degenerate" (CD) scenario. In the original form this scenario involves the merger of a WD with the core of an AGB star [730] accompanied by an explosion in the presence of remainders of the AGB star, or a common envelope, or nearby shells of matter presumably ejected shortly before the explosion. It was aimed at explaining SN 2002ic with signatures of circumstellar matter in the spectrum $[437,111]$. Only 16 objects of SN 2002ic class were known at the time of writing of this review [712]. Further development of the model resulted in a picture in which the merger product is a rapidly-rotating WD with mass close to or above $M_{\mathrm{Ch}}$. The fast rotation prevents immediate explosion, which happens only after a long spin-down stage, like in the spin-up/spindown scenario [308]. The core-degenerate scenario is claimed to explain successfully, the basic features of SN 2011fe [723].

To summarize, the problem of progenitors of SNe Ia is still unsettled. Large uncertainties in the model parameters involved in the computation of the evolution leading to the SN Ia explosion and in calculations of the explosions themselves, do not allow us to exclude any type of progenitors. The existence of at least two families of progenitors is not excluded by observations (see, e.g., [458] for the latest discussion). A high proportion of "peculiar" SN Ia (36 \pm 9$) \%$ [418] suggests a large 
spread in ignition conditions in the exploding objects that also may be attributed to the diversity of progenitors. Note that a high fraction of "peculiar" SNe Ia observed and their environmental dependence [641] cast certain doubts on their accuracy as standard candles for cosmology.

\subsection{Ultra-compact X-ray binaries}

The suggested channels for the formation of UCXBs in the field are, in fact, "hybrids" of scenarios presented in Figures 7 and 9. In UCXB progenitors, the primary forms a neutron star directly via core collapse or via accretion-induced collapse (AIC) of a white dwarf, while the secondary is not massive enough to form a NS. Then, several scenarios similar to the scenarios for the systems with the first-formed white dwarf and driven by systemic AML via GWR are open. Usually, two main scenarios are considered in which either a white dwarf or a low-mass helium-star companion to the NS overflows the Roche lobe. A low-mass companion to a neutron star may also overfill the Roche lobe at the end of the main sequence and become a He-rich donor. The dominant scenario differs from one population synthesis study to another, depending, mainly, on assumptions on the initial binary parameters, common envelopes, the kick velocity of nascent neutron stars, conditions for the onset of mass transfer, retention efficiency of the matter by the NS, etc. In fact, in all cases evolution of the donors leads to their transformation into similar low-mass degenerate objects.

For the most recent studies of the origin, evolution and stability of UCXB and a review of earlier work we refer the reader to $[805,806,804,92,275]$. We specially note that in [36] it was found that the dominant accretors in UCXB can be black holes formed via AIC of a neutron star. However, this result can be a consequence of the choice of the upper limit for a neutron-star mass of $2 M_{\odot}$, close to the lower bound of current theoretical estimates for the maximum mass of NS, see, e.g., [95].

For UCXBs, like for AM CVn stars, an analysis of details of the chemical composition of donors, which show up in their optical or X-ray spectra, seems to be a promising way for distinguishing between the possible progenitors [515, 521, 374]. For UCXBs, a different chemical composition of donors may manifest itself in properties of thermonuclear explosions (type I X-ray bursts) that occur on the surface of the accreting neutron stars with low magnetic fields [47]. We discuss this point in more detail in Section 9 both for UCXB and AM CVn stars.

In globular clusters, UCXBs are most likely formed by dynamical interactions, as first suggested by Fabian et al. [182]; see, e.g., [317, 441] and references therein for the latest studies on the topic. 


\section{Observations of Double-Degenerate Systems}

Interrelations between observations and theoretical interpretations are different for different groups of compact binaries. Cataclysmic variables like novae have been observed for centuries, their loweramplitude cousins (including AM CVn-type stars) for decades, see [821] for a comprehensive historical review. Their origin and evolution found theoretical explanation after effects of common envelopes and orbital angular momentum loss, in particular via gravitational waves radiation and magnetic braking, were recognized in the late 1960-1980s [551, 553, 784, 837, 810]. As of February 1, 2006 when the atlas and catalogue of CVs by Downes et al. [161, 162] were frozen, they contained about 1600 objects, but the number of known and candidate CVs is continually growing, see, e.g., [163]. Orbital periods were measured for about $1100 \mathrm{CVs}$; see the online catalogue by Kolb and Ritter [646, 645]. In particular, very recent discoveries [90, 89, 395, 415] brought the number of confirmed and candidate AM CVn stars and related helium-rich CVs to over 40.

Ultracompact X-ray binaries were discovered with the advent of the X-ray astronomy era in the late 1960s and can be found already in the first published catalogs of X-ray sources (see, for instance, [239]). Their detailed optical study became possible only with 8 m-class telescopes. Currently, the known population of UCXB consists of 13 systems with reliably-measured orbital periods, three candidates with uncertain orbital periods, five without period measurement but very low optical-to-X-ray ratios, and eight faint but persistent X-ray sources [309]. The place of UCXBs in the scenarios of the evolution of close binaries, their origin and evolution were studied already before optical identification [785, 626, 778]. Here we briefly consider information on the known population of detached close white dwarf and subdwarf binaries with WD companions. Interacting double-degenerate stars will be discussed in Sections 9 and 11.

\subsection{Detached white dwarf and subdwarf binaries}

Unlike CVs and UCXBs, the existence of close detached white dwarfs (double degenerates, DDs) was first deduced from the analysis of scenarios for the evolution of close binaries [824, 784, 785, 300, 825]. It was also suggested that DDs may be precursors of SNe Ia. This theoretical prediction stimulated optical surveys for close DDs, and the first such binary was detected in 1988 by Saffer, Liebert, and Olszewski [665]. However, a series of surveys for DDs performed over a decade [648, $66,206,462,666]$ resulted in only about a dozen of definite detections [460].

The major effort to discover close DDs was undertaken by the "ESO Supernovae Ia Progenitors surveY (SPY)" project (PI - R. Napiwotzky): a systematic radial velocity survey for DDs with the UVES spectrograph at the ESO VLT (see [501, 373, 502] for the design of the project and [503] for the latest summary of its results). The project was aimed at discovering DDs as potential progenitors of SN Ia, but brought, as a by-product, an immense wealth of data on physical parameters, kinematics etc. of white dwarfs and subdwarfs, see e.g., [373, 372, 186, 230, 573, 814, 339, 229]. Theoretical models predicted that it can be necessary to search for binarity of up to 1000 field WDs with stellar magnitude $V \lesssim 16-17$ to find a WD with a mass close to $M_{\mathrm{Ch}}$ [520]. More than 1000 white dwarfs and pre-white dwarfs were observed (practically all white dwarfs brighter than $V \approx 16.5$, available for observations from the ESO site in Chile). SPY increased the number of detected DDs tremendously to more than 150. Their system parameters are continuously determined from follow-up observations. As well, among the objects detected by SPY there appeared a large number of subdwarf stars with massive WD companions, which are of substantial relevance to the SN Ia problem (see papers by S. Geier and his coauthors).

Figure 19 shows orbital periods and total masses of the currently known (February 2014) close DDs and compares them with the Chandrasekhar mass and the critical periods necessary for the binary components to merge in $10 \mathrm{Gyr}$ for a given $M_{\text {tot }}$ assuming equal masses of components. In fact, only one DD with $M_{\text {tot }}$ close to $M_{\mathrm{Ch}}$ was detected by SPY (see the figure). On the other 


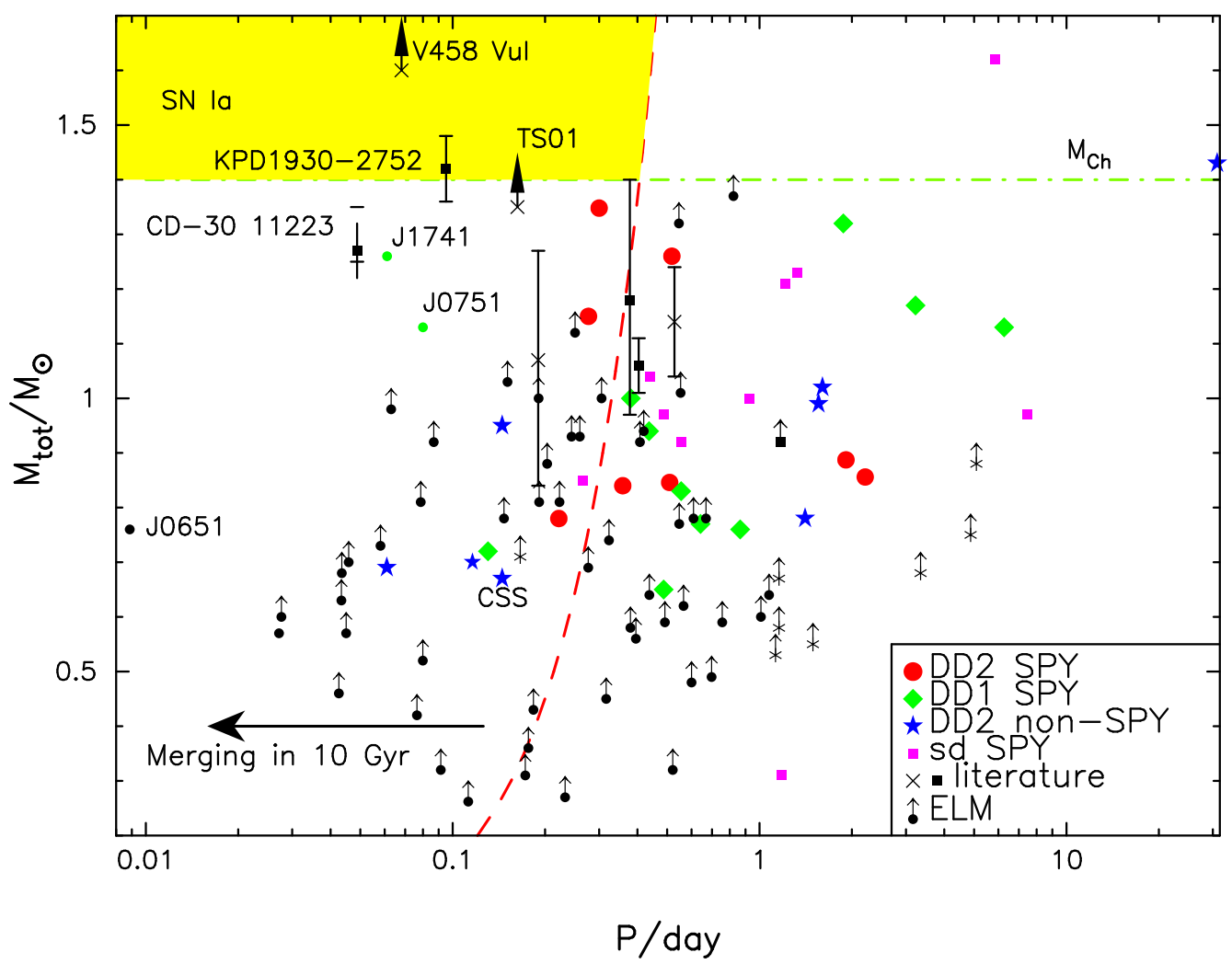

Figure 19: Known close binaries with two WD components, or a WD and a sd component. Red circles mark double-line WDs found by SPY. Green diamonds are single-line WDs found by SPY. Blue asterisks mark double-line WD discovered in surveys other than SPY. Magenta squares are sd + WD systems from SPY. Black crosses and small squares are single-line WD and sd found by different authors. Filled black circles are extremely low-mass WD (ELM) for which, typically, only one spectrum is observed. For singleline systems from SPY we assume inclination of the orbit $i=60^{\circ}$, for other single-line systems we present lower limits of the total mass and indicate this by arrows. Green circles are double-lined ELM WD suggested to be definite precursors of AM CVn stars Several remarkable systems are labeled (see text for details). The "merger" line is plotted assuming equal masses of the components. This is an update of the plot provided by R. Napiwotzki for the previous version of this review.

hand, more than a hundred new close WD binaries were discovered by SPY (most of them are still awaiting follow-up observations for the determination of parameters).

In the meantime, several other interesting objects were discovered, including, PN G135.9+55.9= TS01, first detected as the nucleus of a planetary nebula (PN) [769]. Later, its binarity was discovered. A study of its parameters led to a total mass estimate possibly exceeding $M_{\mathrm{Ch}}[740,768]$. Another remarkable system is V458 Vul (Nova Vul 2007 No. 1) - the nucleus of a planetary nebula with a massive WD companion, which exploded as a nova in 2007. Masses of components are estimated to be $0.58 M_{\odot}$ and $\gtrsim M_{\odot}[650,120]$. A high mass of $\mathrm{PN}$ in this system $(\sim 0.2) M_{\odot}$ points to its formation via ejection of a common envelope about $14000 \mathrm{yr}$ ago [833]. Components of the system will merge due to the orbital angular momentum loss via GWR in $\sim 10^{7} \mathrm{yr}$. By this time, the current nucleus of PN will turn into a WD. Mass exchange after contact is expected to be unstable (see Figure 17); this system is currently considered to be the most likely precursor of SN Ia. 
Another possible SN Ia precursor is KPD $1930+2752$ - an sdB star with an unseen massive WD companion. The estimated total mass of the system ranges from $1.36 M_{\odot}$ to $1.48 M_{\odot}$ [231]. In $\simeq 200 \mathrm{Myr}$ prior to the merger, the present sdB star can turn into a WD, and then a mass-transfer phase will ensue. Its stability depends on the efficiency of the tidal coupling (Figure 17). If the sdB-star still remains in the core He-burning stage at RLOF, an AM CVn system will be formed.

The binary system CD-30 11223 (GALEX J1411-3053) is the shortest $P_{\text {orb }}$ sdB + WD pair known to date [808]. Estimated masses of components are $M_{\mathrm{sdB}}=0.44-0.48 M_{\odot}$ and $M_{\mathrm{WD}}=$ $0.74-0.77 M_{\odot}$. The time before RLOF by the sdB star $(\approx 30 \mathrm{Myr})$ is apparently too short for its transformation into a WD, and stable mass transfer onto the WD upon RLOF may be expected. Thus, this system is a potential precursor to an AM CVn star.

The most numerous population (58 objects) in Figure 19 is of extremely low mass white dwarfs (ELM) - helium white dwarfs with $M \lesssim 0.25 M_{\odot}$ found in a targeted spectroscopic survey (see [72] for a summary of results; data for the plot taken from this paper and [360, 241]. Most of them have unseen heavier companions, most probably another WDs, while in some cases even NSs are suspected. Some may have brown-dwarf companions. About half of ELM WD will merge with their companions in less than the Hubble time. As Figure 20 shows, some of them, upon merger (which may take longer than the Hubble time) can start a stable mass transfer, i.e., can form AM CVn stars. The most remarkable of ELM WDs is SDSS J065133.338+284423.37 (J0651) [359, 73], the shortest known detached WD, for which relativistic decay of the orbital period is detected. It is expected that it will be possible to detect some ELM stars by space-born GW detectors [358].

Yet more important recently-discovered objects are the eclipsing ELM WD SDSS J075141.18014120.9 (J0751) and ELM WD SDSS J174140.49+652638.7 (J1741) [360]. The masses of the components of $\mathrm{J} 0751$ are $0.168 M_{\odot}$ and $0.97_{-0.01}^{+0.06} M_{\odot}$, the orbital period is $P_{\text {orb }}=0.08$ day. The binary components will merge in about $270 \mathrm{Myr}$. The parameters of J1741 are $M_{1}=0.19 \pm 0.02 M_{\odot}$, $M_{2} \geq 1.11 M_{\odot}, P_{\text {orb }}=0.06$ day, the merging time is $160 \mathrm{Myr}$. With such a low mass ratio, one can almost certainly expect a stable RLOF (see Figure 22) for J1741. For J0751, a stable mass exchange is also highly probable. This makes these two systems the first-discovered almost-certain progenitors of AM CVn stars and, possibly, He-novae (see Section 9).

The observed precursors of ELM WD can be hidden within EL CVn type binaries, which were recently identified as a separate class of objects - eclipsing binaries with short orbital periods (0.72.2) day, spectral type A primaries and very hot low-mass pre-He-WD [474]. Due to the large mass ratios of components, RLOF by the A-stars in these binaries will result in common envelopes. If components will not merge in CE, theses systems will turn into ELM WD binaries.

Evolutionary sequences for ELM WD were computed by Althaus et al. [9], who also provided interpolation formulas for fitting masses and cooling ages of ELM WD as functions of $T_{\text {eff }}$ and $\log g$.

WD binary CSS 41177 is an eclipsing $P_{\text {orb }}=2.78 \mathrm{hr}$ system with helium WD components: $M_{1}=0.38 \pm 0.02 M_{\odot}, M_{2}=0.32 \pm 0.01 M_{\odot}$ [65]. The merging time of components is $1.14 \pm 0.05 \mathrm{Gyr}$ and this system is the perfect candidate to form a single sdB star [786, 667], see Section 7.3.2.

The most massive WD binary with parameters estimated from astrometric data and optical and near-IR photometry is LHS 3236 [271]. It may be a pair of DA WD or a DA WD in a pair with a DC WD. Masses of its components are either $0.93 M_{\odot}$ and $0.91 M_{\odot}$ or $0.98 M_{\odot}$ and $0.69 M_{\odot}$ (depending on types assigned to WD). However, a binary orbital period of $4.03 \mathrm{yr}$ corresponds to the tremendously-long merging time $\simeq 2 \times 10^{13}$ Myr. 


\section{Mass ratio distribution, brightest/faintest}

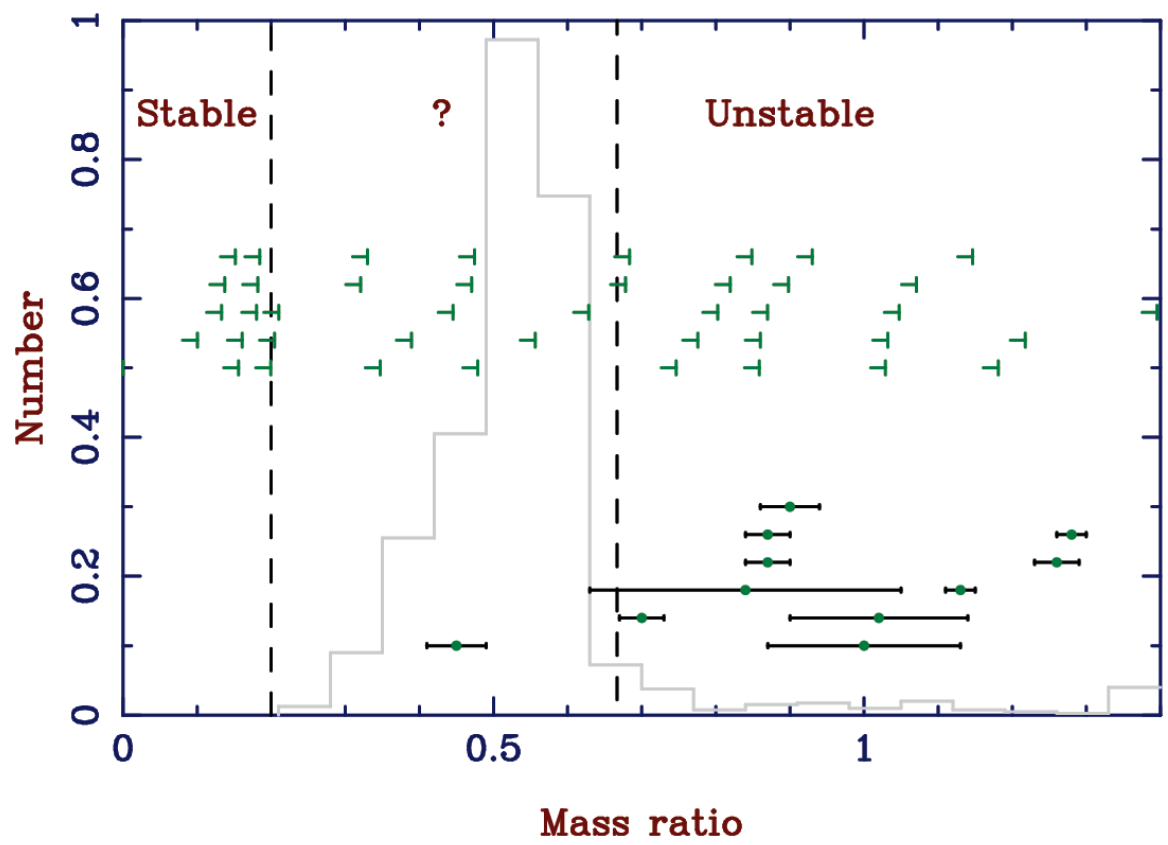

Figure 20: Mass ratios of ELM WD. Vertical lines separate binaries which will, upon RLOF, exchange mass definitely stably (possible progenitors of AM CVn stars), WD for which stability of mass-exchange will depend on the efficiency of tidal interaction, and definitely unstable stars. The latter systems may be progenitors of SNe Ia, as discussed in Section 7.3.2. Courtesy T. Marsh [459].

\section{Evolution of Interacting Double-Degenerate Systems}

As shown in the flowchart in Figure 9 and mentioned above, there are several ways to form a semidetached system in which a WD stably accretes matter from another WD or a helium star. This happens due to orbital angular momentum losses via GWR, since for the WD binary, orbital angular momentum loss is the only driver of orbital evolution, and low-mass helium stars $\left(M \lesssim 0.8 M_{\odot}\right)$ almost do not expand in the course of core He-burning. These stars, also referred to as "interacting double-degenerates" (IDD), are observationally identified with the ultra-shortperiod cataclysmic variables $\left(P_{\text {orb }}=(5-65) \mathrm{min}\right)$ of the AM CVn class. We shall use below both the terms "AM CVn stars" and "IDD". A distinctive peculiarity of AM CVn stars is the absence of signs of hydrogen in their spectra (with one possible exception), which suggests that donor stars in these binaries are devoid of hydrogen. The absence of hydrogen seems to be reliable, since the threshold for its detection is quite low: $\left(10^{-5}-10^{-2}\right)$ by the number of atoms [842, 726, 463].

Note that apart from "double-degenerate" and "helium-star" channels for the formation of AM CVn-stars, there exists the third, "CV"-channel [778, 779, 522, 596]. In the latter, the donor star fills its Roche lobe at the very end of the main-sequence stage $\left(X_{c} \sim 0.01\right)$ or just after its completion. For such donors the chemical inhomogeneity inhibits complete mixing at $M \simeq 0.3 M_{\odot}$ typical for initially non-evolved donors. The mixing is delayed to lower masses, and as a result the donors become helium dwarfs with some traces of hydrogen. After reaching the minimum orbital periods $\simeq(5-7)$ min, these binaries start to evolve to longer orbital periods. However, in this scenario the birth rate of systems that can penetrate the range of periods occupied by 
the observed AM CVn-stars, especially $P_{\text {orb }} \lesssim 25$ min, is much lower than the birth rate in the "double-degenerate" and "helium-star" channels; below we shall neglect it.

Schematically, the tracks of three families of AM CVn stars are shown in Figure 21 and compared to the track for an "ordinary" CV.

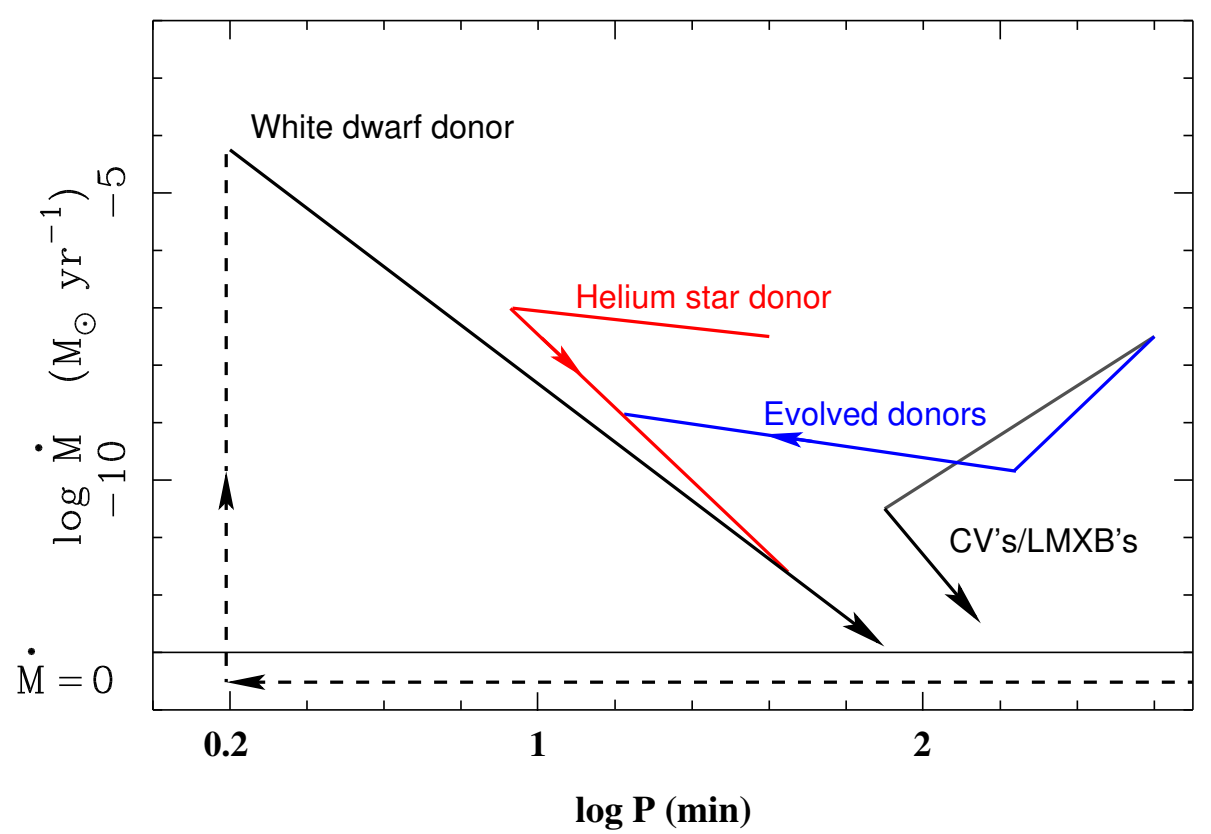

Figure 21: Sketch of the period - mass transfer rate evolution of the binaries in the three proposed formation channels of AM CVn stars (the dashed line shows the detached phase of the white dwarf channel). For comparison, the evolutionary path of an ordinary hydrogen-rich CV or low-mass X-ray binary is shown. Image reproduced with permission from Figure 1 of [521], copyright by the authors.

\section{1 "Double-degenerate family" of AM CVn stars}

The importance of AM CVn stars both for astrophysics and for physics in general stems from the fact that, because of their very short orbital periods and relative brightness due to proximity to the Sun, they are expected to be strong GWR sources and the first Galactic objects discovered by space GW interferometers like LISA (or eLISA), i.e., they will serve as "verification binaries" [744]. Besides, AM CVn stars can be precursors of SN Ia [784, 727, 662] as well as still-hypothetical order-of-magnitude-weaker explosive events called SN .Ia [48, 697, 702, 576].

A comprehensive review paper on AM CVn stars covering their observational properties and some theoretical aspects was published recently by Solheim [725]. Therefore, we shall provide below only basic information about these binaries.

In a binary with stable mass transfer, change of the radius of the donor exactly matches the change of its Roche lobe. This condition combined with the approximate size of the Roche lobe given by Eq. (35) (which is valid for low $q$ ) provides the following relation between the orbital period of the binary $P_{\text {orb }}$ and the mass $M_{2}$ and radius $R_{2}$ of the donor:

$$
P_{\text {orb }} \simeq 101 \mathrm{~s}\left(\frac{R_{2}}{0.01 R_{\odot}}\right)^{3 / 2}\left(\frac{0.1 M_{\odot}}{M_{2}}\right)^{1 / 2}
$$


and the expression for the rate of mass transfer for a semidetached binary in which the mass transfer is driven by the orbital angular momentum losses:

$$
\frac{\dot{m}}{m}=\left(\frac{\dot{J}}{J}\right)_{\mathrm{GWR}} \times\left(\frac{\zeta(m)}{2}+\frac{5}{6}-\frac{m}{M}\right)^{-1}
$$

where $\zeta(m)=d \ln r / d \ln m$. For the mass transfer to be stable, the term in brackets must be positive, i.e.

$$
\frac{m}{M}<\frac{5}{6}+\frac{\zeta(m)}{2}
$$

Violation of this criterion results in mass loss by the WD donor on a time scale comparable to $10-$ 100 orbital periods, as discussed in Section 7 and most probably, in the merging of components, if a supernova explosion is avoided. Of course, Eq. (71) oversimplifies the conditions for a stable mass exchange. A rigorous treatment must include tidal effects, angular momentum exchange, and the possible super-Eddington $\dot{M}$ immediately after RLOF by the donor and the associated common envelope formation, as well as the possible ignition of accreted helium on the WD surface [829, $269,464,246]$.

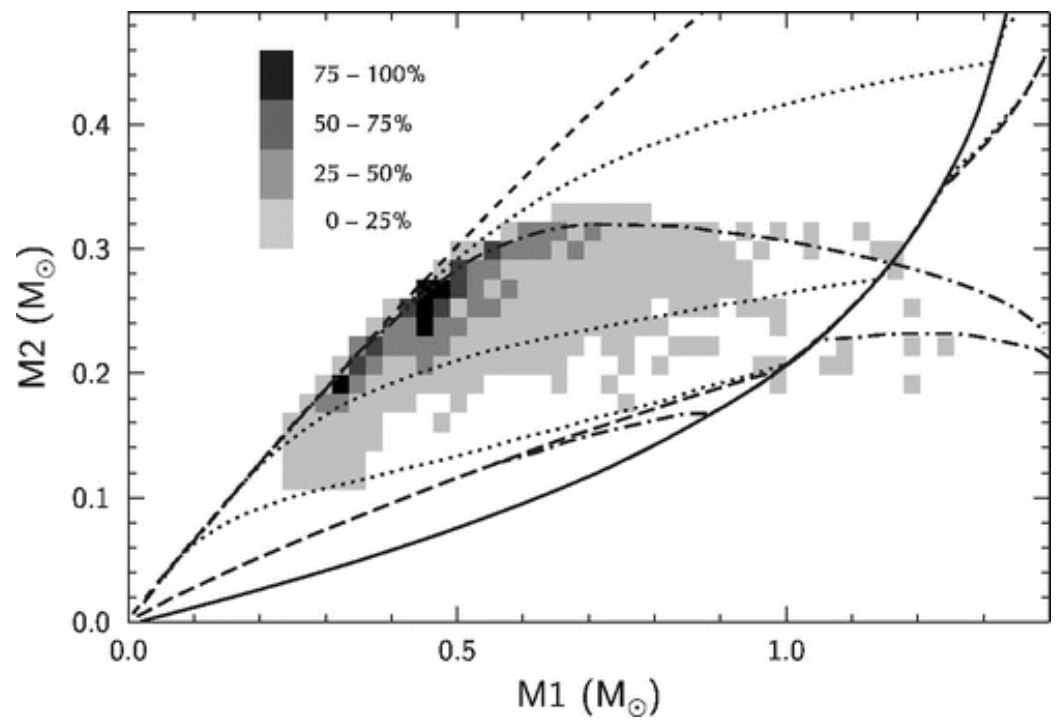

Figure 22: Birthrates and stability limits for mass transfer between close WD binaries. The shaded areas show the birth probability of progenitors of AM CVn stars in double-degenerate channel scaled to the maximum birth rate per bin of $9 \times 10^{-5} \mathrm{yr}^{-1}$ [518]. The upper dashed line corresponds to the upper limit for stable mass transfer. The lower solid line is the lower limit for direct accretion. The upper dash-dot line is the limit set by the Eddington luminosity for stable mass transfer at a synchronization time limit $\tau \rightarrow 0$. The lower dash-dot line is the limit set by the Eddington luminosity for $\tau \rightarrow \infty$, and the lower broken line is the strict stability limit for the same. The three dotted lines show how the strict stability limit is raised for shorter synchronization time-scales ranging from $1000 \mathrm{yr}$ (bottom), $10 \mathrm{yr}$ (center), and 0.1 yr (top). Image reproduced with permission from Figure 3 of [725], copyright by ASP.

Currently, only some of these effects have been studied to a certain extent. In Figure 22 the distribution of birthrates of the possible progenitors of AM CVn stars in the DD channel from [518] is over-plotted with constraints imposed by the mass exchange stability conditions, the Eddington accretion rate, and the efficiency of tidal interaction (in fact, this is the bottom part of Figure 17 applied to the population of AM CVn stars). It is clear that the substantial number of AM CVn 
stars formed via the "double-degenerate" scenario may exist only in the case of efficient tidal coupling. As shown in [518], the difference in the expected numbers of stars between two limiting cases of synchronization time $\tau \rightarrow 0$ and $\tau \rightarrow \infty$ amounts to two orders of magnitude.

AM CVn systems are so close that in some of them the accretion stream may hit the accretor directly, and the accretion disk does not form [512]. The candidate "direct impact" systems currently include the shortest orbital period known systems HM Cnc $\left(P_{\text {orb }}=5.36 \mathrm{~min}\right)$, V407 Vul $\left(P_{\text {orb }}=9.48 \mathrm{~min}\right)$, ES Cet $\left(P_{\text {orb }}=10.36 \mathrm{~min}\right)$, see [725] for discussion and references.

Until quite recently, the evolution of systems with WD donors was calculated using Eq. (70) and applying different $M-R$ relations for zero-temperature WDs close to $R \propto M^{-1 / 3}$, such as given in [882, 506, 809], ignoring detailed models of dwarfs. For He-star donors, radii were approximated by fits obtained in the evolutionary models of semidetached systems (e.g., $R \approx 0.043 \mathrm{~m}^{-0.062}$ from [776]). Early calculations of this kind may be found in [551, 812, 194, 784, 626, 748, 809, 790, $518,519,661]$. Figure 23 shows examples of the evolution of systems with a helium degenerate donor or a low-mass "semidegenerate" helium star donor and a carbon-oxygen accretor with initial masses that are currently thought to be typical for progenitors of AM CVn systems - 0.2 and $0.6 M_{\odot}$ for double degenerates and 0.4 and $0.6 M_{\odot}$ for He-star systems, respectively. In Figure 23 the mass-radius relation [809] is used for WD, while for the low-mass He-star $-M-R$ relation from [776]. The same equations are applied to obtain the model of the population of AM CVn-stars discussed in Sections 10 and 11.

Equations (25), (35), and (70) imply that for $m \ll M$ the mass loss rate scales as $M^{1 / 3}$. As a result, for all combinations of donor and accretor masses, the $P-\dot{m}$ lines form two rather narrow strips within which they converge with decreasing $m$.

The "theoretical" model of evolution from shorter periods to longer ones is supported by observations, which found that the UV luminosity of AM CVn-stars is increasing as the orbital period gets shorter, since shorter periods are associated with higher $\dot{M}$ [624]. The orbital period change $\dot{P}_{\text {orb }}=(3.07 \pm 0.56) \times 10^{-13}$ day day $^{-1}$ at the $5.4 \sigma$ level was quite recently found for the first discovered eclipsing AM CVn type binary SDSS J0926+3624 $\left(P_{\text {orb }}=28.3 \mathrm{~min}\right.$. [746]). This value of $\dot{P}$ is consistent with the mass-exchange rate close to $1.8 \times 10^{-10} M_{\odot} \mathrm{yr}^{-1}$, expected both in the "double-degenerate" and "helium-star" channels of the evolution of AM CVn stars for the period of SDSS J0926+3624. Sion et al. [717] estimated accretion rates in several AM CVn binaries by means of analyses of their far and near-UV spectra and found that in their sample; the system ES Ceti with the shortest $P_{\text {orb }}=5.4$ min has the highest $\dot{M}=2.5 \times 10^{-9} M_{\odot} \mathrm{yr}^{-1}$, while the longest orbital period system GP Com $(46.5 \mathrm{~min})$ has the lowest $\dot{M}=(3-4) \times 10^{-11} M_{\odot} \mathrm{yr}^{-1}$.

About 1/3 of AM CVn stars demonstrate outbursts [623]. The properties of an outburst are well described by the thermal-viscous disk instability model, see [379] and references therein. Crudely, disks are unstable for mass accretion rates $10^{-12} \lesssim \dot{M}_{\text {accr }} \lesssim 10^{-9} M_{\odot} \mathrm{yr}^{-1}$.

However, we should note that the time span between the formation of a pair of WDs and their contact may range from several Myr to several Gyr [790, 518]. This means that the approximation of zero-temperature white dwarfs is not always valid. $T_{c}$ of $\mathrm{WD}$ at the onset of mass transfer may be as high as almost $10^{8} \mathrm{~K}$, as estimated by Deloye et al. [142] for WD progenitors of donors of AM CVn stars from [518]. This produces differences in period distributions of stars at the onset of mass transfer: while pairs with zero-temperature WDs have narrow distribution $1.5 \leq P_{\text {cont }} \leq 6.5 \mathrm{~min}$, systems with finite entropy WDs have $1.5 \leq P_{\text {cont }} \leq 17.5 \mathrm{~min}$ and for about $80 \%$ of the model stars $P_{\text {cont }}>6.5 \mathrm{~min}$.

Deloye et al. incorporated the pure helium equation of state and the low temperature opacities in their computations of (still unique) series of full evolutionary models of mass-losing donors with different initial mass and electron degeneracy. ${ }^{30}$ Their main finding is that the evolution of the donor includes three distinct phases: (i) the onset of mass transfer in which the WD contracts,

\footnotetext{
30 The latter is characterized by the parameter $\psi=E_{\mathrm{F}, c} / k T_{c}$, where $E_{\mathrm{F}, c}$ is the electron Fermi energy, $T_{c}-$ the central temperature of a WD.
} 

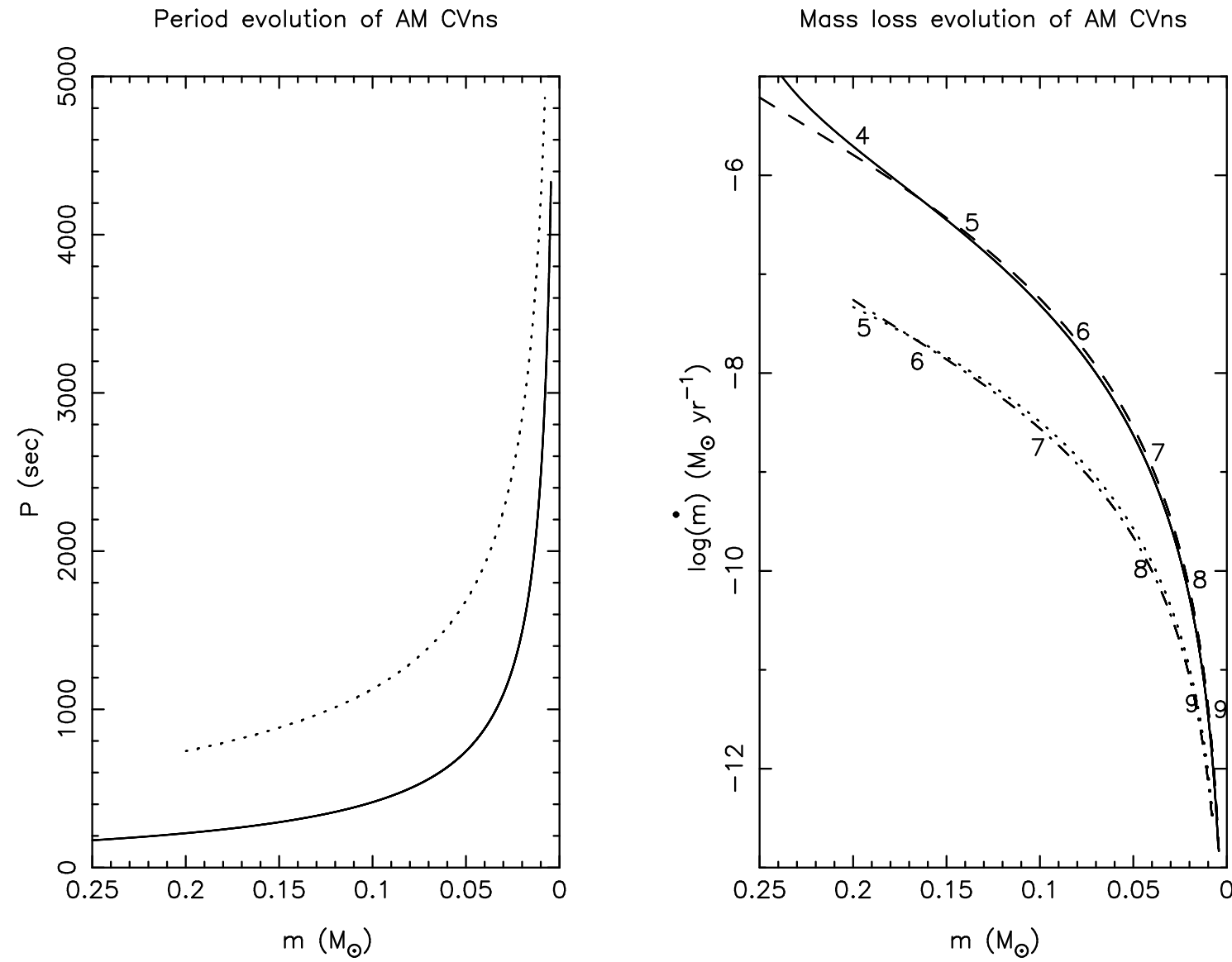

Figure 23: Examples of the evolution of AM CVn systems. Left panel: The evolution of the orbital period as a function of the mass of the donor star. Right panel: The change of the mass transfer rate during the evolution. The solid and dashed lines are for zero-temperature white dwarf donor stars with initial mass $0.25 M_{\odot}$ transferring matter to a primary with initial mass of 0.4 and $0.6 M_{\odot}$, respectively, assuming efficient coupling between the accretor spin and the orbital motion. The dash-dotted and dotted lines are for a helium star donor, starting when the helium star becomes semi-degenerate (with a mass of $\left.0.2 M_{\odot}\right)$. Primaries are again 0.4 and $0.6 M_{\odot}$. The numbers along the lines indicate the logarithm of time in years since the beginning of mass transfer. Image reproduced with permission from [512], copyright by ESO. 

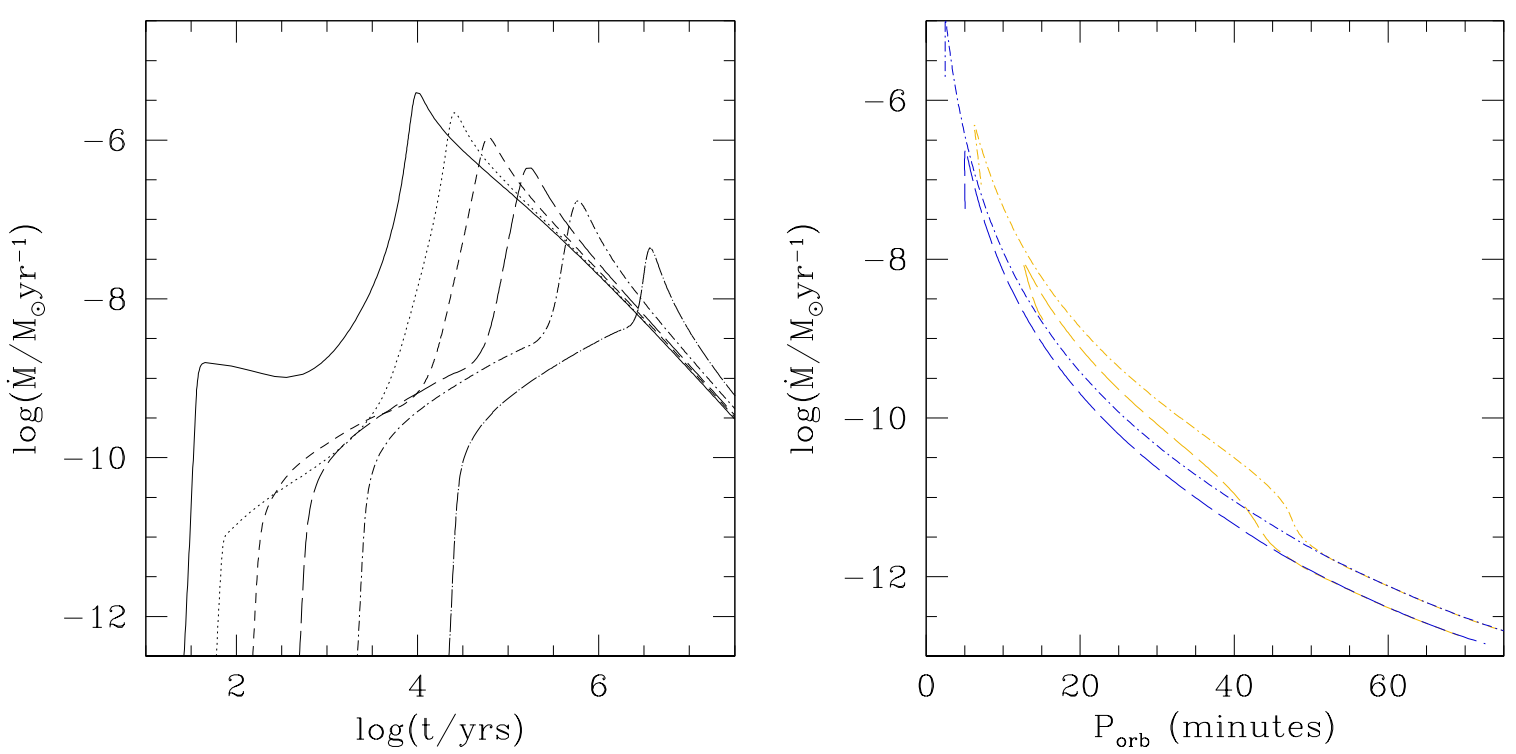

Figure 24: Left panel: the $\dot{M}(t)$ evolution of AM CVn systems with $M_{2, i}=0.2 M_{\odot}$ and $M_{1, i}=0.3 M_{\odot}$. Systems with donors having the degeneracy parameter $\log \left(\psi_{c, i}\right)=3.5,3.0,2.5,2.0,1.5$ and 1.1 are shown by the solid, dotted, short-dashed, dashed, shortdash-dotted and dash-dotted lines, respectively. Right panel: the dependence of the $\dot{M}-P_{\text {orb }}$ relation on the initial binary parameters for pairs $M_{1, i}+M_{2, i}=$ $(0.35+0.15) M_{\odot}, \log \left(\psi_{c, i}\right)=1.1$ (yellow dashed line), $M_{1, i}+M_{2, i}=(1.025+0.30) M_{\odot}, \log \left(\psi_{c, i}\right)=3.0$ (yellow dot-dashed line), $M_{1, i}+M_{2, i}=(0.35+0.15) M_{\odot}, \log \left(\psi_{c, i}\right)=1.1$ (blue dashed line), $M_{1, i}+M_{2, i}=$ $(1.025+0.30) M_{\odot}, \log \left(\psi_{c, i}\right)=3.0$ (blue dot-dashed line). Image reproduced with permission from Figures 6 and 10 of [142], copyright by the authors.

mainly due to the fact that the very outer layers are never degenerate, (ii) the phase of adiabatic expansion, and (iii) the final phase when the thermal time scale of the donor becomes shorter than the mass-loss time scale and the white dwarf cools, contracts, and evolves to a nearly degenerate configuration. Early computations were not able to account for these transitions, since mass-radius relations for zero-temperature WD were used.

In Figure 24 we show, after Deloye et al., $\dot{M}(t)$ evolution for AM CVn systems with initial masses $M_{2, i}=0.2 M_{\odot}$ and $M_{1, i}=0.3 M_{\odot}$ and the dependence of the $\dot{M}-P_{\text {orb }}$ relation on the initial binary parameters and the degeneracy parameter for pairs with different initial component masses and electron degeneracy. Note that the initial stage of decreasing $P_{\text {orb }}$ is very short and hardly observable (however, we do observe HM Cnc). At the final stage of evolution, the tracks with different initial degeneracy merge. The comparison with Figure 23 shows that the tracks for the "intermediate" adiabatic mass loss stage based on the zero-temperature $M-R$ relation and on finite entropy models do not differ significantly, justifying early calculations, while in the stage of cooling at $P_{\text {orb }}>(40-45)$ min, the mass loss rate becomes so small that stars will again be hardly observable. The main difference between the approximate and full computations arises from taking into account the initial non-zero temperature and should be reflected in $P_{\text {orb,max }}$ after contact. This may affect the expected GW signal from AM CVn stars, which must be dominated by the shortest period systems (and the closest ones), see Section 10.

Yet another point, which may slightly change the evolution of the double-degenerate family of AM CVn stars based on the "traditional" $M-R$-relation, is the possible presence of a lowmass $\left(\sim 10^{-3} M_{\odot}\right)$ but a geometrically thick (several $\left.0.01 R_{\odot}\right)$ nondegenerate layer of hydrogen supported by $p-p$ nuclear burning at the surface of the donor at RLOF. Models of mass-transfer 
in such systems $[131,337]$ show that (i) they may make contact at $P_{\text {orb }} \approx(6-10)$ min and (ii) for the first $\simeq 10^{6} \mathrm{yr}$ of evolution radii of the donors decrease and systems evolve to shorter periods, until the H-rich layer is almost shed. This may be the case of AM CVn-type star HM Cancri (= RX J0806.3+1527), which is also the star with the shortest known $P_{\text {orb }}=321.5 \mathrm{~s}[652]$ and the only member of the AM CVn class of binaries with a suspected presence of hydrogen.

\section{2 "Helium-star family" of AM CVn stars}

Precursors of He-star components of AM CVn stars with initial mass $\approx(0.35-0.80) M_{\odot}$ are $M_{\text {ZAMS }} \approx(2-5) M_{\odot}$ red giants with non-degenerate helium cores (Figure 9). Helium stars with $M \lesssim 0.80 M_{\odot}$ almost do not expand in the course of evolution (by less than 30\% [552]). Their lifetimes are comparable to the main-sequence lifetimes of their precursors (Eq. 68) and the orbital angular momentum loss via gravitational waves radiation may bring them to RLOF before the exhaustion of He in their cores [674, 302, 790]. Mass transfer proceeds steadily if the initial mass ratio of the He star and the WD is less than approximately 3 [318]. The evolution of low-mass semidetached systems with He-donors was explored in detail by Yungelson [870]. Referring the reader for details to this paper, we show in Figure 25 as an example a set of tracks for the system with initial masses of components $\left(M_{\mathrm{He}}+M_{\mathrm{WD}}\right)=(0.40+0.60) M_{\odot}$, which have at the formation (immediately after completion of common envelope evolution) orbital periods from 20 to $130 \mathrm{~min}$. The initial abundances of He and heavy elements at "He-ZAMS" are assumed to be 0.98 and 0.02 , respectively. RLOF in these systems happens at orbital periods of about 20 to 30 min when He abundance in their cores ranges from 0.98 to 0.066 , from the least evolved models at RLOF to the most evolved ones. The initial stage of increasing $\dot{M}$ lasts for $\sim 10 \mathrm{Myr}$ only. The stars reach minimum periods of $\sim 10 \mathrm{~min}$. Their mass at this time is close to $0.2 M_{\odot}$. As shown in [870], the $P_{\text {orb }}-\dot{M}$ relation very weakly depends on the total mass of the system. Mass loss leads to the cooling of stellar interiors and quenching of nuclear burning by the time when the minimum of $P_{\text {orb }}$ is reached, see Figure 26. After the minimum of $P_{\text {orb }}$, the chemical composition of the matter transferred to the companion is "frozen" and does not change during the subsequent evolution. In the course of further evolution the matter of donors becomes increasingly degenerate, and their mass-ratio relation approaches that of WD.

As mentioned in Section 7, until recently it was deemed that, at accretion rates of He onto a WD below several units of $10^{-8} M_{\odot} \mathrm{yr}^{-1},(0.1-0.3) M_{\odot}$ of He accumulates at the WD surface and then detonates; converging detonation waves propagate to the center of the accretor and ignite carbon detonation close to the center. Prior to the minimum $P_{\text {orb }}$ He-donors provide $\dot{M} \sim 10^{-8} M_{\odot} \mathrm{yr}^{-1}$. Correspondingly, Nelemans et al. [518, 519] considered "optimistic" and "pessimistic" scenarios for the formation of AM CVn stars in the He channel, depending on the amount of He that may accrete, detonate and destroy the AM CVn star precursor: $0.15 M_{\odot}$ or $0.30 M_{\odot}$. The difference in the predicted number of observed systems reached factor $\sim 2$ in favor of the first scenario. However, a comparison of the accretion and mass loss rates in Figures 25 and 14 suggests that the donors instead experience strong flashes of He-burning than detonations, and the model space density of AM CVn stars in $[518,519]$ is, most probably, an underestimate. This exacerbates the known problem of a possibly-almost factor $\sim 50$ deficit of the observed AM CVn star spatial density compared to theoretical predictions [651, 90]. However, as noted in [90], the "observational" estimate of the number of AM CVn stars depends on the assumed intrinsic distribution of their magnitudes, and uncertainty in this distribution may also contribute to the mismatch of theoretical expectations and observations.

An accurate knowledge of the formation channels and the space density of interacting doubledegenerates is necessary for a better understanding of the stability of mass exchange, processes in common envelopes and proper modeling of the gravitational wave foreground (Section 10). The same is true for ultra-compact X-ray sources. 


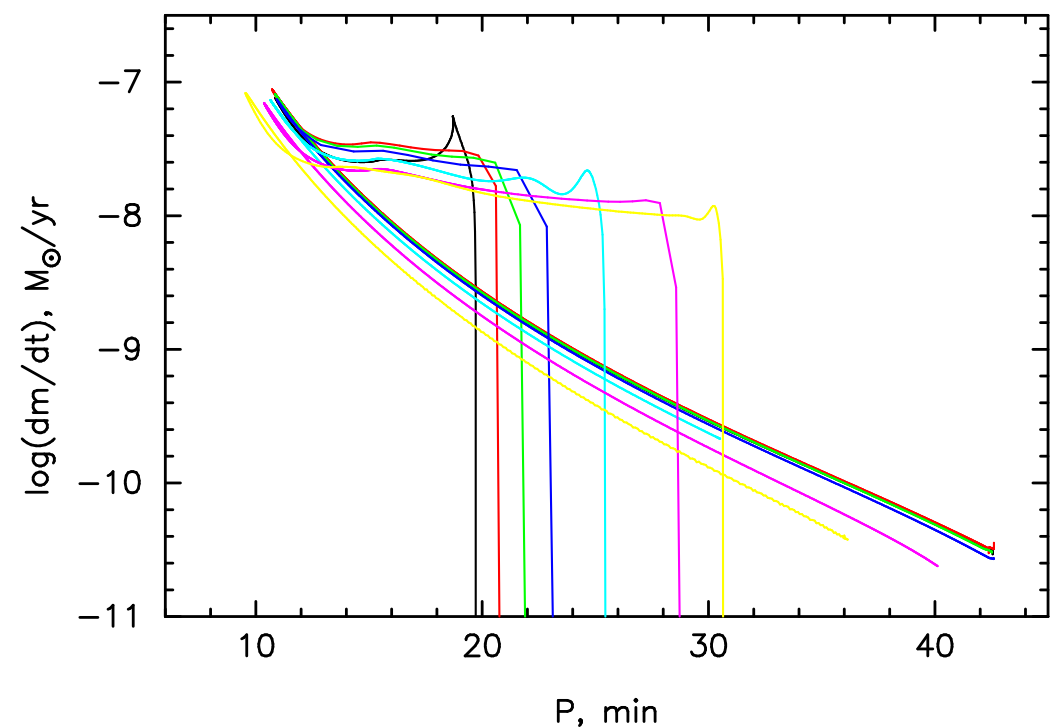

Figure 25: Mass-loss rate vs. orbital period dependence for semidetached systems with He-star donors and WD accretors, having post-common envelope $P_{\text {orb }}=20$ to $130 \mathrm{~min}$. Initial masses of components are $\left(M_{\mathrm{He}}+M_{\mathrm{WD}}\right)=(0.40+0.60) M_{\odot}$. He abundance in the cores of the donors at RLOF ranges from 0.98 to 0.066 (left to right). Image from [870], copyright by the author.

The chemical composition of IDD may reveal their origins [515, 521]. In the double degenerate channel, the matter transferred is a product of low-temperature CNO-burning, and the abundances of $\mathrm{He}, \mathrm{C}, \mathrm{N}$, and $\mathrm{O}$ depend on the WD progenitor's mass. In the He-star channel, the abundances depend on the initial mass of the donor and the extent of He-depletion by the time of RLOF (Figure 26). In the "evolved CV-channel", the matter may also contain the traces of hydrogen. As concerns UCXB, it was shown in [877] that the initial masses of their donors are limited from above by $\approx 0.45 M_{\odot}$. This means that some of the donors may descend from "hybrid" WDs. However, the latter lose their He-rich envelopes at very early phases of mass exchange, and, since they are descendants of helium stars, the matter transferred by them should not contain He. Nelemans et al. [521] constructed a set of diagnostic diagrams with abundance ratios (in mass) $X_{\mathrm{N}} / X_{\mathrm{He}}$, $X_{\mathrm{N}} / X_{\mathrm{C}}, X_{\mathrm{N}} / X_{\mathrm{O}}, X_{\mathrm{O}} / X_{\mathrm{He}}, X_{\mathrm{O}} / X_{\mathrm{C}}$ as functions of $P_{\mathrm{orb}}$ for different groups of donors. As an example, we show in Figure 27 such a diagram for the ratio $X_{\mathrm{N}} / X_{\mathrm{O}}$.

The above-described diagnostic of abundances in the spectra of known IDD and other means of analysis suggest that HM Cnc, CP Eri, GP Com, V396 Hya, SDSS J124058.03-015919.2, SDSS J080449.49+161624.8 almost definitely belong to the WD-family, while AM CVn, HP Lib, CR Boo, V803 Cen, SDSS J173047.59+554518.5 may have helium-star donors [521, 725, 88]. Significantly-evolved He-star donors are ruled out for SDSS J113732.32+405458.3 and SDSS J150551.58+065948.7 [91].

Among the UCXBs there are two systems (4U 1626-67 and 4U 0614+09) in which detection of $\mathrm{C}$ and $\mathrm{O}$ lines but no He lines suggests hybrid white dwarf or very evolved helium star donors. For $4 \mathrm{U}$ 1916-05, the detected He and N lines suggest a He white dwarf or an unevolved helium star donor [521]. For the rest, data are not sufficient for classification. 

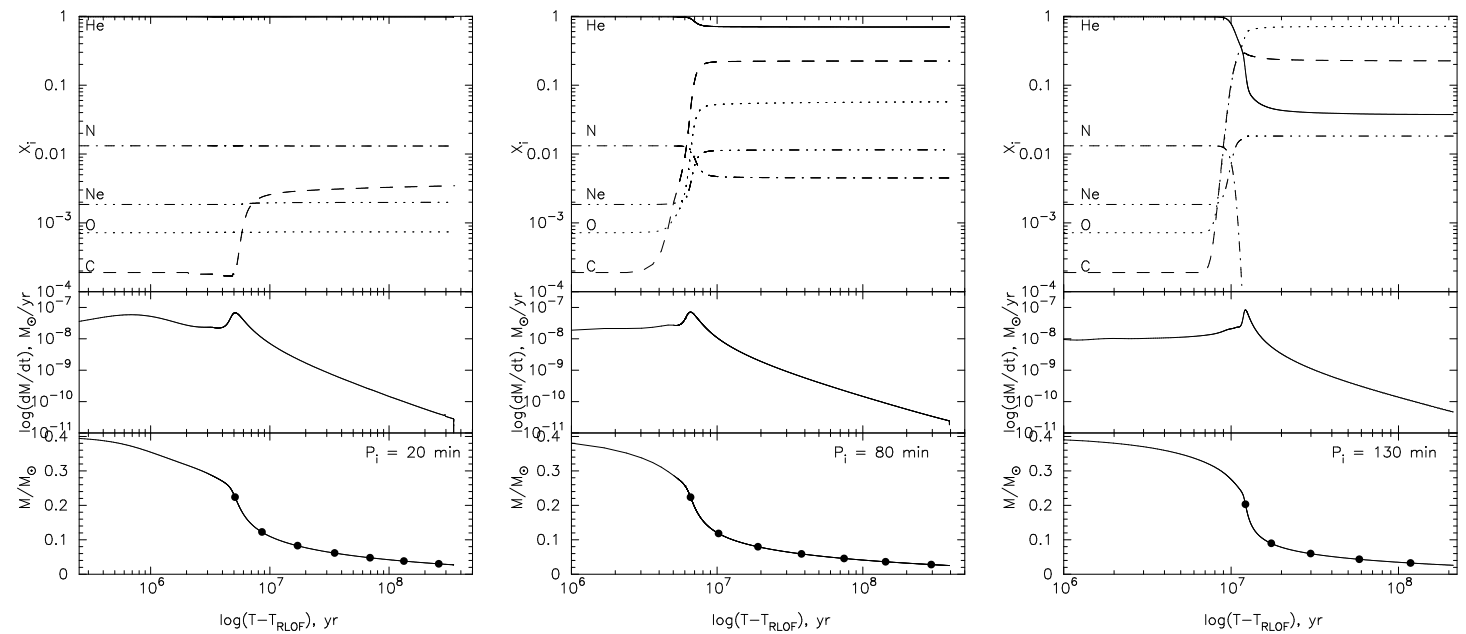

Figure 26: An overview of the evolution and chemical abundances in the transferred matter for helium star donors in ultra-compact binaries. We show abundances (top), mass-transfer rate (middle) and donor mass (bottom) as a function of time since the start of the Roche lobe overflow. The binary period is indicated by the solid circles in the bottom panels for $P_{\text {orb }}=15,20,25,30,35$, and $40 \mathrm{~min}$. The initial (post-common-envelope) orbital periods are indicated in the bottom panels. The sequences differ by the amount of nuclear processing before RLOF. Image reproduced with permission from Figure 4 of [521], copyright by the authors.

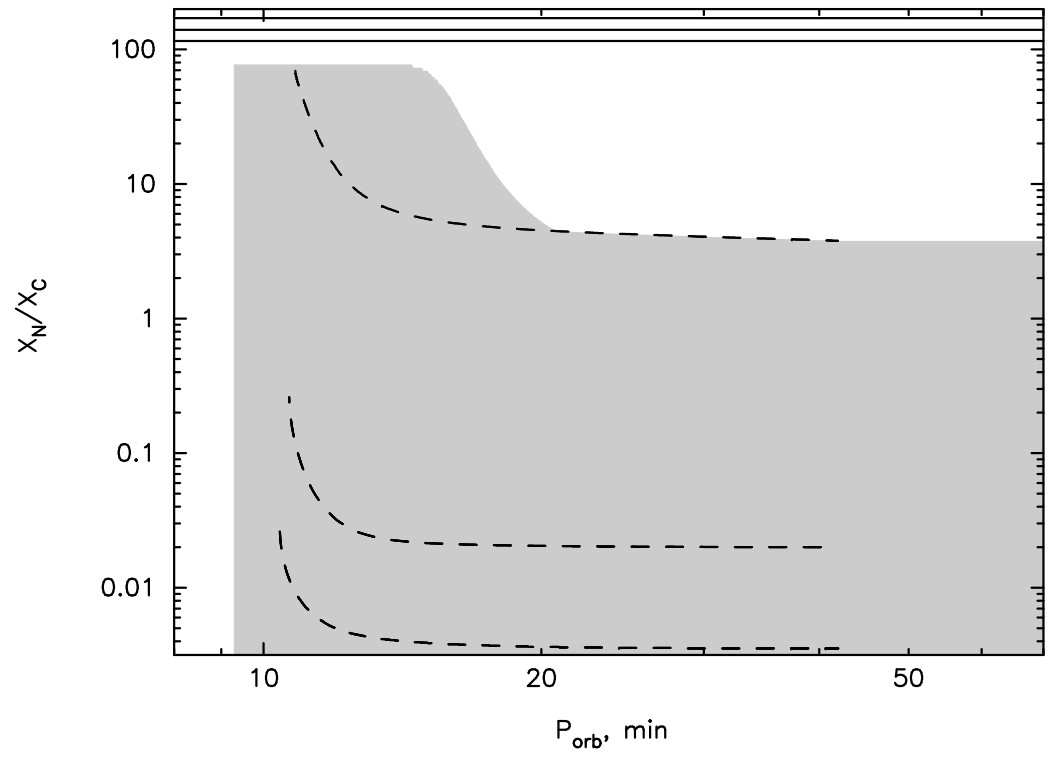

Figure 27: Abundance ratios (by mass) N/C for He-WD (the solid line) and He-star donors (the shaded region with dashed lines) as a function of the orbital period. For the helium star donors we indicate the upper part of the full range of abundances which extends to 0 . The dashed lines are examples of tracks shown in detail in Figure 26. The helium white dwarfs are descendants of 1, 1.5 and $2 M_{\odot}$ stars (top to bottom). Image reproduced with permission from Figure 11 of [521], copyright by the authors. 


\subsection{Final stages of evolution of interacting double-degenerate systems}

By means of population synthesis modeling, Solheim and Yungelson [727] found that accumulation of $M_{\mathrm{Ch}}$ by WD in the double-degenerate channel of formation and evolution of IDD is possible at a rate of $\sim 10^{-5} \mathrm{yr}^{-1}$, while Ruiter et al. [662] provided an estimate that is an order of magnitude higher. Note that in both studies SN Ia require massive WD accretors, which are not typical for observed IDD.

Yet another kind of explosive event may characterize the evolution of IDD [48, 697, 702] - faint thermonuclear supernovae dubbed SN .Ia.

As described above, in the WD-family of AM CVn stars, the mass transfer rate initially is as high as $\sim(1-3) \times 10^{-6} M_{\odot} \mathrm{yr}^{-1}$ and decreases at later times. While during the initial phase of accretion the nuclear burning in the accreted He envelope of the WD is thermally stable, it becomes thermally unstable as $\dot{M}_{\text {accr }}$ drops (Figure 14). In the He-star family, the mass transfer rate before reaching the orbital period minimum is several $10^{-8} M_{\odot} \mathrm{yr}^{-1}$, i.e., accretors are in the strong He-flashes regime ab ovo. The "ignition" mass for He-outbursts increases with decreasing $\dot{M}_{\text {accr }}$. This yields $\sim 10$ He-flashes in the $\sim\left(10^{6}-10^{7}\right)$ yr before the amount of accreted mass required to achieve He-ignition $(\sim 0.1) M_{\odot}$ becomes larger than the mass of the donor (Figure 28), while de facto the binaries are already in the "detonation" regime of He-burning (Figure 14). Thus, there must be the "last" most powerful outburst with an ignition mass of $\sim 0.1 M_{\odot}$ which can occur in the hydrodynamic regime. It can lead to He-detonation, which can be potentially observable as a faint thermonuclear supernova [48]. Since the mass transfer rate drops very rapidly after RLOF, it is plausible that the observed AM CVn stars already experienced their last strong He-flash (which could be of the SN .Ia scale) and are now slowly transferring He without any expected nuclearpowered phenomena. Supernovae .Ia probably do not initiate detonations of low-mass accretors of AM CVn stars since the masses of the exploding shells are insufficient for this [713, 698].

As estimated in [48], SN .Ia may happen every 5000 to $15000 \mathrm{yr}$ in an E/S0 galaxy. Their brightness and duration are about $1 / 10$ of the ordinary SN Ia event. The rise times of the light curves in all bands are very rapid $(<10$ days). The explosion nucleosynthesis in these events primarily yields heavy $\alpha$-chain elements $\left({ }^{40} \mathrm{Ca}\right.$ through ${ }^{56} \mathrm{Ni}$ ) and unburned $\mathrm{He}$ (up to 80\%) [702, 493]. It is interesting that with the 1-day dynamic-cadence experiment on the Palomar Transient Factory ${ }^{31}$ it is expected to annually discover a few such events.

SN 2010X (PTF 10bhp) with extremely rapid decay ( $\tau=5$ day) was, for instance, suggested as a candidate SN .Ia [344]. Another candidate may be a faint type Ib supernova, SN 2005E, in the halo of the nearby isolated galaxy, NGC 1032. Spectroscopic observations and analysis reveal high velocities of the ejecta, dominated by helium-burning products including a large amount of radioactive ${ }^{44} \mathrm{Ti}$, just as expected for SN .Ia. However, as noticed by Drout et al. [165], e.g., severely stripped-envelope SN Ic explosions may mimic SNe .Ia.

It is possible that strong flashes of He-burning can be identified with He-novae, like H-outbursts are identified with classical novae. The sole known object that may be a prototype of He-novae - V445 Pup erupted in 2000, and its optical and IR spectra are hydrogen-deficient [15, 853]. Provisional $P_{\text {orb }}$ of V445 Pup is $\approx 0.65$ day [247]. Several other candidate He-novae have been suggested, but remain still unexplored [657]. The estimated pre-outburst luminosity of V445 Pup is $\log \left(L / L_{\odot}\right)=4.34 \pm 0.36$ [854]. Apparently, it is associated with a more massive system than the typical AM CVn star, but one cannot exclude nova-scale events among AM CVn's.

${ }^{31}$ http://www.astro.caltech.edu/ptf 


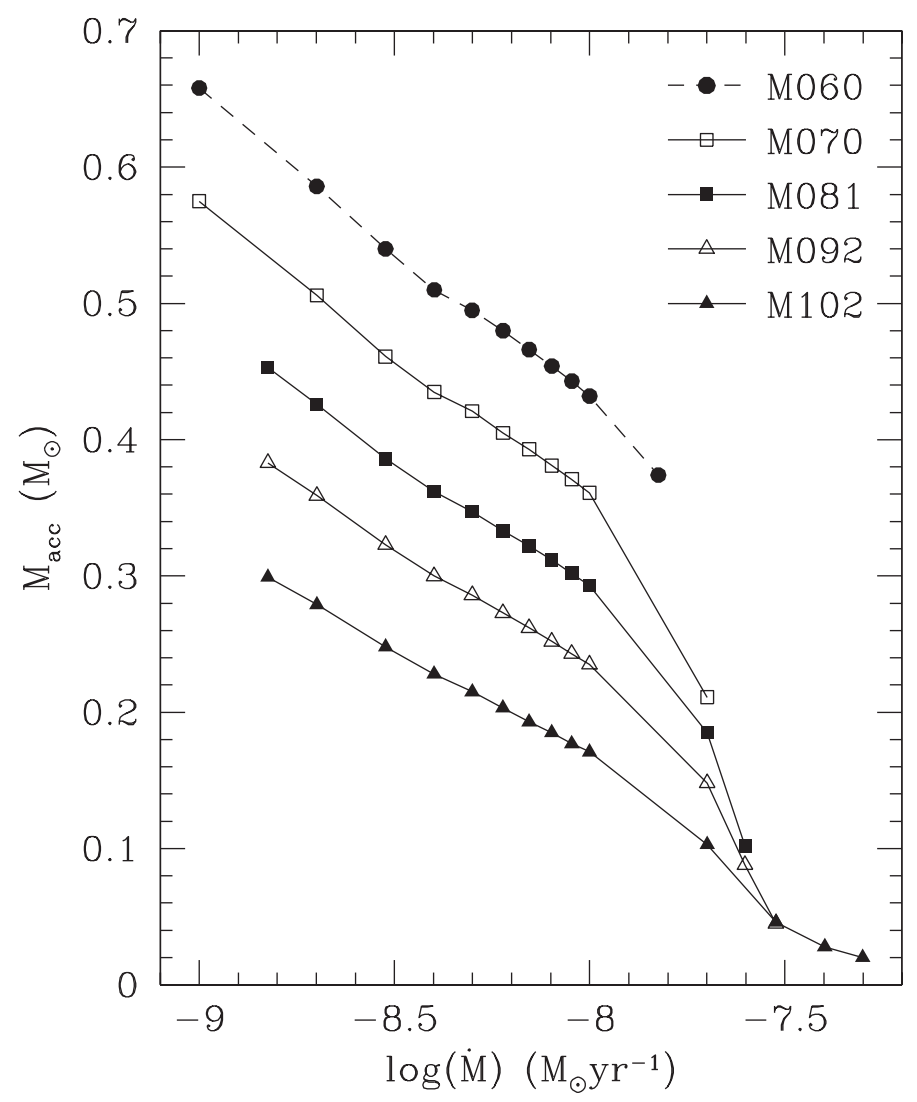

Figure 28: Accreted mass as a function of the accretion rate for models experiencing a dynamical Heflash. The lines refer to WD with different initial masses - 0.6, 0.7, 0.81, 0.92, $1.02 M_{\odot}$. Piersanti, Tornambé and Yungelson in prep.

\section{Gravitational Waves from Compact Binaries with White- Dwarf Components}

We need to start this section with a caveat that until now virtually all studies on the detection of low-frequency GWR from compact binaries, with the single exception of [531], have been made with the future launch of LISA in mind. At the time of writing only several months have elapsed since the decision to cancel LISA and the introduction of the eLISA project. Therefore, almost all estimates involving sensitivity of the detector are still "LISA-oriented" and only in some cases corrections for the reduced (by about an order of magnitude) eLISA sensitivity are available. Nevertheless, as the physics behind GWR emission is unchanged, we present in this section, as examples, "LISA-oriented" results, unless stated otherwise.

It was suggested initially that contact W UMa binaries will dominate the Galactic gravitationalwave background at low frequencies [487]. However, it was shown in [784, 467, 180, 426, 282] that it will, most probably, be totally dominated by detached and semidetached WD binaries.

As soon as it was recognized that the birth rate of Galactic close WD binaries may be rather high, and even before detection of the first close detached DD was reported in 1988 [665], in 1987 Evans, Iben, and Smarr [180] accomplished an analytical study of the detectability of the signal from the Galactic ensemble of DDs, assuming certain average parameters for DDs. Their main 
findings can be formulated as follows. Let us assume that there exists a certain distribution of DDs over frequency of the signal $f$ and the strain amplitude $h: n(f, h)$. The weakest signal is $h_{\mathrm{w}}$. For the time span of observations $\tau_{\text {int }}$, the frequency resolution bin of the detector is $\Delta f_{\text {int }} \approx 1 / \tau_{\text {int }}$. Then, integration of $n(f, h)$ over amplitude down to a certain limiting $h$ and over $\Delta f$ gives the mean number of sources per unit frequency resolution bin for a volume defined by $h$. If for a certain $h_{n}$

$$
\int_{h_{n}}^{\infty}\left(\frac{d n}{d f d h}\right) \Delta \nu_{\mathrm{int}}=1
$$

then all sources with $h_{n}>h>h_{\mathrm{w}}$ overlap. If in a certain resolution bin $h_{n}>h_{\mathrm{w}}$ does not exist, individual sources may be resolved in this bin for a given integration time (if they are above the detector's noise level). In the bins where binaries overlap, they produce "confusion noise": an incoherent sum of signals; the frequency above which the resolution of individual sources becomes possible was referred to as the "confusion limit", $f_{\mathrm{c}}$. Evans et al. found $f_{\mathrm{c}} \approx 10 \mathrm{mHz}$ and $3 \mathrm{mHz}$ for integration times $10^{6} \mathrm{~s}$ and $10^{8} \mathrm{~s}$, respectively.

Independently, the effect of confusion of Galactic binaries was demonstrated by Lipunov, Postnov, and Prokhorov [427], who used simple analytical estimates of the GW confusion limited signal produced by unresolved binaries whose evolution is driven by GWs only; in this approximation, the expected level of the signal depends solely on the Galactic merger rate of WD binaries (see [252] for more details). Later, analytic studies of the GW signal produced by stellar binaries at low frequencies were continued in [187, 282, 608, 281]. A more detailed approach to the estimate of the GW foreground is possible using population synthesis models [426, 828, 518, 519, 661, 864, 865]. Convenient analytical expressions allowing us to compare the results of different studies as a function of the assumed Galactic model and merger rate of dwarf binaries are provided by Nissanke et al. [531].

Note that in early studies the combined signal of Galactic binaries was dubbed "background" and considered as a "noise". However, Farmer and Phinney [188] stressed that this signal will in fact be a "foreground" for "backgrounds" produced in the early universe. But only circa 20082009 the term "foreground" became common in discussions of Galactic binaries. As summarized by Amaro-Seoane et al. [10], the overall level of the foreground is a measure of the total number of ultra-compact binaries; the spectral shape of the foreground contains information about the homogeneity of the sample, as models with specific types of binaries predict a very distinct shape; the geometrical distribution of the sources can be found by a probe like eLISA.

Giampieri and Polnarev [240], Farmer and Phinney [188] and Edlund et al. [170] showed that, due to the concentration of sources in the Galactic center and the inhomogeneity of the LISAantenna pattern, the foreground should be strongly modulated in a year of observations (Figure 29), with time periods in which the foreground is by more than a factor two lower than during other periods. This is not the case for the signal from extra-galactic binaries, which should be almost isotropic. The characteristics of the modulation can provide information on the distribution of the sources in the Galaxy, as the different Galactic components (thin disk, thick disk, halo) contribute differently to the modulation. Additionally, during "low signal" periods, antenna will be able to observe objects that are away from the Galactic plane.

Referring the reader for comprehensive review of eLISA science to [10], we briefly note that measurements of individual binaries provide the following information. If the system is detached, the evolution of the signal is dominated by gravitational radiation and, in principle, three variables may be measured:

$$
h \propto \mathcal{M}^{5 / 3} f^{2 / 3} D^{-1}, \quad \dot{f} \propto \mathcal{M}^{5 / 3} f^{11 / 3}, \quad \ddot{f}=\frac{11}{3} \frac{\dot{f}}{f},
$$

where $h$ is the strain amplitude, $f$ is the frequency, $\mathcal{M}=\left(m_{1} m_{2}\right)^{3 / 5} /\left(m_{1}+m_{2}\right)^{1 / 5}$ is the chirp mass, and $D$ is the distance to the source. Thus, the determination of $h, f$, and $\dot{f}$ (which is expected 


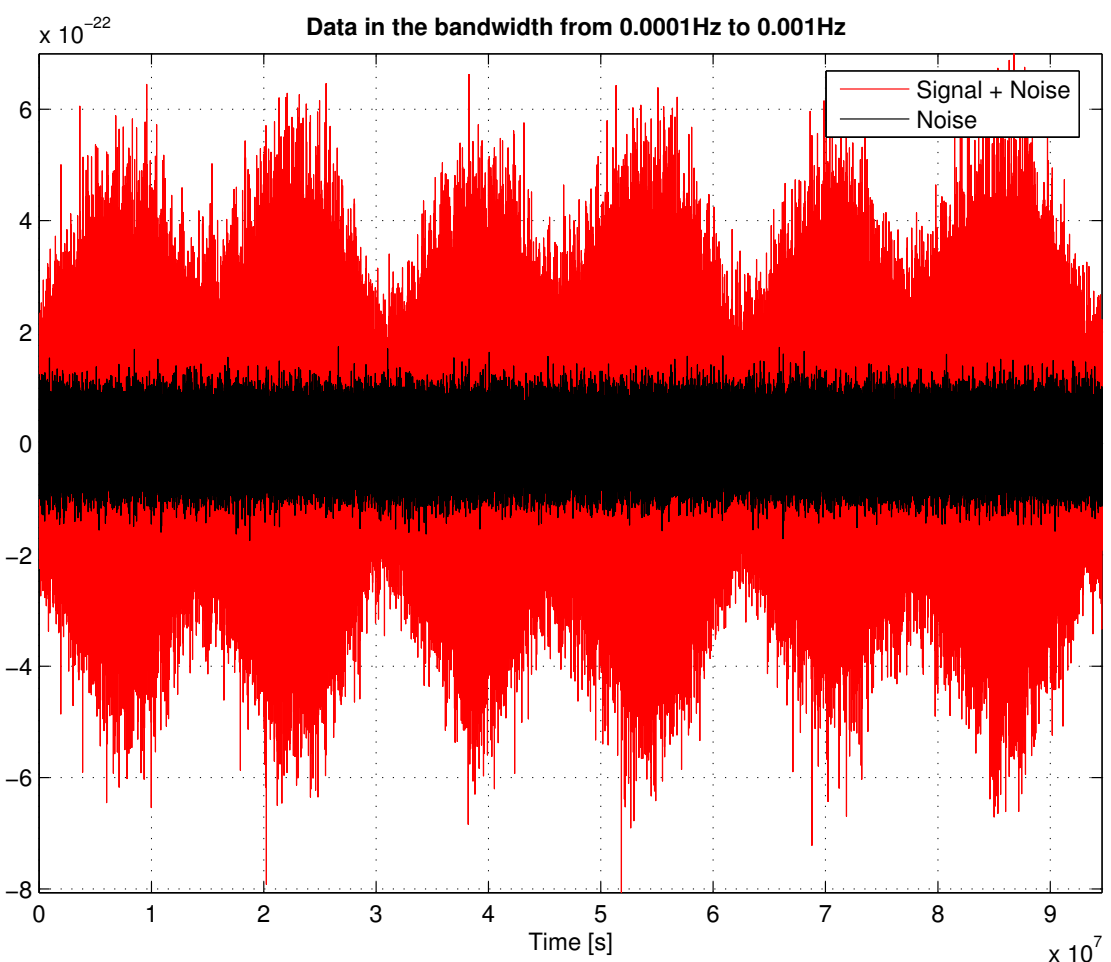

Figure 29: Simulated time-series of the DD Galactic foreground signal of 3 years of data. "Noise" is the instrumental LISA noise. Based on computations in [519]. Image reproduced with permission from [170], copyright by ASP.

to be possible for $25 \%$ of eLISA sources [10]) provides $\mathcal{M}$ and $D$. The value of $\ddot{f}$ (which may be possible for a few high-SNR systems) gives information on deviations from the "pure" GW signal due to tidal effects and mass-transfer interactions. An estimate of the effects of tidal interaction is especially interesting, since it is expected that this effect will be typical for pre-merger systems in which it may lead to strong heating, raising the luminosity of each component to $\simeq 20 L_{\odot}$, which gives a chance of optical detection on the time scale of a human life; moreover, it can even affect conditions for thermonuclear burning in the inner and outer layers of WD [829, 304, 827, 218, 219].

Nelemans et al. [518] constructed a population synthesis model of the gravitational-wave signal from the Galactic disk population of binaries containing two compact objects. The model included detached DDs, semidetached DDs, ${ }^{32}$ detached systems of NSs and BHs with WD companions, NS and BH binaries. For details of the model we refer the reader to the original paper and references therein. Table 8 shows a number of systems with different combinations of components in the Nelemans et al. model. ${ }^{33}$ Note that these numbers strongly depend on assumptions in the population synthesis code, especially on the normalization of the stellar birth rate, star formation history, distributions of binaries over initial masses of components, their mass-ratios and orbital separations, the treatment of stellar evolution, common envelope formalism, etc. For binaries with relativistic components (i.e., descending from massive stars) an additional uncertainty is brought in

32 The "optimistic" model of the population of IDD was taken [512], which assumes effective tidal interaction and inefficient destruction of hypothetical progenitors of AM CVn stars due to double-degenerate SN Ia. Thus, the numbers provided in the table are upper limits.

33 These numbers, as for models discussed below, represent one random realization of the model and are subject to Poissonian noise. 
Table 8: Current birth rates and merger rates per year for Galactic disk binaries containing two compact objects and their total number in the Galactic disk [518].

\begin{tabular}{lccc}
\hline Type & Birth rate & Merger rate & Number \\
\hline Detached DD & $2.5 \times 10^{-2}$ & $1.1 \times 10^{-2}$ & $1.1 \times 10^{8}$ \\
Semidetached DD & $3.3 \times 10^{-3}$ & - & $4.2 \times 10^{7}$ \\
NS + WD & $2.4 \times 10^{-4}$ & $1.4 \times 10^{-4}$ & $2.2 \times 10^{6}$ \\
NS + NS & $5.7 \times 10^{-5}$ & $2.4 \times 10^{-5}$ & $7.5 \times 10^{5}$ \\
BH + WD & $8.2 \times 10^{-5}$ & $1.9 \times 10^{-6}$ & $1.4 \times 10^{6}$ \\
BH + NS & $2.6 \times 10^{-5}$ & $2.9 \times 10^{-6}$ & $4.7 \times 10^{5}$ \\
BH + BH & $1.6 \times 10^{-4}$ & - & $2.8 \times 10^{6}$ \\
\hline
\end{tabular}

by assumptions on stellar wind mass loss and natal kicks. The factor of uncertainty in the estimated number of systems of a specific type may easily exceed 10 (cf. [265, 518, 791, 294, 519, 661, 864]). Thus, these numbers should be taken with some caution.

Table 8 immediately shows that detached DDs, as expected, dominate the population of compact binaries, as found by other authors, as well. While Table 8 gives total numbers, examples of contributions from different formation channels and spatial components of the Galaxy may be found, e.g., in [661, 864, 865], with a caveat that models in these paper are different from the model in [518]. Population-synthesis studies confirmed the expectation that a contribution to the signal in the LISA/eLISA frequency range from thick disk, halo, and bulge binaries should not be significant, since the binary systems there are predominantly old and either merged or evolved to too long periods as IDD [660, 864, 661]. It was also shown that the contribution from extra-galactic binaries to the signal in the LISA/eLISA range will be $\sim 1 \%$ of the foreground [188]. The list of models computed before 2012 and an algorithm allowing one to compare the results of different calculations to the extent of their dependence on Galactic model and normalization may be found in [531].

Population synthesis computations yield the ensemble of Galactic binaries at a given epoch with their specific parameters $M_{1}, M_{2}$, and $a$ and the Galactic location. Figure 30 shows examples of the relationship between the frequency of emitted radiation and amplitude of the signals from the "typical" double degenerate system that evolves into contact and merges, for an initially detached double degenerate system that stably exchanges matter after the contact, i.e., an AM CVn-type star and its progenitor, and for an UCXB and its progenitor. For the AM CVn system effective spinorbital coupling is assumed [512,464], Figure 22. The difference in the properties and evolution of DD and IDD (merger vs. bounce and continuation of evolution with decreasing mass) is reflected in the distributions and magnitude of their strains shown in Figure 31 [170].

Note that there is a peculiar difference between WD pairs that merge and pairs that experience a stable mass exchange. The pairs that coalesce stop emitting GWs on a relatively short time-scale (on the order of the period of the last stable orbit, typically a few minutes) [443]. Thus, if we would be lucky to observe a chirping WD and a sudden disappearance of the signal, this will manifest a merger. However, the chance to detect such an event is small since the Galactic rate of merging WD binaries is $\sim 10^{-2} \mathrm{yr}^{-1}$ only.

For the system with a NS, the mass exchange rate is limited by the critical Eddington value, and excess of matter is "re-ejected" from the system (see Section 3.3.3 and [877]). Note that for an AM CVn-type star it takes only $\sim 300$ Myr after contact to evolve to $\log f=-3$, which explains their accumulation at lower $f$. For UCXBs this time interval is only $\sim 20 \mathrm{Myr}$. 


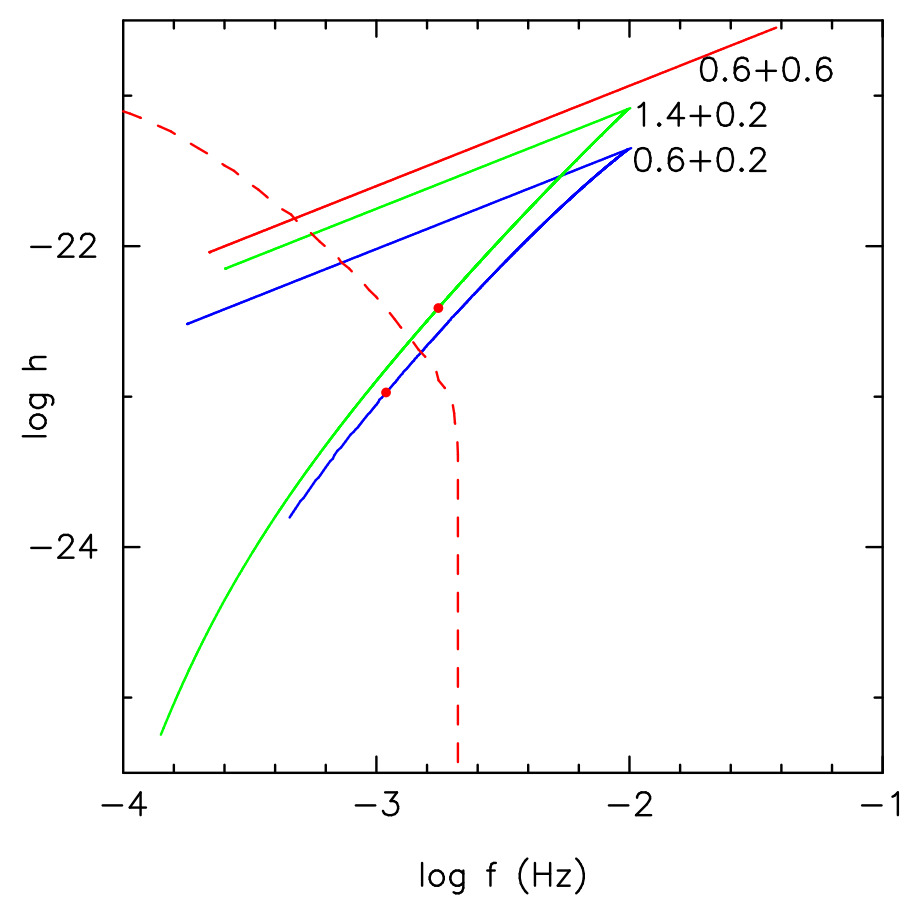

Figure 30: Dependence of the dimensionless strain amplitude for a WD + WD detached system with initial masses of the components of $0.6 M_{\odot}+0.6 M_{\odot}$ (red line), a WD + WD system with $0.6 M_{\odot}+0.2 M_{\odot}$ (blue line) and a NS + WD system with 1.4 $M_{\odot}+0.2 M_{\odot}$ (green line). All systems have an initial separation of components $1 R_{\odot}$ and are assumed to be at a distance of $1 \mathrm{Kpc}$ (i.e., the actual strength of the signal has to be scaled with factor $1 / d$, with $d$ in $\mathrm{Kpc}$ ). For the DD system, the line shows an evolution into contact, while for the other two systems the upper branches show pre-contact evolution and lower branches - a post-contact evolution with mass exchange. The total time-span of evolution covered by the tracks is 13.5 Gyr. Red dots mark the positions of systems with components' mass ratio $q=0.02$, below which the conventional picture of evolution with a mass exchange may be not valid. The red dashed line marks the position of the confusion limit as determined in [519].

In the model discussed, the systems are distributed randomly in the Galactic disk according to

$$
\rho(R, z)=\rho_{0} e^{-R / H} \operatorname{sech}(z / \beta)^{2} \mathrm{pc}^{-3},
$$

where $H=2.5 \mathrm{kpc}[664]$ and $\beta=200 \mathrm{pc}$. The Sun is located at $R_{\odot}=8.5 \mathrm{kpc}$ and $z_{\odot}=-30 \mathrm{pc}$. Then it is possible to compute the strain amplitude for each system. The power spectrum of the signal from the population of binaries as it would be detected by a gravitational wave detector, may be simulated by computation of the distribution of binaries over $\Delta f=1 / T$ wide bins, with $T$ being the total integration time. Figure 32 shows the resulting confusion limited foreground signal. In Figure 33 the number of systems per bin is plotted. The assumed integration time is $T=1$ yr. Semidetached WD binaries, which are less numerous than their detached cousins and have lower strain amplitude, dominate the number of systems per bin in the frequency interval $-3.4 \lesssim \log f(\mathrm{~Hz}) \lesssim-3.0$ producing a peak there, see also Figure 33 .

Results presented below may be, to some extent, considered as an illustration of the signal detected by a space-born detector, since they are model-dependent and "LISA-oriented", but they show, at least qualitatively, the features of the signal detected by any space-based GW antenna.

Figure 32 shows that there are many systems with a signal amplitude much higher than the average in the bins with $f<f_{\mathrm{c}}$, suggesting that even in the frequency range seized by the confusion 

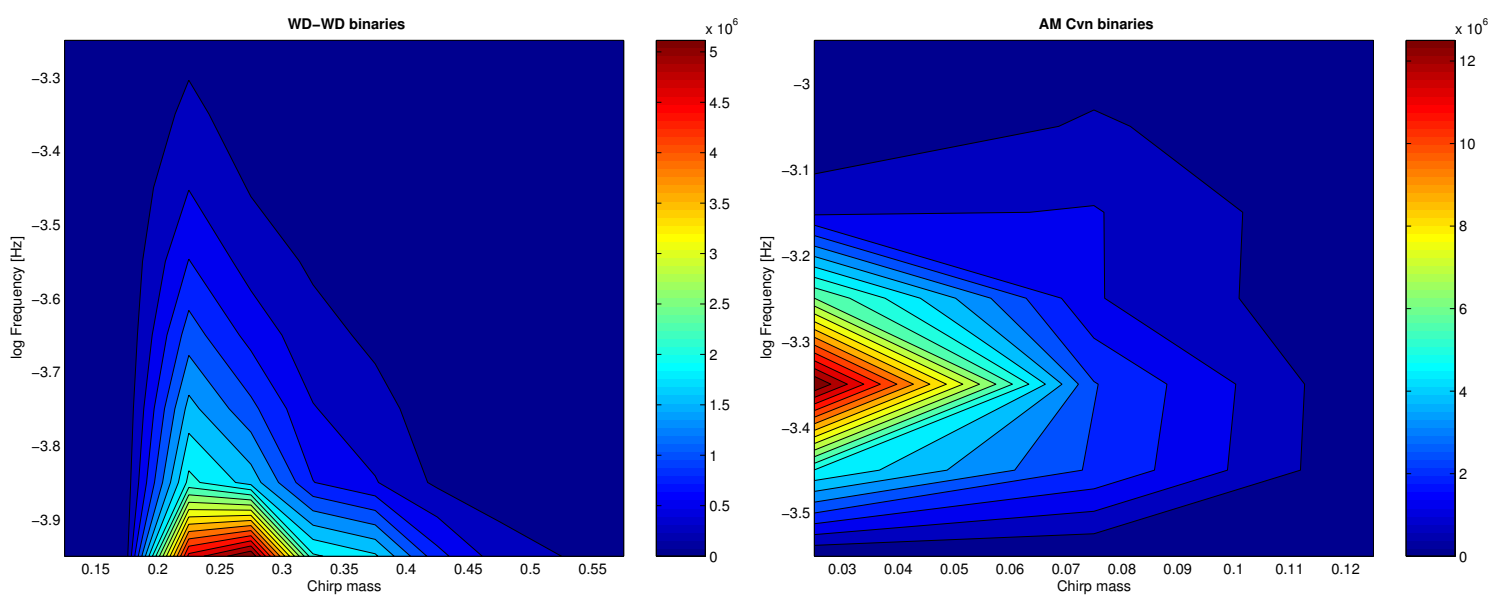

Figure 31: The distribution of Galactic detached WD + WD binaries and interacting WD (AM CVn stars) as a function of the gravitational wave frequency and chirp mass. From [170], based on computations in [519].

noise some systems can be detectable above the noise level. The latter will affect the ability to detect extra-galactic massive black-hole binaries (Figure 2) and to derive their parameters.

Population synthesis also shows that the notion of a unique "confusion limit" is an artefact of the assumption of a continuous distribution of systems over their parameters. For a discrete population of sources it appears that for a given integration time there is actually a range of frequencies where there are both empty resolution bins and bins containing more than one system (see Figure 34). For this "statistical" notion of $f_{\mathrm{c}}$, Nelemans et al. [518] get the first bin containing exactly one system at $\log f_{\text {res }}(\mathrm{Hz}) \approx-2.84$, while up to $\log f(\mathrm{~Hz}) \approx-2.3$ there are bins containing more than one system. Other authors find similar, but slightly more conservative limits on $\log f_{\text {res }}$ : -2.25 [864], -2.44 [828], -2.52 [661].

Nelemans et al. [518] also noted the following point. Previous studies of GW emission from the AM CVn systems have found that they hardly contribute to the GW foreground, despite that at $f \approx(0.3-1.0) \mathrm{mHz}$ they outnumber the detached DDs. This happens because at these $f$ their chirp mass $\mathcal{M}$ is much smaller than that of a typical detached system. However, it was overlooked before that at higher frequencies, where the number of AM CVn systems is much smaller, their $\mathcal{M}$ is similar to that of the detached systems from which they descend. This is also confirmed by the latest studies of GW foreground by Nissanke et al., see Figure 36.

In $[518,519]$ the following objects were considered as resolved: single source per frequency bin with signal-to-instrument noise ratio (SNR) $\geq 1$ for sources that can be resolved above the confusion limit $f_{\mathrm{c}}$ or SNR $\geq 5$ for systems with $f<f_{\mathrm{c}}$ that are detectable above the noise level. This resulted in approximately equal numbers of resolvable detached double degenerates and interacting double degenerates - about 11000 objects of every kind.

However, the above-mentioned criterion for resolution may be oversimplified. A more rigorous approach implies detection using an iterative identify-and-subtract process, where the signal-tonoise ratio of a source is evaluated with respect to noise from both the instrument and the partiallysubtracted foreground $[763,531]$. This procedure was applied by Nissanke et al. [531] to the same sample of binaries as in [518, 519], for both "optimistic" (Case 1 below) and "pessimistic" (Case 2) scenarios (see Sections 7 and 9) with different efficiency of the formation of double degenerates.

Figure 36 shows the frequency-space density and GW foreground of WD and AM CVn binary systems for these two scenarios, as computed in [531]. Qualitatively the results are in good agree- 


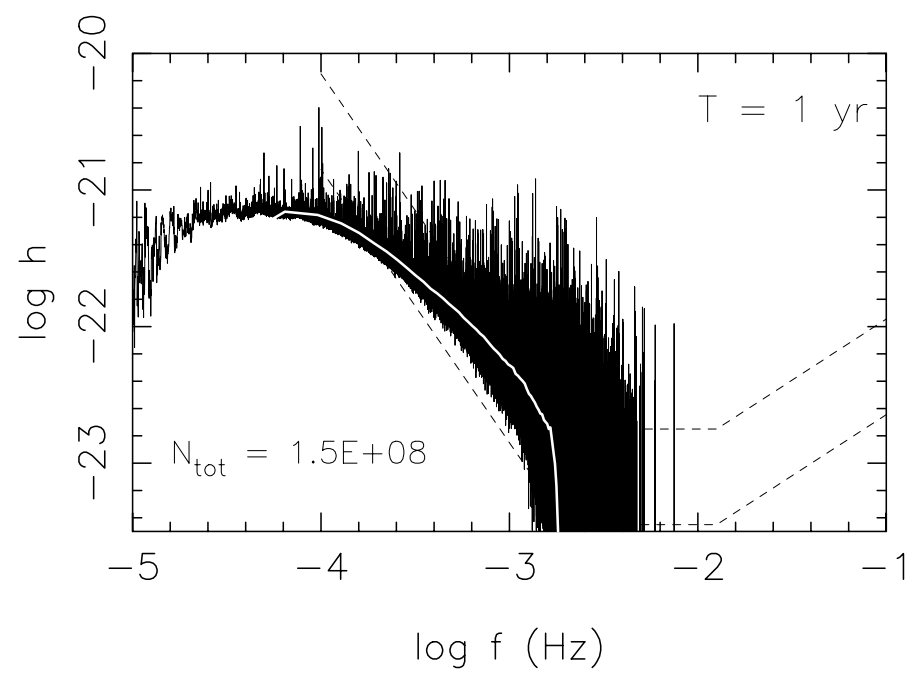

Figure 32: GWR foreground produced by detached and semidetached WD binaries as it was expected to be detected by LISA. The assumed integration time is $1 \mathrm{yr}$. The 'noisy' black line gives the total power spectrum, the white line shows the average. The dashed lines show the expected LISA sensitivity for a $S / N$ of 1 and 5 [408]. Semidetached WD binaries contribute to the peak between $\log f \simeq-3.4$ and -3.0 . Image reproduced with permission from [518], copyright by ESO.

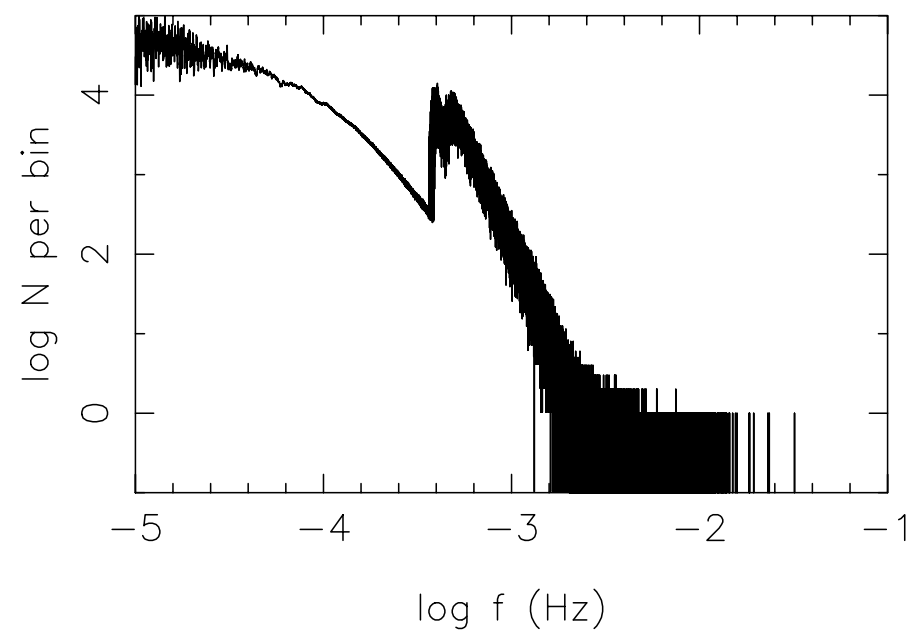

Figure 33: The number of systems per bin on a logarithmic scale. Semidetached WD binaries contribute to the peak between $\log f \simeq-3.4$ and -3.0 . Image reproduced with permission from [518], copyright by ESO. 


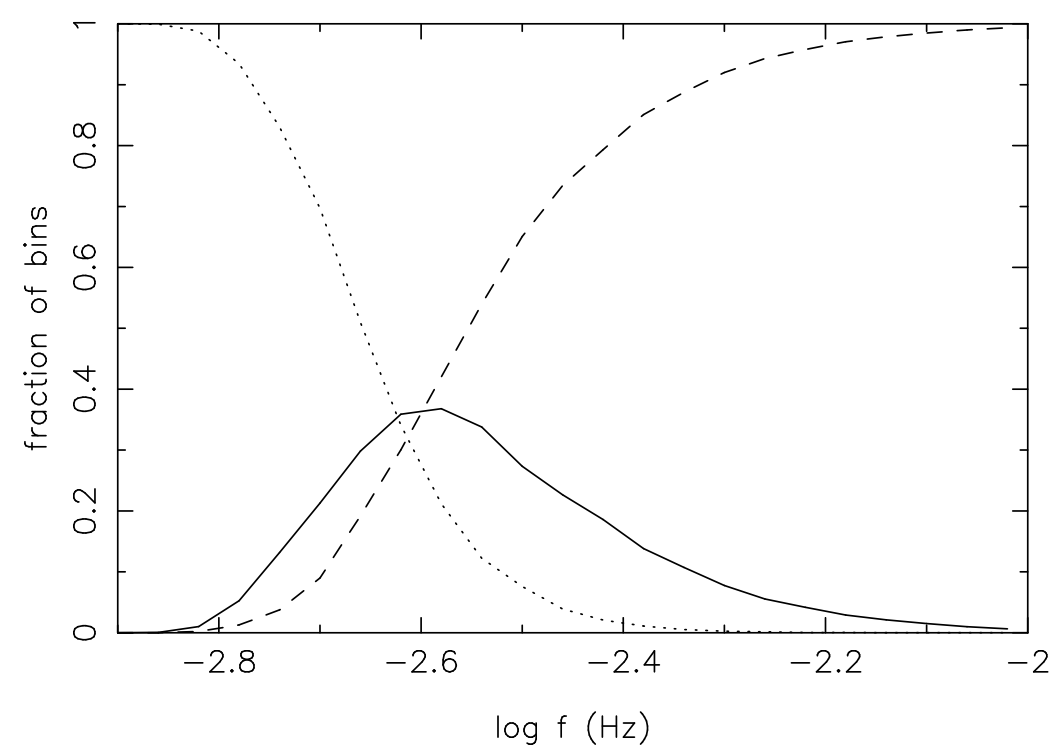

Figure 34: Fraction of bins that contain exactly one system (solid line), empty bins (dashed line), and bins that contain more than one system (dotted line) as a function of the signal frequency. From [518], copyright by ESO.

Table 9: Detectable sources (5 Mkm detector - two upper rows, $1 \mathrm{Mkm}$ detector - two lower rows). Values are shown for $\mathrm{SNR}_{\mathrm{thr}}=5$, one interferometric observable, and one year of observation, with approximate scalings as a function of $\rho_{\text {eff }}=\operatorname{SNR}_{\mathrm{thr}}\left(T_{\mathrm{obs}} / \mathrm{yr}\right)^{-1 / 2}\left(N_{\mathrm{obs}}\right)^{-1 / 2}[531]$.

\begin{tabular}{crrr}
\hline & DD & AM CVn $(D D)$ & AM CVn $(H e-W D)$ \\
\hline case 1 & $11,898 \times\left(\rho_{\text {eff }} / 5\right)^{-1.4}$ & $2,093 \times\left(\rho_{\text {eff }} / 5\right)^{-1.8}$ & $122 \times\left(\rho_{\text {eff }} / 5\right)^{-2.8}$ \\
case 2 & $13,957 \times\left(\rho_{\text {eff }} / 5\right)^{-1.4}$ & $16 \times\left(\rho_{\text {eff }} / 5\right)^{-1.7}$ & $72 \times\left(\rho_{\text {eff }} / 5\right)^{-2.9}$ \\
\hline case 1 & $6,034 \times\left(\rho_{\text {eff }} / 5\right)^{-1.2}$ & $719 \times\left(\rho_{\text {eff }} / 5\right)^{-1.4}$ & $38 \times\left(\rho_{\text {eff }} / 5\right)^{-2.6}$ \\
case 2 & $6,337 \times\left(\rho_{\text {eff }} / 5\right)^{-1.2}$ & $4 \times\left(\rho_{\text {eff }} / 5\right)^{-1.4}$ & $25 \times\left(\rho_{\text {eff }} / 5\right)^{-2.2}$ \\
\hline
\end{tabular}

ment with $[518,519]$, despite the fact that the predicted number of systems is lower by a factor of five.

The frequency-space density and GW foreground of DD and AM CVn systems for two scenarios are shown in Figure 37 and appropriate numbers of systems are presented in Table 9.

The estimates were made for two assumed arm-lengths of the detector. The number of detected sources and the level of residual confusion foreground may be scaled by power laws of a single "effective-SNR parameter" $\rho_{\text {eff }}=\operatorname{SNR}_{\text {thr }}\left(T_{\text {obs }} / \mathrm{yr}\right)^{-1 / 2}\left(N_{\text {obs }}\right)^{-1 / 2}$, where $\operatorname{SNR}_{\text {thr }}$ is the detection threshold, $T_{\mathrm{obs}}$ is the time of observations, and $N_{\text {obs }}$ is the number of Time-Delayed Interferometry observables (TDI - a specific method to properly time-shift and linearly combine independent Doppler measurements, that is necessary in the case of unequal arm-lengths of the detector [150]; $N_{\text {obs }}=1$ for a two-arms configuration of the detector).

For the LISA-like configuration, the number of detected DDs is comparable to earlier estimates for the "optimistic" case of $[518,519]: \simeq 11000$. The number of AM CVn stars that was comparable to the number of DDs, now strongly drops due to a higher required SNR defined with respect to 


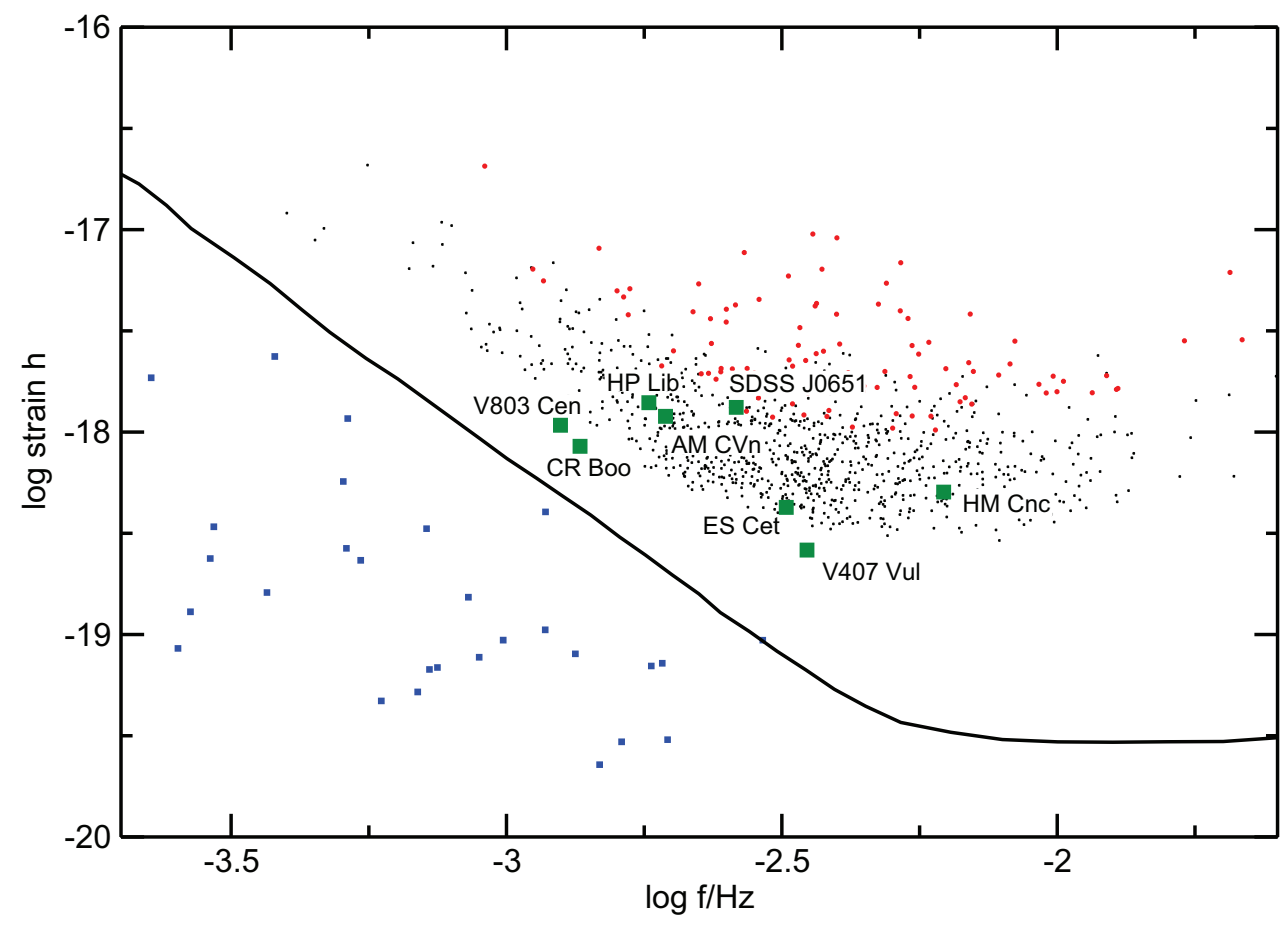

Figure 35: Strain amplitude spectral density (in $\mathrm{Hz}^{-1 / 2}$ ) versus frequency for the verification binaries and the brightest binaries in the simulated Galactic population of ultra-compact binaries [519]. The solid line shows the sensitivity of eLISA. The assumed integration time is 2 yrs. 100 simulated binaries with the largest strain amplitude are shown as red squares. Observed ultra-compact binaries are shown as blue squares, while the subsample of them that can serve as verification binaries is marked as green squares. Image reproduced with permission from [11], copyright by the authors. 


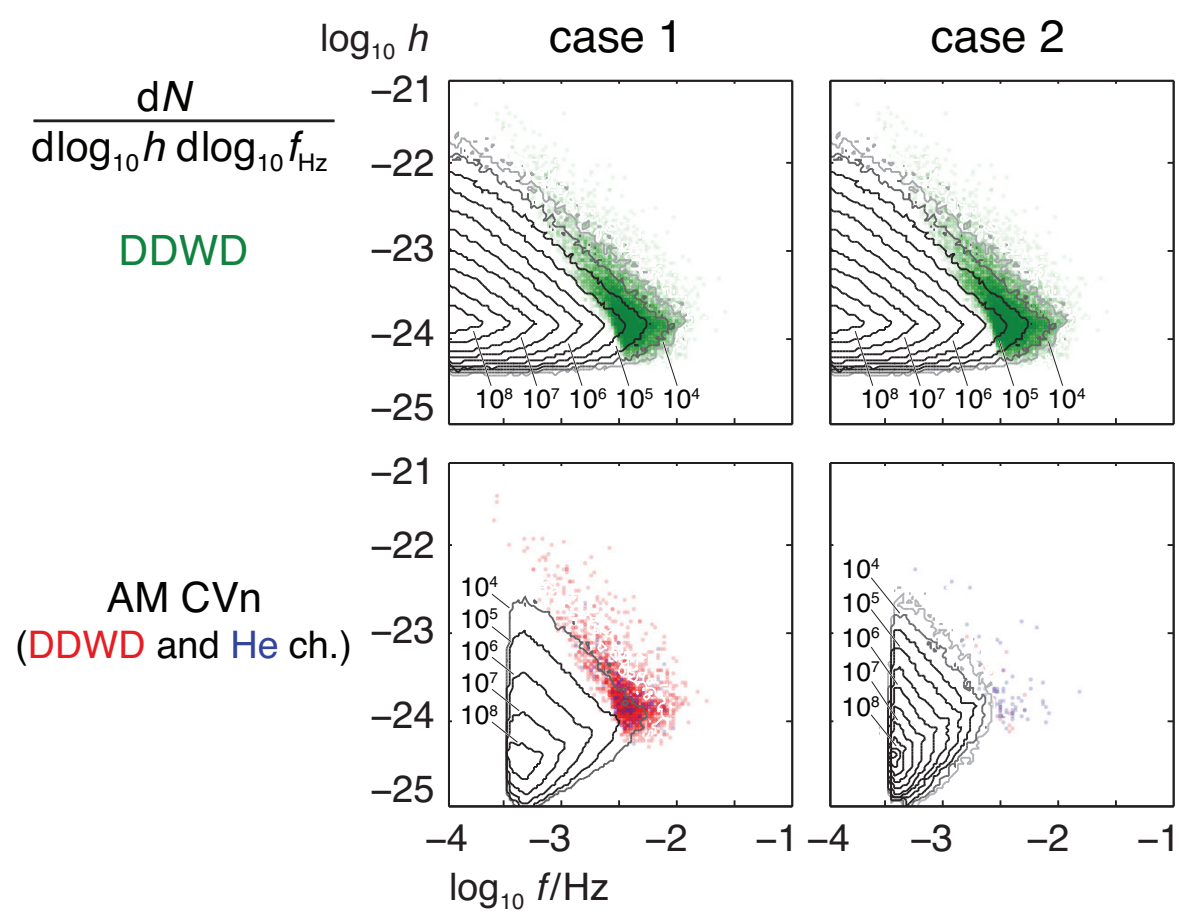

Figure 36: The number density as a function of GW-strain amplitude $h$ and frequency $f$ for close detached WD (top) and AM CVn stars (bottom) in Case 1 and Case 2. Green dots denote individually-detected WD binary systems (for one year of observation with the 5-Mkm detector); red dots - AM CVn systems from the WD binary channel and blue dots - AM CVn systems from the He star channel. Image reproduced with permission from [531], copyright by AAS. 


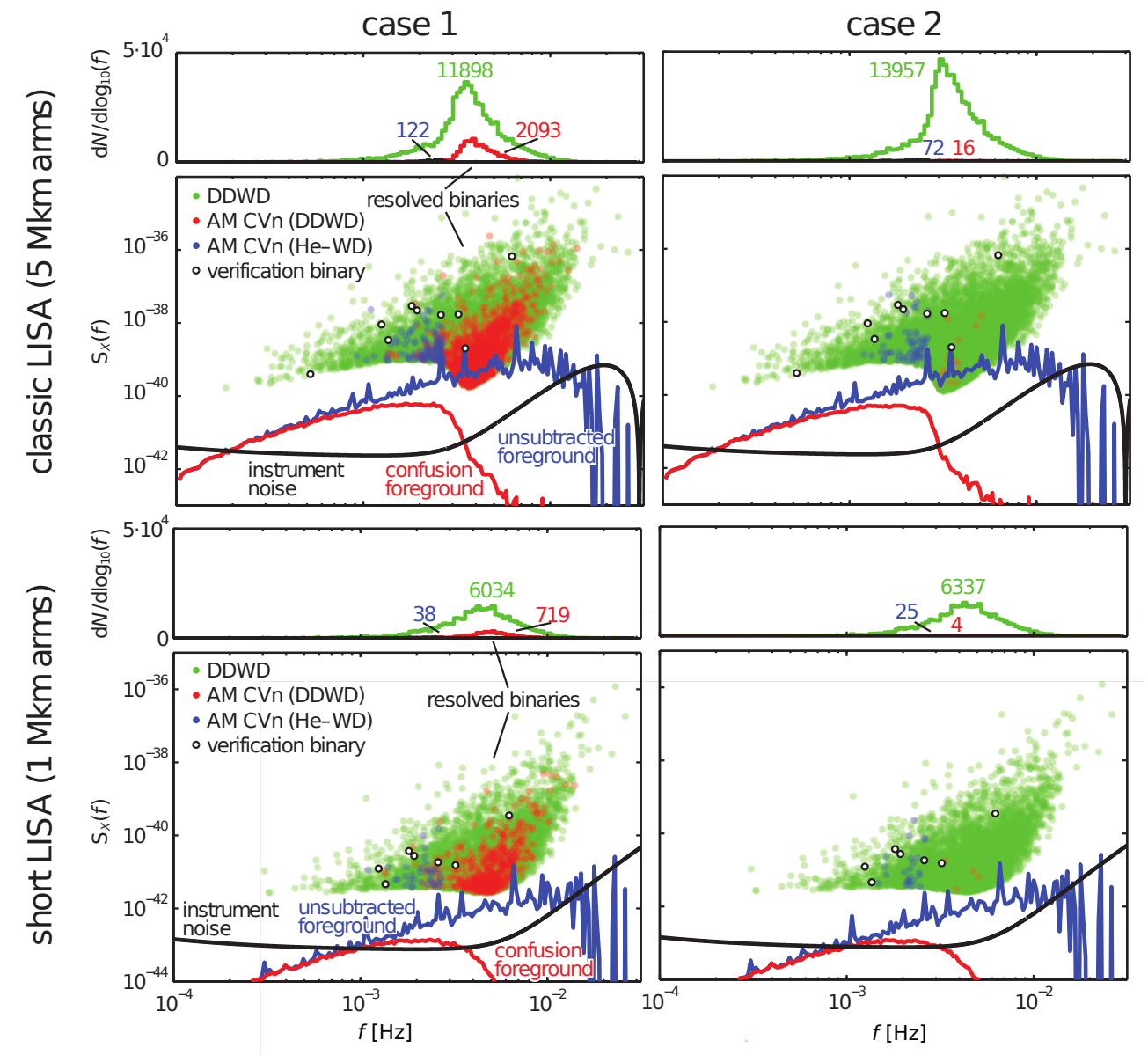

Figure 37: The frequency-space density and GW foreground of DD and AM CVn systems for two scenarios. In each subplot, the bottom panel shows the power spectral density of the unsubtracted (blue) and partially subtracted (red) foreground, compared to instrumental noise (black). The open white circles indicate the frequency and amplitude of the "verification binaries". The green dots show the individuallydetectable DD systems; the red dots show the detectable AM CVn systems formed from DD progenitors and the blue dots show the detectable AM CVn systems that arise from the He-star-WD channel. The top panel shows histograms of the detected sources in the frequency space. Image reproduced with permission from [531], copyright by AAS. 
both instrument and confusion noise and the usage of interferometric observables instead of the strain. For a $1 \mathrm{Mkm}$ detector, the effect of changing detection criterion is much more profound - by a factor of two to four. The more rigorous detection criterion changes the number of AM CVn stars more than the number of DD, since the former have smaller chirp mass and strain amplitudes. Thus, the conclusion of early studies on the dominance of detached DD in the GW foreground formation remains valid.

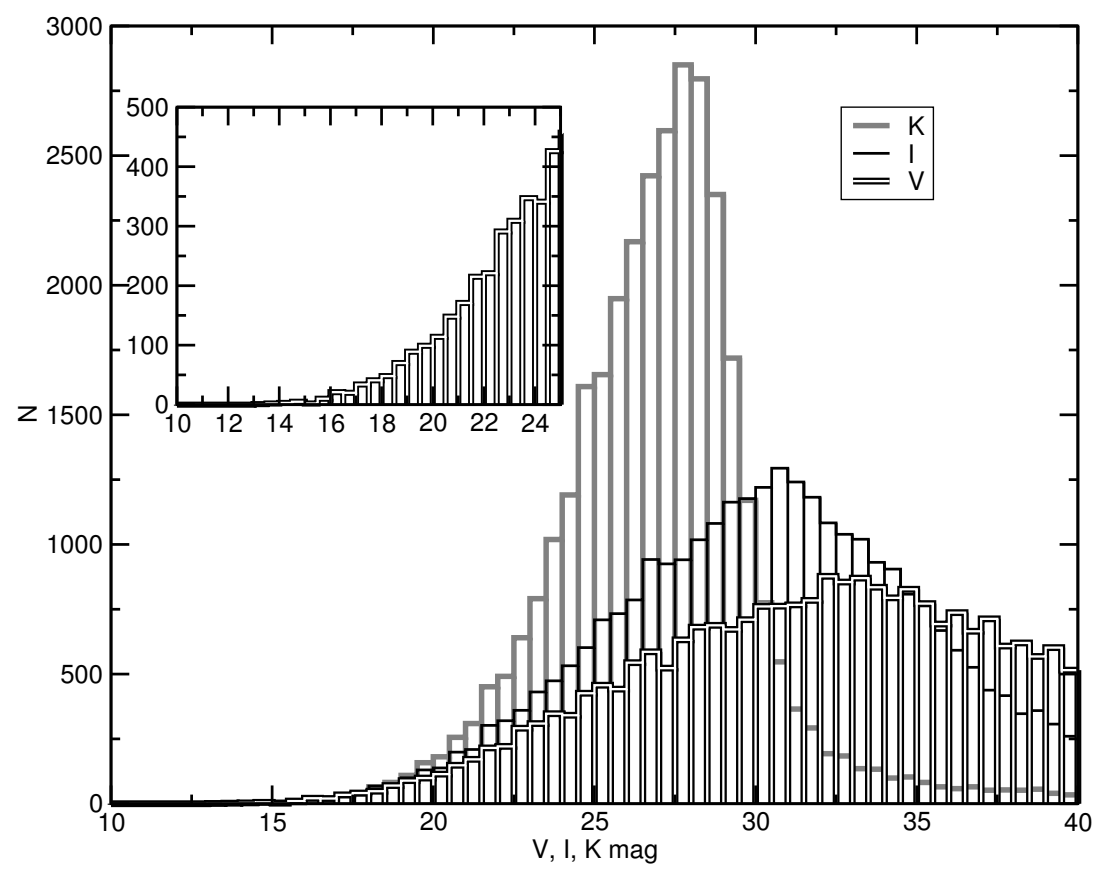

Figure 38: The histogram of apparent magnitudes of the WD binaries that are estimated to be individually detected by LISA. The black-and-white line shows the distribution in the $V$-band, the black line the distribution in the $I$-band and the grey histogram the distribution in the $K$-band. Galactic absorption is taken into account. The insert shows the bright-end tail of the $V$-band distribution. Image reproduced with permission from [509], copyright by IOP.

Peculiarly enough, as Figure 35 shows, the AM CVn-type systems appear, in fact, dominant among the "verification binaries" for LISA/eLISA: binaries that are well known from electromagnetic observations and whose radiation is estimated to be sufficiently strong to be detected; see the list of 30 promising candidates in [744] and references therein. The perspectives and strategy of discovering additional verification binaries were discussed by Littenberg et al. [433], who used as an input catalog the model from [519] with minor modifications. It was shown in [433] that for the "optimistic" scenario of the formation of AM CVn stars, $5 \times 10^{9} \mathrm{~m}$ arm-length configuration (full LISA), a $63 \%$ confidence interval of the sky-location posterior distribution area on the celestial sphere and limiting stellar magnitude of the sample $m \leq 24$ the number of candidate double-degenerate verification binaries is $\approx 560$. For the "pessimistic" scenario, short arm-length $\left(1 \times 10^{9} \mathrm{~m}\right.$, similar to eLISA), $d \Omega \leq 1 \mathrm{deg}^{2}, m \leq 20$ this number reduces to 9 . If an additional detection requirement of being eclipsing is applied, these numbers are reduced by a factor of $\sim 3$. The conclusion is that the chance for discovering new "verification binaries" is associated with the next generation of ground-based telescopes, like the 38-m European Extremely Large Telescope 
(E-ELT), ${ }^{34}$ the 30 -m US telescope (TMT), ${ }^{35}$ the James Webb Space Telescope (JWST). ${ }^{36}$ The ESA GAIA mission will also be very helpful in localization of the sources. Resolved LISA sources are expected to be most numerous in the $K$-band, and a significant fraction of them will have $K \lesssim 29$ (Figure 38), within reach of the future facilities, whilst current instruments are mostly limited by $K \lesssim(20-21)$ [509].

The most severe "astronomical" problems concerning "verification binaries" are their distances, which for most systems are only estimates, and poorly constrained component masses. Some features of "verification binaries" and prospects of detection of new objects in wide-field surveys are also discussed in [358].

The issue of "verification binaries" is related to the problem of detection of GWR sources in electromagnetic waves which is discussed in the next Section 11.

\footnotetext{
34 http://www.eso.org/public/teles-instr/e-elt/

35 http://www.tmt.org

36 http://www.jwst.nasa.gov/
} 


\section{AM CVn-Type Stars as Sources of Optical and X-Ray Emission}

Evidently, it is important to study AM CVn-stars in all possible wavebands. The accuracy of GW parameters estimation improves if the information available from electromagnetic observations is used $[519,688,687]$. GW detectors will measure a combination of parameters that determine the $\mathrm{GW}$ signal. If some of these parameters (orbital period, position on the celestial sphere) can be obtained from independent optical or X-ray observations, other parameters can be determined with higher accuracy. One of the most interesting features of the WD binaries that can be detected electromagnetically are eclipses, which have a large probability for very short $P_{\text {orb }}$ systems that can be found among candidate objects for GW detectors [117]. Eclipsing systems are especially important since they may provide information on the absolute parameters of the stars and, possibly, on variation of their orbital periods, as is already shown by the example of a 12.5-min system SDSS J065133.338+284423.37 [277]. It is estimated that GAIA will be able to detect about 200 eclipsing AM CVn stars [511].

While the total sample of AM CVn stars are optical systems, those with the shortest orbital periods have been expected to be observed with LISA, hopefully, will be observed by eLISA, and may be observed both in the optical and X-rays thanks to high mass-transfer rates (see Figure 23).

A model for electromagnetic emission properties of the ensemble of the shortest orbital period objects $\left(P_{\text {orb }} \leq 1500 \mathrm{~s}\right)$ was constructed in $[519,508,509]$. The "optimistic" model of [518] was considered.

Optical emission. The luminosity of the accretion disc around a WD can be estimated as

$$
L_{\mathrm{disc}}=\frac{1}{2} G M \dot{m}\left(\frac{1}{R}-\frac{1}{R_{\mathrm{L} 1}}\right) \operatorname{erg~s}^{-1}
$$

with $M$ and $R$ being the mass and radius of the accretor, $R_{\mathrm{L} 1}$ being the distance of the first Lagrangian point to the center of the accretor, and $\dot{m}$ being the mass transfer rate, respectively. Optical emission of the disc was modeled by a single temperature disc extending up to $70 \%$ of the Roche lobe of the accretor and radiating as a black body [817].

The emission from the donor was treated as the emission of a cooling white dwarf, using approximations to the cooling WD models of Hansen [270].

The emission from the accretor was treated as the unperturbed cooling luminosity of the white dwarf. ${ }^{37}$

The distribution of $V, I, K$-band magnitudes of the sources expected to be resolved by LISA was presented in Figure 38 [509].

X-ray emission. For the model of X-ray emission, the ROSAT $0.1-2.4 \mathrm{keV}$ X-ray band was considered, taking into account interstellar absorption. The ROSAT band was chosen because of the discovery of AM CVn itself [793] and RXJ0806.3+1527 [312] and V407 Vul [495]) as ROSAT sources and because of the possibility of comparison with the ROSAT all-sky survey.

Most AM CVn systems experience a short $\left(10^{6}-10^{7} \mathrm{yr}\right)$ "direct impact" stage in the beginning of mass-transfer [281, 518, 464]. Hence, in modeling the X-ray emission from AM CVn systems one has to distinguish two cases: the direct impact and disc accretion.

\footnotetext{
37 This is true for long-period systems, but becomes an oversimplification at shorter periods and for $P_{\text {orb }} \lesssim 40$ min, heating of the WD by accretion has to be taken into account [49].
} 
In the case of direct impact a small area of the accretor's surface is heated. One may assume that the total accretion luminosity is emitted as black body radiation with a temperature given by

$$
\left(\frac{T_{\mathrm{BB}}}{T_{\odot}}\right)^{4}=\frac{1}{s} R^{-2} L_{\mathrm{acc}}
$$

where $L_{\text {acc }}$ and $R$ are in solar units and $L_{\text {acc }}$ is defined by Eq. (75). The fraction $s$ of the surface that is radiating depends on the details of the accretion. It was set to 0.001 , consistent with expectations for a ballistic stream [448] and the observed X-ray emission of V407 Vul, the known direct-impact system [465].

In the presence of a disc, the X-ray emission was assumed to be coming from a boundary layer with temperature [616]

$$
T_{\mathrm{BL}}=5 \times 10^{5}\left(\frac{\dot{m}}{10^{18} \mathrm{~g} \mathrm{~s}^{-1}}\right)^{\frac{2}{9}}\left(\frac{M}{M_{\odot}}\right)^{\frac{1}{3}}\left(\frac{R}{5 \times 10^{8} \mathrm{~cm}}\right)^{-\frac{7}{9}} \mathrm{~K} .
$$

The systems with X-ray flux in the ROSAT band higher than $10^{-13} \mathrm{erg} \mathrm{s}^{-1} \mathrm{~cm}^{-2}$ were selected. Then, the intrinsic flux in this band, the distance and the estimate of the Galactic hydrogen absorption [494] can be used to estimate the detectable flux.

Figure 39 presents the resulting model for the sample limited by $V=20$ mag., which is typical for optically-detected AM CVn stars. In the top panel shown are 220 systems only detectable in $\mathrm{X}$-ray and 330 systems also detectable in the $V$-band. One may distinguish two subpopulations in the top panel: In the shortest period range there are systems with white-dwarf donors with such high $\dot{M}$ that even sources close to the Galactic center are detectable. Spatially, these objects are concentrated in a small area on the sky. At longer periods the X-rays get weaker (and softer) so only the systems close to the Earth can be detected. They are more evenly distributed over the sky. Several of these systems are also detectable in the optical (filled symbols). There are 30 systems that are close enough to the Earth that the donor stars can be seen as well as the discs (filled squares). Above $P=600 \mathrm{~s}$, the systems with helium-star donors show up and have a high enough mass transfer rate to be X-ray sources, the closer ones of which are also visible in the optical, as these systems always have a disc. The bottom panel shows the 1230 "conventional" AM CVn systems, detectable only by optical emission, which for most systems emanates only from their accretion disc. Of this population 170 objects closest to the Earth also have a visible donor. The majority of the optically-detectable systems with orbital periods between 1000 and $1500 \mathrm{~s}$ are expected to show outbursts due to viscous-thermal disc instability [775, 623, 379], which could enhance the chance of their discovery.

In [519] the estimates of optical and X-ray emission from AM CVn stars were applied to predict their numbers that can be detected in gravitational waves and the electromagnetic spectrum by LISA. These results were also discussed in the previous version of this review. However, in view of the cancellation of the LISA mission and the different sensitivity of eLISA, new insights into the problem of the resolution of low-frequency GW sources and the recognition that the most promising for detection of new objects with future facilities is the IR-band, these results may be considered as outdated and a new study of the problem is necessary.

To conclude this section, we stress several points that are important for the understanding of the formation and evolution of compact WD binaries.

- The major issue concerning compact WD binaries - DDs, IDDs, UCXBs - is their number. Theoretical predictions strongly depend on assumed parameters and range within an order of magnitude (see references in Section 10). The treatment of common envelopes and the distribution of stars over $q$ are, perhaps, the most crucial points. On the other hand, observational estimates suffer from numerous selection effects and resulting incompleteness of the 


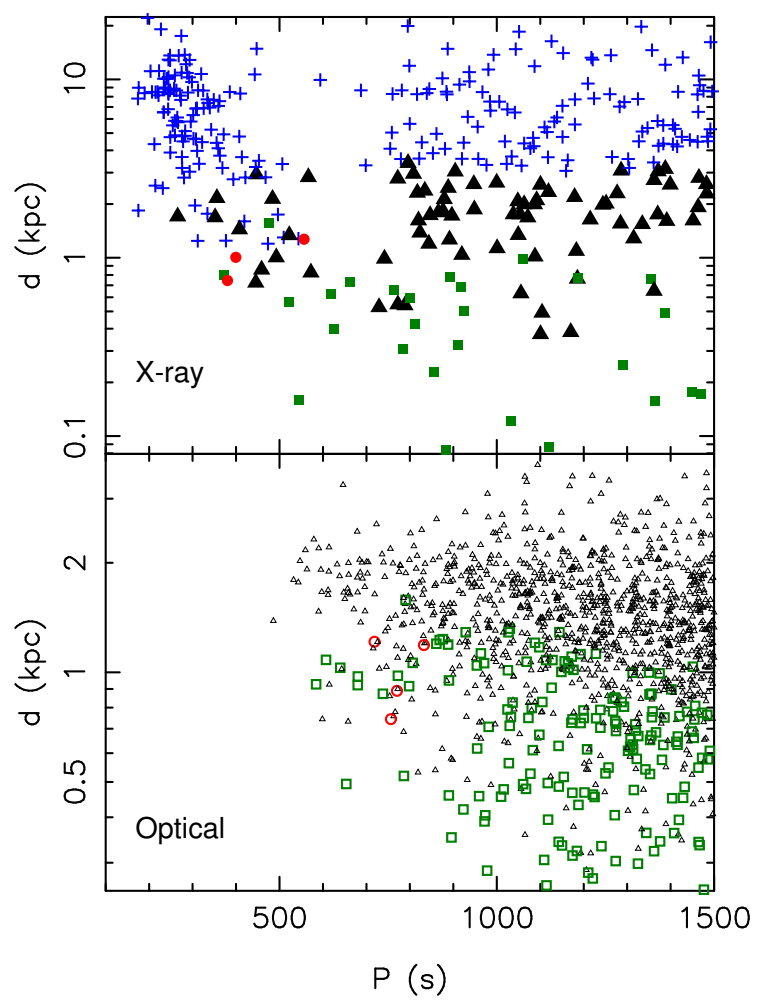

Figure 39: Distribution of short period AM CVn-type systems detectable in soft X-ray and as optical sources as a function of the orbital period and distance. Top panel: systems detectable in X-ray only (blue pluses), direct impact systems observable in X-ray and $V$-band (red filled circles), systems detectable in Xray with an optically visible donor (green squares), and systems detectable in X-ray and with an optically visible disc (large filled triangles). Bottom panel: direct impact systems (red open circles), systems with a visible donor (green squares), and systems with a visible accretion disc (small open triangles). The sample is limited by $V=20$. Image reproduced with permission from Figure 1 of [519], copyright by the authors. 
samples. For instance, the local sample of all WD is considered to be $100 \%$ complete within $13 \mathrm{pc}$ and only $85 \%$ complete within $20 \mathrm{pc}[715,716]$.

- The space density of the observed AM CVn-stars is by a factor close to 50 lower than the "optimistic" predictions, but the situation can be improved: about half of the known and candidate systems were found within the past decade, predominantly thanks to systematic searches for candidates in the SDSS and PTF data. Hopes for future discoveries may be associated with facilities that will become operative in the next 10 years, like JWST or E-ELT.

- Another key issue in the study of detached and interacting WD binaries is the determination of their distances. In this aspect, the GAIA space probe, which is aimed at constructing a three-dimensional map of our Galaxy via measurements of positions and radial velocities of about one billion stars (see [220]), appears to be the most helpful.

A failure to discover a significant number of detached and interacting double degenerates or to confirm the current ideas on their structure and evolution would mean that serious drawbacks exist either in the implementation of the known stellar evolution physics and observational statistical data in the population synthesis codes, or in our understanding of the processes occurring in compact binaries, or in the treatment of selection effects. Special attention in theoretical studies has to be paid to the onset of mass-transfer.

Above we presented some of the current ideas on the formation and evolution of compact binaries that can be interesting for general relativity and cosmology and on signals that can be expected from them in the waveband of space-born GW detectors. There is another side of the problem - the analysis of the GW signal, would it be detected. This topic is out of the scope of this brief review. We refer the reader to several papers discussing methods of detecting and subtracting individual binary signals from a data stream with many overlapping signals [118], of inferring properties of the distribution of white-dwarf binaries [170], of determining the accuracy of parameter estimation of low-mass binaries [750,653], and the discussion of the wealth of information that may be provided by eLISA [11]. 


\section{Conclusions}

The current understanding of the evolution of close binaries is firmly based on observations of many types of binary systems, from wide non-interacting pairs to very close compact binaries consisting of stellar remnants - white dwarfs, neutrons stars, and black holes. The largest uncertainties in the specific parameters of the compact binary formed at the end of the evolution of a massive binary system are related to the physical properties of the pre-supernovae: masses, magnetic fields, equations of state (for NSs), spins, possible kick velocities, etc. This situation is due to our limited understanding of both the late stages of stellar evolution and especially of the supernovae explosion mechanisms and physics of NS/BH formation.

The understanding of the origin and evolution of compact white dwarf binaries also suffers from incompleteness of our knowledge of white dwarf formation and, in particular, of the common envelope treatment. The progress in these fields, both observational and theoretical, will have a major effect on the understanding of the formation and evolution of compact binary systems. On the other hand, the phenomenological approach used to describe these uncertainties proves to be successful in explaining many observed properties of double stars of different types, so the constraints derived from the studies of binaries should be taken into account in modeling stellar evolution and supernovae explosions.

Of course, specifying and checking the initial distributions of orbital parameters of binaries and parameters of binary evolution (such as evolution in the common envelopes), as well as the modeling of accretion and merger processes stay in the short-list of important work to be done. Here an essential role belongs to detailed numerical simulations.

Further observations of compact binaries. Clearly, discoveries of new types of compact binary systems have provided the largest impetus for studies of stellar binary evolution. Wellknown examples include the discovery of X-ray binaries, relativistic binary pulsars, millisecond recycled pulsars and accreting millisecond X-ray pulsars, and close WD binaries. In the near future we expect the discovery of the $\mathrm{NS}+\mathrm{BH}$ binaries that are predicted by the massive binary evolution scenario in the form of radio pulsar binaries with BH companions [431, 424, 581]. Their immediate possible progenitors are observed as well-known Galactic system Cyg X-3 and extraGalactic objects like IC10 X-1, NGC300 X-1 harboring Wolf-Rayet stars and NS or BH.

It is very likely that we have already observed the coalescence of NS/BH binary systems as short gamma-ray bursts in other galaxies [226, 500], and the recent discovery of the IR afterglow after short/hard GRB 130603B [43, 751] provided a beautiful confirmation of the expected possible electromagnetic phenomenon ("kilonova" or "macronova") - the radioactively-powered transient, predicted by Li \& Paczyński in 1998 [416].

It is very likely that $\mathrm{NS}+\mathrm{BH}$ or $\mathrm{BH}+\mathrm{BH}$ binaries will be found first in $\mathrm{GW}$ data analysis [789, $252,157]$. The efforts of the LIGO collaboration to put constraints on compact binary coalescences from the analysis of existing GW observations are very important [3], as well as the hard work on modeling expected signal waveforms [56].

The formation and evolution of compact binaries is a very interdisciplinary field of modern astrophysics, ranging from studies of the equation of state for super-dense matter inside neutron stars and testing effects of strong gravity in relativistic compact binaries to hydrodynamical simulations of stellar winds, the formation and evolution of common envelopes, and stellar explosions. Therefore, further progress in this rapidly flourishing field of 'multi-messenger astronomy', which will be made by means of traditional astronomical observations and new tools, like gravitational wave and neutrino detectors [13], will undoubtedly have a strong impact on astronomy and astrophysics as a whole. 


\section{Acknowledgments}

The authors acknowledge the referees for careful reading of the manuscript and useful comments. The authors would like to thank L.P. Grishchuk, V.M. Lipunov, M.E. Prokhorov, A.G. Kuranov, N. Chugai, A. Tutukov, A. Fedorova, E. Ergma, M. Livio, S.F. Portegies Zwart, J.-P. Lasota, G. Dubus, A. Renzini, R. Napiwotzki, E.P.J. van den Heuvel, G. Tovmassian, J.-E. Solheim, L. van Haaften, L. Piersanti, and A. Tornambé for numerous useful discussions and joint research in the evolution of binary stars. Writing of this review would have been impossible without the longterm research cooperation of G. Nelemans. We acknowledge him also for useful discussions, help in collecting information, and updating figures. Useful discussions with participants of meetings on stellar evolution, binary stars and SN Ia are acknowledged. An intensive use of the Smithsonian/NASA ADS Astronomy Abstract Service and arXiv is acknowledged. We thank many of our colleagues for sending us (p)reprints of their publications. Y. Pachomov is acknowledged for his help in the preparation of the manuscript.

KAP was partially supported by RFBR grants 12-02-00186, 13-02-92119 and 14-02-00657. LRY was partially supported by the Presidium of the Russian Academy of Sciences program P-21 and RFBR grant 14-02-00604. 


\section{References}

[1] Aasi, J. et al. (LIGO Scientific Collaboration and Virgo Collaboration), "Prospects for Localization of Gravitational Wave Transients by the Advanced LIGO and Advanced Virgo Observatories", arXiv, e-print, (2013). [ADS], [arXiv:1304.0670 [gr-qc]]. (Cited on pages 55 and 56.)

[2] Abadie, J. et al. (LIGO Scientific Collaboration and Virgo Collaboration), "Predictions for the rates of compact binary coalescences observable by ground-based gravitational-wave detectors", Class. Quantum Grav., 27, 173001 (2010). [DOI], [ADS], [arXiv:1003.2480 [astro-ph.HE]]. (Cited on pages $17,53,54$, and 55.)

[3] Abadie, J. et al. (LIGO Scientific Collaboration and Virgo Collaboration), "Search for gravitational waves from low mass compact binary coalescence in LIGO's sixth science run and Virgo's science runs 2 and 3", Phys. Rev. D, 85, 082002 (2012). [DOI], [ADS], [arXiv:1111.7314 [gr-qc]]. (Cited on pages 17 and 109.)

[4] Abel, T., Bryan, G. L. and Norman, M. L., "The Formation of the First Star in the Universe", Science, 295, 93-98 (2002). [DOI], [ADS], [arXiv:astro-ph/0112088]. (Cited on page 8.)

[5] Abt, H. A., "Normal and abnormal binary frequencies", Annu. Rev. Astron. Astrophys., 21, 343-372 (1983). [DOI]. (Cited on page 50.)

[6] Acernese, F. et al. (VIRGO Collaboration), "The status of VIRGO", Class. Quantum Grav., 23, S63-S69 (2006). [DOI], [ADS]. (Cited on page 7.)

[7] Ahmad, A. and Jeffery, C. S., "The evolution of helium-rich subdwarf-B stars", Astrophys. Space Sci., 291, 253-260 (2004). [ADS]. (Cited on page 60.)

[8] Alexander, M. E., Chau, W. Y. and Henriksen, R. N., "Orbital evolution of a singly condensed, close binary, by mass loss from the primary and by accretion drag on the condensed member", Astrophys. J., 204, 879-888 (1976). [DOI], [ADS]. (Cited on page 36.)

[9] Althaus, L. G., Miller Bertolami, M. M. and Córsico, A. H., "New evolutionary sequences for extremely low-mass white dwarfs. Homogeneous mass and age determinations and asteroseismic prospects", Astron. Astrophys., 557, A19 (2013). [DOI], [ADS], [arXiv:1307.1882 [astro-ph.SR]]. (Cited on page 81.)

[10] Amaro-Seoane, P. et al., "Low-frequency gravitational-wave science with eLISA/NGO", Class. Quantum Grav., 29, 124016 (2012). [DOI], [ADS], [arXiv:1202.0839 [gr-qc]]. (Cited on pages 7, 93, and 94.)

[11] Amaro-Seoane, P. et al., "eLISA: Astrophysics and cosmology in the millihertz regime", GW Notes, 6, 4-110 (2013). [ADS], [arXiv:1201.3621 [astro-ph.CO]]. URL (accessed 3 March 2014): http://brownbag.lisascience.org/lisa-gw-notes/. (Cited on pages 7, 100, and 108.)

[12] Anderson, S. B., Gorham, P. W., Kulkarni, S. R., Prince, T. A. and Wolszczan, A., "Discovery of two radio pulsars in the globular cluster M15", Nature, 346, 42-44 (1990). [DOI]. (Cited on page 15.)

[13] Andersson, N. et al., "The transient gravitational-wave sky", Class. Quantum Grav., 30, 193002 (2013). [DOI], [ADS], [arXiv:1305.0816 [gr-qc]]. (Cited on pages 7 and 109.)

[14] André, P. et al., "From filamentary clouds to prestellar cores to the stellar IMF: Initial highlights from the Herschel Gould Belt Survey", Astron. Astrophys., 518, L102 (2010). [DOI], [ADS], [arXiv:1005.2618 [astro-ph.GA]]. (Cited on page 8.)

[15] Ashok, N. M., "Infrared study of the first identified helium nova V445 Puppis", Bull. Astr. Soc. India, 33, 75 (2005). [ADS]. (Cited on page 91.)

[16] Astier, P. et al., "The Supernova Legacy Survey: measurement of $\Omega_{M}, \Omega_{\Lambda}$ and $w$ from the first year data set", Astron. Astrophys., 447, 31-48 (2006). [DOI], [ADS], [arXiv:astro-ph/0510447]. (Cited on page 70. ) 
[17] Babak, S. and Grishchuk, L. P., "Energy-momentum tensor for the gravitational field", Phys. Rev. $D, \mathbf{6 1}, 024038$ (2000). (Cited on page 23.)

[18] Badenes, C., Hughes, J. P., Bravo, E. and Langer, N., "Are the Models for Type Ia Supernova Progenitors Consistent with the Properties of Supernova Remnants?", Astrophys. J., 662, 472-486 (2007). [DOI], [ADS]. (Cited on page 70.)

[19] Bagot, P., "On the progenitors of double neutron star systems", Astron. Astrophys., 322, 533-544 (1997). (Cited on page 46.)

[20] Bailes, M., "Pulsar Velocities", in van Paradijs, J., van den Heuvel, E. P. J. and Kuulkers, E., eds., Compact Stars in Binaries, Proceedings of IAU Symposium 165, 15-19 August 1994, The Hague, Netherlands, IAU Symposium, 165, pp. 213-223, (Kluwer, Dordrecht, 1996). [ADS]. (Cited on page 16.)

[21] Bailyn, C. D. and Grindlay, J. E., "Neutron stars and millisecond pulsars from accretion-induced collapse in globular clusters", Astrophys. J., 353, 159-167 (1990). [DOI], [ADS]. (Cited on page 10.)

[22] Bailyn, C. D., Jain, R. K., Coppi, P. and Orosz, J. A., "The Mass Distribution of Stellar Black Holes", Astrophys. J., 499, 367 (1998). [DOI], [ADS], [arXiv:astro-ph/9708032]. (Cited on page 18.)

[23] Barish, B. C. and Weiss, R., "LIGO and the detection of gravitational waves", Phys. Today, 52, 44-50 (1999). [DOI], [ADS]. (Cited on page 7.)

[24] Barkat, Z., Reiss, Y. and Rakavy, G., "Stars in the mass range from 7 to 10 as candidates for pulsar progenitors", Astrophys. J. Lett., 193, L21-L23 (1974). [DOI]. (Cited on page 10.)

[25] Barkov, M. V., Bisnovatyi-Kogan, G. S. and Lamzin, S. A., "The Thermal Evolution of ThorneZytkow Objects", Astron. Rep., 45, 230-235 (2001). [DOI], [ADS]. (Cited on page 45.)

[26] Barone-Nugent, R. L. et al., "Near-infrared observations of Type Ia supernovae: the best known standard candle for cosmology", Mon. Not. R. Astron. Soc., 425, 1007-1012 (2012). [DOI], [ADS], [arXiv:1204.2308 [astro-ph.CO]]. (Cited on page 7.)

[27] Baryshev, Y. V. and Paturel, G., "Statistics of the detection rates for tensor and scalar gravitational waves from the Local Galaxy universe", Astron. Astrophys., 371, 378-392 (2001). [DOI], [ADS]. (Cited on page 55.)

[28] Bate, M. R., "Stellar, brown dwarf and multiple star properties from a radiation hydrodynamical simulation of star cluster formation", Mon. Not. R. Astron. Soc., 419, 3115-3146 (2012). [DOI], [ADS], [arXiv:1110.1092 [astro-ph.SR]]. (Cited on page 12.)

[29] Bear, E. and Soker, N., "Spinning-up the envelope before entering a common envelope phase", New Astronomy, 15, 483-490 (2010). [DOI], [ADS], [arXiv:0910.0522 [astro-ph.SR]]. (Cited on page 38.)

[30] Beer, M. E., Dray, L. M., King, A. R. and Wynn, G. A., "An alternative to common envelope evolution", Mon. Not. R. Astron. Soc., 375, 1000-1008 (2007). [DOI], [ADS], [arXiv:astro-ph/0612251]. (Cited on page 40.)

[31] Behroozi, P. S., Ramirez-Ruiz, E. and Fryer, C. L., "Reinterpreting Short Gamma Ray Burst Progenitor Kicks and Time Delays Using the Host Galaxy-Dark Matter Halo Connection", arXiv, e-print, (2014). [ADS], [arXiv:1401.7986 [astro-ph.CO]]. (Cited on page 34.)

[32] Belczynski, K., Bulik, T. and Bailyn, C., "The Fate of Cyg X-1: An Empirical Lower Limit on Blackhole-Neutron-star Merger Rate", Astrophys. J. Lett., 742, L2 (2011). [DOI], [ADS], [arXiv:1107.4106 [astro-ph.GA]]. (Cited on page 19.)

[33] Belczynski, K., Bulik, T., Mandel, I., Sathyaprakash, B. S., Zdziarski, A. A. and Mikołajewska, J., "Cyg X-3: A Galactic Double Black Hole or Black-hole-Neutron-star Progenitor", Astrophys. J., 764, 96 (2013). [DOI], [ADS], [arXiv:1209.2658 [astro-ph.HE]]. (Cited on page 19.) 
[34] Belczynski, K., Kalogera, V. and Bulik, T., "A Comprehensive Study of Binary Compact Objects as Gravitational Wave Sources: Evolutionary Channels, Rates, and Physical Properties", Astrophys. J., 572, 407-431 (2002). [DOI], [ADS]. (Cited on pages 16, 35, and 46.)

[35] Belczynski, K., Kalogera, V., Rasio, F. A., Taam, R. E., Zezas, A., Bulik, T., Maccarone, T. J. and Ivanova, N., "Compact Object Modeling with the StarTrack Population Synthesis Code", Astrophys. J. Suppl. Ser., 174, 223-260 (2008). [DOI], [ADS], [astro-ph/0511811]. (Cited on page 52.)

[36] Belczynski, K. and Taam, R. E., "Galactic Populations of Ultracompact Binaries", Astrophys. J., 603, 690-696 (2004). [DOI], [ADS]. (Cited on page 78.)

[37] Belczynski, K., Wiktorowicz, G., Fryer, C. L., Holz, D. E. and Kalogera, V., "Missing Black Holes Unveil the Supernova Explosion Mechanism", Astrophys. J., 757, 91 (2012). [DOI], [ADS], [arXiv:1110.1635 [astro-ph.GA]]. (Cited on page 18.)

[38] Belkus, H., Van Bever, J. and Vanbeveren, D., "The Evolution of Very Massive Stars", Astrophys. J., 659, 1576-1581 (2007). [DOI], [ADS], [arXiv:astro-ph/0701334]. (Cited on page 8.)

[39] Benacquista, M. J. and Downing, J. M. B., "Relativistic Binaries in Globular Clusters", Living Rev. Relativity, 16, lrr-2013-4 (2013). [DOI], [ADS], [arXiv:1110.4423]. URL (accessed 4 March 2014): http://www. livingreviews.org//rr-2013-4. (Cited on pages 16 and 46.)

[40] Bender, P. L., Begelman, M. C. and Gair, J. R., "Possible LISA follow-on mission scientific objectives", Class. Quantum Grav., 30, 165017 (2013). [DOI], [ADS]. (Cited on page 7.)

[41] Benson, R. S., "Mass Exchange in Close Binaries: Accretion by the Secondary", Bull. Am. Astron. Soc., 2, 295 (1970). [ADS]. (Cited on page 36.)

[42] Benz, W., Bowers, R. L., Cameron, A. G. W. and Press, W. H., "Dynamic Mass Exchange in Doubly Degenerate Binaries. I. 0.9 and $1.2 M_{\odot}$ Stars", Astrophys. J., 348, 647-667 (1990). [DOI], [ADS]. (Cited on page 73.)

[43] Berger, E., Fong, W. and Chornock, R., "An r-process Kilonova Associated with the Short-hard GRB 130603B", Astrophys. J. Lett., 774, L23 (2013). [DOI], [ADS], [arXiv:1306.3960 [astro-ph.HE]]. (Cited on pages 56 and 109.)

[44] Bethe, H. A. and Brown, G. E., "Evolution of Binary Compact Objects That Merge", Astrophys. J., 506, 780-789 (1998). [DOI]. (Cited on pages 48 and 52.)

[45] Bhattacharya, D. and van den Heuvel, E. P. J., "Formation and evolution of binary and millisecond radio pulsars", Phys. Rep., 203, 1-124 (1991). [DOI], [ADS]. (Cited on page 21.)

[46] Bianco, F. B. et al., "Constraining Type Ia Supernovae Progenitors from Three Years of Supernova Legacy Survey Data", Astrophys. J., 741, 20 (2011). [DOI], [ADS], [arXiv:1106.4008 [astro-ph.CO]]. (Cited on page 70.)

[47] Bildsten, L., "Propagation of nuclear burning fronts on accreting neutron stars: X-ray bursts and sub-hertz noise", Astrophys. J., 438, 852-875 (1995). [DOI], [ADS]. (Cited on page 78.)

[48] Bildsten, L., Shen, K. J., Weinberg, N. N. and Nelemans, G., "Faint Thermonuclear Supernovae from AM Canum Venaticorum Binaries", Astrophys. J. Lett., 662, L95-L98 (2007). [DOI], [ADS], [arXiv:astro-ph/0703578]. (Cited on pages 83 and 91.)

[49] Bildsten, L., Townsley, D. M., Deloye, C. J. and Nelemans, G., "The Thermal State of the Accreting White Dwarf in AM Canum Venaticorum Binaries", Astrophys. J., 640, 466-473 (2006). [DOI], [ADS]. (Cited on pages 74 and 105.)

[50] Birnholtz, O. and Piran, T., "Gravitational wave memory from gamma ray bursts' jets", Phys. Rev. D, 87(12), 123007 (2013). [DOI], [ADS], [arXiv:1302.5713 [astro-ph.HE]]. (Cited on page 7.) 
[51] Bisnovatyi-Kogan, G. S., Stellar Physics. Vol. 1: Fundamental Concepts and Stellar Equilibrium, Astronomy and Astrophysics Library, (Springer, Berlin; New York, 2001). [ADS]. (Cited on page 11.)

[52] Bisnovatyi-Kogan, G. S., "Binary and recycled pulsars: 30 years after observational discovery", Phys. Usp., 176, 53-68 (2006). [DOI], [ADS]. (Cited on pages 45 and 46.)

[53] Bisnovatyi-Kogan, G. S., Stellar Physics. Vol. 2: Stellar Evolution and Stability, Astronomy and Astrophysics Library, (Springer, Berlin; New York, 2011), 2nd edition. [DOI], [ADS]. (Cited on page 11.)

[54] Bisnovatyi-Kogan, G. S. and Komberg, B. V., "Pulsars and close binary systems", Sov. Astron., 18, 217-221 (1974). (Cited on page 45.)

[55] Blaauw, A., "On the origin of the O- and B-type stars with high velocities (the 'run-away' stars), and some related problems", Bull. Astron. Inst. Neth., 15, 265-290 (1961). (Cited on page 32.)

[56] Blanchet, L., "Gravitational Radiation from Post-Newtonian Sources and Inspiralling Compact Binaries", Living Rev. Relativity, 17, lrr-2014-2 (2014). [DOI], [ADS], [arXiv:1310.1528 [gr-qc]]. URL (accessed 18 March 2014): http://www.livingreviews.org//rr-2014-2. (Cited on page 109.)

[57] Bloom, J. S. et al., "A Compact Degenerate Primary-star Progenitor of SN 2011fe", Astrophys. J. Lett., 744, L17 (2012). [DOI], [ADS], [arXiv:1111.0966 [astro-ph.HE]]. (Cited on pages 64 and 65.)

[58] Blundell, K. M., Bowler, M. G. and Schmidtobreick, L., "SS 433: Observation of the Circumbinary Disk and Extraction of the System Mass", Astrophys. J. Lett., 678, L47-L50 (2008). [DOI], [ADS]. (Cited on page 20.)

[59] Boersma, J., "Mathematical theory of the two-body problem with one of the masses decreasing with time", Bull. Astron. Inst. Neth., 15, 291-301 (1961). [ADS]. (Cited on page 32.)

[60] Boffi, F. R. and Branch, D., "Radio Emission from Type Ia Supernovae as a Test of Symbiotic Star Progenitor Systems", Publ. Astron. Soc. Pac., 107, 347 (1995). [DOI], [ADS]. (Cited on page 69.)

[61] Bogomazov, A. I., Lipunov, V. M. and Tutukov, A. V., "Evolution of close binaries and gammaray bursts", Astron. Rep., 51, 308-317 (2007). [DOI], [ADS], [arXiv:astro-ph/0607329]. (Cited on page 46.)

[62] Bondi, H., "On spherically symmetrical accretion", Mon. Not. R. Astron. Soc., 112, 195 (1952). [ADS]. (Cited on page 67.)

[63] Botticella, M. T., Smartt, S. J., Kennicutt, R. C., Cappellaro, E., Sereno, M. and Lee, J. C., "A comparison between star formation rate diagnostics and rate of core collapse supernovae within 11 Mpc", Astron. Astrophys., 537, A132 (2012). [DOI], [ADS], [arXiv:1111.1692 [astro-ph.CO]]. (Cited on page 17.)

[64] Bours, M. C. P., Toonen, S. and Nelemans, G., "Single degenerate supernova type Ia progenitors. Studying the influence of different mass retention efficiencies", Astron. Astrophys., 552, A24 (2013). [DOI], [arXiv:1302.2629 [astro-ph.SR]]. (Cited on pages 68 and 69.)

[65] Bours, M. C. P. et al., "Precise parameters for both white dwarfs in the eclipsing binary CSS 41177", Mon. Not. R. Astron. Soc., 438, 3399-3408 (2014). [DOI], [ADS], [arXiv:1401.1503 [astro-ph.SR]]. (Cited on page 81.)

[66] Bragaglia, A., Greggio, L., Renzini, A. and D’Odorico, S., "Double Degenerates among DA white dwarfs", Astrophys. J., 365, L13-L17 (1990). [DOI]. (Cited on page 79.)

[67] Branch, D., Livio, M., Yungelson, L. R., Boffi, F. R. and Baron, E., "In Search of the Progenitors of Type Ia Supernovae", Publ. Astron. Soc. Pac., 107, 1019-1029 (1995). [DOI], [ADS]. (Cited on page 77.) 
[68] Brandt, N. and Podsiadlowski, P., "The effects of high-velocity supernova kicks on the orbital properties and sky distributions of neutron-star binaries", Mon. Not. R. Astron. Soc., 274, 461-484 (1995). (Cited on page 36.)

[69] Brott, I. et al., "Rotating massive main-sequence stars. I. Grids of evolutionary models and isochrones", Astron. Astrophys., 530, A115 (2011). [DOI], [ADS], [arXiv:1102.0530 [astro-ph.SR]]. (Cited on page 11.)

[70] Brown, G. E., Heger, A., Langer, N., Lee, C.-H., Wellstein, S. and Bethe, H. A., "Formation of high mass X-ray black hole binaries", New Astronomy, 6, 457-470 (2001). [DOI], [ADS], [astroph/0102379]. (Cited on page 48.)

[71] Brown, G. E., Lee, C.-H., Wijers, R. A. M. J. and Bethe, H. A., "Evolution of black holes in the Galaxy", Phys. Rep., 333, 471-504 (2000). [DOI], [ADS]. (Cited on page 45.)

[72] Brown, W. R., Kilic, M., Allende Prieto, C., Gianninas, A. and Kenyon, S. J., "The ELM Survey. V. Merging Massive White Dwarf Binaries", Astrophys. J., 769, 66 (2013). [DOI], [ADS], [arXiv:1304.4248 [astro-ph.SR]]. (Cited on page 81.)

[73] Brown, W. R., Kilic, M., Hermes, J. J., Allende Prieto, C., Kenyon, S. J. and Winget, D. E., "A 12 Minute Orbital Period Detached White Dwarf Eclipsing Binary", Astrophys. J. Lett., 737, L23 (2011). [DOI], [ADS], [arXiv:1107.2389 [astro-ph.GA]]. (Cited on pages 57 and 81.)

[74] Brumberg, V. A., Zel'dovich, Y. B., Novikov, I. D. and Shakura, N. I., "Component masses and inclination of binary systems containing a pulsar, determined from relativistic effects", Sov. Astron. Lett., 1, 2-4 (1975). (Cited on page 14.)

[75] Bulik, T., Belczynski, K. and Prestwich, A., "IC10 X-1/NGC300 X-1: The Very Immediate Progenitors of BH-BH Binaries", Astrophys. J., 730, 140 (2011). [DOI], [ADS], [arXiv:0803.3516]. (Cited on page 19.)

[76] Büning, A. and Ritter, H., "Numerical stability of mass transfer driven by Roche lobe overflow in close binaries", Astron. Astrophys., 445, 647-652 (2006). [DOI], [ADS]. (Cited on page 41.)

[77] Burgay, M. et al., "An increased estimate of the merger rate of double neutron stars from observations of a highly relativistic system", Nature, 426, 531-533 (2003). [DOI], [ADS], [arXiv:astro-ph/0312071 [astro-ph]]. (Cited on pages 15, 16, and 53.)

[78] Burgay, M. et al., "Long-Term Variations in the Pulse Emission from PSR J0737-3039B", Astrophys. J. Lett., 624, L113-L116 (2005). [DOI], [ADS]. (Cited on page 34.)

[79] Burrows, A., "Colloquium: Perspectives on core-collapse supernova theory", Rev. Mod. Phys., 85, 245-261 (2013). [DOI], [ADS], [arXiv:1210.4921 [astro-ph.SR]]. (Cited on page 12.)

[80] Burrows, A. and Hayes, J., "Pulsar Recoil and Gravitational Radiation Due to Asymmetrical Stellar Collapse and Explosion", Phys. Rev. Lett., 76, 352-355 (1996). [DOI], [ADS], [arXiv:astroph/9511106]. (Cited on page 35.)

[81] Burrows, A., Hubbard, W. B., Saumon, D. and Lunine, J. I., "An expanded set of brown dwarf and very low mass star models", Astrophys. J., 406, 158-171 (1993). [DOI], [ADS]. (Cited on page 8.)

[82] Camilo, F. and Rasio, F. A., "Pulsars in Globular Clusters", in Rasio, F. A. and Stairs, I. H., eds., Binary Radio Pulsars, Proceedings of a meeting held at the Aspen Center for Physics, Colorado, USA, 12 - 16 January 2004, ASP Conference Series, 328, pp. 147-169, (Astronomical Society of the Pacific, San Francisco, 2005). [astro-ph/0501226]. (Cited on page 16.)

[83] Canal, R., Isern, J. and Labay, J., "Neutron star formation by collapse of white dwarfs", Space Sci. Rev., 27, 595-600 (1980). [DOI], [ADS]. (Cited on page 61.) 
[84] Canal, R., Ruiz-Lapuente, P. and Burkert, A., "The Single-Degenerate Scenario for Type Ia Supernovae in Cosmic Perspective", Astrophys. J. Lett., 456, L101 (1996). [DOI], [ADS], [arXiv:astroph/9511138]. (Cited on page 72.)

[85] Canal, R. and Schatzman, E., "Non explosive collapse of white dwarfs", Astron. Astrophys., 46, 229-235 (1976). [ADS]. (Cited on page 9.)

[86] Cappellaro, E., "The rate of supernovae", Mem. Soc. Astron. Ital., 72, 863-866 (2001). [ADS]. (Cited on page 50.)

[87] Cappellaro, E. and Turatto, M., "Supernova Types and Rates", in Vanbeveren, D., ed., The Influence of Binaries on Stellar Population Studies, Conference in Brussels, Belgium, 21-25 August 2000, Astrophysics and Space Science Library, 264, p. 199, (Kluwer Academic Publishers, Dordrecht; Boston, 2001). [ADS]. (Cited on page 69.)

[88] Carter, P. J., Steeghs, D., Marsh, T. R., Kupfer, T., Copperwheat, C. M., Groot, P. J. and Nelemans, G., "The AM Canum Venaticorum binary SDSS J173047.59+554518.5", Mon. Not. R. Astron. Soc., 437, 2894-2900 (2014). [DOI], [ADS], [arXiv:1311.0008 [astro-ph.SR]]. (Cited on page 89.)

[89] Carter, P. J. et al., "The helium-rich cataclysmic variable SBSS 1108+574", Mon. Not. R. Astron. Soc., 431, 372-382 (2013). [DOI], [ADS], [arXiv:1301.6761 [astro-ph.SR]]. (Cited on page 79.)

[90] Carter, P. J. et al., "A search for the hidden population of AM CVn binaries in the Sloan Digital Sky Survey", Mon. Not. R. Astron. Soc., 429, 2143-2160 (2013). [DOI], [ADS], [arXiv:1211.6439 [astro-ph.SR]]. (Cited on pages 79 and 88.)

[91] Carter, P. J. et al., "Two new AM Canum Venaticorum binaries from the Sloan Digital Sky Survey III", Mon. Not. R. Astron. Soc., 439, 2848-2853 (2014). [DOI], [ADS], [arXiv:1312.3335 [astroph.SR]]. (Cited on page 89.)

[92] Cartwright, T. F., Engel, M. C., Heinke, C. O., Sivakoff, G. R., Berger, J. J., Gladstone, J. C. and Ivanova, N., "Galactic Ultracompact X-Ray Binaries: Empirical Luminosities", Astrophys. J., 768, 183 (2013). [DOI]. (Cited on page 78.)

[93] Casares, J., Negueruela, I., Ribó, M., Ribas, I., Paredes, J. M., Herrero, A. and Simón-Díaz, S., "A Be-type star with a black-hole companion", Nature, 505, 378-381 (2014). [DOI], [ADS], [arXiv:1401.3711 [astro-ph.SR]]. (Cited on pages 18 and 19.)

[94] Cassisi, S., Iben Jr, I. and Tornambe, A., "Hydrogen-accreting Carbon-Oxygen White Dwarfs", Astrophys. J., 496, 376 (1998). [DOI]. (Cited on page 66.)

[95] Chamel, N., Haensel, P., Zdunik, J. L. and Fantina, A. F., "On the Maximum Mass of Neutron Stars", Int. J. Mod. Phys. E, 22, 1330018 (2013). [DOI], [ADS], [arXiv:1307.3995 [astro-ph.HE]]. (Cited on page 78.)

[96] Champion, D. J., Lorimer, D. R., McLaughlin, M. A., Cordes, J. M., Arzoumanian, Z., Weisberg, J. M. and Taylor, J. H., "PSR J1829+2456: a relativistic binary pulsar", Mon. Not. R. Astron. Soc., 350, L61-L65 (2004). [DOI], [ADS], [arXiv:astro-ph/0403553]. (Cited on page 15.)

[97] Chen, H.-L., Woods, T. E., Yungelson, L. R., Gilfanov, M. and Han, Z., "Next generation population synthesis of accreting white dwarfs: I. Hybrid calculations using BSE + MESA", arXiv, e-print, (2014). [ADS], [arXiv:1404.4450 [astro-ph.SR]]. (Cited on page 68.)

[98] Chen, M. C., Herwig, F., Denissenkov, P. A. and Paxton, B., "The dependence of the evolution of $\mathrm{SN}$ type Ia progenitors on the $\mathrm{C}$ burning rate uncertainty and parameters of convective boundary mixing", arXiv, e-print, (2013). [ADS], [arXiv:1310.1898 [astro-ph.SR]]. (Cited on page 9.)

[99] Cherepashchuk, A. M., "Masses of black holes in binary stellar systems", Phys. Usp., 39, 759-780 (1996). [DOI], [ADS]. (Cited on page 18.) 
[100] Cherepashchuk, A. M., "Search for black holes", Phys. Usp., 46, 335-371 (2003). [DOI], [ADS]. (Cited on page 18.)

[101] Cherepashchuk, A. M., Sunyaev, R. A., Postnov, K. A., Antokhina, E. A. and Molkov, S. V., "Peculiar nature of hard X-ray eclipse in SS433 from INTEGRAL observations", Mon. Not. $R$. Astron. Soc., 397, 479-487 (2009). [DOI], [ADS], [arXiv:0811.0069]. (Cited on pages 19 and 20.)

[102] Chevalier, R. A., "The radio and X-ray emission from type II supernovae", Astrophys. J., 259, 302-310 (1982). [DOI], [ADS]. (Cited on page 69.)

[103] Chevalier, R. A., "Self-similar solutions for the interaction of stellar ejecta with an external medium", Astrophys. J., 258, 790-797 (1982). [DOI], [ADS]. (Cited on page 69.)

[104] Chevalier, R. A., "Neutron star accretion in a stellar envelope", Astrophys. J. Lett., 411, L33-L36 (1993). [DOI], [ADS]. (Cited on page 45.)

[105] Chevalier, R. A., "Common Envelope Evolution Leading to Supernovae with Dense Interaction", Astrophys. J. Lett., 752, L2 (2012). [DOI], [ADS], [arXiv:1204.3300 [astro-ph.HE]]. (Cited on page 46.)

[106] Chini, R., Hoffmeister, V. H., Nasseri, A., Stahl, O. and Zinnecker, H., "A spectroscopic survey on the multiplicity of high-mass stars", Mon. Not. R. Astron. Soc., 424, 1925-1929 (2012). [DOI], [ADS], [arXiv:1205.5238 [astro-ph.SR]]. (Cited on page 12.)

[107] Chomiuk, L., "SN 2011fe: A Laboratory for Testing Models of Type Ia Supernovae", Publ. Astron. Soc. Australia, 30, e046 (2013). [DOI], [ADS], [arXiv:1307.2721 [astro-ph.HE]]. (Cited on page 65.)

[108] Chomiuk, L. and Povich, M. S., "Toward a Unification of Star Formation Rate Determinations in the Milky Way and Other Galaxies", Astron. J., 142, 197 (2011). [DOI], [ADS], [arXiv:1110.4105 [astro-ph.GA]]. (Cited on pages 9 and 17.)

[109] Chugai, N. N., "Pulsar Space Velocities and Neutrino Chirality", Sov. Astron. Lett., 10, 87 (1984). (Cited on page 34.)

[110] Chugai, N. N., "Circumstellar Na I and Ca II absorption lines of type Ia supernovae in the symbiotic scenario", Astron. Lett., 34, 389-396 (2008). [DOI], [ADS], [arXiv:0801.4468]. (Cited on page 70.)

[111] Chugai, N. N. and Yungelson, L. R., "Type Ia supernovae in dense circumstellar gas", Astron. Lett., 30, 65-72 (2004). [DOI], [astro-ph/0308297]. (Cited on page 77.)

[112] Claeys, J. S. W., Pols, O. R., Izzard, R. G., Vink, J. and Verbunt, F. W. M., "Theoretical uncertainties of the Type Ia supernova rate", Astron. Astrophys., 563, A83 (2014). [DOI], [ADS], [arXiv:1401.2895 [astro-ph.SR]]. (Cited on page 69.)

[113] Clark, J. P. A. and Eardley, D. M., "Evolution of close neutron star binaries", Astrophys. J., 215, 311-322 (1977). [DOI], [ADS]. (Cited on page 7.)

[114] Clark, J. P. A., van den Heuvel, E. P. J. and Sutantyo, W., "Formation of neutron star binaries and their importance for gravitational radiation", Astron. Astrophys., 72, 120-128 (1979). (Cited on page 7.)

[115] Close, L. M., Thatte, N., Nielsen, E. L., Abuter, R., Clarke, F. and Tecza, M., "New Photometry and Spectra of AB Doradus C: An Accurate Mass Determination of a Young Low-Mass Object with Theoretical Evolutionary Tracks", Astrophys. J., 665, 736-743 (2007). [DOI], [ADS], [arXiv:astroph/0703564]. (Cited on page 8.)

[116] Colgate, S. A., "Neutron-Star Formation, Thermonuclear Supernovae, and Heavy-Element Reimplosion", Astrophys. J., 163, 221-230 (1971). [DOI], [ADS]. (Cited on page 45.) 
[117] Cooray, A., Farmer, A. J. and Seto, N., "The Optical Identification of Close White Dwarf Binaries in the Laser Interferometer Space Antenna Era", Astrophys. J. Lett., 601, L47-L50 (2004). [DOI], [ADS]. (Cited on page 105.)

[118] Cornish, N. J. and Larson, S. L., "LISA data analysis: Source identification and subtraction", Phys. Rev. D, 67, 103001 (2003). [DOI], [ADS], [arXiv:astro-ph/0301548]. (Cited on page 108.)

[119] Corongiu, A., Kramer, M., Stappers, B. W., Lyne, A. G., Jessner, A., Possenti, A., D’Amico, N. and Löhmer, O., "The binary pulsar PSR J1811-1736: evidence of a low amplitude supernova kick", Astron. Astrophys., 462, 703-709 (2007). [DOI], [ADS], [astro-ph/0611436]. (Cited on page 34.)

[120] Corradi, R. L. M., "Close binary central stars of planetary nebulae and V458 Vul", Mem. Soc. Astron. Ital., 83, 811 (2012). [ADS]. (Cited on page 80.)

[121] Cox, J. P. and Giuli, R. T., Principles of Stellar Structure, (Gordon and Breach, New York, 1968). (Cited on page 11.)

[122] Crowder, J. and Cornish, N. J., "Beyond LISA: Exploring future gravitational wave missions", Phys. Rev. D, 72, 083005 (2005). [DOI], [ADS], [arXiv:gr-qc/0506015]. (Cited on page 7.)

[123] Crowther, P. A., "Physical Properties of Wolf-Rayet Stars", Annu. Rev. Astron. Astrophys., 45, 177-219 (2007). [DOI], [ADS], [arXiv:astro-ph/0610356]. (Cited on page 48.)

[124] Crowther, P. A., Barnard, R., Carpano, S., Clark, J. S., Dhillon, V. S. and Pollock, A. M. T., "NGC 300 X-1 is a Wolf-Rayet/black hole binary", Mon. Not. R. Astron. Soc., 403, L41-L45 (2010). [DOI], [ADS], [arXiv:1001.4616 [astro-ph.SR]]. (Cited on pages 19 and 48.)

[125] Crowther, P. A., Schnurr, O., Hirschi, R., Yusof, N., Parker, R. J., Goodwin, S. P. and Kassim, H. A., "The R136 star cluster hosts several stars whose individual masses greatly exceed the accepted $150 M_{\odot}$ stellar mass limit", Mon. Not. R. Astron. Soc., 408, 731-751 (2010). [DOI], [ADS], [arXiv:1007.3284 [astro-ph.SR]]. (Cited on page 8.)

[126] Cutler, C. and Thorne, K. S., "An Overview of Gravitational-Wave Sources", in Bishop, N. T. and Maharaj, S. D., eds., General Relativity and Gravitation, Proceedings of the 16th International Conference on General Relativity and Gravitation, Durban, South Africa, 15-21 July, 2001, pp. 72-111, (World Scientific, Singapore; River Edge, NJ, 2002). [gr-qc/0204090]. (Cited on page 54.)

[127] Dale, D. A. et al., "The Wyoming Survey for H $\alpha$. II. H $\alpha$ Luminosity Functions at $z \approx 0.16,0.24$, 0.32, and 0.40", Astrophys. J. Lett., 712, L189-L193 (2010). [DOI], [ADS], [arXiv:1003.0463 [astroph.CO]]. (Cited on page 17.)

[128] Dan, M., Rosswog, S., Brüggen, M. and Podsiadlowski, P., "The structure and fate of white dwarf merger remnants", Mon. Not. R. Astron. Soc., 438, 14-34 (2014). [DOI], [ADS], [arXiv:1308.1667 [astro-ph.HE]]. (Cited on pages 60,74, 75, and 76.)

[129] Dan, M., Rosswog, S., Guillochon, J. and Ramirez-Ruiz, E., "Prelude to A Double Degenerate Merger: The Onset of Mass Transfer and Its Impact on Gravitational Waves and Surface Detonations", Astrophys. J., 737, 89 (2011). [DOI], [ADS], [arXiv:1101.5132 [astro-ph.HE]]. (Cited on page 74.)

[130] Dan, M., Rosswog, S., Guillochon, J. and Ramirez-Ruiz, E., "How the merger of two white dwarfs depends on their mass ratio: orbital stability and detonations at contact", Mon. Not. R. Astron. Soc., 422, 2417-2428 (2012). [DOI], [ADS], [arXiv:1201.2406 [astro-ph.HE]]. (Cited on pages 74 and 77.)

[131] D'Antona, F., Ventura, P., Burderi, L. and Teodorescu, A., "Modeling the Closest Double Degenerate System RX J0806.3+1527 and Its Decreasing Period", Astrophys. J., 653, 1429-1434 (2006). [DOI], [ADS], [arXiv:astro-ph/0606577]. (Cited on page 88.) 
[132] Davydov, V. V., Esipov, V. F. and Cherepashchuk, A. M., "Spectroscopic monitoring of SS 433: A search for long-term variations of kinematic model parameters", Astron. Rep., 52, 487-506 (2008). [DOI], [ADS]. (Cited on page 20.)

[133] De Donder, E. and Vanbeveren, D., "The influence of binaries on galactic chemical evolution", New Astron. Rev., 48, 861-975 (2004). [DOI], [ADS], [arXiv:astro-ph/0410024]. (Cited on page 52.)

[134] De Donder, E. and Vanbeveren, D., "The influence of neutron star mergers on the galactic chemical enrichment of r-process elements", New Astronomy, 9, 1-16 (2004). [DOI], [ADS]. (Cited on page 52.)

[135] De Donder, E., Vanbeveren, D. and van Bever, J., "The number of O-type runaways, the number of O and Wolf-Rayet stars with a compact companion and the formation rate of double pulsars predicted by massive close binary evolution.", Astron. Astrophys., 318, 812-818 (1997). [ADS]. (Cited on pages 44 and 46 .)

[136] de Freitas Pacheco, J. A., Regimbau, T., Vincent, S. and Spallicci, A., "Expected coalescence rates of NS-NS binaries for laser beam interferometers", Int. J. Mod. Phys. D, 15, 235-250 (2006). [DOI], [ADS], [arXiv:astro-ph/0510727 [astro-ph]]. (Cited on page 52.)

[137] De Greve, J. P. and Vanbeveren, D., "Close binary systems before and after mass transfer: A comparison of observations and theory", Astrophys. Space Sci., 68, 433-457 (1980). [DOI], [ADS]. (Cited on page 28.)

[138] de Kool, M., "Common envelope evolution and double cores of planetary nebulae", Astrophys. J., 358, 189-195 (1990). [DOI]. (Cited on page 38.)

[139] De Marco, O., Passy, J.-C., Moe, M., Herwig, F., Mac Low, M.-M. and Paxton, B., "On the $\alpha$ formalism for the common envelope interaction", Mon. Not. R. Astron. Soc., 411, 2277-2292 (2011). [DOI], [ADS], [arXiv:1010.4374 [astro-ph.SR]]. (Cited on pages 39, 40, and 41.)

[140] De Marco, O., Sandquist, E. L., Mac Low, M.-M., Herwig, F. and Taam, R. E., "Wolf-Rayet Central Stars and the Binary Evolution Channel", in Reyes-Ruiz, M. and Vázquez-Semadeni, E., eds., Energetics of Cosmic Plasmas, The eight Mexico-Texas Conference on Astrophysics, held in Mexico City, Mexico, October 31-November 2, 2002, Rev. Mex. Astron. Astrof. (SC), 18, pp. 24-30, (UNAM, Mexico City, 2003). [ADS]. (Cited on page 39.)

[141] de Val-Borro, M., Karovska, M. and Sasselov, D., "Numerical Simulations of Wind Accretion in Symbiotic Binaries", Astrophys. J., 700, 1148-1160 (2009). [DOI], [ADS]. (Cited on page 67.)

[142] Deloye, C. J., Taam, R. E., Winisdoerffer, C. and Chabrier, G., "The thermal evolution of the donors in AM Canum Venaticorum binaries", Mon. Not. R. Astron. Soc., 381, 525-542 (2007). [DOI], [ADS]. (Cited on pages 85 and 87.)

[143] Denissenkov, P. A., Herwig, F., Bildsten, L. and Paxton, B., "MESA Models of Classical Nova Outbursts: The Multicycle Evolution and Effects of Convective Boundary Mixing", Astrophys. J., 762, 8 (2013). [DOI], [ADS], [arXiv:1210.5209 [astro-ph.SR]]. (Cited on page 60.)

[144] Dermine, T., Jorissen, A., Siess, L., Frankowski, A. and Dermine, T., "Radiation Pressure Effect on the Roche Lobe", in Prša, A. and Zejda, M., eds., Binaries - Key to Comprehension of the Universe, Proceedings of a conference held June 8-12, 2009 in Brno, Czech Republic, ASP Conference Series, 435, p. 85, (Astronomical Society of the Pacific, San Francisco, 2010). [ADS]. (Cited on page 26.)

[145] Dessart, L., Blondin, S., Hillier, D. J. and Khokhlov, A., "Constraints on the explosion mechanism and progenitors of type Ia supernovae", arXiv, e-print, (2013). [ADS], [arXiv:1310.7747 [astroph.SR]]. (Cited on page 65.)

[146] Dessart, L., Burrows, A., Ott, C. D., Livne, E., Yoon, S.-C. and Langer, N., "Multidimensional Simulations of the Accretion-induced Collapse of White Dwarfs to Neutron Stars", Astrophys. J., 644, 1063-1084 (2006). [DOI], [ADS]. (Cited on pages 10 and 34.) 
[147] Dewey, R. J. and Cordes, J. M., "Monte Carlo simulations of radio pulsars and their progenitors", Astrophys. J., 321, 780-798 (1987). [DOI]. (Cited on page 51.)

[148] Dewi, J. D. M. and Pols, O. R., "The late stages of evolution of helium star-neutron star binaries and the formation of double neutron star systems", Mon. Not. R. Astron. Soc., 344, 629-643 (2003). [DOI], [astro-ph/0306066]. (Cited on page 34.)

[149] Dewi, J. D. M. and van den Heuvel, E. P. J., "The formation of the double neutron star pulsar J0737-3039", Mon. Not. R. Astron. Soc., 349, 169-172 (2004). [DOI]. (Cited on page 46.)

[150] Dhurandhar, S. V. and Tinto, M., "Time-Delay Interferometry", Living Rev. Relativity, 8, lrr-2005-4 (2005). [DOI], [ADS]. URL (accessed 4 March 2014): http://www.livingreviews.org/lrr-2005-4. (Cited on page 99.)

[151] Di Stefano, R., "The Progenitors of Type Ia Supernovae. I. Are they Supersoft Sources?", Astrophys. J., 712, 728-733 (2010). [DOI], [ADS]. (Cited on page 72.)

[152] Di Stefano, R. and Kilic, M., "The Absence of Ex-companions in Type Ia Supernova Remnants", Astrophys. J., 759, 56 (2012). [DOI], [ADS], [arXiv:1205.3168 [astro-ph.SR]]. (Cited on page 72.)

[153] Di Stefano, R., Voss, R. and Claeys, J. S. W., "Spin-up/Spin-down Models for Type Ia Supernovae", Astrophys. J. Lett., 738, L1 (2011). [DOI], [ADS], [arXiv:1102.4342 [astro-ph.HE]]. (Cited on page 72.)

[154] Domínguez, I., Piersanti, L., Bravo, E., Tornambé, A., Straniero, O. and Gagliardi, S., "Rotating Type Ia SN Progenitors: Explosion and Light Curves", Astrophys. J., 644, 21-29 (2006). [DOI], [ADS]. (Cited on page 7.)

[155] Domínguez, I., Piersanti, L., Straniero, O. and Tornambè, A., "Is the cosmological use of SNe Ia reliable?", Mem. Soc. Astron. Ital., 78, 549 (2007). [ADS]. (Cited on page 7.)

[156] Dominguez, I., Straniero, O., Tornambe, A. and Isern, J., "On the Formation of Massive C-O White Dwarfs: The Lifting Effect of Rotation", Astrophys. J., 472, 783 (1996). [DOI], [ADS]. (Cited on page 11.)

[157] Dominik, M., Belczynski, K., Fryer, C. L., Holz, D. E., Berti, E., Bulik, T., Mandel, I. and O'Shaughnessy, R., "Double Compact Objects. I. The Significance of the Common Envelope on Merger Rates", Astrophys. J., 759, 52 (2012). [DOI], [ADS], [arXiv:1202.4901 [astro-ph.HE]]. (Cited on pages $16,46,52,55$, and 109.)

[158] Dominik, M., Belczynski, K., Fryer, C. L., Holz, D. E., Berti, E., Bulik, T., Mandel, I. and O'Shaughnessy, R., "Double Compact Objects. II. Cosmological Merger Rates", Astrophys. J., 779, 72 (2013). [DOI], [ADS], [arXiv:1308.1546 [astro-ph.HE]]. (Cited on page 55.)

[159] Dong, S., Katz, B., Kushnir, D. and Prieto, J. L., "Type Ia Supernovae with Bi-Modal Explosions Are Common - Possible Smoking Gun for Direct Collisions of White-Dwarfs", arXiv, e-print, (2014). [ADS], [arXiv:1401.3347 [astro-ph.HE]]. (Cited on page 77.)

[160] Dorofeev, O. F., Rodionov, V. N. and Ternov, I. M., "Anisotropic Neutrino Emission from BetaDecays in a Strong Magnetic Field", Sov. Astron. Lett., 11, 123 (1985). (Cited on page 34.)

[161] Downes, R. A., Webbink, R. F., Shara, M. M., Ritter, H., Kolb, U. and Duerbeck, H. W., "Catalog of Cataclysmic Variables (Downes+ 2001-2006)", web interface to database, Harvard-Smithsonian Center for Astrophysics, (2006). URL (accessed 17 March 2014): http://vizier.cfa.harvard.edu/viz-bin/VizieR?-source=V/123A. (Cited on page 79.)

[162] Downes, R. et al., "A Catalog and Atlas of Cataclysmic Variables", project homepage, Space Telescope Science Institute, (2006). URL (accessed 17 March 2014):

http://archive.stsci.edu/prepds/cvcat/. (Cited on page 79.) 
[163] Drake, A. J. et al., "Cataclysmic Variables from the Catalina Real-time Transient Survey", arXiv, e-print, (2014). [ADS], [arXiv:1404.3732 [astro-ph.SR]]. (Cited on page 79.)

[164] Drake, J. J. and Sarna, M. J., "X-Ray Evidence of the Common Envelope Phase of V471 Tauri", Astrophys. J. Lett., 594, L55-L58 (2003). [DOI]. (Cited on page 37.)

[165] Drout, M. R. et al., "The Fast and Furious Decay of the Peculiar Type Ic Supernova 2005ek", Astrophys. J., 774, 58 (2013). [DOI], [ADS], [arXiv:1306.2337 [astro-ph.HE]]. (Cited on page 91.)

[166] D’Souza, M. C. R., Motl, P. M., Tohline, J. E. and Frank, J., "Numerical Simulations of the Onset and Stability of Dynamical Mass Transfer in Binaries", Astrophys. J., 643, 381-401 (2006). [DOI], [ADS], [arXiv:astro-ph/0512137]. (Cited on page 73.)

[167] Duchêne, G. and Kraus, A., "Stellar Multiplicity", Annu. Rev. Astron. Astrophys., 51, 269-310 (2013). [DOI], [ADS], [arXiv:1303.3028 [astro-ph.SR]]. (Cited on page 12.)

[168] Duquennoy, A. and Mayor, M., "Multiplicity among solar-type stars in the solar neighbourhood. II. Distribution of the orbital elements in an unbiased sample", Astron. Astrophys., 248, 485-524 (1991). [ADS]. (Cited on page 12.)

[169] Durisen, R. H., "Viscous Effects in Rapidly Rotating Stars with Application to White-Dwarf Models. II. Numerical Results", Astrophys. J., 183, 215-232 (1973). [DOI], [ADS]. (Cited on page 69.)

[170] Edlund, J. A., Tinto, M., Królak, A. and Nelemans, G., "White-dwarf-white-dwarf galactic background in the LISA data", Phys. Rev. D, 71, 122003 (2005). [DOI], [ADS], [arXiv:gr-qc/0504112]. (Cited on pages 93, 94, 95, 97, and 108.)

[171] Eggleton, P., Evolutionary Processes in Binary and Multiple Stars, Cambridge Astrophysics Series, 40, (Cambridge University Press, Cambridge; New York, 2006). [ADS]. (Cited on pages 11, 21, and 28.)

[172] Eggleton, P. P., "Approximations to the radii of Roche lobes", Astrophys. J., 268, 368-369 (1983). [DOI]. (Cited on page 27.)

[173] Ekström, S. et al., "Grids of stellar models with rotation. I. Models from 0.8 to $120 M_{\odot}$ at solar metallicity $(Z=0.014)$ ", Astron. Astrophys., 537, A146 (2012). [DOI], [ADS], [arXiv:1110.5049 [astro-ph.SR]]. (Cited on page 17.)

[174] Endal, A. S. and Sofia, S., "The evolution of rotating stars. I - Method and exploratory calculations for a 7-solar-mass star", Astrophys. J., 210, 184-198 (1976). [DOI], [ADS]. (Cited on page 11.)

[175] Epelstain, N., Yaron, O., Kovetz, A. and Prialnik, D., "A thousand and one nova outbursts", Mon. Not. R. Astron. Soc., 374, 1449-1456 (2007). [DOI], [ADS], [arXiv:astro-ph/0611154]. (Cited on pages 60 and 67.)

[176] Ergma, E. and van den Heuvel, E. P. J., "On the initial progenitor masses of stellar mass black holes and neutron stars", Astron. Astrophys., 331, L29-L32 (1998). [ADS]. (Cited on pages 12 and 48.)

[177] Ergma, E. and Yungelson, L. R., "CYG X-3: can the compact object be a black hole?", Astron. Astrophys., 333, 151-158 (1998). (Cited on page 45.)

[178] Espinosa Lara, F. and Rieutord, M., "Self-consistent 2D models of fast-rotating early-type stars", Astron. Astrophys., 552, A35 (2013). [DOI], [ADS], [arXiv:1212.0778 [astro-ph.SR]]. (Cited on page 11.)

[179] Esposito, P., Israel, G. L., Sidoli, L., Mapelli, M., Zampieri, L. and Motta, S. E., "Discovery of a 6.4 h black hole binary in NGC 4490", Mon. Not. R. Astron. Soc., 436, 3380-3387 (2013). [DOI], [ADS], [arXiv:1309.6328 [astro-ph.HE]]. (Cited on page 19.) 
[180] Evans, C. R., Iben Jr, I. and Smarr, L. L., "Degenerate dwarf binaries as promising, detectable sources of gravitational radiation", Astrophys. J., 323, 129-139 (1987). [DOI], [ADS]. (Cited on page 92.)

[181] Faber, J. A. and Rasio, F. A., "Binary Neutron Star Mergers", Living Rev. Relativity, 15, lrr-2012-8 (2012). [DOI], [ADS], [arXiv:1204.3858 [gr-qc]]. URL (accessed 4 March 2014): http://www. livingreviews.org//rr-2012-8. (Cited on page 46.)

[182] Fabian, A. C., Pringle, J. E. and Rees, M. J., "Tidal capture formation of binary systems and X-ray sources in globular clusters", Mon. Not. R. Astron. Soc., 172, 15-18 (1975). [ADS]. (Cited on page 78.)

[183] Fabrika, S., "The jets and supercritical accretion disk in SS433", Astrophys. Space Phys. Rev., 12, 1-152 (2004). [ADS], [arXiv:astro-ph/0603390]. (Cited on page 20.)

[184] Fadeyev, Y. A. and Novikova, M. F., "Radial Pulsations of Helium Stars with Masses from 1 to $10 M_{\odot} "$, Astron. Lett., 29, 522-529 (2003). [DOI], [ADS]. (Cited on page 44.)

[185] Fadeyev, Y. A. and Novikova, M. F., "Radial Pulsations of Helium Stars with Masses from 10 to $50 M_{\odot} "$, Astron. Lett., 30, 707-714 (2004). [DOI], [ADS]. (Cited on page 44.)

[186] Falcon, R. E., Winget, D. E., Montgomery, M. H. and Williams, K. A., "A Gravitational Redshift Determination of the Mean Mass of White Dwarfs: DBA and DB Stars", Astrophys. J., 757, 116 (2012). [DOI], [ADS], [arXiv:1208.2009 [astro-ph.SR]]. (Cited on page 79.)

[187] Faller, J. E., Bender, P. L., Hall, J. L., Hils, D., Stebbins, R. T. and Vincent, M. A., "An antenna for laser gravitational-wave observations in space", Adv. Space Res., 9, 107-111 (1989). [DOI], [ADS]. (Cited on pages 7 and 93.)

[188] Farmer, A. J. and Phinney, E. S., "The gravitational wave background from cosmological compact binaries", Mon. Not. R. Astron. Soc., 346, 1197-1214 (2003). [DOI], [ADS], [arXiv:astro-ph/0304393]. (Cited on pages 93 and 95.)

[189] Farr, W. M., Sravan, N., Cantrell, A., Kreidberg, L., Bailyn, C. D., Mandel, I. and Kalogera, V., "The Mass Distribution of Stellar-mass Black Holes", Astrophys. J., 741, 103 (2011). [DOI], [ADS], [arXiv:1011.1459 [astro-ph.GA]]. (Cited on page 18.)

[190] Faucher-Giguère, C.-A. and Kaspi, V. M., "Birth and Evolution of Isolated Radio Pulsars", Astrophys. J., 643, 332-355 (2006). [DOI], [ADS], [arXiv:astro-ph/0512585]. (Cited on page 16.)

[191] Faucher-Giguère, C.-A. and Loeb, A., "Pulsar-black hole binaries in the Galactic Centre", Mon. Not. R. Astron. Soc., 415, 3951-3961 (2011). [DOI], [ADS], [arXiv:1012.0573 [astro-ph.HE]]. (Cited on page 18.)

[192] Faulkner, A. J. et al., "The Parkes Multibeam Pulsar Survey - V. Finding binary and millisecond pulsars", Mon. Not. R. Astron. Soc., 355, 147-158 (2004). [DOI]. (Cited on page 8.)

[193] Faulkner, A. J. et al., "PSR J1756-2251: A New Relativistic Double Neutron Star System", Astrophys. J. Lett., 618, L119-L122 (2005). [DOI]. (Cited on page 15.)

[194] Faulkner, J., "Ultrashort-Period Binaries, Gravitational Radiation, and Mass Transfer. I. The Standard Model, with Applications to WZ Sagittae and Z Camelopardalis", Astrophys. J., 170, L99-L104 (1971). [DOI], [ADS]. (Cited on page 85.)

[195] Faulkner, J., Flannery, B. P. and Warner, B., "Ultrashort-period binaries. II. HZ 29 (= AM CVn): A double-white-dwarf semidetached postcataclysmic nova?", Astrophys. J., 175, L79 (1972). [DOI]. (Cited on page 57.)

[196] Fedorova, A. V., Tutukov, A. V. and Yungelson, L. R., "Type Ia Supernovae in semi-detached binary systems", Astron. Lett., 30, 73-85 (2004). [DOI], [astro-ph/0309052]. (Cited on page 41.) 
[197] Fender, R. and Belloni, T., "GRS 1915+105 and the Disc-Jet Coupling in Accreting Black Hole Systems", Annu. Rev. Astron. Astrophys., 42, 317-364 (2004). [DOI], [ADS], [arXiv:astro-ph/0406483]. (Cited on page 17.)

[198] Ferdman, R. D. et al., "The Double Pulsar: Evidence for Neutron Star Formation without an Iron Core-collapse Supernova", Astrophys. J., 767, 85 (2013). [DOI], [arXiv:1302.2914]. (Cited on pages 35,42 , and 46 .)

[199] Fink, M., Röpke, F. K., Hillebrandt, W., Seitenzahl, I. R., Sim, S. A. and Kromer, M., "Doubledetonation sub-Chandrasekhar supernovae: can minimum helium shell masses detonate the core?", Astron. Astrophys., 514, A53 (2010). [DOI], [ADS]. (Cited on pages 74 and 76.)

[200] Flanagan, É.É. and Hughes, S. A., "Measuring gravitational waves from binary black hole coalescences. I. Signal to noise for inspiral, merger, and ringdown", Phys. Rev. D, 57, 4535-4565 (1998). [DOI], [ADS], [arXiv:gr-qc/9701039]. (Cited on page 54.)

[201] Flannery, B. P. and van den Heuvel, E. P. J., "On the origin of the binary pulsar PSR 1913+16", Astron. Astrophys., 39, 61-67 (1975). (Cited on page 42.)

[202] Foellmi, C. and Moffat, A. F. J., "Are Peculiar Wolf-Rayet Stars of Type WN8 Thorne-Zytkow Objects?", in Shara, M. M., ed., Stellar Collisions, Mergers and their Consequences, Proceedings of Symposium held at the American Museum of Natural History, New York City, NY, USA, May 30-June 2, 2000, ASP Conference Series, 263, pp. 123-129, (Astronomical Society of the Pacific, San Francisco, 2002). [ADS], [arXiv:astro-ph/0607217]. (Cited on page 45.)

[203] Foley, R. J. et al., "Type Iax Supernovae: A New Class of Stellar Explosion", Astrophys. J., 767, 57 (2013). [DOI], [ADS], [arXiv:1212.2209 [astro-ph.SR]]. (Cited on page 74.)

[204] Fong, W. and Berger, E., "The Locations of Short Gamma-Ray Bursts as Evidence for Compact Object Binary Progenitors", Astrophys. J., 776, 18 (2013). [DOI], [ADS], [arXiv:1307.0819 [astroph.HE]]. (Cited on pages 7 and 55.)

[205] For, B.-Q. and Green, E. M., "Monte Carlo simulations of post-common envelope sdB star plus white dwarf binaries", Baltic Astron., 15, 183-186 (2006). [ADS]. (Cited on page 60.)

[206] Foss, D., Wade, R. A. and Green, R. F., "Limits on the space density of double degenerates as type Ia supernova progenitors", Astrophys. J., 374, 281-287 (1991). [DOI], [ADS]. (Cited on page 79.)

[207] Foucart, F. et al., "Black-hole-neutron-star mergers at realistic mass ratios: Equation of state and spin orientation effects", Phys. Rev. D, 87, 084006 (2013). [DOI], [arXiv:1212.4810]. (Cited on page 49.)

[208] Fragos, T., Willems, B., Ivanova, N. and Kalogera, V., "Black Hole Formation in X-Ray Binaries: The Case of XTE J1118+480", in Di Salvo, T. et al., eds., The Multicolored Landscape of Compact Objects and Their Explosive Origins, Proceedings of conference held in Cefalu, Sicily, Italy, $11-$ 18 and 19-24 June 2006, AIP Conference Proceedings, 924, pp. 673-676, (American Institute of Physics, Melville, 2007). [DOI], [ADS], [arXiv:astro-ph/0612050]. (Cited on page 35.)

[209] Frieman, J. A. et al., "The Sloan Digital Sky Survey-II Supernova Survey: Technical Summary", Astron. J., 135, 338-347 (2008). [DOI], [ADS], [arXiv:0708.2749]. (Cited on page 70.)

[210] Fryer, C. L., "Mass Limits For Black Hole Formation", Astrophys. J., 522, 413-418 (1999). [DOI], [ADS], [arXiv:astro-ph/9902315]. (Cited on page 48.)

[211] Fryer, C. L., ed., Stellar Collapse, Proceedings of 'Core Collapse of Massive Stars', 200th AAS meeting, Albuquerque, NM, June 2002, Astrophysics and Space Science Library, 302, (Kluwer Academic Publishers, Dordrecht; Boston, 2004). (Cited on page 48.) 
[212] Fryer, C. L., "Compact object formation and the supernova explosion engine", Class. Quantum Grav., 30(24), 244002 (2013). [DOI], [ADS], [arXiv:1307.6141 [astro-ph.HE]]. (Cited on page 12.)

[213] Fryer, C. L., Belczynski, K., Wiktorowicz, G., Dominik, M., Kalogera, V. and Holz, D. E., "Compact Remnant Mass Function: Dependence on the Explosion Mechanism and Metallicity", Astrophys. J., 749, 91 (2012). [DOI], [ADS], [arXiv:1110.1726 [astro-ph.SR]]. (Cited on pages 18 and 48.)

[214] Fryer, C. L., Burrows, A. and Benz, W., "Population Syntheses for Neutron Star Systems with Intrinsic Kicks", Astrophys. J., 496, 333 (1998). [DOI], [ADS]. (Cited on page 35.)

[215] Fryer, C. L. and Kalogera, V., "Theoretical Black Hole Mass Distributions", Astrophys. J., 554, 548-560 (2001). [DOI], [ADS]. (Cited on page 48.)

[216] Fryer, C. L. and New, K. C. B., "Gravitational Waves from Gravitational Collapse", Living Rev. Relativity, 14, lrr-2011-1 (2011). [DOI], [ADS]. URL (accessed 4 March 2014): http://www.livingreviews.org/lrr-2011-1. (Cited on page 17.)

[217] Fukugita, M. and Peebles, P. J. E., "The Cosmic Energy Inventory", Astrophys. J., 616, 643-668 (2004). [DOI], [ADS], [arXiv:astro-ph/0406095]. (Cited on page 8.)

[218] Fuller, J. and Lai, D., "Tidal Novae in Compact Binary White Dwarfs", Astrophys. J. Lett., 756, L17 (2012). [DOI], [ADS], [arXiv:1206.0470 [astro-ph.SR]]. (Cited on page 94.)

[219] Fuller, J. and Lai, D., "Dynamical tides in compact white dwarf binaries: helium core white dwarfs, tidal heating and observational signatures", Mon. Not. R. Astron. Soc., 430, 274-287 (2013). [DOI], [ADS], [arXiv:1211.0624 [astro-ph.SR]]. (Cited on page 94.)

[220] "Gaia", project homepage, European Space Agency. URL (accessed 29 April 2014): http://sci.esa.int/gaia/. (Cited on page 108.)

[221] Gair, J. R., Vallisneri, M., Larson, S. L. and Baker, J. G., "Testing General Relativity with LowFrequency, Space-Based Gravitational-Wave Detectors", Living Rev. Relativity, 16, lrr-2013-7 (2013). [DOI], [ADS], [arXiv:1212.5575 [gr-qc]]. URL (accessed 4 March 2014): http://www.livingreviews.org/lrr-2013-7. (Cited on page 7.)

[222] Gänsicke, B. T., Koester, D., Girven, J., Marsh, T. R. and Steeghs, D., "Two White Dwarfs with Oxygen-Rich Atmospheres", Science, 327, 188 (2010). [DOI], [ADS], [arXiv:0911.2246 [astro-ph.SR]]. (Cited on page 64.)

[223] García-Berro, E., Lorén-Aguilar, P., Pedemonte, A. G., Isern, J., Bergeron, P., Dufour, P. and Brassard, P., "Evidence of a Merger of Binary White Dwarfs: The Case of GD 362", Astrophys. J. Lett., 661, L179-L182 (2007). [DOI], [ADS], [arXiv:0704.1258]. (Cited on page 76.)

[224] Ge, H., Hjellming, M. S., Webbink, R. F., Chen, X. and Han, Z., "Adiabatic Mass Loss in Binary Stars. I. Computational Method", Astrophys. J., 717, 724-738 (2010). [DOI], [ADS], [arXiv:1005.3099 [astro-ph.SR]]. (Cited on pages 38 and 41.)

[225] Ge, H., Webbink, R. F., Han, Z. and Chen, X., "Stellar adiabatic mass loss model and applications", Astrophys. Space Sci., 329, 243-248 (2010). [DOI], [ADS], [arXiv:1005.3105 [astro-ph.SR]]. (Cited on pages 38 and 41.)

[226] Gehrels, N. et al., "A short $\gamma$-ray burst apparently associated with an elliptical galaxy at redshift $z=0.225 "$, Nature, 437, 851-854 (2005). [DOI], [ADS]. (Cited on page 109.)

[227] Geier, S., "Hot subdwarf formation: Confronting theory with observation", in Montalbán, J., Noels, A. and Van Grootel, V., eds., European Physical Journal Web of Conferences, 40th Liège International Astrophysical Colloquium, Liège, Belgium, EPJ Web of Conferences, 43, 04001, (EDP Sciences, Les Ulis, France, 2013). [DOI], [ADS], [arXiv:1212.0418 [astro-ph.SR]]. (Cited on page 60.) 
[228] Geier, S., Heber, U., Heuser, C., Classen, L., O'Toole, S. J. and Edelmann, H., "The subdwarf B star SB 290 - A fast rotator on the extreme horizontal branch", Astron. Astrophys., 551, L4 (2013). [DOI], [ADS], [arXiv:1301.4129 [astro-ph.SR]]. (Cited on page 75.)

[229] Geier, S., Heber, U. and Napiwotzki, R., "Metal abundances of subdwarf B stars from SPY - a pattern emerges", Mem. Soc. Astron. Ital., 79, 723 (2008). [ADS], [arXiv:0804.1287]. (Cited on page 79.)

[230] Geier, S., Napiwotzki, R., Heber, U. and Nelemans, G., "Binaries discovered by the SPY survey. VI. Discovery of a low mass companion to the hot subluminous planetary nebula central star EGB 5 a recently ejected common envelope?", Astron. Astrophys., 528, L16 (2011). [DOI], [ADS]. (Cited on page 79.)

[231] Geier, S., Nesslinger, S., Heber, U., Przybilla, N., Napiwotzki, R. and Kudritzki, R.-P., "The Subdwarf B+ White Dwarf Binary KPD 1930+2752, a Supernova Type Ia Progenitor Candidate", in Napiwotzki, R. and Burleigh, M. R., eds., 15th European Workshop on White Dwarfs, Proceedings of the conference held 7-11 August, 2006 in Leicester, United Kingdom, ASP Conference Series, 372, p. 393, (Astronomical Society of the Pacific, San Francisco, 2007). [ADS], [arXiv:astro-ph/0612532]. (Cited on page 81.$)$

[232] Geier, S. et al., "Hot subdwarfs in binary systems and the nature of their unseen companions", Astrophys. Space Sci., 329, 91-99 (2010). [DOI], [ADS]. (Cited on page 60.)

[233] Geier, S. et al., "Orbital solutions of eight close sdB binaries and constraints on the nature of the unseen companions", Astron. Astrophys., 562, A95 (2014). [DOI], [ADS], [arXiv:1401.0446 [astroph.SR]]. (Cited on page 60.)

[234] Georgy, C., Ekström, S., Granada, A., Meynet, G., Mowlavi, N., Eggenberger, P. and Maeder, A., "Populations of rotating stars. I. Models from 1.7 to $15 M_{\odot}$ at $Z=0.014,0.006$, and 0.002 with $\Omega / \Omega_{\text {crit }}$ between 0 and 1", Astron. Astrophys., 553, A24 (2013). [DOI], [ADS], [arXiv:1303.2321 [astro-ph.SR]]. (Cited on page 11.)

[235] Georgy, C., Meynet, G., Walder, R., Folini, D. and Maeder, A., "The different progenitors of type Ib, Ic SNe, and of GRB", Astron. Astrophys., 502, 611-622 (2009). [DOI], [ADS], [arXiv:0906.2284 [astro-ph.SR]]. (Cited on page 48.)

[236] Georgy, C. et al., "Grids of stellar models with rotation. III. Models from 0.8 to $120 M_{\odot}$ at a metallicity $Z=0.002$ ", Astron. Astrophys., 558, A103 (2013). [DOI], [ADS], [arXiv:1308.2914 [astroph.SR]]. (Cited on page 11.)

[237] Gerosa, D., Kesden, M., Berti, E., O’Shaughnessy, R. and Sperhake, U., "Resonant-plane locking and spin alignment in stellar-mass black-hole binaries: A diagnostic of compact-binary formation", Phys. Rev. D, 87, 104028 (2013). [DOI], [ADS], [arXiv:1302.4442 [gr-qc]]. (Cited on pages 35 and 49.)

[238] Ghosh, K. K., Rappaport, S., Tennant, A. F., Swartz, D. A., Pooley, D. and Madhusudhan, N., "Discovery of a 3.6 hr Eclipsing Luminous X-Ray Binary in the Galaxy NGC 4214", Astrophys. J., 650, 872-878 (2006). [DOI], [astro-ph/0604466]. (Cited on page 31.)

[239] Giacconi, R., Murray, S., Gursky, H., Kellogg, E., Schreier, E., Matilsky, T., Koch, D. and Tananbaum, H., "The Third UHURU Catalog of X-Ray Sources", Astrophys. J. Suppl. Ser., 27, 37 (1974). [DOI], [ADS]. (Cited on page 79.)

[240] Giampieri, G. and Polnarev, A. G., "Detecting an anisotropic gravitational wave background with a space-borne interferometer", Mon. Not. R. Astron. Soc., 291, 149-161 (1997). [DOI], [ADS]. (Cited on page 93.)

[241] Gianninas, A., Hermes, J. J., Brown, W. R., Dufour, P., Barber, S. D., Kilic, M., Kenyon, S. J. and Harrold, S. T., "SDSS J074511.56+194926.5: Discovery of a Metal-Rich and Tidally Distorted Extremely Low Mass White Dwarf", Astrophys. J., 781, 104 (2014). [DOI], [ADS], [arXiv:1312.1665 [astro-ph.SR]]. (Cited on page 81.) 
[242] Gil-Pons, P., García-Berro, E., José, J., Hernanz, M. and Truran, J. W., "The frequency of occurrence of novae hosting an ONe white dwarf", Astron. Astrophys., 407, 1021-1028 (2003). [DOI]. (Cited on page 60.)

[243] Gilfanov, M., "X-ray binaries and star formation", Phys. Usp., 56, 714-722 (2013). [DOI]. (Cited on page 20.)

[244] Gilfanov, M. and Bogdán, Á., "An upper limit on the contribution of accreting white dwarfs to the type Ia supernova rate", Nature, 463, 924-925 (2010). [DOI], [ADS]. (Cited on page 72.)

[245] Girven, J. et al., "The unseen population of F- to K-type companions to hot subdwarf stars", Mon. Not. R. Astron. Soc., 425, 1013-1041 (2012). [DOI], [ADS], [arXiv:1205.6803 [astro-ph.SR]]. (Cited on page 60.)

[246] Gokhale, V., Peng, X. M. and Frank, J., "Evolution of Close White Dwarf Binaries", Astrophys. J., 655, 1010-1024 (2007). [DOI], [ADS]. (Cited on page 84.)

[247] Goranskij, V. P., Shugarov, S. Y., Zharova, A., Kroll, P. and Barsukova, E. A., "The progenitor and remnant of the helium nova V445 Puppis", Perem. Zvezdy (Var. Stars), 30(4) (2010). [ADS], [arXiv:1011.6063 [astro-ph.SR]]. URL (accessed 29 April 2014):

http://www.astronet.ru/db/varstars/msg/eid/PZ-30-004. (Cited on page 91.)

[248] Gosnell, N. M., Mathieu, R. D., Geller, A. M., Sills, A., Leigh, N. and Knigge, C., "Detection of white dwarf companions to blue stragglers in the open cluster NGC 188: direct evidence for recent mass transfer", Astrophys. J. Lett., 783, L8 (2014). [DOI], [ADS], [arXiv:1401.7670 [astro-ph.SR]]. (Cited on page 41.)

[249] Graur, O. and Maoz, D., "Discovery of 90 Type Ia supernovae among 700000 Sloan spectra: the Type Ia supernova rate versus galaxy mass and star formation rate at redshift $\sim 0.1 "$, Mon. Not. R. Astron. Soc., 430, 1746-1763 (2013). [DOI], [ADS], [arXiv:1209.0008 [astro-ph.CO]]. (Cited on page 7.$)$

[250] Graur, O. et al., "Supernovae in the Subaru Deep Field: the rate and delay-time distribution of Type Ia supernovae out to redshift 2", Mon. Not. R. Astron. Soc., 417, 916-940 (2011). [DOI], [ADS], [arXiv:1102.0005 [astro-ph.CO]]. (Cited on page 7.)

[251] Green, E. M., Liebert, J. and Saffer, R. A., "On The Origin Of Subdwarf B Stars and Related Metal-Rich Binaries", in Provencal, J. L., Shipman, H. L., MacDonald, J. and Goodchild, S., eds., 12th European Workshop on White Dwarfs, Proceedings of a conference held at the University of Delaware, Newark, Delaware, USA, 12 -16 June 2000, ASP Conference Series, 226, pp. 192-197, (Astronomical Society of the Pacific, San Francisco, 2001). [ADS]. (Cited on page 60.)

[252] Grishchuk, L. P., Lipunov, V. M., Postnov, K. A., Prokhorov, M. E. and Sathyaprakash, B. S., "Gravitational Wave Astronomy: In Anticipation of First Sources to be Detected", Phys. Usp., 44, 1-51 (2001). [astro-ph/0008481]. (Cited on pages 7, 54, 55, 93, and 109.)

[253] Grossman, D., Korobkin, O., Rosswog, S. and Piran, T., "The long-term evolution of neutron star merger remnants - II. Radioactively powered transients", Mon. Not. R. Astron. Soc., 439, 757-770 (2014). [DOI], [ADS], [arXiv:1307.2943 [astro-ph.HE]]. (Cited on page 56.)

[254] Guillemot, L. et al., "Fermi LAT pulsed detection of PSR J0737-3039A in the double pulsar system", Astrophys. J., 768, 169 (2013). [DOI], [arXiv:1303.7352]. (Cited on page 35.)

[255] Guillochon, J., Dan, M., Ramirez-Ruiz, E. and Rosswog, S., "Surface Detonations in Double Degenerate Binary Systems Triggered by Accretion Stream Instabilities", Astrophys. J. Lett., 709, L64-L69 (2010). [DOI], [ADS], [arXiv:0911.0416 [astro-ph.HE]]. (Cited on pages 73 and 74.)

[256] Gunn, J. E. and Ostriker, J. P., "On the Nature of Pulsars. III. Analysis of Observations", Astrophys. J., 160, 979-1002 (1970). [DOI]. (Cited on page 33.) 
[257] Hachisu, I., Kato, M. and Nomoto, K., "A New Model for Progenitor Systems of Type Ia Supernovae", Astrophys. J. Lett., 470, L97 (1996). [DOI], [ADS]. (Cited on page 68.)

[258] Hachisu, I., Kato, M. and Nomoto, K., "The Delay-Time Distribution of Type Ia Supernovae and the Single-Degenerate Model", Astrophys. J. Lett., 683, L127-L130 (2008). [DOI], [ADS], [arXiv:0805.2102]. (Cited on page 68.)

[259] Hachisu, I., Kato, M. and Nomoto, K., "Supersoft X-ray Phase of Single Degenerate Type Ia Supernova Progenitors in Early-type Galaxies", Astrophys. J. Lett., 724, L212-L216 (2010). [DOI], [ADS], [arXiv:1010.5860 [astro-ph.HE]]. (Cited on page 61.)

[260] Hachisu, I., Kato, M. and Nomoto, K., "Final Fates of Rotating White Dwarfs and Their Companions in the Single Degenerate Model of Type Ia Supernovae", Astrophys. J. Lett., 756, L4 (2012). [DOI], [ADS], [arXiv:1207.2261 [astro-ph.SR]]. (Cited on page 69.)

[261] Hachisu, I., Kato, M., Nomoto, K. and Umeda, H., "A New Evolutionary Path to Type Ia Supernovae: A Helium-rich Supersoft X-Ray Source Channel", Astrophys. J., 519, 314-323 (1999). [DOI], [ADS]. (Cited on page 41.)

[262] Hachisu, I., Kato, M., Saio, H. and Nomoto, K., "A Single Degenerate Progenitor Model for Type Ia Supernovae Highly Exceeding the Chandrasekhar Mass Limit", Astrophys. J., 744, 69 (2012). [DOI], [ADS], [arXiv:1106.3510 [astro-ph.SR]]. (Cited on page 68.)

[263] Hamann, W.-R., Gräfener, G. and Liermann, A., "The Galactic WN stars. Spectral analyses with line-blanketed model atmospheres versus stellar evolution models with and without rotation", Astron. Astrophys., 457, 1015-1031 (2006). [DOI], [ADS]. (Cited on page 48.)

[264] Hamuy, M. et al., "An asymptotic-giant-branch star in the progenitor system of a type Ia supernova", Nature, 424, 651-654 (2003). [DOI], [ADS]. (Cited on page 62.)

[265] Han, Z., "The formation of double degenerates and related objects", Mon. Not. R. Astron. Soc., 296, 1019-1040 (1998). [DOI], [ADS]. (Cited on page 95.)

[266] Han, Z., Podsiadlowski, P. and Eggleton, P. P., "A possible criterion for envelope ejection in asymptotic giant branch or first giant branch stars", Mon. Not. R. Astron. Soc., 270, 121-130 (1994). [ADS]. (Cited on page 9.)

[267] Han, Z., Podsiadlowski, P., Maxted, P. F. L. and Marsh, T. R., "The origin of subdwarf B stars - II", Mon. Not. R. Astron. Soc., 341, 669-691 (2003). [DOI], [ADS], [arXiv:astro-ph/0301380]. (Cited on page 60.)

[268] Han, Z., Podsiadlowski, P., Maxted, P. F. L., Marsh, T. R. and Ivanova, N., "The origin of subdwarf B stars - I. The formation channels", Mon. Not. R. Astron. Soc., 336, 449-466 (2002). [DOI]. (Cited on pages 41 and 60.)

[269] Han, Z. and Webbink, R. F., "Stability and energetics of mass transfer in double white dwarfs", Astron. Astrophys., 349, L17-L20 (1999). (Cited on page 84.)

[270] Hansen, B. M. S., "Cooling models for old white dwarfs", Astrophys. J., 520, 680-695 (1999). [DOI]. (Cited on page 105.)

[271] Harris, H. C. et al., "The Binary White Dwarf LHS 3236", Astrophys. J., 779, 21 (2013). [DOI], [ADS], [arXiv:1309.1173 [astro-ph.SR]]. (Cited on page 81.)

[272] Hayden, B. T. et al., "Single or Double Degenerate Progenitors? Searching for Shock Emission in the SDSS-II Type Ia Supernovae", Astrophys. J., 722, 1691-1698 (2010). [DOI], [ADS], [arXiv:1008.4797 [astro-ph.CO]]. (Cited on page 70.)

[273] Heber, U., "Hot Subdwarf Stars", Annu. Rev. Astron. Astrophys., 47, 211-251 (2009). [DOI], [ADS]. (Cited on page 60.) 
[274] Heger, A., Fryer, C. L., Woosley, S. E., Langer, N. and Hartmann, D. H., "How Massive Single Stars End Their Life", Astrophys. J., 591, 288-300 (2003). [DOI], [ADS]. (Cited on page 48.)

[275] Heinke, C. O., Ivanova, N., Engel, M. C., Pavlovskii, K., Sivakoff, G. R., Cartwright, T. F. and Gladstone, J. C., "Galactic Ultracompact X-Ray Binaries: Disk Stability and Evolution", Astrophys. J., 768, 184 (2013). [DOI]. (Cited on page 78.)

[276] Henze, M. et al., "Classical Novae as Supersoft X-ray Sources in the Andromeda Galaxy", in Di Stefano, R., Orio, M. and Moe, M., eds., Binary Paths to Type Ia Supernovae Explosions, Proceedings of IAU Symposium 281, July 2011, IAU Symposium, 281, pp. 105-112, (Cambridge University Press, Cambridge, 2013). [DOI], [ADS]. (Cited on page 61.)

[277] Hermes, J. J. et al., "Rapid Orbital Decay in the 12.75-minute Binary White Dwarf J0651+2844", Astrophys. J. Lett., 757, L21 (2012). [DOI], [ADS], [arXiv:1208.5051 [astro-ph.SR]]. (Cited on pages 57 and 105.)

[278] Herwig, F., "Evolution of Asymptotic Giant Branch Stars", Annu. Rev. Astron. Astrophys., 43, 435-479 (2005). [DOI], [ADS]. (Cited on page 9.)

[279] Herwig, F., "Evolution of Solar and Intermediate-Mass Stars", in Oswalt, T. D. and Barstow, M. A., eds., Planets, Stars and Stellar Systems. Volume 4: Stellar Structure and Evolution, pp. 397-445, (Springer, Berlin; New York, 2013). [DOI], [ADS]. (Cited on page 9.)

[280] Hicken, M., Garnavich, P. M., Prieto, J. L., Blondin, S., DePoy, D. L., Kirshner, R. P. and Parrent, J., "The Luminous and Carbon-rich Supernova 2006gz: A Double Degenerate Merger?", Astrophys. J. Lett., 669, L17-L20 (2007). [DOI], [ADS]. (Cited on page 73.)

[281] Hils, D. and Bender, P. L., "Gravitational Radiation from Helium Cataclysmics", Astrophys. J., 537, 334-341 (2000). [DOI], [ADS]. (Cited on pages 93 and 105.)

[282] Hils, D., Bender, P. L. and Webbink, R. F., "Gravitational radiation from the Galaxy", Astrophys. J., 360, 75-94 (1990). [DOI]. (Cited on pages 92 and 93.)

[283] Hjellming, M. S. and Webbink, R. F., "Thresholds for rapid mass transfer in binary systems. I. Polytropic models", Astrophys. J., 318, 794-808 (1987). [DOI]. (Cited on pages 37 and 41.)

[284] Hobbs, G., Lorimer, D. R., Lyne, A. G. and Kramer, M., "A statistical study of 233 pulsar proper motions", Mon. Not. R. Astron. Soc., 360, 974-992 (2005). [DOI], [ADS], [arXiv:astro-ph/0504584 [astro-ph]]. (Cited on page 33.)

[285] Hoeflich, P. and Khokhlov, A., "Explosion Models for Type Ia Supernovae: A Comparison with Observed Light Curves, Distances, H 0, and Q 0", Astrophys. J., 457, 500 (1996). [DOI], [ADS], [arXiv:astro-ph/9602025]. (Cited on page 73.)

[286] Höflich, P., Khokhlov, A., Wheeler, J. C., Phillips, M. M., Suntzeff, N. B. and Hamuy, M., "Maximum Brightness and Postmaximum Decline of Light Curves of Type Ia Supernovae: A Comparison of Theory and Observations", Astrophys. J. Lett., 472, L81-L84 (1996). [DOI], [ADS]. (Cited on page 73.)

[287] Holcomb, C., Guillochon, J., De Colle, F. and Ramirez-Ruiz, E., "Conditions for Successful Helium Detonations in Astrophysical Environments", Astrophys. J., 771, 14 (2013). [DOI], [ADS], [arXiv:1302.6235 [astro-ph.HE]]. (Cited on page 76.)

[288] Horiuchi, S., Beacom, J. F., Bothwell, M. S. and Thompson, T. A., "Effects of Stellar Rotation on Star Formation Rates and Comparison to Core-collapse Supernova Rates", Astrophys. J., 769, 113 (2013). [DOI], [ADS], [arXiv:1302.0287]. (Cited on page 17.)

[289] Hotan, A. W., Bailes, M. and Ord, S. M., "Geodetic Precession in PSR J1141-6545", Astrophys. J., 624, 906-913 (2005). [DOI]. (Cited on page 34.) 
[290] Hotokezaka, K., Kyutoku, K., Tanaka, M., Kiuchi, K., Sekiguchi, Y., Shibata, M. and Wanajo, S., "Progenitor Models of the Electromagnetic Transient Associated with the Short Gamma Ray Burst 130603B", Astrophys. J. Lett., 778, L16 (2013). [DOI], [ADS], [arXiv:1310.1623]. (Cited on page 56.)

[291] Howell, D. A. et al., "The type Ia supernova SNLS-03D3bb from a super-Chandrasekhar-mass white dwarf star", Nature, 443, 308-311 (2006). [DOI], [ADS]. (Cited on page 73.)

[292] Hulse, R. A. and Taylor, J. H., "Discovery of a pulsar in a binary system", Astrophys. J., 195, L51-L53 (1975). [DOI], [ADS]. (Cited on pages 14, 15, 42, and 57.)

[293] Hurley, J. R., Pols, O. R. and Tout, C. A., "Comprehensive analytic formulae for stellar evolution as a function of mass and metallicity", Mon. Not. R. Astron. Soc., 315, 543-569 (2000). [DOI], [ADS], [arXiv:astro-ph/0001295]. (Cited on page 29.)

[294] Hurley, J. R., Tout, C. A. and Pols, O. R., "Evolution of binary stars and the effect of tides on binary populations", Mon. Not. R. Astron. Soc., 329, 897-928 (2002). [DOI], [ADS], [arXiv:astroph/0201220]. (Cited on pages 46, 52, and 95.)

[295] Ibeling, D. and Heger, A., "The Metallicity Dependence of the Minimum Mass for Core-collapse Supernovae", Astrophys. J. Lett., 765, L43 (2013). [DOI], [ADS], [arXiv:1301.5783 [astro-ph.SR]]. (Cited on page 9.)

[296] Iben Jr, I., "Hot accreting white dwarfs in the quasi-static approximation", Astrophys. J., 259, 244-266 (1982). [DOI], [ADS]. (Cited on page 60.)

[297] Iben Jr, I., Stellar Evolution Physics, Volume 1: Physical Processes in Stellar Interiors, (Cambridge University Press, Cambridge; New York, 2013). [ADS]. (Cited on page 11.)

[298] Iben Jr, I., Stellar Evolution Physics, Volume 2: Advanced Evolution of Single Stars, (Cambridge University Press, Cambridge; New York, 2013). [ADS]. (Cited on page 11.)

[299] Iben Jr, I. and Livio, M., "Common envelopes in binary star evolution", Publ. Astron. Soc. Pac., 105, 1373-1406 (1993). [ADS]. (Cited on page 39.)

[300] Iben Jr, I. and Tutukov, A. V., "Supernovae of type I as end products of the evolution of binaries with components of moderate initial mass $\left(M \lesssim 9 M_{\odot}\right)$ ", Astrophys. J. Suppl. Ser., 54, 335-372 (1984). [DOI], [ADS]. (Cited on pages 28, 72, and 79.)

[301] Iben Jr, I. and Tutukov, A. V., "On the evolution of close binaries with components of initial mass between 3 solar masses and 12 solar masses", Astrophys. J. Suppl. Ser., 58, 661-710 (1985). (Cited on pages $10,60,64$, and 77.)

[302] Iben Jr, I. and Tutukov, A. V., "Helium star cataclysmics", Astrophys. J., 370, 615-629 (1991). [ADS]. (Cited on page 88.)

[303] Iben Jr, I. and Tutukov, A. V., "On the Evolution of Symbiotic Stars and Other Binaries with Accreting Degenerate Dwarfs", Astrophys. J. Suppl. Ser., 105, 145 (1996). [ADS]. (Cited on pages 60 and 68.)

[304] Iben Jr, I., Tutukov, A. V. and Fedorova, A. V., "On the Luminosity of White Dwarfs in Close Binaries Merging under the Influence of Gravitational Wave Radiation", Astrophys. J., 503, 344 (1998). [DOI], [ADS]. (Cited on page 94.)

[305] Iben Jr, I., Tutukov, A. V. and Yungelson, L. R., "A Model of the Galactic X-Ray Binary Population. II. Low-Mass X-Ray Binaries in the Galactic Disk", Astrophys. J. Suppl. Ser., 100, 233 (1995). (Cited on page 46.)

[306] Iben Jr, I., Tutukov, A. V. and Yungelson, L. R., "On the Origin of Hydrogen-deficient Supergiants and Their Relation to R Coronae Borealis Stars and Non-DA White Dwarfs", Astrophys. J., 456, 750 (1996). [DOI], [ADS]. (Cited on pages 64 and 76.) 
[307] Idan, I., Shaviv, N. J. and Shaviv, G., "The fate of a WD accreting H-rich material at high accretion rates", Mon. Not. R. Astron. Soc., 433, 2884-2892 (2013). [DOI], [ADS]. (Cited on pages 60 and 69.)

[308] Ilkov, M. and Soker, N., "Type Ia supernovae from very long delayed explosion of core-white dwarf merger", Mon. Not. R. Astron. Soc., 419, 1695-1700 (2012). [DOI], [ADS], [arXiv:1106.2027 [astroph.SR]]. (Cited on page 77.)

[309] in’t Zand, J. J. M., Jonker, P. G. and Markwardt, C. B., "Six new candidate ultracompact X-ray binaries", Astron. Astrophys., 465, 953-963 (2007). [DOI], [arXiv:astro-ph/0701810]. (Cited on page 79.)

[310] Isern, J., García-Berro, E. and Lorén-Aguilar, P., "Type Ia Supernovae and the DD Scenario", in Capuzzo-Dolcetta, R., Limongi, M. and Tornambè, A., eds., Advances in Computational Astrophysics: Methods, Tools, and Outcome, ASP Conference Series, 453, p. 99, (Astronomical Society of the Pacific, San Francisco, 2012). [ADS], [arXiv:1110.1856 [astro-ph.HE]]. (Cited on page 73.)

[311] Isern, J., Labay, J., Hernanz, M. and Canal, R., "Collapse and explosion of white dwarfs. I. Precollapse evolution", Astrophys. J., 273, 320-329 (1983). [DOI], [ADS]. (Cited on page 73.)

[312] Israel, G. L., Panzera, M. R., Campana, S., Lazzati, D., Covino, S., Tagliaferri, G. and Stella, L., "The discovery of $321 \mathrm{~S}$ pulsations in the ROSAT HRI light curves of 1BMW J080622.8+152732= RX J0806.3+1527", Astron. Astrophys., 349, L1-L4 (1999). [ADS]. (Cited on page 105.)

[313] Ivanova, N., "Common Envelope: On the Mass and the Fate of the Remnant", Astrophys. J., 730, 76 (2011). [DOI], [ADS], [arXiv:1101.2863 [astro-ph.SR]]. (Cited on pages 39 and 62.)

[314] Ivanova, N., Belczynski, K., Kalogera, V., Rasio, F. A. and Taam, R. E., "The Role of Helium Stars in the Formation of Double Neutron Stars", Astrophys. J., 592, 475-485 (2003). [DOI]. (Cited on page 46.)

[315] Ivanova, N. and Chaichenets, S., "Common Envelope: Enthalpy Consideration", Astrophys. J. Lett., 731, L36 (2011). [DOI], [ADS], [arXiv:1103.2790 [astro-ph.SR]]. (Cited on page 39.)

[316] Ivanova, N., Justham, S., Avendano Nandez, J. L. and Lombardi, J. C., "Identification of the Long-Sought Common-Envelope Events", Science, 339, 433 (2013). [DOI], [ADS], [arXiv:1301.5897 [astro-ph.HE]]. (Cited on page 41.)

[317] Ivanova, N., Rasio, F. A., Lombardi, J. C., Dooley, K. L. and Proulx, Z. F., "Formation of Ultracompact X-Ray Binaries in Dense Star Clusters", Astrophys. J. Lett., 621, L109-L112 (2005). [DOI], [ADS]. (Cited on page 78.)

[318] Ivanova, N. and Taam, R. E., "Thermal Timescale Mass Transfer and the Evolution of White Dwarf Binaries", Astrophys. J., 601, 1058-1066 (2004). [DOI], [ADS], [arXiv:astro-ph/0310126]. (Cited on pages 41 and 88.)

[319] Ivanova, N. et al., "Common envelope evolution: where we stand and how we can move forward", Astron. Astrophys. Rev., 21, 59 (2013). [DOI], [ADS], [arXiv:1209.4302 [astro-ph.HE]]. (Cited on pages 20,38, and 39.)

[320] Janka, H.-T., "Explosion Mechanisms of Core-Collapse Supernovae", Annu. Rev. Nucl. Part. Sci., 62, 407-451 (2012). [DOI], [ADS], [arXiv:1206.2503 [astro-ph.SR]]. (Cited on page 12.)

[321] Janka, H.-T., "Natal kicks of stellar mass black holes by asymmetric mass ejection in fallback supernovae", Mon. Not. R. Astron. Soc., 434, 1355-1361 (2013). [DOI], [ADS], [arXiv:1306.0007 [astroph.SR]]. (Cited on pages 35 and 48.)

[322] Ji, S. et al., "The Post-merger Magnetized Evolution of White Dwarf Binaries: The Doubledegenerate Channel of Sub-Chandrasekhar Type Ia Supernovae and the Formation of Magnetized White Dwarfs", Astrophys. J., 773, 136 (2013). [DOI], [arXiv:1302.5700]. (Cited on pages 7 and 76.) 
[323] Johansson, J., Woods, T. E., Gilfanov, M., Sarzi, M., Chen, Y.-M. and Oh, K., "Diffuse Gas in Galaxies Sheds New Light on the Origin of Type Ia Supernovae", arXiv, e-print, (2014). [ADS], [arXiv:1401.1344 [astro-ph.CO]]. (Cited on page 72.)

[324] Johnston, S., Hobbs, G., Vigeland, S. J., Kramer, M., Weisberg, J. M. and Lyne, A. G., "Evidence for alignment of the rotation and velocity vectors in pulsars", Mon. Not. R. Astron. Soc., 364, 1397-1412 (2005). [DOI], [arXiv:astro-ph/0510260]. (Cited on page 34.)

[325] Jones, S., Hirschi, R., Herwig, F., Paxton, B., Timmes, F. X. and Nomoto, K., "Progenitors of electron-capture supernovae", in Roming, P., Kawai, N. and Pian, E., eds., Death of Massive Stars: Supernovae and Gamma-Ray Burst, Proceedings of IAU Symposium 279, April 2011, Proc. IAU, 279, pp. 341-342, (Cambridge University Press, Cambridge, 2012). [DOI], [ADS]. (Cited on page 9.)

[326] Jones, S. et al., "Advanced Burning Stages and Fate of $8--10 M_{\odot}$ Stars", Astrophys. J., 772, 150 (2013). [DOI], [ADS], [arXiv:1306.2030 [astro-ph.SR]]. (Cited on page 9.)

[327] Jonker, P. G. and Nelemans, G., "The distances to Galactic low-mass X-ray binaries: Consequences for black hole luminosities and kicks", Mon. Not. R. Astron. Soc., 354, 355-366 (2004). [DOI], [ADS]. (Cited on page 35.)

[328] Justham, S., "Single-degenerate Type Ia Supernovae Without Hydrogen Contamination", Astrophys. J. Lett., 730, L34 (2011). [DOI], [ADS], [arXiv:1102.4913 [astro-ph.SR]]. (Cited on page 72.)

[329] Kahabka, P., "Recurrent supersoft X-ray sources", Astron. Astrophys., 304, 227 (1995). [ADS]. (Cited on page 61.)

[330] Kahabka, P. and van den Heuvel, E. P. J., "Luminous supersoft X-ray sources", Annu. Rev. Astron. Astrophys., 35, 69-100 (1997). [DOI]. (Cited on page 61.)

[331] Kalogera, V., "Spin-Orbit Misalignment in Close Binaries with Two Compact Objects", Astrophys. J., 541, 319-328 (2000). [DOI], [ADS]. (Cited on page 49.)

[332] Kalogera, V., Belczynski, K., Kim, C., O'Shaughnessy, R. and Willems, B., "Formation of double compact objects", Phys. Rep., 442, 75-108 (2007). [DOI], [ADS], [arXiv:astro-ph/0612144]. (Cited on page 46.)

[333] Kalogera, V., Narayan, R., Spergel, D. N. and Taylor, J. H., "The Coalescence Rate of Double Neutron Star Systems", Astrophys. J., 556, 340-356 (2001). [DOI], [astro-ph/0012038]. (Cited on page 17.)

[334] Kalogera, V. and Webbink, R. F., "Formation of Low-Mass X-Ray Binaries. I. Constraints on Hydrogen-rich Donors at the Onset of the X-Ray Phase", Astrophys. J., 458, 301-311 (1996). [DOI]. (Cited on page 46.)

[335] Kalogera, V. and Webbink, R. F., "Formation of Low-Mass X-Ray Binaries. II. Common Envelope Evolution of Primordial Binaries with Extreme Mass Ratios", Astrophys. J., 493, 351-368 (1998). [DOI]. (Cited on page 46.)

[336] Kalogera, V. et al., "The Cosmic Coalescence Rates for Double Neutron Star Binaries", Astrophys. J. Lett., 601, L179-L182 (2004). [DOI], [astro-ph/0312101]. (Cited on page 53.)

[337] Kaplan, D. L., Bildsten, L. and Steinfadt, J. D. R., "Orbital Evolution of Compact White Dwarf Binaries", Astrophys. J., 758, 64 (2012). [DOI], [ADS], [arXiv:1208.6320 [astro-ph.SR]]. (Cited on page 88.)

[338] Karakas, A. I., "Current Status of Stellar Evolutionary Models for AGB Stars", in Kerschbaum, F., Lebzelter, T. and Wing, R. F., eds., Why Galaxies Care about AGB Stars II: Shining Examples and Common Inhabitants, Proceedings of a conference held at University Campus, Vienna, Austria, 16-20 August 2010, ASP Conference Series, 445, pp. 3-12, (Astronomical Society of the Pacific, San Francisco, 2011). [ADS]. (Cited on page 9.) 
[339] Karl, C., Heber, U. and Napiwotzki, R., "Subdwarf B Binaries from the SPY Project", in Koester, D. and Moehler, S., eds., 14th European Workshop on White Dwarfs, Proceedings of a meeting held at Kiel, July 19-23, 2004, ASP Conference Series, 334, pp. 369-374, (Astronomical Society of the Pacific, San Francisco, 2005). [ADS]. (Cited on page 79.)

[340] Kasen, D., "Seeing the Collision of a Supernova with its Companion Star", Astrophys. J., 708, 1025-1031 (2010). [DOI], [arXiv:0909.0275 [astro-ph.HE]]. (Cited on pages 70 and 71.)

[341] Kashi, A. and Soker, N., "A circumbinary disc in the final stages of common envelope and the coredegenerate scenario for Type Ia supernovae", Mon. Not. R. Astron. Soc., 417, 1466-1479 (2011). [DOI], [ADS], [arXiv:1105.5698 [astro-ph.SR]]. (Cited on pages 38 and 62.)

[342] Kasliwal, M. M., "Systematically Bridging the Gap Between Novae and Supernovae", Publ. Astron. Soc. Australia, 29, 482-488 (2012). [DOI], [ADS]. (Cited on page 41.)

[343] Kasliwal, M. M. and Nissanke, S., "On Discovering Electromagnetic Emission from Neutron Star Mergers: The Early Years of Two Gravitational Wave Detectors", arXiv, e-print, (2013). [ADS], [arXiv:1309.1554 [astro-ph.HE]]. (Cited on page 56.)

[344] Kasliwal, M. M. et al., "Rapidly Decaying Supernova 2010X: A Candidate '.Ia' Explosion", Astrophys. J. Lett., 723, L98-L102 (2010). [DOI], [ADS], [arXiv:1009.0960 [astro-ph.HE]]. (Cited on page 91.)

[345] Kaspi, V. M., Bailes, M., Manchester, R. N., Stappers, B. W. and Bell, J. F., "Evidence from a precessing pulsar orbit for a neutron-star birth kick", Nature, 381, 584-586 (1996). [DOI]. (Cited on page 34 .)

[346] Kato, M. and Hachisu, I., "Optically thick winds in nova outbursts", Astrophys. J., 437, 802-826 (1994). [DOI], [ADS]. (Cited on pages 31 and 68.)

[347] Kato, M. and Hachisu, I., "A New Estimation of Mass Accumulation Efficiency in Helium Shell Flashes toward Type Ia Supernova Explosions", Astrophys. J. Lett., 513, L41-L44 (1999). [DOI], [ADS], [arXiv:astro-ph/9901080]. (Cited on page 67.)

[348] Kato, M. and Hachisu, I., "Mass Accumulation Efficiency in Helium Shell Flashes for Various White Dwarf Masses", Astrophys. J. Lett., 613, L129-L132 (2004). [DOI], [ADS]. (Cited on page 69.)

[349] Kato, M. and Hachisu, I., "Recurrent novae as progenitors of Type Ia supernovae", Bull. Astr. Soc. India, 40, 393 (2012). [ADS], [arXiv:1212.2295 [astro-ph.SR]]. (Cited on page 68.)

[350] Katz, B. and Dong, S., "The rate of WD-WD head-on collisions may be as high as the SNe Ia rate", arXiv, e-print, (2012). [ADS], [arXiv:1211.4584 [astro-ph.SR]]. (Cited on page 77.)

[351] Keane, E. F. and Kramer, M., "On the birthrates of Galactic neutron stars", Mon. Not. R. Astron. Soc., 391, 2009-2016 (2008). [DOI], [ADS], [arXiv:0810.1512]. (Cited on page 16.)

[352] Kelley, L. Z., Ramirez-Ruiz, E., Zemp, M., Diemand, J. and Mandel, I., "The Distribution of Coalescing Compact Binaries in the Local Universe: Prospects for Gravitational-wave Observations", Astrophys. J. Lett., 725, L91-L96 (2010). [DOI], [ADS], [arXiv:1011.1256 [astro-ph.HE]]. (Cited on page 35.)

[353] Kennicutt, R. C. and Evans, N. J., "Star Formation in the Milky Way and Nearby Galaxies", Annu. Rev. Astron. Astrophys., 50, 531-608 (2012). [DOI], [arXiv:1204.3552 [astro-ph.GA]]. (Cited on pages 9 and 17.)

[354] Kerzendorf, W. E., Childress, M., Scharwächter, J., Do, T. and Schmidt, B. P., "A Reconnaissance of the Possible Donor Stars to the Kepler Supernova", Astrophys. J., 782, 27 (2014). [DOI], [ADS], [arXiv:1309.5964 [astro-ph.SR]]. (Cited on page 70.) 
[355] Kerzendorf, W. E. et al., "A High-resolution Spectroscopic Search for the Remaining Donor for Tycho's Supernova", Astrophys. J., 774, 99 (2013). [DOI], [ADS], [arXiv:1210.2713]. (Cited on page 70.)

[356] Kiel, P. D., Hurley, J. R. and Bailes, M., "The long and the short of it: modelling double neutron star and collapsar Galactic dynamics", Mon. Not. R. Astron. Soc., 406, 656-672 (2010). [DOI], [ADS], [arXiv:1004.0131 [astro-ph.GA]]. (Cited on page 46.)

[357] Kiel, P. D., Hurley, J. R., Bailes, M. and Murray, J. R., "Populating the Galaxy with pulsars I. Stellar and binary evolution", Mon. Not. R. Astron. Soc., 388, 393-415 (2008). [DOI], [ADS], [arXiv:0805.0059]. (Cited on page 46.)

[358] Kilic, M., Brown, W. R. and Hermes, J. J., "Ultra-Compact Binaries: eLISA Verification Sources", in Auger, G., Binétruy, P. and Plagnol, E., eds., The 9th LISA Symposium, Bibliothéque Nationale de France, Paris, 21-25 May 2012, ASP Conference Series, 467, p. 47, (Astronomical Society of the Pacific, San Francisco, 2013). [ADS]. (Cited on pages 81 and 104.)

[359] Kilic, M. et al., "The shortest period detached binary white dwarf system", Mon. Not. R. Astron. Soc., 413, L101-L105 (2011). [DOI], [ADS], [arXiv:1103.2354 [astro-ph.GA]]. (Cited on pages 57 and 81.)

[360] Kilic, M. et al., "Found: the progenitors of AM CVn and supernovae .Ia", Mon. Not. R. Astron. Soc., 438, L26-L30 (2014). [DOI], [ADS], [arXiv:1310.6359 [astro-ph.GA]]. (Cited on page 81.)

[361] Kim, C., Kalogera, V. and Lorimer, D. R., "The probability distribution of binary pulsar coalescence rates. I. double neutron star systems in the galactic field", Astrophys. J., 584, 985-995 (2003). [DOI], [ADS], [arXiv:astro-ph/0207408 [astro-ph]]. (Cited on page 16.)

[362] Kim, C., Kalogera, V. and Lorimer, D. R., "Effect of PSR J0737-3039 on the DNS Merger Rate and Implications for GW Detection", arXiv, e-print, (2006). [ADS], [arXiv:astro-ph/0608280]. (Cited on page 16.)

[363] Kim, C., Perera, B. B. P. and McLaughlin, M. A., "Implications of PSR J0737-3039B for the Galactic NS-NS Binary Merger Rate", arXiv, e-print, (2013). [ADS], [arXiv:1308.4676 [astro-ph.SR]]. (Cited on pages 16 and 53.)

[364] King, A. R. and Begelman, M. C., "Radiatively Driven Outflows and Avoidance of Common-Envelope Evolution in Close Binaries", Astrophys. J. Lett., 519, L169-L171 (1999). [DOI]. (Cited on page 31.)

[365] King, A. R. and Ritter, H., "Cygnus X-2, super-Eddington mass transfer, and pulsar binaries", Mon. Not. R. Astron. Soc., 309, 253-260 (1999). [DOI], [ADS]. (Cited on page 40.)

[366] Kitaura, F. S., Janka, H.-T. and Hillebrandt, W., "Explosions of O-Ne-Mg cores, the Crab supernova, and subluminous type II-P supernovae", Astron. Astrophys., 450, 345-350 (2006). [DOI], [ADS]. (Cited on pages 10 and 34.)

[367] Kiziltan, B., Kottas, A., De Yoreo, M. and Thorsett, S. E., "The Neutron Star Mass Distribution", arXiv, e-print, (2013). [ADS], [arXiv:1309.6635 [astro-ph.SR]]. (Cited on page 8.)

[368] Knigge, C., Baraffe, I. and Patterson, J., "The Evolution of Cataclysmic Variables as Revealed by Their Donor Stars", Astrophys. J. Suppl. Ser., 194, 28 (2011). [DOI], [ADS], [arXiv:1102.2440 [astro-ph.SR]]. (Cited on page 26.)

[369] Knigge, C., Coe, M. J. and Podsiadlowski, P., "Two populations of X-ray pulsars produced by two types of supernova", Nature, 479, 372-375 (2011). [DOI], [ADS], [arXiv:1111.2051 [astro-ph.SR]]. (Cited on pages 10 and 42.)

[370] Kobulnicky, H. A. and Fryer, C. L., "A New Look at the Binary Characteristics of Massive Stars", Astrophys. J., 670, 747-765 (2007). [DOI], [ADS]. (Cited on page 12.) 
[371] Kochanek, C. S., "Failed Supernovae Explain the Compact Remnant Mass Function", arXiv, e-print, (2013). [ADS], [arXiv:1308.0013]. (Cited on pages 18 and 48.)

[372] Koester, D., Voss, B., Napiwotzki, R., Christlieb, N., Homeier, D., Lisker, T., Reimers, D. and Heber, U., "High-resolution UVES/VLT spectra of white dwarfs observed for the ESO SN Ia Progenitor Survey. III. DA white dwarfs", Astron. Astrophys., 505, 441-462 (2009). [DOI], [ADS]. (Cited on page 79.)

[373] Koester, D. et al., "High-resolution UVES/VLT spectra of white dwarfs observed for the ESO SN Ia progenitor survey (SPY). I.", Astron. Astrophys., 378, 556-568 (2001). [DOI]. (Cited on page 79.)

[374] Koliopanos, F., Gilfanov, M. and Bildsten, L., "X-ray diagnostics of chemical composition of the accretion disc and donor star in ultra-compact X-ray binaries", Mon. Not. R. Astron. Soc., 432, 1264-1273 (2013). [DOI], [ADS]. (Cited on page 78.)

[375] Kopal, Z., Close Binary Systems, The International Astrophysics Series, 5, (Chapman \& Hall; Wiley, London; New York, 1959). (Cited on pages 26 and 27.)

[376] Kopparapu, R. K., Hanna, C., Kalogera, V., O’Shaughnessy, R., González, G., Brady, P. R. and Fairhurst, S., "Host Galaxies Catalog Used in LIGO Searches for Compact Binary Coalescence Events", Astrophys. J., 675, 1459-1467 (2008). [DOI], [ADS], [arXiv:0706.1283]. (Cited on pages 17 and 55.)

[377] Kornilov, V. G. and Lipunov, V. M., "Neutron Stars in Massive Binary Systems - Part One Classification and Evolution", Sov. Astron., 27, 163 (1983). (Cited on page 51.)

[378] Kornilov, V. G. and Lipunov, V. M., "Neutron Stars in Massive Binary Systems - Part Two Numerical Modeling", Sov. Astron., 27, 334 (1983). (Cited on page 51.)

[379] Kotko, I., Lasota, J.-P., Dubus, G. and Hameury, J.-M., "Models of AM Canum Venaticorum star outbursts", Astron. Astrophys., 544, A13 (2012). [DOI], [ADS], [arXiv:1205.5999 [astro-ph.SR]]. (Cited on pages 85 and 106.)

[380] Kouwenhoven, M. B. N., The primordial binary population in the association Sco OB2, Ph.D. thesis, (University of Amsterdam, Amsterdam, 2006). [ADS]. (Cited on page 12.)

[381] Kouwenhoven, M. B. N., Brown, A. G. A., Goodwin, S. P., Portegies Zwart, S. F. and Kaper, L., "Exploring the consequences of pairing algorithms for binary stars", Astron. Astrophys., 493, 979-1016 (2009). [DOI], [arXiv:0811.2859]. (Cited on page 12.)

[382] Kouwenhoven, M. B. N., Brown, A. G. A., Portegies Zwart, S. F. and Kaper, L., "The primordial binary population. II. Recovering the binary population for intermediate mass stars in Scorpius OB2", Astron. Astrophys., 474, 77-104 (2007). [DOI], [ADS], [arXiv:0707.2746]. (Cited on page 12.)

[383] Kowalska, I., Bulik, T., Belczynski, K., Dominik, M. and Gondek-Rosinska, D., "The eccentricity distribution of compact binaries", Astron. Astrophys., 527, A70 (2011). [DOI], [ADS], [arXiv:1010.0511 [astro-ph.CO]]. (Cited on page 46.)

[384] Kraft, R. P., Mathews, J. and Greenstein, J. L., "Binary Stars among Cataclysmic Variables. II. Nova WZ Sagittae: A Possible Radiator of Gravitational Waves", Astrophys. J., 136, 312 (1962). [DOI], [ADS]. (Cited on page 57.)

[385] Kraus, A. L. and Hillenbrand, L. A., "Unusually Wide Binaries: Are They Wide or Unusual?", Astrophys. J., 703, 1511-1530 (2009). [DOI], [ADS], [arXiv:0908.1385 [astro-ph.SR]]. (Cited on page 12.)

[386] Kreidberg, L., Bailyn, C. D., Farr, W. M. and Kalogera, V., "Mass Measurements of Black Holes in X-Ray Transients: Is There a Mass Gap?", Astrophys. J., 757, 36 (2012). [DOI], [ADS], [arXiv:1205.1805 [astro-ph.HE]]. (Cited on page 18.) 
[387] Kromer, M., Sim, S. A., Fink, M., Röpke, F. K., Seitenzahl, I. R. and Hillebrandt, W., "Doubledetonation Sub-Chandrasekhar Supernovae: Synthetic Observables for Minimum Helium Shell Mass Models", Astrophys. J., 719, 1067-1082 (2010). [DOI], [ADS]. (Cited on page 76.)

[388] Kromer, M. et al., "SN 2010lp - A Type Ia Supernova from a Violent Merger of Two Carbon-Oxygen White Dwarfs", Astrophys. J. Lett., 778, L18 (2013). [DOI], [ADS], [arXiv:1311.0310 [astro-ph.HE]]. (Cited on page 76.)

[389] Krumholz, M. R., "The Big Problems in Star Formation: the Star Formation Rate, Stellar Clustering, and the Initial Mass Function", arXiv, e-print, (2014). [ADS], [arXiv:1402.0867 [astro-ph.GA]]. (Cited on page 9.)

[390] Kudritzki, R.-P. and Urbaneja, M. A., "Parameters and Winds of Hot Massive Stars", arXiv, e-print, (2006). [ADS], [arXiv:astro-ph/0607460]. (Cited on page 48.)

[391] Kuiper, G. P., "Problems of Double-Star Astronomy. I", Publ. Astron. Soc. Pac., 47, 15-42 (1935). [ADS]. (Cited on page 50.)

[392] Külebi, B., Ekşi, K. Y., Lorén-Aguilar, P., Isern, J. and García-Berro, E., "Magnetic white dwarfs with debris discs", Mon. Not. R. Astron. Soc., 431, 2778-2788 (2013). [DOI], [ADS], [arXiv:1302.6468 [astro-ph.SR]]. (Cited on page 7.)

[393] Külebi, B., Jordan, S., Nelan, E., Bastian, U. and Altmann, M., "Constraints on the origin of the massive, hot, and rapidly rotating magnetic white dwarf RE J 0317-853 from an HST parallax measurement", Astron. Astrophys., 524, A36 (2010). [DOI], [ADS], [arXiv:1007.4978 [astro-ph.SR]]. (Cited on page 76.)

[394] Kumar, S. S., "The Structure of Stars of Very Low Mass", Astrophys. J., 137, 1121 (1963). (Cited on page 8.)

[395] Kupfer, T., Groot, P. J., Levitan, D., Steeghs, D., Marsh, T. R., Rutten, R. G. M. and Nelemans, G., "Orbital periods and accretion disc structure of four AM CVn systems", Mon. Not. R. Astron. Soc., 432, 2048-2060 (2013). [DOI], [ADS], [arXiv:1303.5610 [astro-ph.SR]]. (Cited on page 79.)

[396] Kuranov, A. G., Popov, S. B. and Postnov, K. A., "Pulsar spin-velocity alignment from single and binary neutron star progenitors", Mon. Not. R. Astron. Soc., 395, 2087-2094 (2009). [DOI], [ADS], [arXiv:0901.1055 [astro-ph.SR]]. (Cited on pages 34 and 44.)

[397] Kuranov, A. G. and Postnov, K. A., "Neutron stars in globular clusters: Formation and observational manifestations", Astron. Lett., 32, 393-405 (2006). [DOI], [ADS], [arXiv:astro-ph/0605115]. (Cited on pages 10 and 34.)

[398] Kusenko, A., "Pulsar Kicks from Neutrino Oscillations", Int. J. Mod. Phys. D, 13, 2065-2084 (2004). [DOI]. (Cited on page 34.)

[399] Kushnir, D., Katz, B., Dong, S., Livne, E. and Fernández, R., "Head-on Collisions of White Dwarfs in Triple Systems Could Explain Type Ia Supernovae", Astrophys. J. Lett., 778, L37 (2013). [DOI], [ADS], [arXiv:1303.1180 [astro-ph.HE]]. (Cited on page 77.)

[400] Kuulkers, E. et al., "MAXI J1659-152: the shortest orbital period black-hole transient in outburst", Astron. Astrophys., 552, A32 (2013). [DOI], [ADS], [arXiv:1204.5840 [astro-ph.HE]]. (Cited on page 35.)

[401] Lai, D., "Neutron Star Kicks and Asymmetric Supernovae", in Blaschke, D., Glendenning, N. K. and Sedrakian, A., eds., Physics of Neutron Star Interiors, Lecture Notes in Physics, 578, p. 424, (Springer, Berlin; New York, 2001). [ADS]. (Cited on page 34.)

[402] Lai, D., Chernoff, D. F. and Cordes, J. M., "Pulsar Jets: Implications for Neutron Star Kicks and Initial Spins", Astrophys. J., 549, 1111-1118 (2001). [DOI]. (Cited on page 34.) 
[403] Lamb, J. B., Oey, M. S., Graus, A. S., Adams, F. C. and Segura-Cox, D. M., "The Initial Mass Function of Field OB Stars in the Small Magellanic Cloud", Astrophys. J., 763, 101 (2013). [DOI], [ADS], [arXiv:1212.1205 [astro-ph.GA]]. (Cited on page 9.)

[404] Landau, L. D. and Lifshitz, E. M., Mechanics, Course of Theoretical Physics, 1, (Pergamon Press, Oxford; New York, 1969), 2nd edition. (Cited on page 21.)

[405] Landau, L. D. and Lifshitz, E. M., The Classical Theory of Fields, Course of Theoretical Physics, 2, (Pergamon Press, Oxford; New York, 1975), 4th edition. [Google Books]. (Cited on pages 22 and 23.)

[406] Langer, N., "Presupernova Evolution of Massive Single and Binary Stars", Annu. Rev. Astron. Astrophys., 50, 107-164 (2012). [DOI], [ADS], [arXiv:1206.5443 [astro-ph.SR]]. (Cited on pages 11 and 12.)

[407] Langer, N., Norman, C. A., de Koter, A., Vink, J. S., Cantiello, M. and Yoon, S.-C., "Pair creation supernovae at low and high redshift", Astron. Astrophys., 475, L19-L23 (2007). [DOI], [ADS], [arXiv:0708.1970]. (Cited on page 11.)

[408] Larson, S. L., Hiscock, W. A. and Hellings, R. W., "Sensitivity curves for spaceborne gravitational wave interferometers", Phys. Rev. D, 62, 062001 (2000). [DOI], [ADS]. (Cited on page 98.)

[409] Lasota, J.-P., "The disc instability model of dwarf novae and low-mass X-ray binary transients", New Astron. Rev., 45, 449-508 (2001). [DOI], [ADS], [arXiv:astro-ph/0102072]. (Cited on pages 18 and 60.)

[410] Lattimer, J. M., "Neutron star equation of state", New Astron. Rev., 54, 101-109 (2010). [DOI], [ADS]. (Cited on page 8.)

[411] Lattimer, J. M., "The Nuclear Equation of State and Neutron Star Masses", Annu. Rev. Nucl. Sci., 62, 485-515 (2012). [DOI]. (Cited on page 14.)

[412] Lau, H. H. B., Gil-Pons, P., Doherty, C. and Lattanzio, J., "The end of super AGB and massive AGB stars. I. The instabilities that determine the final mass of AGB stars", Astron. Astrophys., 542, A1 (2012). [DOI], [ADS], [arXiv:1204.3931 [astro-ph.SR]]. (Cited on pages 9 and 10.)

[413] Lentz, E. J., Baron, E., Hauschildt, P. H. and Branch, D., "Detectability of Hydrogen Mixing in Type Ia Supernova Premaximum Spectra", Astrophys. J., 580, 374-379 (2002). [DOI]. (Cited on page 69.)

[414] Lepo, K. and van Kerkwijk, M., "A Search for Rapidly Accreting White Dwarfs in the Small Magellanic Cloud", Astrophys. J., 771, 13 (2013). [DOI], [ADS], [arXiv:1301.1368 [astro-ph.SR]]. (Cited on page 68.)

[415] Levitan, D. et al., "Five new outbursting AM CVn systems discovered by the Palomar Transient Factory", Mon. Not. R. Astron. Soc., 430, 996-1007 (2013). [DOI], [ADS], [arXiv:1212.5312 [astroph.SR]]. (Cited on page 79.)

[416] Li, L.-X. and Paczyński, B., "Transient Events from Neutron Star Mergers", Astrophys. J. Lett., 507, L59-L62 (1998). [DOI], [ADS], [arXiv:astro-ph/9807272]. (Cited on pages 56 and 109.)

[417] Li, W., Chornock, R., Leaman, J., Filippenko, A. V., Poznanski, D., Wang, X., Ganeshalingam, M. and Mannucci, F., "Nearby supernova rates from the Lick Observatory Supernova Search - III. The rate-size relation, and the rates as a function of galaxy Hubble type and colour", Mon. Not. R. Astron. Soc., 412, 1473-1507 (2011). [DOI], [ADS], [arXiv:1006.4613 [astro-ph.SR]]. (Cited on page 44.)

[418] Li, W., Filippenko, A. V., Treffers, R. R., Riess, A. G., Hu, J. and Qiu, Y., "A High Intrinsic Peculiarity Rate among Type Ia Supernovae", Astrophys. J., 546, 734-743 (2001). [DOI], [ADS]. (Cited on page 77.) 
[419] Li, Z., Mao, C., Chen, L., Zhang, Q. and Li, M., "The Potential Importance of Binary Evolution in Ultraviolet-Optical Spectral Fitting of Early-type Galaxies", Astrophys. J., 776, 37 (2013). [DOI], [ADS], [arXiv:1302.0927 [astro-ph.GA]]. (Cited on page 46.)

[420] Liebert, J., Bergeron, P. and Holberg, J. B., "The Formation Rate and Mass and Luminosity Functions of DA White Dwarfs from the Palomar Green Survey", Astrophys. J. Suppl. Ser., 156, 47-68 (2005). [DOI], [ADS], [astro-ph/0406657]. (Cited on page 50.)

[421] Linden, T., Kalogera, V., Sepinsky, J. F., Prestwich, A., Zezas, A. and Gallagher, J. S., "The Effect of Starburst Metallicity on Bright X-ray Binary Formation Pathways", Astrophys. J., 725, 1984-1994 (2010). [DOI], [ADS], [arXiv:1005.1639 [astro-ph.CO]]. (Cited on page 48.)

[422] Linden, T., Valsecchi, F. and Kalogera, V., "On the Rarity of X-Ray Binaries with Naked Helium Donors", Astrophys. J., 748, 114 (2012). [DOI], [ADS], [arXiv:1111.5012 [astro-ph.GA]]. (Cited on page 45.)

[423] Lipunov, V. M., Astrophysics of Neutron Stars, (Springer, Berlin; New York, 1992). (Cited on page 45 .)

[424] Lipunov, V. M., Bogomazov, A. I. and Abubekerov, M. K., "How abundant is the population of binary radio pulsars with black holes?", Mon. Not. R. Astron. Soc., 359, 1517-1523 (2005). [DOI]. (Cited on page 109.)

[425] Lipunov, V. M., Nazin, S. N., Panchenko, I. E., Postnov, K. A. and Prokhorov, M. E., "The gravitational wave sky", Astron. Astrophys., 298, 677 (1995). [ADS]. (Cited on page 55.)

[426] Lipunov, V. M. and Postnov, K. A., "Spectrum of gravitational radiation of binary systems", Sov. Astron., 31, 228-235 (1988). (Cited on pages 92 and 93.)

[427] Lipunov, V. M., Postnov, K. A. and Prokhorov, M. E., "The sources of gravitational waves with continuous and discrete spectra", Astron. Astrophys., 176, L1-L4 (1987). (Cited on page 93.)

[428] Lipunov, V. M., Postnov, K. A. and Prokhorov, M. E., "The Scenario Machine: Binary Star Population Synthesis", Astrophys. Space Phys. Rev., 9, 1-178 (1996). Online version (accessed 13 November 2006):

http://xray.sai.msu.ru/ mystery/articles/review/. (Cited on page 51.)

[429] Lipunov, V. M., Postnov, K. A. and Prokhorov, M. E., "Black Holes and Gravitational Waves: Possibilities for Simultaneous Detection Using First-Generation Laser Interferometers", Astron. Lett., 23, 492-497 (1997). [ADS]. (Cited on pages 52 and 55.)

[430] Lipunov, V. M., Postnov, K. A. and Prokhorov, M. E., "Formation and coalescence of relativistic binary stars: The effect of kick velocity", Mon. Not. R. Astron. Soc., 288, 245-259 (1997). [DOI]. (Cited on pages 35, 46, and 49.)

[431] Lipunov, V. M., Postnov, K. A., Prokhorov, M. E. and Osminkin, E. Y., "Binary Radiopulsars with Black Holes", Astrophys. J. Lett., 423, L121-L124 (1994). [DOI], [ADS]. (Cited on pages 18 and 109.)

[432] Lipunov, V. M., Postnov, K. A., Prokhorov, M. E., Panchenko, I. E. and Jørgensen, H. E., "Evolution of the Double Neutron Star Merging Rate and the Cosmological Origin of Gamma-Ray Burst Sources", Astrophys. J., 454, 593 (1995). [DOI], [ADS]. (Cited on page 55.)

[433] Littenberg, T. B., Larson, S. L., Nelemans, G. and Cornish, N. J., "Prospects for observing ultracompact binaries with space-based gravitational wave interferometers and optical telescopes", Mon. Not. R. Astron. Soc., 429, 2361-2365 (2013). [DOI], [ADS], [arXiv:1207.4848 [astro-ph.IM]]. (Cited on page 103.) 
[434] Littlefair, S. P., Dhillon, V. S., Marsh, T. R., Gänsicke, B. T., Southworth, J., Baraffe, I., Watson, C. A. and Copperwheat, C. M., "On the evolutionary status of short-period cataclysmic variables", Mon. Not. R. Astron. Soc., 388, 1582-1594 (2008). [DOI], [ADS], [arXiv:0806.1129]. (Cited on page 26.)

[435] Liu, Z.-W., Pakmor, R., Röpke, F. K., Edelmann, P., Hillebrandt, W., Kerzendorf, W. E., Wang, B. and Han, Z. W., "Rotation of surviving companion stars after type Ia supernova explosions in the WD+MS scenario", Astron. Astrophys., 554, A109 (2013). [DOI], [ADS], [arXiv:1303.2691 [astro-ph.SR]]. (Cited on page 70.)

[436] Liu, Z. W., Pakmor, R., Röpke, F. K., Edelmann, P., Wang, B., Kromer, M., Hillebrandt, W. and Han, Z. W., "Three-dimensional simulations of the interaction between Type Ia supernova ejecta and their main sequence companions", Astron. Astrophys., 548, A2 (2012). [DOI], [ADS], [arXiv:1209.4458 [astro-ph.SR]]. (Cited on page 69.)

[437] Livio, M. and Riess, A. G., "Have the Elusive Progenitors of Type Ia Supernovae Been Discovered?", Astrophys. J. Lett., 594, L93-L94 (2003). [DOI], [ADS], [arXiv:astro-ph/0308018]. (Cited on pages 62 and 77 .)

[438] Livio, M. and Soker, N., "The common envelope phase in the evolution of binary stars", Astrophys. J., 329, 764-779 (1988). [DOI], [ADS]. (Cited on page 38.)

[439] Livne, E., "Successive detonations in accreting white dwarfs as an alternative mechanism for type I supernovae", Astrophys. J., 354, L53-L55 (1990). [DOI], [ADS]. (Cited on page 73.)

[440] Livne, E. and Glasner, A., "Numerical simulations of off-center detonations in helium shells", Astrophys. J., 370, 272-281 (1991). [DOI]. (Cited on page 73.)

[441] Lombardi Jr, J. C., Proulx, Z. F., Dooley, K. L., Theriault, E. M., Ivanova, N. and Rasio, F. A., "Stellar Collisions and Ultracompact X-Ray Binary Formation", Astrophys. J., 640, 441-458 (2006). [DOI], [ADS], [arXiv:astro-ph/0509511]. (Cited on page 78.)

[442] Lommen, D., Yungelson, L. R., van den Heuvel, E., Nelemans, G. and Portegies Zwart, S. F., "Cygnus X-3 and the problem of the missing Wolf-Rayet X-ray binaries", Astron. Astrophys., 443, 231-241 (2005). [DOI], [ADS]. (Cited on pages 31 and 45.)

[443] Lorén-Aguilar, P., Guerrero, J., Isern, J., Lobo, J. A. and García-Berro, E., "Gravitational wave radiation from the coalescence of white dwarfs", Mon. Not. R. Astron. Soc., 356, 627-636 (2005). [DOI], [ADS]. (Cited on page 95.)

[444] Lorimer, D. R., "Binary and Millisecond Pulsars", Living Rev. Relativity, 11, lrr-2008-8 (2008). [DOI], [ADS], [arXiv:0811.0762]. URL (accessed 14 March 2014): http://www.livingreviews.org//rr-2008-8. (Cited on page 14.)

[445] Lorimer, D. R. et al., "Arecibo Pulsar Survey Using ALFA. II. The Young, Highly Relativistic Binary Pulsar J1906+0746", Astrophys. J., 640, 428-434 (2006). [DOI], [arXiv:astro-ph/0511523]. (Cited on page 15.)

[446] Loveridge, A. J., van der Sluys, M. V. and Kalogera, V., "Analytical Expressions for the Envelope Binding Energy of Giants as a Function of Basic Stellar Parameters", Astrophys. J., 743, 49 (2011). [DOI], [ADS], [arXiv:1009.5400 [astro-ph.SR]]. (Cited on page 39.)

[447] Lü, G., Yungelson, L. R. and Han, Z., "Population synthesis for symbiotic stars with white dwarf accretors", Mon. Not. R. Astron. Soc., 372, 1389-1406 (2006). [DOI], [ADS]. (Cited on page 67.)

[448] Lubow, S. H. and Shu, F. H., "Gas dynamics of semidetached binaries", Astrophys. J., 198, 383 (1975). [DOI]. (Cited on pages 27 and 106.) 
[449] Lyne, A. G. and Lorimer, D. R., "High Birth Velocities of Radio Pulsars", Nature, 369, 127-129 (1994). [DOI]. (Cited on page 33.)

[450] Lyne, A. G. et al., "The Parkes Multibeam Pulsar Survey: PSR J1811-1736, a pulsar in a highly eccentric binary system", Mon. Not. R. Astron. Soc., 312, 698-702 (2000). [DOI]. (Cited on page 15.)

[451] Lyne, A. G. et al., "A Double-Pulsar System: A Rare Laboratory for Relativistic Gravity and Plasma Physics", Science, 303, 1153-1157 (2004). [DOI], [arXiv:astro-ph/0401086]. (Cited on pages 14 and 15.)

[452] Maccarone, T. J., Lehmer, B. D., Leyder, J. C., Antoniou, V., Hornschemeier, A., Ptak, A., Wik, D. and Zezas, A., "A new candidate Wolf-Rayet X-ray binary in NGC 253", Mon. Not. R. Astron. Soc., 439, 3064-3072 (2014). [DOI], [ADS], [arXiv:1401.5425 [astro-ph.HE]]. (Cited on pages 18 and 19.)

[453] Madau, P., "Galaxy Evolution and the Cosmic Rate of Supernovae", in D'Odorico, S., Fontana, A. and Giallongo, E., eds., The Young Universe: Galaxy Formation and Evolution at Intermediate and High Redshift, Meeting at the Rome Astronomical Observatory, Monteporzio, Italy, 29 September3 October 1997, ASP Conference Series, 146, p. 289, (Astronomical Society of the Pacific, San Francisco, 1998). [ADS]. (Cited on page 7.)

[454] Mahy, L., Rauw, G., De Becker, M., Eenens, P. and Flores, C. A., "A spectroscopic investigation of the O-type star population in four Cygnus OB associations. I. Determination of the binary fraction", Astron. Astrophys., 550, A27 (2013). [DOI], [ADS], [arXiv:1301.0500 [astro-ph.SR]]. (Cited on page 12.)

[455] Manchester, R. N. et al., "The Parkes multi-beam pulsar survey - I. Observing and data analysis systems, discovery and timing of 100 pulsars", Mon. Not. R. Astron. Soc., 328, 17-35 (2001). [DOI]. (Cited on page 8.)

[456] Mandel, I. and O'Shaughnessy, R., "Compact binary coalescences in the band of groundbased gravitational-wave detectors", Class. Quantum Grav., 27, 114007 (2010). [DOI], [ADS], [arXiv:0912.1074 [astro-ph.HE]]. (Cited on page 8.)

[457] Maoz, D. and Mannucci, F., "Type-Ia Supernova Rates and the Progenitor Problem: A Review", Publ. Astron. Soc. Australia, 29, 447-465 (2012). [DOI], [ADS], [arXiv:1111.4492 [astro-ph.CO]]. (Cited on page 7.)

[458] Maoz, D., Mannucci, F. and Nelemans, G., "Observational clues to the progenitors of Type-Ia supernovae", arXiv, e-print, (2013). [ADS], [arXiv:1312.0628 [astro-ph.CO]]. (Cited on pages 7 and 77.)

[459] Marsh, T.R, "Detached double white dwarfs as AM CVn progenitors", Third International Workshop on AM CVn stars, University of Warwick, April 16-20, 2012, conference paper, (2012). Online version (accessed 17 March 2014):

http://www2.warwick.ac.uk/fac/sci/physics/research/astro/amcvn3/program/. (Cited on page 82.)

[460] Marsh, T. R., "Detached white-dwarf close-binary stars - CV's extended family", New Astron. Rev., 44, 119-124 (2000). [DOI], [ADS]. (Cited on page 79.)

[461] Marsh, T. R., "Double white dwarfs and LISA", Class. Quantum Grav., 28, 094019 (2011). [DOI], [ADS], [arXiv:1101.4970 [astro-ph.SR]]. (Cited on pages 62 and 63.)

[462] Marsh, T. R., Dhillon, V. S. and Duck, S. R., "Low-mass white dwarfs need friends: Five new double-degenerate close binary stars", Mon. Not. R. Astron. Soc., 275, 828 (1995). (Cited on page 79.)

[463] Marsh, T. R., Horne, K. and Rosen, S., "Evidence for CNO processed material in the accretion disk of GP Comae", Astrophys. J., 366, 535-543 (1991). [DOI], [ADS]. (Cited on page 82.) 
[464] Marsh, T. R., Nelemans, G. and Steeghs, D., "Mass transfer between double white dwarfs", Mon. Not. R. Astron. Soc., 350, 113-128 (2004). [DOI], [ADS], [arXiv:astro-ph/0312577]. (Cited on pages $73,74,84,95$, and 105.)

[465] Marsh, T. R. and Steeghs, D., "V407 Vul: A direct impact accretor", Mon. Not. R. Astron. Soc., 331, L7-L11 (2002). [DOI], [ADS]. (Cited on page 106.)

[466] Maselli, A. and Ferrari, V., "Low latency search for Gravitational waves from BH-NS binaries in coincidence with Short Gamma Ray Bursts", arXiv, e-print, (2013). [ADS], [arXiv:1312.5133 [grqc]]. (Cited on page 56.)

[467] Masevich, A. G., Tutukov, A. V. and Yungelson, L. R., "Gravitational radiation and the evolution of dwarf binaries", Priroda, 68-76 (1981). [ADS]. In Russian. (Cited on page 92.)

[468] Mason, B. D., Gies, D. R., Hartkopf, W. I., Bagnuolo Jr., W. G., ten Brummelaar, T. and McAlister, H. A., "ICCD speckle observations of binary stars. XIX. An astrometric/spectroscopic survey of O stars", Astron. J., 115, 821 (1998). [DOI], [ADS]. (Cited on page 12.)

[469] Mason, B. D., Hartkopf, W. I., Gies, D. R., Henry, T. J. and Helsel, J. W., "The High Angular Resolution Multiplicity of Massive Stars", Astron. J., 137, 3358-3377 (2009). [DOI], [ADS], [arXiv:0811.0492]. (Cited on page 12.)

[470] Massevich, A. G., Popova, E. I., Tutukov, A. V. and Yungelson, L. R., "On the influence of mass loss and convective overshooting on the evolution of massive stars", Astrophys. Space Sci., 62, 451-463 (1979). (Cited on page 46.)

[471] Massevitch, A. and Yungelson, L. R., "On the evolution of close binaries with mass and momentum loss from the system", Mem. Soc. Astron. Ital., 46, 217-229 (1975). [ADS]. (Cited on page 28.)

[472] Massevitch, A. G., Tutukov, A. V. and Yungelson, L. R., "Evolution of massive close binaries and formation of neutron stars and black holes", Astrophys. Space Sci., 40, 115-133 (1976). [DOI]. (Cited on page 42.)

[473] Maund, J. R. et al., "Spectropolarimetry of the Type Ia supernova 2012fr"", Mon. Not. R. Astron. Soc., 433, L20-L24 (2013). [DOI], [ADS], [arXiv:1302.0166 [astro-ph.SR]]. (Cited on page 72.)

[474] Maxted, P. F. L. et al., "EL CVn-type binaries - discovery of 17 helium white dwarf precursors in bright eclipsing binary star systems", Mon. Not. R. Astron. Soc., 437, 1681-1697 (2014). [DOI], [ADS], [arXiv:1310.4863 [astro-ph.SR]]. (Cited on page 81.)

[475] McClintock, J. E. and Remillard, R. A., "Black hole binaries", in Lewin, W. H. G. and van der Klis, M., eds., Compact Stellar X-Ray Sources, Cambridge Astrophysics Series, 39, pp. 157-214, (Cambridge University Press, Cambridge; New York, 2006). [DOI], [ADS], [arXiv:astro-ph/0306213], [Google Books]. (Cited on page 48.)

[476] McClintock, J. E. et al., "Measuring the spins of accreting black holes", Class. Quantum Grav., 28, 114009 (2011). [DOI], [ADS], [arXiv:1101.0811 [astro-ph.HE]]. (Cited on page 17.)

[477] Meng, X. and Podsiadlowski, P., "Constraining the Spin-down Timescale of the White Dwarf Progenitors of Type Ia Supernovae", Astrophys. J. Lett., 778, L35 (2013). [DOI], [ADS], [arXiv:1311.0166 [astro-ph.SR]]. (Cited on page 72.)

[478] Mengel, J. G., Norris, J. and Gross, P. G., "Binary Hypothesis for the Subdwarf B Stars", Astrophys. J., 204, 488-492 (1976). [DOI], [ADS]. (Cited on page 60.)

[479] Mennekens, N. and Vanbeveren, D., "Massive double compact object mergers: gravitational wave sources and r-process-element production sites", arXiv, e-print, (2013). [ADS], [arXiv:1307.0959]. (Cited on pages 52 and 55.) 
[480] Menon, A., Herwig, F., Denissenkov, P. A., Clayton, G. C., Staff, J., Pignatari, M. and Paxton, B., "Reproducing the Observed Abundances in RCB and HdC Stars with Post-double-degenerate Merger Models-Constraints on Merger and Post-merger Simulations and Physics Processes", Astrophys. J., 772, 59 (2013). [DOI], [ADS], [arXiv:1211.3392 [astro-ph.SR]]. (Cited on page 64.)

[481] Mestel, L., "On the theory of white dwarf stars. I. The energy sources of white dwarfs", Mon. Not. R. Astron. Soc., 112, 583 (1952). [ADS]. (Cited on page 60.)

[482] Mestel, L., "On the theory of white dwarf stars. II. The accretion of insterstellar matter by white dwarfs", Mon. Not. R. Astron. Soc., 112, 598 (1952). [ADS]. (Cited on page 60.)

[483] Metzger, B. D. et al., "Electromagnetic counterparts of compact object mergers powered by the radioactive decay of $r$-process nuclei", Mon. Not. R. Astron. Soc., 406, 2650-2662 (2010). [DOI], [ADS], [arXiv:1001.5029 [astro-ph.HE]]. (Cited on page 56.)

[484] Miller-Jones, J. C. A., Jonker, P. G., Dhawan, V., Brisken, W., Rupen, M. P., Nelemans, G. and Gallo, E., "The First Accurate Parallax Distance to a Black Hole", Astrophys. J. Lett., 706, L230L234 (2009). [DOI], [ADS], [arXiv:0910.5253 [astro-ph.HE]]. (Cited on page 35.)

[485] Mirabel, I. F. and Rodrigues, I., "Formation of a Black Hole in the Dark", Science, 300, 1119-1121 (2003). [DOI], [ADS], [arXiv:astro-ph/0305205]. (Cited on page 35.)

[486] Mirabel, I. F. and Rodríguez, L. F., "Sources of Relativistic Jets in the Galaxy", Annu. Rev. Astron. Astrophys., 37, 409-443 (1999). [DOI], [ADS], [arXiv:astro-ph/9902062]. (Cited on page 17.)

[487] Mironovskii, V. N., "Gravitational Radiation of Double Stars", Sov. Astron., 9, 752 (1965). [ADS]. (Cited on page 92.)

[488] Miyaji, S., Nomoto, K., Yokoi, K. and Sugimoto, D., "Supernova Triggered by Electron Captures", Publ. Astron. Soc. Japan, 32, 303 (1980). [ADS]. (Cited on pages 9 and 10.)

[489] Mochkovitch, R. and Livio, M., "The coalescence of white dwarfs and type I supernovae. The merged configuration", Astron. Astrophys., 236, 378-384 (1990). [ADS]. (Cited on page 73.)

[490] Mohamed, S. and Podsiadlowski, P., "Mass Transfer in Mira-type Binaries", Baltic Astron., 21, 88-96 (2012). [ADS]. (Cited on page 58.)

[491] Moll, R., Raskin, C., Kasen, D. and Woosley, S., "Type Ia Supernovae from Merging White Dwarfs I. Prompt Detonations", arXiv, e-print, (2013). [ADS], [arXiv:1311.5008 [astro-ph.HE]]. (Cited on page 77.$)$

[492] Moll, R. and Woosley, S. E., "Multi-dimensional Models for Double Detonation in SubChandrasekhar Mass White Dwarfs", Astrophys. J., 774, 137 (2013). [DOI]. (Cited on page 74.)

[493] Moore, K., Townsley, D. M. and Bildsten, L., "The Effects of Curvature and Expansion on Helium Detonations on White Dwarf Surfaces", Astrophys. J., 776, 97 (2013). [DOI], [ADS], [arXiv:1308.4193 [astro-ph.SR]]. (Cited on page 91.)

[494] Morrison, R. and McCammon, D., "Interstellar photoelectric absorption cross sections, 0.03-10 keV", Astrophys. J., 270, 119-122 (1983). [DOI], [ADS]. (Cited on page 106.)

[495] Motch, C., Haberl, F., Guillout, P., Pakull, M., Reinsch, K. and Krautter, J., "New cataclysmic variables from the ROSAT All-Sky Survey", Astron. Astrophys., 307, 459-469 (1996). [ADS]. (Cited on page 105.)

[496] Motl, P. M., Frank, J., Tohline, J. E. and D'Souza, M. C. R., "The Stability of Double White Dwarf Binaries Undergoing Direct-Impact Accretion", Astrophys. J., 670, 1314-1325 (2007). [DOI], [ADS], [arXiv:astro-ph/0702388]. (Cited on page 73.) 
[497] Müller, B. et al., "The Status of Core-Collapse Supernova Explosion Modelling", Supernovae and Gamma-Ray Bursts, YIPQS, Kyoto, Japan, October 14-November 15, 2013, conference paper, (2013). Online version (accessed 2 March 2014):

http://www2 yukawa.kyoto-u.ac.jp/ws/2013/sngrb/Program-Conferences.html. (Cited on page 48.)

[498] Munar-Adrover, P., Paredes, J. M., Ribó, M., Iwasawa, K., Zabalza, V. and Casares, J., "Discovery of X-Ray Emission from the First Be/Black Hole System", Astrophys. J. Lett., 786, L11 (2014). [DOI], [ADS], [arXiv:1404.0901 [astro-ph.HE]]. (Cited on page 19.)

[499] Muno, M. P. et al., "A Neutron Star with a Massive Progenitor in Westerlund 1", Astrophys. J. Lett., 636, L41-L44 (2006). [DOI], [ADS]. (Cited on page 48.)

[500] Nakar, E., "Short-hard gamma-ray bursts", Phys. Rep., 442, 166-236 (2007). [DOI], [ADS], [arXiv:astro-ph/0701748]. (Cited on pages 55 and 109.)

[501] Napiwotzki, R. et al., "Search for progenitors of supernovae type Ia with SPY", Astron. Nachr., 322, 411-418 (2001). [DOI]. (Cited on page 79.)

[502] Napiwotzki, R. et al., "SPY - The Eso Supernovae Type Ia Progenitor Survey", Messenger, 2003(112), 25-30 (2003). [ADS]. (Cited on page 79.)

[503] Napiwotzki, R. et al., "Binary White Dwarfs in the Supernova Ia Progenitor Survey", in Napiwotzki, R. and Burleigh, M. R., eds., 15th European Workshop on White Dwarfs, Proceedings of the conference held 7-11 August, 2006 in Leicester, United Kingdom, ASP Conference Series, 372, p. 387, (Astronomical Society of the Pacific, San Francisco, 2007). [ADS]. (Cited on page 79.)

[504] Narayan, R., "The birthrate and initial spin period of single radio pulsars", Astrophys. J., 319, 162-179 (1987). [DOI]. (Cited on page 16.)

[505] Narayan, R., Piran, T. and Shemi, A., "Neutron star and black hole binaries in the Galaxy", Astrophys. J. Lett., 379, L17-L20 (1991). [DOI], [ADS]. (Cited on pages 8 and 16.)

[506] Nauenberg, M., "Analytic Approximations to the Mass-Radius Relation and Energy of ZeroTemperature Stars", Astrophys. J., 175, 417 (1972). [DOI]. (Cited on page 85.)

[507] Nebot Gómez-Morán, A. et al., "Post common envelope binaries from SDSS. XII. The orbital period distribution", Astron. Astrophys., 536, A43 (2011). [DOI], [ADS], [arXiv:1109.6662 [astro-ph.SR]]. (Cited on page 60.)

[508] Nelemans, G., "Astrophysics of white dwarf binaries", in Merkovitz, S. M. and Livas, J. C., eds., Laser Interferometer Space Antenna: 6th International LISA Symposium, 6th International LISA Symposium, Greenbelt, MD, USA, $19-23$ June 2006, AIP Conference Proceedings, 873, pp. 397405, (American Institute of Physics, Melville, NY, 2006). [DOI], [ADS], [arXiv:astro-ph/0703292 [astro-ph]]. (Cited on page 105.)

[509] Nelemans, G., "The Galactic gravitational wave foreground", Class. Quantum Grav., 26, 094030 (2009). [DOI], [ADS], [arXiv:0901.1778 [astro-ph.SR]]. (Cited on pages 103, 104, and 105.)

[510] Nelemans, G., "Population synthesis of Galactic subdwarf B stars", Astrophys. Space Sci., 329, 25-31 (2010). [DOI], [ADS]. (Cited on page 60.)

[511] Nelemans, G., "Galactic Binaries with eLISA", in Auger, G., Binétruy, P. and Plagnol, E., eds., The 9th LISA Symposium, Bibliothéque Nationale de France, Paris, 21 - 25 May 2012, ASP Conference Series, 467, p. 27, (Astronomical Society of the Pacific, San Francisco, 2013). [ADS], [arXiv:1302.0138 [astro-ph.HE]]. (Cited on pages 58 and 105.)

[512] Nelemans, G., Portegies Zwart, S. F., Verbunt, F. and Yungelson, L. R., "Population synthesis for double white dwarfs. II. Semi-detached systems: AM CVn stars", Astron. Astrophys., 368, 939-949 (2001). [DOI], [ADS], [arXiv:astro-ph/0101123]. (Cited on pages 85, 86, 94, and 95.) 
[513] Nelemans, G., Steeghs, D. and Groot, P. J., "Spectroscopic evidence for the binary nature of AM CVn", Mon. Not. R. Astron. Soc., 326, 621-627 (2001). [DOI], [ADS]. (Cited on page 57.)

[514] Nelemans, G., Tauris, T. M. and van den Heuvel, E. P. J., "Constraints on mass ejection in black hole formation derived from black hole X-ray binaries", Astron. Astrophys., 352, L87-L90 (1999). (Cited on page 35.)

[515] Nelemans, G. and Tout, C. A., "Constraints on AM CVn formation channels from modelling the composition of their discs", in de Martino, D., Silvotti, R., Solheim, J.-E. and Kalytis, R., eds., NATO ASIB Proc. 105: White Dwarfs, Proceedings of the conference held at the Astronomical Observatory of Capodimonte, Napoli, Italy, NATO ASI Series II, 105, p. 359, (Kluwer, Dordrecht; Boston, 2003). [ADS]. (Cited on pages 78 and 89.)

[516] Nelemans, G. and Tout, C. A., "Reconstructing the evolution of white dwarf binaries: Further evidence for an alternative algorithm for the outcome of the common-envelope phase in close binaries", Mon. Not. R. Astron. Soc., 356, 753-764 (2005). [DOI], [ADS]. (Cited on page 40.)

[517] Nelemans, G., Verbunt, F., Yungelson, L. R. and Portegies Zwart, S. F., "Reconstructing the evolution of double helium white dwarfs: Envelope loss without spiral-in", Astron. Astrophys., 360, 1011-1018 (2000). [ADS], [arXiv:astro-ph/0006216]. (Cited on pages 39 and 40.)

[518] Nelemans, G., Yungelson, L. R. and Portegies Zwart, S. F., "The gravitational wave signal from the Galactic disk population of binaries containing two compact objects", Astron. Astrophys., 375, 890-898 (2001). [DOI], [ADS], [arXiv:astro-ph/0105221]. (Cited on pages 35, 52, 84, 85, 88, 93, 94, 95, 97, 98, 99, and 105.)

[519] Nelemans, G., Yungelson, L. R. and Portegies Zwart, S. F., "Short-Period AM CVn systems as optical, X-ray and gravitational-wave sources", Mon. Not. R. Astron. Soc., 349, 181-192 (2004). [DOI], [ADS]. (Cited on pages 85, 88, 93, 94, 95, 96, 97, 99, 100, 103, 105, 106, and 107.)

[520] Nelemans, G., Yungelson, L. R., Portegies Zwart, S. F. and Verbunt, F., "Population synthesis for double white dwarfs. I. Close detached systems", Astron. Astrophys., 365, 491-507 (2001). [DOI], [ADS], [arXiv:astro-ph/0010457]. (Cited on pages 41, 72, and 79.)

[521] Nelemans, G., Yungelson, L. R., van der Sluys, M. V. and Tout, C. A., "The chemical composition of donors in AM CVn stars and ultracompact X-ray binaries: observational tests of their formation", Mon. Not. R. Astron. Soc., 401, 1347-1359 (2010). [DOI], [ADS], [arXiv:0909.3376 [astro-ph.SR]]. (Cited on pages $78,83,89$, and 90.)

[522] Nelson, L. A., Rappaport, S. A. and Joss, P. C., "The evolution of ultrashort period binary systems", Astrophys. J., 304, 231-240 (1986). [DOI], [ADS]. (Cited on page 82.)

[523] Neo, S., Miyaji, S., Nomoto, K. and Sugimoto, D., "Effect of Rapid Mass Accretion onto the MainSequence Stars", Publ. Astron. Soc. Japan, 29, 249-262 (1977). [ADS]. (Cited on page 36.)

[524] Ness, J.-U. et al., "Obscuration effects in super-soft-source X-ray spectra", Astron. Astrophys., 559, A50 (2013). [DOI], [ADS], [arXiv:1309.2604 [astro-ph.GA]]. (Cited on page 61.)

[525] Newsham, G., Starrfield, S. and Timmes, F. X., "Evolution of accreting white dwarfs; some of them continue to grow", arXiv, e-print, (2013). [ADS], [arXiv:1303.3642 [astro-ph.SR]]. (Cited on page 69.)

[526] Nice, D. J., Sayer, R. W. and Taylor, J. H., "PSR J1518+4904: A Mildly Relativistic Binary Pulsar System", Astrophys. J. Lett., 466, L87-L90 (1996). [DOI]. (Cited on page 15.)

[527] Nice, D. J., Splaver, E. M. and Stairs, I. H., "Arecibo Measurements of Pulsar-White Dwarf Binaries: Evidence for Heavy Neutron Stars", in Rasio, F. A. and Stairs, I. H., eds., Binary Radio Pulsars, Proceedings of a meeting held at the Aspen Center for Physics, Colorado, USA, 12-16 January 2004, ASP Conference Series, 328, p. 371, (Astronomical Society of the Pacific, San Francisco, 2004). [ADS]. (Cited on page 14.) 
[528] Nielsen, M. T. B., Nelemans, G., Voss, R. and Toonen, S., "On double-degenerate type Ia supernova progenitors as supersoft X-ray sources", Astron. Astrophys., 563, A16 (2014). [DOI], [ADS], [arXiv:1310.2170]. (Cited on page 61.)

[529] Nielsen, M. T. B., Voss, R. and Nelemans, G., "Upper limits on bolometric luminosities of three Type Ia supernova progenitors: new results in the ongoing Chandra archival search for Type Ia supernova progenitors", Mon. Not. R. Astron. Soc., 435, 187-193 (2013). [DOI], [ADS], [arXiv:1308.5821 [astroph.HE]]. (Cited on page 72.)

[530] Nissanke, S., Kasliwal, M. and Georgieva, A., "Identifying elusive electromagnetic counterparts to gravitational wave mergers: An end-to-end simulation", Astrophys. J., 767, 124 (2013). [DOI], [ADS], [arXiv:1210.6362 [astro-ph.HE]]. (Cited on page 56.)

[531] Nissanke, S., Vallisneri, M., Nelemans, G. and Prince, T. A., "Gravitational-wave Emission from Compact Galactic Binaries", Astrophys. J., 758, 131 (2012). [DOI], [ADS], [arXiv:1201.4613 [astroph.GA]]. (Cited on pages 92, 93, 95, 97, 99, 101, and 102.)

[532] Nomoto, K., "Accreting white dwarf models for type I supernovae. I. Presupernova evolution and triggering mechanisms", Astrophys. J., 253, 798-810 (1982). [DOI], [ADS]. (Cited on page 66.)

[533] Nomoto, K. and Iben Jr, I., "Carbon ignition in a rapidly accreting degenerate dwarf: A clue to the nature of the merging process in close binaries", Astrophys. J., 297, 531-537 (1985). [DOI], [ADS]. (Cited on page 73.)

[534] Nomoto, K., Nariai, K. and Sugimoto, D., "Rapid Mass Accretion onto White Dwarfs and Formation of an Extended Envelope", Publ. Astron. Soc. Japan, 31, 287-298 (1979). [ADS]. (Cited on page 37.)

[535] Nomoto, K., Saio, H., Kato, M. and Hachisu, I., "Thermal Stability of White Dwarfs Accreting Hydrogen-rich Matter and Progenitors of Type Ia Supernovae", Astrophys. J., 663, 1269-1276 (2007). [DOI], [ADS], [arXiv:astro-ph/0603351]. (Cited on pages 60 and 61.)

[536] Nordhaus, J., Brandt, T. D., Burrows, A. and Almgren, A., "The Hydrodynamic Origin of Neutron Star Kicks", Mon. Not. R. Astron. Soc., 423, 1805-1812 (2012). [DOI], [ADS], [arXiv:1112.3342]. (Cited on page 34.)

[537] Noriega-Crespo, A., "Star Formation in the Milky Way: The Infrared View", in Torres, D. F. and Reimer, O., eds., Cosmic Rays in Star-Forming Environments, Astrophysics and Space Science Proceedings, 34, pp. 29-40, (Springer, Berlin; New York, 2013). [DOI], [ADS], [arXiv:1302.1196 [astro-ph.GA]]. (Cited on pages 9 and 17.)

[538] Noutsos, A., Schnitzeler, D. H. F. M., Keane, E. F., Kramer, M. and Johnston, S., "Pulsar spinvelocity alignment: kinematic ages, birth periods and braking indices", Mon. Not. R. Astron. Soc., 430, 2281-2301 (2013). [DOI], [ADS], [arXiv:1301.1265]. (Cited on page 34.)

[539] Nugent, P. E. et al., "Supernova SN 2011fe from an exploding carbon-oxygen white dwarf star", Nature, 480, 344-347 (2011). [DOI], [ADS], [arXiv:1110.6201 [astro-ph.CO]]. (Cited on page 65.)

[540] Nugis, T. and Lamers, H. J. G. L. M., "Mass-loss rates of Wolf-Rayet stars as a function of stellar parameters", Astron. Astrophys., 360, 227-244 (2000). [ADS]. (Cited on page 44.)

[541] Oliveira, A. S. and Steiner, J. E., "WX Cen (三WR 48c) - a possible Type Ia supernova progenitor", Mon. Not. R. Astron. Soc., 351, 685-693 (2004). [DOI], [ADS], [arXiv:astro-ph/0403281]. (Cited on page 68.)

[542] Orosz, J. A., "Inventory of black hole binaries", in van der Hucht, K. A., Herrero, A. and Esteban, C., eds., A Massive Star Odyssey: From Main Sequence to Supernova, Conference in Lanzarote, Spain, 24-28 June 2002, IAU Symposia, 212, p. 365, (Astronomical Society of the Pacific, San Francisco, 2003). [astro-ph/0209041]. (Cited on page 48.) 
[543] Orosz, J. A., McClintock, J. E., Aufdenberg, J. P., Remillard, R. A., Reid, M. J., Narayan, R. and Gou, L., "The Mass of the Black Hole in Cygnus X-1", Astrophys. J., 742, 84 (2011). [DOI], [ADS], [arXiv:1106.3689 [astro-ph.HE]]. (Cited on page 19.)

[544] Orosz, J. A. et al., "A 15.65-solar-mass black hole in an eclipsing binary in the nearby spiral galaxy M 33", Nature, 449, 872-875 (2007). [DOI], [ADS], [arXiv:0710.3165]. (Cited on page 19.)

[545] Orosz, J. A. et al., "A New Dynamical Model for the Black Hole Binary LMC X-1", Astrophys. J., 697, 573-591 (2009). [DOI], [ADS], [arXiv:0810.3447]. (Cited on page 19.)

[546] Osaki, Y., "An accretion model for the outbursts of U Geminorum stars", Publ. Astron. Soc. Japan, 26, 429-436 (1974). [ADS]. (Cited on page 60.)

[547] O’Shaughnessy, R., Kim, C., Fragos, T., Kalogera, V. and Belczynski, K., "Constraining Population Synthesis Models via the Binary Neutron Star Population", Astrophys. J., 633, 1076-1084 (2005). [DOI], [ADS], [astro-ph/0504479]. (Cited on page 52.)

[548] Osłowski, S., Bulik, T., Gondek-Rosińska, D. and Belczyński, K., "Population synthesis of double neutron stars", Mon. Not. R. Astron. Soc., 413, 461-479 (2011). [DOI], [ADS], [arXiv:0903.3538 [astro-ph.GA]]. (Cited on page 46.)

[549] Özel, F., Psaltis, D., Narayan, R. and McClintock, J. E., "The Black Hole Mass Distribution in the Galaxy", Astrophys. J., 725, 1918-1927 (2010). [DOI], [ADS], [arXiv:1006.2834 [astro-ph.GA]]. (Cited on page 18.)

[550] Özel, F., Psaltis, D., Narayan, R. and Santos Villarreal, A., "On the Mass Distribution and Birth Masses of Neutron Stars", Astrophys. J., 757, 55 (2012). [DOI], [ADS], [arXiv:1201.1006 [astroph.HE]]. (Cited on pages 8 and 18.)

[551] Paczyński, B., "Gravitational Waves and the Evolution of Close Binaries", Acta Astron., 17, 287 (1967). [ADS]. (Cited on pages 57, 79, and 85.)

[552] Paczyński, B., "Evolution of Single Stars. IV. Helium Stars", Acta Astron., 21, 1 (1971). [ADS]. (Cited on pages 75 and 88.)

[553] Paczyński, B., "Common envelope binaries", in Eggleton, P. P., Mitton, S. and Whelan, J., eds., Structure and Evolution of Close Binary Systems, Conference in Cambridge, U.K., 28 July - 1 August 1975, IAU Symposium, 73, pp. 75-80, (D. Reidel, Dordrecht; Boston, 1976). [ADS]. (Cited on pages 38 and 79.)

[554] Paczyński, B., "A model of accretion disks in close binaries", Astrophys. J., 216, 822-826 (1977). [DOI], [ADS]. (Cited on page 62.)

[555] Paczyński, B. and Sienkiewicz, R., "Evolution of Close Binaries VIII. Mass Exchange on the Dynamical Time Scale", Acta Astron., 22, 73-91 (1972). [ADS]. (Cited on pages 37, 58, and 67.)

[556] Paczyński, B. and Ziółkowski, J., "Evolution of Close Binaries. III.", Acta Astron., 17, 7-14 (1967). [ADS]. (Cited on page 28.)

[557] Paczyński, B. and Ziółkowski, J., "On the Origin of Planetary Nebulae and Mira Variables", Acta Astron., 18, 255 (1968). [ADS]. (Cited on page 9.)

[558] Paczyński, B., Ziólkowski, J. and Zytkow, A., "On the Time-Scale of the Mass Transfer in Close Binaries", in Hack, M., ed., Mass Loss from Stars, Proceedings of the second Trieste Colloquium on Astrophysics, 12 -17 September, 1968, Astrophysics and Space Science Library, 13, p. 237, (Kluwer, Dordrecht, 1969). [ADS]. (Cited on page 37.)

[559] Paczyński, B. and Zytkow, A. N., "Hydrogen shell flashes in a white dwarf with mass accretion", Astrophys. J., 222, 604-611 (1978). [DOI], [ADS]. (Cited on pages 37 and 60.) 
[560] Pakmor, R., Kromer, M., Taubenberger, S. and Springel, V., "Helium-ignited Violent Mergers as a Unified Model for Normal and Rapidly Declining Type Ia Supernovae", Astrophys. J. Lett., 770, L8 (2013). [DOI], [ADS], [arXiv:1302.2913 [astro-ph.HE]]. (Cited on pages 76 and 77.)

[561] Palacios, A., "Influence of Rotation on Stellar Evolution", in Hennebelle, P. and Charbonnel, C., eds., Angular Momentum Transport During Star Formation and Evolution, Evry Schatzman School on Stellar Physics (EES 2012), Aussois, France, September 2012, EAS Publications Series, 62, pp. 227-287, (EDP Sciences, Les Ulis, 2013). [DOI], [ADS], [arXiv:1307.1316]. (Cited on page 11.)

[562] Palenzuela, C., Lehner, L., Liebling, S. L., Ponce, M., Anderson, M., Neilsen, D. and Motl, P., "Linking electromagnetic and gravitational radiation in coalescing binary neutron stars", Phys. Rev. $D, \mathbf{8 8}, 043011$ (2013). [DOI], [ADS], [arXiv:1307.7372 [gr-qc]]. (Cited on page 56.)

[563] Pan, K.-C., Ricker, P. M. and Taam, R. E., "Impact of Type Ia Supernova Ejecta on Binary Companions in the Single-degenerate Scenario", Astrophys. J., 750, 151 (2012). [DOI], [ADS], [arXiv:1203.1932 [astro-ph.HE]]. (Cited on page 69.)

[564] Pan, T. and Loeb, A., "Identifying stars of mass $>150 M_{\odot}$ from their eclipse by a binary companion", Mon. Not. R. Astron. Soc., 425, L91-L95 (2012). [DOI], [ADS], [arXiv:1206.1050 [astro-ph.SR]]. (Cited on page 8.)

[565] Panagia, N., Van Dyk, S. D., Weiler, K. W., Sramek, R. A., Stockdale, C. J. and Murata, K. P., "A Search for Radio Emission from Type Ia Supernovae", Astrophys. J., 646, 369-377 (2006). [DOI], [ADS], [arXiv:astro-ph/0603808]. (Cited on page 69.)

[566] Pandey, G., Lambert, D. L., Jeffery, C. S. and Rao, N. K., "An Analysis of Ultraviolet Spectra of Extreme Helium Stars and New Clues to Their Origins", Astrophys. J., 638, 454-471 (2006). [DOI], [ADS]. (Cited on page 76.)

[567] Papaloizou, J. and Pringle, J. E., "Tidal torques on accretion discs in close binary systems", Mon. Not. R. Astron. Soc., 181, 441-454 (1977). [ADS]. (Cited on page 62.)

[568] Papish, O., Nordhaus, J. and Soker, N., "Problems with neutrino-driven core-collapse supernova mechanisms", arXiv, e-print, (2014). [ADS], [arXiv:1402.4362 [astro-ph.SR]]. (Cited on page 48.)

[569] Parker, R. J. and Reggiani, M. M., "The binary companion mass ratio distribution: an imprint of the star formation process?", Mon. Not. R. Astron. Soc., 432, 2378-2384 (2013). [DOI], [ADS], [arXiv:1304.3123 [astro-ph.GA]]. (Cited on page 12.)

[570] Passy, J.-C., Herwig, F. and Paxton, B., "The Response of Giant Stars to Dynamical-timescale Mass Loss", Astrophys. J., 760, 90 (2012). [DOI], [ADS], [arXiv:1111.4202 [astro-ph.SR]]. (Cited on page 41.)

[571] Passy, J.-C. et al., "Simulating the Common Envelope Phase of a Red Giant Using Smoothedparticle Hydrodynamics and Uniform-grid Codes", Astrophys. J., 744, 52 (2012). [DOI], [ADS], [arXiv:1107.5072 [astro-ph.SR]]. (Cited on pages 39 and 62.)

[572] Patat, F. et al., "Detection of Circumstellar Material in a Normal Type Ia Supernova", Science, 317, 924 (2007). [DOI], [ADS], [arXiv:0707.2793]. (Cited on page 70.)

[573] Pauli, E.-M., Napiwotzki, R., Heber, U., Altmann, M. and Odenkirchen, M., "3D kinematics of white dwarfs from the SPY project. II.", Astron. Astrophys., 447, 173-184 (2006). [DOI], [ADS], [astro-ph/0510494]. (Cited on page 79.)

[574] Paxton, B., Bildsten, L., Dotter, A., Herwig, F., Lesaffre, P. and Timmes, F., "Modules for Experiments in Stellar Astrophysics (MESA)", Astrophys. J. Suppl. Ser., 192, 3 (2011). [DOI], [ADS], [arXiv:1009.1622 [astro-ph.SR]]. (Cited on page 58.) 
[575] Paxton, B. et al., "Modules for Experiments in Stellar Astrophysics (MESA): Giant Planets, Oscillations, Rotation, and Massive Stars", Astrophys. J. Suppl. Ser., 208, 4 (2013). [DOI], [ADS], [arXiv:1301.0319 [astro-ph.SR]]. (Cited on page 58.)

[576] Perets, H. B. et al., "A faint type of supernova from a white dwarf with a helium-rich companion", Nature, 465, 322-325 (2010). [DOI], [ADS], [arXiv:0906.2003 [astro-ph.HE]]. (Cited on page 83.)

[577] Pérez-González, P. G., Zamorano, J., Gallego, J., Aragón-Salamanca, A. and Gil de Paz, A., "Spatial Analysis of the H $\alpha$ Emission in the Local Star-Forming UCM Galaxies", Astrophys. J., 591, 827-842 (2003). [DOI]. (Cited on page 17.)

[578] Perlmutter, S. et al., "Measurements of Omega and Lambda from 42 High-Redshift Supernovae", Astrophys. J., 517, 565-586 (1999). [DOI], [ADS]. (Cited on page 7.)

[579] Peters, P. C., "Gravitational Radiation and the Motion of Two Point Masses", Phys. Rev., 136, B1224-B1232 (1964). [DOI], [ADS]. (Cited on pages 14, 24, and 25.)

[580] Petrov, V. S., Cherepashchuk, A. M. and Antokhina, E. A., "Stability of the parameters of the Stellar black-hole mass distribution estimated using nonparametric methods", Astron. Rep., 58, 113-125 (2014). [DOI], [ADS]. (Cited on page 18.)

[581] Pfahl, E., Podsiadlowski, P. and Rappaport, S., "Relativistic Binary Pulsars with Black Hole Companions", Astrophys. J., 628, 343-352 (2005). [DOI], [ADS]. (Cited on page 109.)

[582] Pfahl, E., Rappaport, S. and Podsiadlowski, P., "A Comprehensive Study of Neutron Star Retention in Globular Clusters", Astrophys. J., 573, 283-305 (2002). [DOI], [ADS]. (Cited on page 34.)

[583] Pfahl, E., Rappaport, S., Podsiadlowski, P. and Spruit, H., "A New Class of High-Mass X-Ray Binaries: Implications for Core Collapse and Neutron Star Recoil", Astrophys. J., 574, 364-376 (2002). [DOI], [ADS]. (Cited on pages 34, 35, and 42.)

[584] Phillips, M. M., "The absolute magnitudes of Type Ia supernovae”, Astrophys. J. Lett., 413, L105L108 (1993). [DOI], [ADS]. (Cited on page 77.)

[585] Philp, C. J., Evans, C. R., Leonard, P. J. T. and Frail, D. A., "DO OB Runaway Stars Have Pulsar Companions?", Astron. J., 111, 1220 (1996). [DOI], [ADS], [arXiv:astro-ph/9509087]. (Cited on page 44.)

[586] Phinney, E. S., "The rate of neutron star binary mergers in the universe: Minimal predictions for gravity wave detectors", Astrophys. J. Lett., 380, L17-L21 (1991). [DOI]. (Cited on pages 8 and 16.)

[587] Piersanti, L., Cassisi, S., Iben Jr, I. and Tornambé, A., "On the Very Long Term Evolutionary Behavior of Hydrogen-accreting Low-Mass CO White Dwarfs", Astrophys. J. Lett., 521, L59-L62 (1999). [DOI]. (Cited on page 60.)

[588] Piersanti, L., Cassisi, S., Iben Jr, I. and Tornambé, A., "Hydrogen-Accreting Carbon-Oxygen White Dwarfs of Low Mass: Thermal and Chemical Behavior of Burning Shells", Astrophys. J., 535, 932942 (2000). [DOI]. (Cited on page 60.)

[589] Piersanti, L., Gagliardi, S., Iben Jr, I. and Tornambé, A., "Carbon-Oxygen White Dwarf Accreting CO-Rich Matter. II. Self-Regulating Accretion Process up to the Explosive Stage", Astrophys. J., 598, 1229-1238 (2003). [DOI], [ADS]. (Cited on pages 73 and 76.)

[590] Piersanti, L., Tornambé, A., Yungelson, L. R. and Straniero, O., "He-accreting WDs as SNe Ia Progenitors", in Di Stefano, R., Orio, M. and Moe, M., eds., Binary Paths to Type Ia Supernovae Explosions, Proceedings of IAU Symposium 281, July 2011, Proc. IAU, 281, pp. 209-212, (Cambridge University Press, Cambridge, 2013). [DOI], [ADS]. (Cited on pages 36, 66, and 68.)

[591] Piro, A. L., "The Internal Shear of Type Ia Supernova Progenitors During Accretion and Simmering", Astrophys. J., 679, 616-625 (2008). [DOI], [ADS], [arXiv:0801.1107]. (Cited on page 69.) 
[592] Piro, A. L. and Kulkarni, S. R., "Radio Transients from the Accretion-induced Collapse of White Dwarfs", Astrophys. J. Lett., 762, L17 (2013). [DOI], [ADS], [arXiv:1211.0547 [astro-ph.HE]]. (Cited on page 34.)

[593] Piro, A. L. and Nakar, E., "What can we Learn from the Rising Light Curves of Radioactively Powered Supernovae?", Astrophys. J., 769, 67 (2013). [DOI], [ADS], [arXiv:1210.3032 [astro-ph.HE]]. (Cited on page 65.)

[594] Plavec, M., Ulrich, R. K. and Polidan, R. S., "Mass Loss from Convective Envelopes of Giant Components of Close Binary Systems", Publ. Astron. Soc. Pac., 85, 769 (1973). [DOI], [ADS]. (Cited on page 58.)

[595] Podsiadlowski, P., Dewi, J. D. M., Lesaffre, P., Miller, J. C., Newton, W. G. and Stone, J. R., "The Double Pulsar J0737-3039: Testing the Neutron Star Equation of State", Mon. Not. R. Astron. Soc., 361, 1243-1249 (2005). [DOI], [astro-ph/0506566]. (Cited on page 34.)

[596] Podsiadlowski, P., Han, Z. and Rappaport, S., "Cataclysmic variables with evolved secondaries and the progenitors of AM CVn stars", Mon. Not. R. Astron. Soc., 340, 1214-1228 (2003). [DOI], [ADS]. (Cited on page 82.)

[597] Podsiadlowski, P., Langer, N., Poelarends, A. J. T., Rappaport, S., Heger, A. and Pfahl, E., "The Effects of Binary Evolution on the Dynamics of Core Collapse and Neutron Star Kicks", Astrophys. J., 612, 1044-1051 (2004). [DOI]. (Cited on pages 10 and 34.)

[598] Podsiadlowski, P., Rappaport, S. and Han, Z., "On the formation and evolution of black hole binaries", Mon. Not. R. Astron. Soc., 341, 385-404 (2003). [DOI]. (Cited on page 52.)

[599] Poelarends, A. J. T., Herwig, F., Langer, N. and Heger, A., "The Supernova Channel of Super-AGB Stars", Astrophys. J., 675, 614-625 (2008). [DOI], [ADS], [arXiv:0705.4643]. (Cited on page 9.)

[600] Politano, M. and Weiler, K. P., "The Distribution of Secondary Masses in Post-Common-Envelope Binaries: A Potential Test of Disrupted Magnetic Braking", Astrophys. J. Lett., 641, L137-L140 (2006). [DOI], [ADS]. (Cited on page 26.)

[601] Pols, O. R., Schröder, K.-P., Hurley, J. R., Tout, C. A. and Eggleton, P. P., "Stellar evolution models for $Z=0.0001$ to 0.03", Mon. Not. R. Astron. Soc., 298, 525-536 (1998). [DOI], [ADS]. (Cited on page 39.)

[602] Popov, S. B. and Prokhorov, M. E., "Population synthesis in astrophysics", Phys. Usp., 50, 11231146 (2007). [DOI], [ADS], [arXiv:astro-ph/0411792]. (Cited on page 52.)

[603] Popova, E. I., Tutukov, A. V. and Yungelson, L. R., "The Influence of Rapid Accretion on the Evolution of Massive Stars", Nauchn. Inform., 42, 45 (1978). [ADS]. (Cited on page 28.)

[604] Popova, E. I., Tutukov, A. V. and Yungelson, L. R., "Study of physical properties of spectroscopic binary stars", Astrophys. Space Sci., 88, 55-80 (1982). [DOI]. (Cited on pages 12 and 50.)

[605] Portegies Zwart, S. F., Makino, J., McMillan, S. L. W. and Hut, P., "Star cluster ecology. III. Runaway collisions in young compact star clusters", Astron. Astrophys., 348, 117-126 (1999). [ADS], [arXiv:astro-ph/9812006]. (Cited on page 8.)

[606] Portegies Zwart, S. F., Verbunt, F. and Ergma, E., "The formation of black-holes in low-mass X-ray binaries", Astron. Astrophys., 321, 207-212 (1997). (Cited on page 48.)

[607] Portegies Zwart, S. F. and Yungelson, L. R., "Formation and Evolution of Binary Neutron Stars", Astron. Astrophys., 332, 173-188 (1998). (Cited on pages 35, 44, 46, and 52.)

[608] Postnov, K. A. and Prokhorov, M. E., "Galactic binary gravitational wave noise within LISA frequency band", Astrophys. J., 494, 674-679 (1998). [DOI], [ADS], [astro-ph/9801034]. (Cited on page 93.) 
[609] Postnov, K. A. and Prokhorov, M. E., "Binary black hole formaiton and merging", in Trân Than Vân, J., Dumarchez, J., Raynoud, S., Salomon, C., Thorsett, S. and Vinet, J. Y., eds., Gravitational Waves and Experimental Gravity, XXXIVth Rencontres de Moriond, Les Arcs, France, 23-30 January 1999, pp. 113-118, (World Publishers, Hanoi, 2000). (Cited on pages 35 and 49.)

[610] Postnov, K. A. and Prokhorov, M. E., "The Relation between the Observed Mass Distribution for Compact Stars and the Mechanism for Supernova Explosions", Astron. Rep., 45, 899-907 (2001). [DOI], [ADS], [astro-ph/0110176]. (Cited on page 18.)

[611] Prestwich, A. H. et al., "The Orbital Period of the Wolf-Rayet Binary IC 10 X-1: Dynamic Evidence that the Compact Object Is a Black Hole", Astrophys. J. Lett., 669, L21-L24 (2007). [DOI], [ADS], [arXiv:0709.2892]. (Cited on page 19.)

[612] Prialnik, D., An Introduction to the Theory of Stellar Structure and Evolution, (Cambridge University Press, Cambridge; New York, 2000). [ADS], [Google Books]. (Cited on page 11.)

[613] Prialnik, D. and Kovetz, A., "An extended grid of multicycle nova evolution models", Astrophys. J., 445, 789-810 (1995). [DOI], [ADS]. (Cited on pages 60, 67, and 68.)

[614] Prialnik, D. and Livio, M., "The outcome of accretion on to a fully convective star. Expansion or contraction?", Mon. Not. R. Astron. Soc., 216, 37-52 (1985). [ADS]. (Cited on page 36.)

[615] Prince, T. A., Anderson, S. B., Kulkarni, S. R. and Wolszczan, A., "Timing observations of the 8 hour binary pulsar 2127+11C in the globular cluster M15", Astrophys. J. Lett., 374, L41-L44 (1991). [DOI]. (Cited on page 15.)

[616] Pringle, J. E., "Soft X-ray emission from dwarf novae", Mon. Not. R. Astron. Soc., 178, 195-202 (1977). [ADS]. (Cited on page 106.)

[617] Prokhorov, M. E. and Postnov, K. A., "Direct observation of the kick during the birth of a neutron star in the binary pulsar PSR B1259-63", Astron. Lett., 23, 439-444 (1997). (Cited on page 34.)

[618] Psaltis, D., "Probes and Tests of Strong-Field Gravity with Observations in the Electromagnetic Spectrum", Living Rev. Relativity, 11, lrr-2008-9 (2008). [DOI], [ADS], [arXiv:0806.1531]. URL (accessed 4 March 2014):

http://www.livingreviews.org/lrr-2008-9. (Cited on page 17.)

[619] Pylyser, E. and Savonije, G. J., "Evolution of low-mass close binary sytems with a compact mass accreting component", Astron. Astrophys., 191, 57-70 (1988). [ADS]. (Cited on page 18.)

[620] Qian, S.-B., Shi, G., Fernández Lajús, E., Di Sisto, R. P., Zhu, L.-Y., Liu, L., Zhao, E.-G. and Li, L.-J., "Is WX Cen a Possible Type Ia Supernova Progenitor with Wind-driven Mass Transfer?", Astrophys. J. Lett., 772, L18 (2013). [DOI], [ADS]. (Cited on page 68.)

[621] Raghavan, D. et al., "A Survey of Stellar Families: Multiplicity of Solar-type Stars", Astrophys. J. Suppl. Ser., 190, 1-42 (2010). [DOI], [ADS], [arXiv:1007.0414 [astro-ph.SR]]. (Cited on page 12.)

[622] Raguzova, N. V. and Popov, S. B., "Be X-ray binaries and candidates", Astron. Astrophys. Trans., 24, 151-185 (2005). [DOI], [ADS]. (Cited on page 45.)

[623] Ramsay, G., Barclay, T., Steeghs, D., Wheatley, P. J., Hakala, P., Kotko, I. and Rosen, S., "The long-term optical behaviour of helium-accreting AM CVn binaries", Mon. Not. R. Astron. Soc., 419, 2836-2843 (2012). [DOI], [ADS], [arXiv:1109.6518 [astro-ph.GA]]. (Cited on pages 85 and 106.)

[624] Ramsay, G., Groot, P. J., Marsh, T. R., Nelemans, G., Steeghs, D. and Hakala, P., "XMM-Newton observations of AM CVn binaries: V396 Hya and SDSS J1240-01", Astron. Astrophys., 457, 623-627 (2006). [DOI], [ADS]. (Cited on page 85.)

[625] Rappaport, S., Di Stefano, R. and Smith, J. D., "Formation and evolution of luminous supersoft X-ray sources", Astrophys. J., 426, 692-703 (1994). [DOI], [ADS]. (Cited on page 61.) 
[626] Rappaport, S. and Joss, P. C., "The lower main sequence and the nature of secondary stars in ultracompact binaries", Astrophys. J., 283, 232-240 (1984). [DOI], [ADS]. (Cited on pages 79 and 85.)

[627] Rappaport, S., Verbunt, F. and Joss, P. C., "A new technique for calculations of binary stellar evolution, with applicationto magnetic braking", Astrophys. J., 275, 713-731 (1983). [DOI], [ADS]. (Cited on page 26.)

[628] Raskin, C., Kasen, D., Moll, R., Schwab, J. and Woosley, S. E., "Type Ia Supernovae from Merging White Dwarfs II) Post-Merger Detonations", arXiv, e-print, (2013). [arXiv:1312.3649 [astro-ph.HE]]. (Cited on page 77.)

[629] Raskin, C., Scannapieco, E., Fryer, C. L., Rockefeller, G. and Timmes, F. X., "Remnants of Binary White Dwarf Mergers", Astrophys. J., 746, 62 (2012). [DOI], [ADS], [arXiv:1112.1420 [astro-ph.HE]]. (Cited on page 77.)

[630] Raskin, C., Scannapieco, E., Rockefeller, G., Fryer, C. L., Diehl, S. and Timmes, F. X., “56 Ni Production in Double-degenerate White Dwarf Collisions", Astrophys. J., 724, 111-125 (2010). [DOI], [ADS], [arXiv:1009.2507 [astro-ph.SR]]. (Cited on page 77.)

[631] Refsdal, S., Roth, M. L. and Weigert, A., "On the binary system AS Eri", Astron. Astrophys., 36, 113-122 (1974). [ADS]. (Cited on pages 28, 36, and 39.)

[632] Reggiani, M. and Meyer, M. R., "Universality of the companion mass-ratio distribution", Astron. Astrophys., 553, A124 (2013). [DOI], [arXiv:1304.3459 [astro-ph.GA]]. (Cited on page 12.)

[633] Remillard, R. A. and McClintock, J. E., "X-Ray Properties of Black-Hole Binaries", Annu. Rev. Astron. Astrophys., 44, 49-92 (2006). [DOI], [ADS], [arXiv:astro-ph/0606352]. (Cited on pages 17, 18, and 48.)

[634] Repetto, S., Davies, M. B. and Sigurdsson, S., "Investigating stellar-mass black hole kicks", Mon. Not. R. Astron. Soc., 425, 2799-2809 (2012). [DOI], [ADS], [arXiv:1203.3077 [astro-ph.GA]]. (Cited on page 35.$)$

[635] Ricci, F. and Brillet, A., "A Review of Gravitational Wave Detectors", Annu. Rev. Nucl. Part. Sci., 47, 111-156 (1997). [DOI], [ADS]. (Cited on page 7.)

[636] Ricker, P. M. and Taam, R. E., "The Interaction of Stellar Objects within a Common Envelope", Astrophys. J. Lett., 672, L41-L44 (2008). [DOI], [ADS], [arXiv:0710.3631]. (Cited on page 39.)

[637] Ricker, P. M. and Taam, R. E., "An AMR Study of the Common-envelope Phase of Binary Evolution", Astrophys. J., 746, 74 (2012). [DOI], [ADS], [arXiv:1107.3889 [astro-ph.SR]]. (Cited on page 39.)

[638] Riess, A. G. et al., "Observational Evidence from Supernovae for an Accelerating Universe and a Cosmological Constant", Astron. J., 116, 1009-1038 (1998). [DOI], [ADS], [arXiv:astro-ph/9805201]. (Cited on page 7.)

[639] Rieutord, M. and Espinosa Lara, F., "Ab Initio Modelling of Steady Rotating Stars", in Goupil, M., Belkacem, K., Neiner, C., Lignières, F. and Green, J. J., eds., Studying Stellar Rotation and Convection, Lecture Notes in Physics, 865, pp. 49-73, (Springer, Berlin; New York, 2013). [DOI], [ADS], [arXiv:1208.4926 [astro-ph.SR]]. (Cited on page 11.)

[640] Rigault, M. et al., "Evidence of environmental dependencies of Type Ia supernovae from the Nearby Supernova Factory indicated by local H $\alpha$ ", Astron. Astrophys., 560, A66 (2013). [DOI], [ADS], [arXiv:1309.1182 [astro-ph.CO]]. (Cited on page 7.)

[641] Rigault, M. et al., "Evidence of Environmental Dependencies of Type Ia Supernovae from the Nearby Supernova Factory indicated by Local H $\alpha$ ", Astron. Astrophys., 560, A66 (2013). [DOI], [ADS], [arXiv:1309.1182 [astro-ph.CO]]. (Cited on pages 7 and 78.) 
[642] Riles, K., "Gravitational waves: Sources, detectors and searches", Prog. Part. Nucl. Phys., 68, 1-54 (2013). [DOI], [ADS], [arXiv:1209.0667 [hep-ex]]. (Cited on page 7.)

[643] Ritchie, B. W., Clark, J. S., Negueruela, I. and Langer, N., "A VLT/FLAMES survey for massive binaries in Westerlund 1. II. Dynamical constraints on magnetar progenitor masses from the eclipsing binary W13", Astron. Astrophys., 520, A48 (2010). [DOI], [ADS], [arXiv:1008.2840 [astro-ph.SR]]. (Cited on pages 12 and 29.)

[644] Ritossa, C., García-Berro, E. and Iben Jr, I., "On the Evolution of Stars that Form Electrondegenerate Cores Processed by Carbon Burning. V. Shell Convection Sustained by Helium Burning, Transient Neon Burning, Dredge-Out, URCA Cooling, and Other Properties of an $11 M_{\odot}$ Population I Model Star", Astrophys. J., 515, 381-397 (1999). [DOI], [ADS]. (Cited on page 34.)

[645] Ritter, H. and Kolb, U., "Catalogue of cataclysmic binaries, low-mass X-ray binaries and related objects (Seventh edition)", Astron. Astrophys., 404, 301-303 (2003). [DOI], [ADS]. (Cited on page 79.)

[646] Ritter, H. and Kolb, U., "Catalogue of Cataclysmic Binaries, Low-Mass X-Ray Binaries and Related Objects", project homepage, The Open University, (2013). URL (accessed 22 April 2014): http://physics.open.ac.uk/RKcat/. (Cited on page 79.)

[647] Robinson, E. L., Barker, E. S., Cochran, A. L., Cochran, W. D. and Nather, R. E., "MV Lyrae: Spectrophotometric properties of minimum light; or on MV Lyrae off", Astrophys. J., 251, 611-619 (1981). [DOI], [ADS]. (Cited on page 25.)

[648] Robinson, E. L. and Shafter, A. W., "An Upper Limit to the Space Density of Short-period Noninteracting Binary White Dwarfs", Astrophys. J., 322, 296 (1987). [DOI], [ADS]. (Cited on page 79.)

[649] Rodriguez, C.L, Farr, B., Raymond, V., Farr, W.M, Littenberg, T. B., Fazi, D. and Kalogera, V., "Basic Parameter Estimation of Binary Neutron Star Systems by the Advanced LIGO/Virgo Network", Astrophys. J., 784, 119 (2014). [DOI], [ADS], [arXiv:1309.3273 [astro-ph.HE]]. (Cited on page 55.)

[650] Rodríguez-Gil, P. et al., "The orbital period of V458 Vulpeculae, a post-double common-envelope nova", Mon. Not. R. Astron. Soc., 407, L21-L25 (2010). [DOI], [ADS]. (Cited on page 80.)

[651] Roelofs, G. H. A., Groot, P. J., Nelemans, G., Marsh, T. R. and Steeghs, D., "On the orbital periods of the AM CVn stars HP Librae and V803 Centauri", Mon. Not. R. Astron. Soc., 379, 176-182 (2007). [DOI], [ADS]. (Cited on page 88.)

[652] Roelofs, G. H. A., Rau, A., Marsh, T. R., Steeghs, D., Groot, P. J. and Nelemans, G., "Spectroscopic Evidence for a 5.4 Minute Orbital Period in HM Cancri", Astrophys. J. Lett., 711, L138-L142 (2010). [DOI], [ADS], [arXiv:1003.0658 [astro-ph.SR]]. (Cited on page 88.)

[653] Rogan, A. and Bose, S., "Parameter estimation of binary compact objects with LISA: Effects of time-delay interferometry, Doppler modulation, and frequency evolution", arXiv, e-print, (2006). [ADS], [arXiv:astro-ph/0605034]. (Cited on page 108.)

[654] Romani, R. W., "A unified model of neutron-star magnetic fields", Nature, 347, 741-743 (1990). [DOI]. (Cited on page 45.)

[655] Romani, R. W., "Neutron Star Magnetic Fields", in Fruchter, A. S., Tavani, M. and Backer, D. C., eds., Millisecond Pulsars: A Decade of Surprise, Conference in Aspen, Colorado, 3 - 7 January 1994, ASP Conference Series, 72, p. 288, (Astronomical Society of the Pacific, San Francisco, 1995). (Cited on page 45.)

[656] Rose, W. K., "A Model for the Nova Outburst", Astrophys. J., 152, 245 (1968). [DOI], [ADS]. (Cited on page 60.) 
[657] Rosenbush, A. E., "List of Helium Novae", in Werner, K. and Rauch, T., eds., Hydrogen-Deficient Stars, Proceedings of the conference held 17-21 September, 2007, at Eberhard Karls University, Tübingen, Germany, ASP Conference Series, 391, pp. 271-272, (Astronomical Society of the Pacific, San Francisco, 2008). [ADS]. (Cited on page 91.)

[658] Rosswog, S., "Mergers of Neutron Star-Black Hole Binaries with Small Mass Ratios: Nucleosynthesis, Gamma-Ray Bursts, and Electromagnetic Transients", Astrophys. J., 634, 1202-1213 (2005). [DOI], [ADS], [arXiv:astro-ph/0508138]. (Cited on page 56.)

[659] Ruderman, M. A. and Shaham, J., "Fate of very low-mass secondaries in accreting binaries and the 1.5-ms pulsar", Nature, 304, 425-427 (1983). [DOI]. (Cited on page 61.)

[660] Ruiter, A. J., Belczynski, K., Benacquista, M. J. and Holley-Bockelmann, K., "The Contribution of Halo White Dwarf Binaries to the Laser Interferometer Space Antenna Signal", Astrophys. J., 693, 383-387 (2009). [DOI], [ADS]. (Cited on page 95.)

[661] Ruiter, A. J., Belczynski, K., Benacquista, M. J., Larson, S. L. and Williams, G., "The LISA Gravitational Wave Foreground: A Study of Double White Dwarfs", Astrophys. J., 717, 1006-1021 (2010). [DOI], [ADS], [arXiv:0705.3272]. (Cited on pages 85, 93, 95, and 97.)

[662] Ruiter, A. J., Belczynski, K. and Fryer, C. L., "Rates and Delay Times of Type Ia Supernovae", Astrophys. J., 699, 2026-2036 (2009). [DOI], [ADS], [arXiv:0904.3108 [astro-ph.SR]]. (Cited on pages 83 and 91.)

[663] Ruiz-Lapuente, P. and Canal, R., "Type Ia Supernova Counts at High z: Signatures of Cosmological Models and Progenitors", Astrophys. J. Lett., 497, L57-L60 (1998). [DOI], [ADS]. (Cited on page 7.)

[664] Sackett, P. D., "Does the Milky Way Have a Maximal Disk?", Astrophys. J., 483, 103 (1997). [DOI], [ADS]. (Cited on page 96.)

[665] Saffer, R. A., Liebert, J. and Olszewski, E. W., "Discovery of a close detached binary DA white dwarf system", Astrophys. J., 334, 947-957 (1988). [DOI]. (Cited on pages 79 and 92.)

[666] Saffer, R. A., Livio, M. and Yungelson, L. R., "Close Binary White Dwarf Systems: Numerous New Detections and Their Interpretation", Astrophys. J., 502, 394 (1998). [DOI]. (Cited on page 79.)

[667] Saio, H. and Jeffery, C. S., "The evolution of a rapidly accreting helium white dwarf to become a low-luminosity helium star", Mon. Not. R. Astron. Soc., 313, 671-677 (2000). [DOI], [ADS]. (Cited on pages 60,75 , and 81 .)

[668] Saio, H. and Jeffery, C. S., "Merged binary white dwarf evolution: rapidly accreting carbon-oxygen white dwarfs and the progeny of extreme helium stars", Mon. Not. R. Astron. Soc., 333, 121-132 (2002). [DOI], [ADS]. (Cited on pages 60 and 76.)

[669] Salpeter, E. E., "The Luminosity Function and Stellar Evolution", Astrophys. J., 121, 161-167 (1955). [DOI], [ADS]. (Cited on page 9.)

[670] Sana, H., James, G. and Gosset, E., "The massive star binary fraction in young open clusters - III. IC 2944 and the Cen OB2 association", Mon. Not. R. Astron. Soc., 416, 817-831 (2011). [DOI], [ADS], [arXiv:1109.2899 [astro-ph.SR]]. (Cited on page 12.)

[671] Sana, H. et al., "Binary Interaction Dominates the Evolution of Massive Stars", Science, 337, 444 (2012). [DOI], [ADS], [arXiv:1207.6397 [astro-ph.SR]]. (Cited on page 12.)

[672] Sathyaprakash, B. S., "Gravitational waves and astrophysical sources", C. R. Physique, 14, 272-287 (2013). [DOI], [ADS]. (Cited on page 7.) 
[673] Sathyaprakash, B. S. and Schutz, B. F., "Physics, Astrophysics and Cosmology with Gravitational Waves", Living Rev. Relativity, 12, lrr-2009-2 (2009). [DOI], [ADS], [arXiv:0903.0338 [gr-qc]]. URL (accessed 4 March 2014):

http://www.livingreviews.org/lrr-2009-2. (Cited on pages 7, 17, and 54.)

[674] Savonije, G. J., de Kool, M. and van den Heuvel, E. P. J., "The minimum orbital period for ultracompact binaries with helium burning secondaries", Astron. Astrophys., 155, 51-57 (1986). [ADS]. (Cited on pages 64 and 88.)

[675] Sayer, R. W., Nice, D. J. and Kaspi, V. M., "A Search for Pulsar Companions to OB Runaway Stars", Astrophys. J., 461, 357 (1996). [DOI], [ADS]. (Cited on page 44.)

[676] Scalzo, R. et al., "Nearby Supernova Factory Observations of SN 2007if: First Total Mass Measurement of a Super-Chandrasekhar-Mass Progenitor", Astrophys. J., 713, 1073-1094 (2010). [DOI], [ADS]. (Cited on page 73.)

[677] Scalzo, R. et al., "A Search for New Candidate Super-Chandrasekhar-mass Type Ia Supernovae in the Nearby Supernova Factory Data Set", Astrophys. J., 757, 12 (2012). [DOI], [ADS]. (Cited on page 73.)

[678] Scalzo, R. et al., "Type Ia supernova bolometric light curves and ejected mass estimates from the Nearby Supernova Factory", arXiv, e-print, (2014). [ADS], [arXiv:1402.6842 [astro-ph.CO]]. (Cited on page 76.$)$

[679] Schaefer, B. E. and Pagnotta, A., "An absence of ex-companion stars in the type Ia supernova remnant SNR 0509-67.5", Nature, 481, 164-166 (2012). [DOI], [ADS]. (Cited on pages 70 and 72.)

[680] Schatzman, E., "A theory of the role of magnetic activity during star formation", Ann. Astrophys., 25, 18 (1962). [ADS]. (Cited on page 25.)

[681] Schatzman, E., "White dwarfs and type I supernovae", in Gratton, L., ed., Star Evolution, Proceedings of the XXVIIIth Course of the International School of Physics "Enrico Fermi", held August 20 -September 1, 1962 at Varenna, Italy, p. 389, (Academic Press, New York, 1963). [ADS]. (Cited on page 61.)

[682] Schiminovich, D. et al., "The GALEX-VVDS Measurement of the Evolution of the Far-Ultraviolet Luminosity Density and the Cosmic Star Formation Rate", Astrophys. J. Lett., 619, L47-L50 (2005). [DOI]. (Cited on page 17.)

[683] Schnurr, O., Casoli, J., Chené, A.-N., Moffat, A. F. J. and St-Louis, N., "The very massive binary NGC 3603-A1", Mon. Not. R. Astron. Soc., 389, L38-L42 (2008). [DOI], [ADS], [arXiv:0806.2815]. (Cited on page 8.)

[684] Schuerman, D. W., "Roche Potentials Including Radiation Effects", Astrophys. Space Sci., 19, 351358 (1972). [DOI], [ADS]. (Cited on page 26.)

[685] Schwab, J., Podsiadlowski, P. and Rappaport, S., "Further Evidence for the Bimodal Distribution of Neutron-star Masses", Astrophys. J., 719, 722-727 (2010). [DOI], [ADS], [arXiv:1006.4584 [astroph.HE]]. (Cited on pages 42 and 44.)

[686] Seitenzahl, I. R., Cescutti, G., Röpke, F. K., Ruiter, A. J. and Pakmor, R., "Solar abundance of manganese: a case for the existence of near Chandrasekhar-mass Type Ia supernova progenitors", Astron. Astrophys., 559, L5 (2013). [DOI], [ADS], [arXiv:1309.2397 [astro-ph.SR]]. (Cited on page 76.)

[687] Shah, S., Nelemans, G. and van der Sluys, M., "Using electromagnetic observations to aid gravitational-wave parameter estimation of compact binaries observed with LISA. II. The effect of knowing the sky position", Astron. Astrophys., 553, A82 (2013). [DOI], [ADS], [arXiv:1303.6116 [astro-ph.IM]]. (Cited on page 105.) 
[688] Shah, S., van der Sluys, M. and Nelemans, G., "Using electromagnetic observations to aid gravitational-wave parameter estimation of compact binaries observed with LISA", Astron. Astrophys., 544, A153 (2012). [DOI], [ADS], [arXiv:1207.6770 [astro-ph.IM]]. (Cited on page 105.)

[689] Shahbaz, T., Watson, C. A. and Dhillon, V. S., "The spotty donor star in the X-ray transient Cen X4", Mon. Not. R. Astron. Soc., 440, 504-513 (2014). [DOI], [ADS], [arXiv:1402.1639 [astro-ph.SR]]. (Cited on page 26.)

[690] Shakura, N. I. and Sunyaev, R. A., "Black holes in binary systems. Observational appearance", Astron. Astrophys., 24, 337-355 (1973). [ADS]. (Cited on page 17.)

[691] Shapiro, S. L. and Teukolsky, S. A., Black Holes, White Dwarfs, and Neutron Stars: The Physics of Compact Objects, (Wiley, New York, 1983). [Google Books]. (Cited on page 11.)

[692] Shappee, B. J., Kochanek, C. S. and Stanek, K. Z., "Type Ia single degenerate survivors must be overluminous", Astrophys. J., 765, 150 (2013). [DOI], [ADS], [arXiv:1205.5028 [astro-ph.SR]]. (Cited on page 69.)

[693] Shappee, B. J. and Thompson, T. A., "The Mass-loss-induced Eccentric Kozai Mechanism: A New Channel for the Production of Close Compact Object-Stellar Binaries", Astrophys. J., 766, 64 (2013). [DOI], [ADS], [arXiv:1204.1053 [astro-ph.SR]]. (Cited on pages 21 and 37.)

[694] Shara, M. M., Prialnik, D. and Kovetz, A., "Multiple hydrogen flashes on accreting low-mass white dwarfs - Novae and DAO stars", Astrophys. J., 406, 220-228 (1993). [DOI], [ADS]. (Cited on page 60.)

[695] Shara, M. M., Yaron, O., Prialnik, D., Kovetz, A. and Zurek, D., "An Extended Grid of Nova Models. III. Very Luminous, Red Novae", Astrophys. J., 725, 831-841 (2010). [DOI], [ADS], [arXiv:1009.3864 [astro-ph.SR]]. (Cited on page 60.)

[696] Shen, K. J. and Bildsten, L., "Thermally Stable Nuclear Burning on Accreting White Dwarfs", Astrophys. J., 660, 1444-1450 (2007). [DOI], [ADS], [arXiv:astro-ph/0702049]. (Cited on page 60.)

[697] Shen, K. J. and Bildsten, L., "Unstable Helium Shell Burning on Accreting White Dwarfs", Astrophys. J., 699, 1365-1373 (2009). [DOI], [ADS], [arXiv:0903.0654 [astro-ph.HE]]. (Cited on pages 83 and 91.)

[698] Shen, K. J. and Bildsten, L., "The Ignition of Carbon Detonations via Converging Shock Waves in White Dwarfs", arXiv, e-print, (2013). [ADS], [arXiv:1305.6925 [astro-ph.HE]]. (Cited on page 91.)

[699] Shen, K. J., Bildsten, L., Kasen, D. and Quataert, E., "The Long-term Evolution of Double White Dwarf Mergers", Astrophys. J., 748, 35 (2012). [DOI], [ADS], [arXiv:1108.4036 [astro-ph.HE]]. (Cited on page 73.)

[700] Shen, K. J., Guillochon, J. and Foley, R. J., "Circumstellar Absorption in Double Detonation Type Ia Supernovae", Astrophys. J. Lett., 770, L35 (2013). [DOI], [ADS], [arXiv:1302.2916 [astro-ph.SR]]. (Cited on page 76.)

[701] Shen, K. J., Idan, I. and Bildsten, L., "Helium Core White Dwarfs in Cataclysmic Variables", Astrophys. J., 705, 693-703 (2009). [DOI], [ADS], [arXiv:0906.3767 [astro-ph.HE]]. (Cited on page 60.)

[702] Shen, K. J., Kasen, D., Weinberg, N. N., Bildsten, L. and Scannapieco, E., "Thermonuclear .Ia Supernovae from Helium Shell Detonations: Explosion Models and Observables", Astrophys. J., 715, 767-774 (2010). [DOI], [ADS], [arXiv:1002.2258 [astro-ph.HE]]. (Cited on pages 83 and 91.)

[703] Shiode, J. H., Quataert, E. and Arras, P., "The stability of massive main-sequence stars as a function of metallicity", Mon. Not. R. Astron. Soc., 423, 3397-3404 (2012). [DOI], [ADS], [arXiv:1204.4741 [astro-ph.SR]]. (Cited on page 9.) 
[704] Shklovskii, I. S., "Possible causes of the secular increase in pulsar periods", Sov. Astron., 13, 562-565 (1970). (Cited on page 33.)

[705] Shore, S. N., "Observations and physical processes in binary stars", in Shore, S. N., Livio, M., van den Heuvel, E. P. J., Nussbaumer, H. and Orr, A., eds., Interacting Binaries, Lecture Notes of the Saas-Fee Advanced Course 22, Switzerland, 1992, Saas-Fee Advanced Courses, 22, pp. 1-133, (Springer, Berlin; New York, 1994). [ADS]. (Cited on page 28.)

[706] Siellez, K., Boër, M. and Gendre, B., "Simultaneous event detection rates by electromagnetic and gravitational wave detectors in the advanced era of LIGO and Virgo", Mon. Not. R. Astron. Soc., 437, 649-655 (2014). [DOI], [ADS], [arXiv:1310.2106 [astro-ph.HE]]. (Cited on page 56.)

[707] Siess, L., "Evolution of massive AGB stars. I. Carbon burning phase", Astron. Astrophys., 448, 717-729 (2006). [DOI], [ADS]. (Cited on page 9.)

[708] Siess, L., "Evolution of massive AGB stars. II. model properties at non-solar metallicity and the fate of Super-AGB stars", Astron. Astrophys., 476, 893-909 (2007). [DOI], [ADS]. (Cited on page 9.)

[709] Siess, L., "The most massive AGB stars", in Deng, L. and Chan, K. L., eds., The Art of Modeling Stars in the 21st Century, Proceedings of IAU Symposium 252, April 2008, Proc. IAU, 252, pp. 297-307, (Cambridge University Press, Cambridge, 2008). [DOI], [ADS]. (Cited on page 10.)

[710] Silverman, J. M. and Filippenko, A. V., "On IC 10 X-1, the Most Massive Known Stellar-Mass Black Hole", Astrophys. J. Lett., 678, L17-L20 (2008). [DOI], [ADS], [arXiv:0802.2716]. (Cited on page 19.)

[711] Silverman, J. M., Ganeshalingam, M., Li, W., Filippenko, A. V., Miller, A. A. and Poznanski, D., "Fourteen months of observations of the possible super-Chandrasekhar mass Type Ia Supernova 2009dc", Mon. Not. R. Astron. Soc., 410, 585-611 (2011). [DOI], [ADS]. (Cited on page 73.)

[712] Silverman, J. M. et al., "Type Ia Supernovae Strongly Interacting with Their Circumstellar Medium", Astrophys. J. Suppl. Ser., 207, 3 (2013). [DOI], [ADS], [arXiv:1304.0763 [astro-ph.CO]]. (Cited on page 77.)

[713] Sim, S. A., Fink, M., Kromer, M., Röpke, F. K., Ruiter, A. J. and Hillebrandt, W., "2D simulations of the double-detonation model for thermonuclear transients from low-mass carbon-oxygen white dwarfs", Mon. Not. R. Astron. Soc., 420, 3003-3016 (2012). [DOI], [ADS], [arXiv:1111.2117 [astroph.HE]]. (Cited on pages 74 and 91.)

[714] Sion, E. M., Bond, H. E., Lindler, D., Godon, P., Wickramasinghe, D., Ferrario, L. and Dupuis, J., "Hubble Space Telescope FUV Spectra of the Post-common-envelope Hyades Binary V471 Tauri", Astrophys. J., 751, 66 (2012). [DOI], [ADS], [arXiv:1203.4787 [astro-ph.SR]]. (Cited on page 37.)

[715] Sion, E. M., Holberg, J. B., Oswalt, T. D., McCook, G. P. and Wasatonic, R., "The White Dwarfs Within 20 Parsecs of the Sun: Kinematics and Statistics", Astron. J., 138, 1681-1689 (2009). [DOI], [ADS], [arXiv:0910.1288 [astro-ph.SR]]. (Cited on page 108.)

[716] Sion, E. M., Holberg, J. B., Oswalt, T. D., McCook, G. P., Wasatonic, R. and Myszka, J., "The White Dwarfs within 25 Parsecs of the Sun: Kinematics and Spectroscopic Subtypes", arXiv, e-print, (2014). [ADS], [arXiv:1401.4989 [astro-ph.SR]]. (Cited on page 108.)

[717] Sion, E. M., Linnell, A. P., Godon, P. and Ballouz, R.-L., "The Hot Components of AM CVn Helium Cataclysmics", Astrophys. J., 741, 63 (2011). [DOI], [ADS], [arXiv:1108.1388 [astro-ph.SR]]. (Cited on page 85.)

[718] Skumanich, A., "Time Scales for Ca II Emission Decay, Rotational Braking, and Lithium Depletion", Astrophys. J., 171, 565-567 (1972). [DOI], [ADS]. (Cited on page 25.)

[719] Smak, J., "Light Variability of HZ 29", Acta Astron., 17, 255-270 (1967). [ADS]. (Cited on page 57.) 
[720] Smith, N., "The Crab nebula and the class of Type IIn-P supernovae caused by sub-energetic electroncapture explosions", Mon. Not. R. Astron. Soc., 434, 102-113 (2013). [DOI], [ADS], [arXiv:1304.0689 [astro-ph.HE]]. (Cited on page 44.)

[721] Smith, N., "Mass Loss: Its Effect on the Evolution and Fate of High-Mass Stars", arXiv, e-print, (2014). [ADS], [arXiv:1402.1237 [astro-ph.SR]]. (Cited on page 12.)

[722] Soberman, G. E., Phinney, E. S. and van den Heuvel, E. P. J., "Stability criteria for mass transfer in binary stellar evolution", Astron. Astrophys., 327, 620-635 (1997). (Cited on pages 29 and 37.)

[723] Soker, N., García-Berro, E. and Althaus, L. G., "The explosion of supernova 2011fe in the frame of the core-degenerate scenario", Mon. Not. R. Astron. Soc., 437, L66-L70 (2014). [DOI], [ADS], [arXiv:1309.0368 [astro-ph.SR]]. (Cited on page 77.)

[724] Soker, N. and Harpaz, A., "Criticism of recent calculations of common envelope ejection", Mon. Not. R. Astron. Soc., 343, 456-458 (2003). [DOI], [ADS], [arXiv:astro-ph/0209141]. (Cited on page 39.)

[725] Solheim, J.-E., "AM CVn Stars: Status and Challenges", Publ. Astron. Soc. Pac., 122, 1133-1163 (2010). [DOI], [ADS]. (Cited on pages 83, 84, 85, and 89.)

[726] Solheim, J.-E. and Nasser, M. R., "The AM CVn Systems - The Final Stage of Binary White Dwarf Evolution", Odessa Astron. Publ., 14, 98-105 (2001). [ADS]. (Cited on page 82.)

[727] Solheim, J.-E. and Yungelson, L. R., "The White Dwarfs in AM CVn Systems - Candidates for SN Ia?", in Koester, D. and Moehler, S., eds., 14th European Workshop on White Dwarfs, Workshop in Kiel, Germany, 19 - 23 July 2004, ASP Conference Series, 334, p. 387, (Astronomical Society of the Pacific, San Francisco, 2005). [ADS]. (Cited on pages 64, 83, and 91.)

[728] Somiya, K., "Detector configuration of KAGRA-the Japanese cryogenic gravitational-wave detector", Class. Quantum Grav., 29, 124007 (2012). [DOI], [ADS], [arXiv:1111.7185 [gr-qc]]. (Cited on page 7.)

[729] Soraisam, M. and Gilfanov, M., "Constraint on Recurrent Novae as progenitors of Type Ia Supernovae", arXiv, e-print, (2014). [ADS], [arXiv:1401.6148 [astro-ph.SR]]. (Cited on page 68.)

[730] Sparks, W. M. and Stecher, T. P., "Supernova: The Result of the Death Spiral of a White Dwarf into a Red Giant", Astrophys. J., 188, 149 (1974). [DOI], [ADS]. (Cited on pages 36 and 77.)

[731] Spruit, H. C. and Phinney, E. S., "Birth kicks as the origin of pulsar rotation", Nature, 393, 139-141 (1998). [DOI]. (Cited on page 34.)

[732] Spruit, H. C. and Taam, R. E., "Circumbinary Disks and Cataclysmic Variable Evolution", Astrophys. J., 548, 900-907 (2001). [DOI], [ADS], [arXiv:astro-ph/0010194]. (Cited on page 29.)

[733] Stairs, I. H., "Testing General Relativity with Pulsar Timing", Living Rev. Relativity, 6, lrr-2003-5 (2003). [DOI], [ADS], [arXiv:astro-ph/0307536]. URL (accessed 15 January 2005): http://www.livingreviews.org/lrr-2003-5. (Cited on page 14.)

[734] Stairs, I. H., Thorsett, S. E. and Arzoumanian, Z., "Measurement of Gravitational Spin-Orbit Coupling in a Binary-Pulsar System", Phys. Rev. Lett., 93, 141101 (2004). [DOI], [ADS]. (Cited on page 34.)

[735] Stairs, I. H., Thorsett, S. E., Taylor, J. H. and Wolszczan, A., "Studies of the Relativistic Binary Pulsar PSR B1534+12. I. Timing Analysis", Astrophys. J., 581, 501-508 (2002). [DOI]. (Cited on page 15.)

[736] Starrfield, S., "On the cause of the nova outburst", Mon. Not. R. Astron. Soc., 152, 307-322 (1971). [ADS]. (Cited on page 60.)

[737] Starrfield, S., "On the cause of the nova outburst - II. Evolution at 1.00M๑", Mon. Not. R. Astron. Soc., 155, 129 (1971). [ADS]. (Cited on page 60.) 
[738] Starrfield, S., Sparks, W. M. and Truran, J. W., "CNO abundances and hydrodynamic models of the nova outburst. II - 1.00 solar mass models with enhanced carbon and oxygen", Astrophys. J. Suppl. Ser., 28, 247-270 (1974). [DOI], [ADS]. (Cited on page 37.)

[739] Starrfield, S., Truran, J. W., Sparks, W. M. and Kutter, G. S., "CNO Abundances and Hydrodynamic Models of the Nova Outburst", Astrophys. J., 176, 169 (1972). [DOI], [ADS]. (Cited on page 60.)

[740] Stasińska, G. et al., "The chemical composition of TS 01, the most oxygen-deficient planetary nebula. AGB nucleosynthesis in a metal-poor binary star", Astron. Astrophys., 511, A44 (2010). [DOI], [ADS]. (Cited on page 80.)

[741] Stephenson, F. R. and Green, D. A., Historical Supernovae and their Remnants, International Series on Astronomy and Astrophysics, 5, (Clarendon Press, Oxford, 2002). [ADS]. (Cited on page 10.)

[742] Sternberg, A. et al., "Multi-Epoch High-Spectral-Resolution Observations of Neutral Sodium in 14 Type Ia Supernovae", arXiv, e-print, (2013). [ADS], [arXiv:1311.3645 [astro-ph.HE]]. (Cited on page 70.)

[743] Stroeer, A., Heber, U., Lisker, T., Napiwotzki, R., Dreizler, S., Christlieb, N. and Reimers, D., "Hot subdwarfs from the ESO supernova Ia progenitor survey. II. Atmospheric parameters of subdwarf O stars", Astron. Astrophys., 462, 269-280 (2007). [DOI], [ADS], [arXiv:astro-ph/0609718]. (Cited on page 75.$)$

[744] Stroeer, A. and Vecchio, A., "The LISA verification binaries", Class. Quantum Grav., 23, S809-S818 (2006). [DOI], [ADS], [arXiv:astro-ph/0605227]. (Cited on pages 83 and 103.)

[745] Susa, H., "The Mass of the First Stars", Astrophys. J., 773, 185 (2013). [DOI]. (Cited on page 8.)

[746] Szypryt, P. et al., "Direct detection of SDSS J0926+3624 orbital expansion with ARCONS", Mon. Not. R. Astron. Soc., 439, 2765-2770 (2014). [DOI], [ADS], [arXiv:1309.4087 [astro-ph.SR]]. (Cited on page 85 .)

[747] Taam, R. E., "Hydrodynamical Investigations of Common Envelope Evolution", in Shara, M. M., ed., Stellar Collisions, Mergers and their Consequences, Proceedings of the conference held in New York, USA, 30 May-2 June, 2000, ASP Conference Series, 263, p. 81, (Astronomical Society of the Pacific, San Francisco, 2002). [ADS]. (Cited on page 39.)

[748] Taam, R. E. and Wade, R. A., "Angular momentum loss and the evolution of binaries of extreme mass ratio", Astrophys. J., 293, 504-507 (1985). [DOI], [ADS]. (Cited on page 85.)

[749] Takahashi, K., Yoshida, T. and Umeda, H., "Evolution of Progenitors for Electron Capture Supernovae", Astrophys. J., 771, 28 (2013). [DOI], [ADS], [arXiv:1302.6402 [astro-ph.SR]]. (Cited on page 9.)

[750] Takahashi, R. and Seto, N., "Parameter Estimation for Galactic Binaries by the Laser Interferometer Space Antenna", Astrophys. J., 575, 1030-1036 (2002). [DOI], [ADS]. (Cited on page 108.)

[751] Tanvir, N. R., Levan, A. J., Fruchter, A. S., Hjorth, J., Hounsell, R. A., Wiersema, K. and Tunnicliffe, R. L., "A 'kilonova' associated with the short-duration $\gamma$-ray burst GRB130603B", Nature, 500, 547549 (2013). [DOI], [ADS], [arXiv:1306.4971 [astro-ph.HE]]. (Cited on pages 7 and 109.)

[752] Taubenberger, S. et al., "High luminosity, slow ejecta and persistent carbon lines: SN 2009dc challenges thermonuclear explosion scenarios", Mon. Not. R. Astron. Soc., 412, 2735-2762 (2011). [DOI], [ADS], [arXiv:1011.5665 [astro-ph.SR]]. (Cited on page 73.)

[753] Tauris, T. M. and Takens, R. J., "Runaway velocities of stellar components originating from disrupted binaries via asymmetric supernova explosions", Astron. Astrophys., 330, 1047-1059 (1998). [ADS]. (Cited on page 32.) 
[754] Tauris, T. M. and van den Heuvel, E. P. J., "New Direct Observational Evidence for Kicks in SNe", in Kramer, M., Wex, N. and Wielebinski, R., eds., Pulsar Astronomy: 2000 and Beyond, Proceedings of IAU Colloquium 177 at the Max-Planck-Institut für Radioastronomie, Bonn, Germany, 30 August - 3 September 1999, ASP Conference Series, p. 595, (Astronomical Society of the Pacific, San Francisco, 2000). (Cited on page 34.)

[755] Tauris, T. M. and van den Heuvel, E. P. J., "Formation and evolution of compact stellar X-ray sources", in Lewin, W. H. G. and van der Klis, M., eds., Compact Stellar X-Ray Sources, Cambridge Astrophysics Series, 39, pp. 623-665, (Cambridge University Press, Cambridge; New York, 2006). [ADS]. (Cited on pages 18 and 21.)

[756] Tavani, M. et al., "Extreme particle acceleration in the microquasar CygnusX-3", Nature, 462, 620-623 (2009). [DOI], [ADS], [arXiv:0910.5344 [astro-ph.HE]]. (Cited on page 17.)

[757] Taylor, J. H. et al., "Binary pulsar", IAU Circ., 2704, 1 (1974). [ADS]. (Cited on pages 42 and 57.)

[758] Thorne, K. S., "Gravitational radiation", in Hawking, S. W. and Israel, W., eds., Three Hundred Years of Gravitation, pp. 330-458, (Cambridge University Press, Cambridge; New York, 1987). [Google Books]. (Cited on pages 7 and 54.)

[759] Thorne, K. S. and Zytkow, A. N., "Red giants and supergiants with degenerate neutron cores", Astrophys. J. Lett., 199, L19-L24 (1975). [DOI], [ADS]. (Cited on page 45.)

[760] Thorsett, S. E. and Chakrabarty, D., "Neutron Star Mass Measurements. I. Radio Pulsars", Astrophys. J., 512, 288-299 (1999). [DOI], [ADS]. (Cited on page 8.)

[761] Timmes, F. X., Woosley, S. E. and Weaver, T. A., "Galactic chemical evolution: Hydrogen through zinc", Astrophys. J. Suppl. Ser., 98, 617-658 (1995). [DOI], [ADS], [arXiv:astro-ph/9411003]. (Cited on page 7. )

[762] Timmes, F. X., Woosley, S. E. and Weaver, T. A., "The Neutron Star and Black Hole Initial Mass Function", Astrophys. J., 457, 834-843 (1996). [DOI]. (Cited on page 48.)

[763] Timpano, S. E., Rubbo, L. J. and Cornish, N. J., "Characterizing the galactic gravitational wave background with LISA", Phys. Rev. D, 73, 122001 (2006). [DOI], [ADS], [arXiv:gr-qc/0504071]. (Cited on page 97.)

[764] Tominaga, N., Blinnikov, S. I. and Nomoto, K., "Supernova Explosions of Super-asymptotic Giant Branch Stars: Multicolor Light Curves of Electron-capture Supernovae", Astrophys. J. Lett., 771, L12 (2013). [DOI], [ADS], [arXiv:1305.6813 [astro-ph.HE]]. (Cited on page 10.)

[765] Toonen, S. and Nelemans, G., "The effect of common-envelope evolution on the visible population of post-common-envelope binaries", Astron. Astrophys., 557, A87 (2013). [DOI], [ADS], [arXiv:1309.0327 [astro-ph.SR]]. (Cited on pages 39, 40, and 60.)

[766] Toonen, S., Nelemans, G. and Portegies Zwart, S., "Supernova Type Ia progenitors from merging double white dwarfs. Using a new population synthesis model", Astron. Astrophys., 546, A70 (2012). [DOI], [ADS], [arXiv:1208.6446 [astro-ph.HE]]. (Cited on pages 62 and 63.)

[767] Tornambé, A. and Piersanti, L., "Pre-explosive observational properties of Type Ia supernovae", Mon. Not. R. Astron. Soc., 431, 1812-1822 (2013). [DOI], [ADS], [arXiv:1304.7610 [astro-ph.GA]]. (Cited on pages 73 and 76.)

[768] Tovmassian, G. et al., "The Double-degenerate Nucleus of the Planetary Nebula TS 01: A Close Binary Evolution Showcase", Astrophys. J., 714, 178-193 (2010). [DOI], [ADS]. (Cited on page 80.)

[769] Tovmassian, G. H., Napiwotzki, R., Richer, M. G., Stasińska, G., Fullerton, A. W. and Rauch, T., "A Close Binary Nucleus in the MOST Oxygen-Poor Planetary Nebula PN G135.9+55.9", Astrophys. J., 616, 485-497 (2004). [DOI], [ADS]. (Cited on page 80.) 
[770] Townsley, D. M. and Bildsten, L., "Theoretical Modeling of the Thermal State of Accreting White Dwarfs Undergoing Classical Nova Cycles", Astrophys. J., 600, 390-403 (2004). [DOI], [ADS], [arXiv:astro-ph/0306080]. (Cited on page 60.)

[771] Townsley, D. M. and Gänsicke, B. T., "Cataclysmic Variable Primary Effective Temperatures: Constraints on Binary Angular Momentum Loss", Astrophys. J., 693, 1007-1021 (2009). [DOI], [ADS], [arXiv:0811.2447]. (Cited on page 26.)

[772] Trepl, L. et al., "Is there a compact companion orbiting the late O-type binary star HD 164816?", Mon. Not. R. Astron. Soc., 427, 1014-1023 (2012). [DOI], [ADS], [arXiv:1209.2592 [astro-ph.GA]]. (Cited on page 44.)

[773] Truran, J. W. and Glasner, S. A., "On the Nature of the Soft X-Ray Emission from Nova GQ Muscae 1983", in Bianchini, A., della Valle, M. and Orio, M., eds., Cataclysmic Variables, Proceedings of the conference held in Abano Terme, Italy, 20 - 24 June 1994, Astrophysics and Space Science Library, 205, p. 453, (Kluwer, Dordrecht, 1995). [ADS]. (Cited on page 61.)

[774] Truran, J. W. and Livio, M., "On the frequency of occurrence of oxygen-neon-magnesium white dwarfs in classical nova systems", Astrophys. J., 308, 721-727 (1986). [DOI], [ADS]. (Cited on page 10.)

[775] Tsugawa, M. and Osaki, Y., "Disk instability model for the AM Canum Venaticorum stars", Publ. Astron. Soc. Japan, 49, 75-84 (1997). [DOI], [ADS]. (Cited on page 106.)

[776] Tutukov, A. V. and Fedorova, A. V., "Formation and evolution of close binaries containing helium donors", Sov. Astron., 33, 606-613 (1989). (Cited on page 85.)

[777] Tutukov, A. V. and Fedorova, A. V., "Evolution of Close Stellar Binaries with Black Holes under the Action of Gravitational Radiation and Magnetic and Induced Stellar Winds of the Donor", Astron. Rep., 46, 765-778 (2002). [DOI], [ADS]. (Cited on page 46.)

[778] Tutukov, A. V., Fedorova, A. V., Ergma, E. V. and Yungelson, L. R., "Evolution of Low-Mass Close Binaries - The Minimum Orbital Period", Sov. Astron. Lett., 11, 52 (1985). [ADS]. (Cited on pages 79 and 82.)

[779] Tutukov, A. V., Fedorova, A. V., Ergma, E. V. and Yungelson, L. R., "The evolutionary status of MXB 1820-30 and other short-period low-mass X-ray sources", Sov. Astron. Lett., 13, 328 (1987). [ADS]. (Cited on page 82.)

[780] Tutukov, A. V., Fedorova, A. V. and Yungelson, L. R., "The evolution of dwarf binaries", Sov. Astron. Lett., 8, 365-370 (1982). (Cited on page 41.)

[781] Tutukov, A. V. and Yungelson, L. R., "Evolution of close binaries with mass loss from system I", Nauchn. Inform., 20, 86 (1971). [ADS]. (Cited on page 28.)

[782] Tutukov, A. V. and Yungelson, L. R., "Evolution of massive close binaries", Nauchn. Inform., 27, 70 (1973). [ADS]. (Cited on pages 38 and 42.)

[783] Tutukov, A. V. and Yungelson, L. R., "Evolution of massive common envelope binaries and mass loss", in de Loore, C. and Conti, P. S., eds., Mass loss and evolution of O-type stars, IAU Symposium 83 in Vancouver Island, Canada, 5 - 9 June, 1978, IAU Symposium, 83, p. 401, (D. Reidel, Dordrecht; Boston, 1979). (Cited on page 38.)

[784] Tutukov, A. V. and Yungelson, L. R., "On the influence of emission of gravitational waves on the evolution of low-mass close binary stars", Acta Astron., 29, 665-680 (1979). [ADS]. (Cited on pages $72,79,83,85$, and 92.)

[785] Tutukov, A. V. and Yungelson, L. R., "Evolutionary scenario for close binary systems of low and moderate masses", Nauchn. Inform., 49, 3 (1981). (Cited on pages 72 and 79.) 
[786] Tutukov, A. V. and Yungelson, L. R., "Duplicity of Hot Helium Subdwarfs", Sov. Astron. Lett., 34, 57 (1990). [ADS]. (Cited on pages 60 and 81.)

[787] Tutukov, A. V. and Yungelson, L. R., "Formation of neutron stars in binary systems", Astron. Rep., 37, 411-431 (1993). (Cited on pages 46 and 51.)

[788] Tutukov, A. V. and Yungelson, L. R., "The merger rate of neutron star and black hole binaries", Mon. Not. R. Astron. Soc., 260, 675-678 (1993). (Cited on pages 16, 46, 51, 52, and 55.)

[789] Tutukov, A. V. and Yungelson, L. R., "The merger rate of neutron star and black hole binaries", Mon. Not. R. Astron. Soc., 260, 675-678 (1993). [ADS]. (Cited on page 109.)

[790] Tutukov, A. V. and Yungelson, L. R., "Double-degenerate semidetached binaries with helium secondaries: cataclismic variables, supersoft X-ray sources, supernovae and accretion-induced collapses", Mon. Not. R. Astron. Soc., 280, 1035-1045 (1996). [DOI]. (Cited on pages 85 and 88.)

[791] Tutukov, A. V. and Yungelson, L. R., "A Model for the Population of Binary Stars in the Galaxy", Astron. Rep., 46, 667-683 (2002). [DOI], [ADS]. (Cited on pages 28, 30, 41, 50, 51, 52, and 95.)

[792] Tylenda, R. et al., "OGLE-2002-BLG-360: from a gravitational microlensing candidate to an overlooked red transient", Astron. Astrophys., 555, A16 (2013). [DOI], [ADS], [arXiv:1304.1694 [astroph.SR]]. (Cited on page 41.)

[793] Ulla, A., "The X-ray properties of AM Canum Venaticorum", Astron. Astrophys., 301, 469 (1995). [ADS]. (Cited on page 105.)

[794] van den Heuvel, E. P. J., "Late Stages of Close Binary Systems", in Eggleton, P., Mitton, S. and Whelan, J., eds., Structure and Evolution of Close Binary Systems, Proceedings of IAU Symposium 73 held in Cambridge, England, 28 July-1 August 1975, IAU Symposium, 73, pp. 35-62, (D. Reidel, Dordrecht; Boston, 1976). [ADS]. (Cited on page 38.)

[795] van den Heuvel, E. P. J., "Formation and evolution of X-ray binaries", in Lewin, W. H. G. and van den Heuvel, E. P. J., eds., Accretion-Driven Stellar X-ray Sources, Cambridge Astrophysics Series, 4, pp. 303-341, (Cambridge University Press, Cambridge; New York, 1983). (Cited on pages 21 and 30.)

[796] van den Heuvel, E. P. J., "X-Ray Binaries and Their Descendants: Binary Radio Pulsars; Evidence for Three Classes of Neutron Stars?", in Schönfelder, V., Lichti, G. and Winkler, C., eds., 5th INTEGRAL Workshop on the INTEGRAL Universe, Proceedings of the workshop held in Munich, Germany, 16 - 20 February 2004, Proceedings, SP-552, p. 185, (ESA Publications Division, Nordwijk, 2004). [ADS], [arXiv:astro-ph/0407451]. (Cited on page 42.)

[797] van den Heuvel, E. P. J., "The Formation and Evolution of Relativistic Binaries", in Colpi, M., Casella, P., Gorini, V., Moschella, U. and Possenti, A., eds., Physics of Relativistic Objects in Compact Binaries: From Birth to Coalescence, Astrophysics and Space Science Library, 359, pp. 125-198, (Springer, Berlin; New York, 2009). [DOI], [ADS], [Google Books]. (Cited on page 18.)

[798] van den Heuvel, E. P. J., "High space velocities of single radio pulsars versus low orbital eccentricities and masses of double neutron stars: Evidence for two different neutron star formation mechanisms", New Astron. Rev., 54, 140-144 (2010). [DOI], [ADS]. (Cited on page 10.)

[799] van den Heuvel, E. P. J., "Compact stars and the evolution of binary systems", Bull. Astr. Soc. India, 39, 1-20 (2011). [ADS]. (Cited on pages 21 and 42.)

[800] van den Heuvel, E. P. J., Bhattacharya, D., Nomoto, K. and Rappaport, S. A., "Accreting white dwarf models for CAL 83, CAL 87 and other ultrasoft X-ray sources in the LMC", Astron. Astrophys., 262, 97-105 (1992). [ADS]. (Cited on pages 60 and 72.)

[801] van den Heuvel, E. P. J. and de Loore, C., "The nature of X-ray binaries. III. Evolution of massive close binarieswith one collapsed component, with a possible application to Cygnus X3", Astron. Astrophys., 25, 387 (1973). (Cited on page 42.) 
[802] van den Heuvel, E. P. J. and Habets, G. M. H. J., "Observational lower mass limit for black hole formation derived from massive X-ray binaries", Nature, 309, 598-600 (1984). [DOI]. (Cited on page 48.)

[803] van der Sluys, M. V., Verbunt, F. and Pols, O. R., "Modelling the formation of double white dwarfs", Astron. Astrophys., 460, 209-228 (2006). [DOI], [ADS]. (Cited on page 40.)

[804] van Haaften, L. M., Nelemans, G., Voss, R., Toonen, S., Portegies Zwart, S. F., Yungelson, L. R. and van der Sluys, M. V., "Population synthesis of ultracompact X-ray binaries in the Galactic bulge", Astron. Astrophys., 552, A69 (2013). [DOI], [ADS], [arXiv:1302.7181 [astro-ph.SR]]. (Cited on pages 12 and 78.$)$

[805] van Haaften, L. M., Nelemans, G., Voss, R., Wood, M. A. and Kuijpers, J., "The evolution of ultracompact X-ray binaries", Astron. Astrophys., 537, A104 (2012). [DOI], [ADS], [arXiv:1111.5978 [astro-ph.SR]]. (Cited on page 78.)

[806] van Haaften, L. M., Voss, R. and Nelemans, G., "Long-term luminosity behavior of 14 ultracompact X-ray binaries", Astron. Astrophys., 543, A121 (2012). [DOI], [ADS], [arXiv:1206.0691 [astroph.HE]]. (Cited on page 78.)

[807] Vanbeveren, D., "Evolution of massive close binaries", Space Sci. Rev., 66, 327-347 (1993). [DOI], [ADS]. (Cited on page 46.)

[808] Vennes, S., Kawka, A., O'Toole, S. J., Németh, P. and Burton, D., "The Shortest Period sdB Plus White Dwarf Binary CD-30 11223 (GALEX J1411-3053)", Astrophys. J. Lett., 759, L25 (2012). [DOI], [ADS], [arXiv:1210.1512 [astro-ph.SR]]. (Cited on page 81.)

[809] Verbunt, F. and Rappaport, S., "Mass transfer instabilities due to angular momentum flows in close binaries", Astrophys. J., 332, 193-198 (1988). [DOI]. (Cited on pages 62 and 85.)

[810] Verbunt, F. and Zwaan, C., "Magnetic braking in low-mass X-ray binaries", Astron. Astrophys., 100, L7-L9 (1981). [ADS]. (Cited on pages 25 and 79.)

[811] Vereshchagin, S., Tutukov, A. V., Yungelson, L. R., Kraicheva, Z. and Popova, E., "Statistical study of visual binaries", Astrophys. Space Sci., 142, 245-254 (1988). [DOI]. (Cited on page 50.)

[812] Vila, S. C., "Late Evolution of Close Binaries", Astrophys. J., 168, 217-223 (1971). [DOI], [ADS]. (Cited on page 85.)

[813] Vink, J. S. et al., "Very Massive Stars (VMS) in the Local Universe", arXiv, e-print, (2013). [ADS], [arXiv:1302.2021 [astro-ph.SR]]. (Cited on page 8.)

[814] Voss, B., Koester, D., Napiwotzki, R., Christlieb, N. and Reimers, D., "High-resolution UVES/VLT spectra of white dwarfs observed for the ESO SN Ia progenitor survey. II. DB and DBA stars", Astron. Astrophys., 470, 1079-1088 (2007). [DOI], [ADS]. (Cited on page 79.)

[815] Voss, R. and Tauris, T. M., "Galactic distribution of merging neutron stars and black holes prospects for short gamma-ray burst progenitors and LIGO/VIRGO", Mon. Not. R. Astron. Soc., 342, 1169-1184 (2003). [DOI], [ADS], [arXiv:astro-ph/0303227 [astro-ph]]. (Cited on pages 46, 52, and 53.)

[816] Vranesevic, N. et al., "Pulsar Birthrates from the Parkes Multibeam Survey", Astrophys. J. Lett., 617, L139-L142 (2004). [DOI], [ADS], [astro-ph/0310201]. (Cited on page 50.)

[817] Wade, R. A., "A double grid of accretion disc model spectra for cataclysmic variable stars", Mon. Not. R. Astron. Soc., 208, 381-398 (1984). [ADS]. (Cited on page 105.) 
[818] Waldman, R., Sauer, D., Livne, E., Perets, H., Glasner, A., Mazzali, P. A., Truran, J. W. and Gal-Yam, A., "Helium Shell Detonations on Low-mass White Dwarfs as a Possible Explanation for SN 2005E", Astrophys. J., 738, 21 (2011). [DOI], [ADS], [arXiv:1009.3829 [astro-ph.SR]]. (Cited on page 74.)

[819] Wang, B., Meng, X., Chen, X. and Han, Z., "The helium star donor channel for the progenitors of Type Ia supernovae", Mon. Not. R. Astron. Soc., 395, 847-854 (2009). [DOI], [ADS], [arXiv:0901.3496 [astro-ph.SR]]. (Cited on page 64.)

[820] Wang, X., Wang, L., Filippenko, A. V., Zhang, T. and Zhao, X., "Evidence for Two Distinct Populations of Type Ia Supernovae", Science, 340, 170-173 (2013). [DOI], [ADS], [arXiv:1303.2601 [astro-ph.CO]]. (Cited on page 7.)

[821] Warner, B., Cataclysmic Variable Stars, Cambridge Astrophysics Series, 28, (Cambridge University Press, Cambridge; New York, 1995). [ADS]. (Cited on pages 60 and 79.)

[822] Warner, B. and Robinson, E. L., "Observations of rapid blue variables - IX. AM CVn (HZ 29)", Mon. Not. R. Astron. Soc., 159, 101-111 (1972). [ADS]. (Cited on page 57.)

[823] Webbink, R. F., "The evolution of low-mass close binary systems. IV. 0.80M $\odot+0.40 M_{\odot}$ : Catastrophic mass loss", Astrophys. J., 211, 881-889 (1977). [DOI], [ADS]. (Cited on page 36.)

[824] Webbink, R. F., "The formation of the white dwarfs in close binary systems", in van Horn, H. M. and Weidemann, V., eds., White Dwarfs and Variable Degenerate Stars, Proceedings of the Colloquium, July 30 - August 21979 and Fourth Annual Workshop on Novae, Dwarf Novae and Other Cataclysmic Variables at the University of Rochester in Rochester, N. Y., 3 August 1979, IAU Colloquium, 53, pp. 426-447, (University of Rochester, Rochester, NY, 1979). (Cited on page 79.)

[825] Webbink, R. F., "Double white dwarfs as progenitors of R Coronae Borealis stars and Type I supernovae", Astrophys. J., 277, 355-360 (1984). [DOI], [ADS]. (Cited on pages 38, 64, 72, 76, and 79.)

[826] Webbink, R. F., "Common Envelope Evolution Redux", in Milone, E. F., Leahy, D. A. and Hobill, D. W., eds., Short-Period Binary Stars: Observations, Analyses, and Results, Astrophysics and Space Science Library, 352, p. 233, (Springer, Berlin; New York, 2008). [ADS], [arXiv:0704.0280], [Google Books]. (Cited on page 28.)

[827] Webbink, R. F., "Watch This Space: Observing Merging White Dwarfs", in Kologera, V. and van der Sluys, M., eds., International Conference on Binaries: In celebration of Ron Webbink's 65th Birthday, Mykonos, Greece, 22 - 25 June 2010, AIP Conference Proceedings, 1314, pp. 217-222, (American Institute of Physics, Melville, 2010). [DOI], [ADS], [arXiv:1011.6317 [astro-ph.SR]]. (Cited on page 94.)

[828] Webbink, R. F. and Han, Z., "Gravitational Radiation from Close Double White Dwarfs", in Folkner, W. M., ed., Laser Interferometer Space Antenna, Second International LISA Symposium on the Detection and Observation of Gravitational Waves in Space, Pasadena, California, July 1998, AIP Conference Proceedings, 456, p. 61, (American Institure of Physics, Woodbury, NY, 1998). [ADS]. (Cited on pages 93 and 97.)

[829] Webbink, R. F. and Iben Jr, I., "Tidal interaction and coalescence of close binary white dwarfs", in Philip, A. G. D., Hayes, D. S. and Liebert, J. W., eds., The Second Conference on Faint Blue Stars (IAU Colloquium 95), Conference in Tucson, Arizona, 1 - 5 June 1987, pp. 445-456, (L. Davis Press, Schenectady, NY, 1987). [ADS]. (Cited on pages 84 and 94.)

[830] Weidner, C., Pflamm-Altenburg, J. and Kroupa, P., "The Galaxy-wide IMF - from Star Clusters to Galaxies", in Treyer, M., Wyder, T., Neill, J., Seibert, M. and Lee, J., eds., UP2010: Have Observations Revealed a Variable Upper End of the Initial Mass Function?, Proceedings of a Conference held at Sedona, Arizona, USA 20 - 25 June 2010, ASP Conference Series, 440, p. 19, (Astronomical 
Society of the Pacific, San Francisco, 2011). [ADS], [arXiv:1011.1905 [astro-ph.GA]]. (Cited on page 9.)

[831] Weisberg, J. M. and Taylor, J. H., "General Relativistic Geodetic Spin Precession in Binary Pulsar B1913+16: Mapping the Emission Beam in Two Dimensions", Astrophys. J., 576, 942-949 (2002). [DOI]. (Cited on page 34.)

[832] Weisberg, J. M. and Taylor, J. H., "The Relativistic Binary Pulsar B1913+16: Thirty Years of Observations and Analysis", in Rasio, F. A. and Stairs, I. H., eds., Binary Radio Pulsars, Proceedings of a meeting held at the Aspen Center for Physics, Colorado, USA, 12 -16 January 2004, ASP Conference Series, 328, p. 25, (Astronomical Society of the Pacific, San Francisco, 2005). [ADS], [arXiv:astro-ph/0407149]. (Cited on page 57.)

[833] Wesson, R. et al., "A Planetary Nebula around Nova V458 Vulpeculae Undergoing Flash Ionization", Astrophys. J. Lett., 688, L21-L24 (2008). [DOI], [ADS]. (Cited on page 80.)

[834] Wettig, T. and Brown, G. E., "The evolution of relativistic binary pulsars", New Astronomy, 1, 17-34 (1996). [DOI]. (Cited on page 46.)

[835] Wex, N., Kalogera, V. and Kramer, M., "Constraints on Supernova Kicks from the Double Neutron Star System PSR B1913+16”, Astrophys. J., 528, 401-409 (2000). [DOI]. (Cited on page 34.)

[836] Whelan, J. and Iben Jr, I., "Binaries and Supernovae of Type I", Astrophys. J., 186, 1007-1014 (1973). [DOI], [ADS]. (Cited on page 61.)

[837] Whyte, C. A. and Eggleton, P. P., "Comments on the evolution and origin of cataclysmic binaries", Mon. Not. R. Astron. Soc., 190, 801-823 (1980). [ADS]. (Cited on page 79.)

[838] Willems, B., Henninger, M., Levin, T., Ivanova, N., Kalogera, V., McGhee, K., Timmes, F. X. and Fryer, C. L., "Understanding Compact Object Formation and Natal Kicks. I. Calculation Methods and the Case of GRO J1655-40", Astrophys. J., 625, 324-346 (2005). [DOI]. (Cited on page 35.)

[839] Willems, B. and Kalogera, V., "Constraints on the Formation of PSR J0737-3039: The Most Probable Isotropic Kick Magnitude", Astrophys. J. Lett., 603, L101-L104 (2004). [DOI]. (Cited on pages 34 and 46.)

[840] Willems, B., Kalogera, V. and Henninger, M., "Pulsar Kicks and Spin Tilts in the Close Double Neutron Stars PSR J0737-3039, PSR B1534+12, and PSR B1913+16”, Astrophys. J., 616, 414-438 (2004). [DOI]. (Cited on page 34.)

[841] Willems, B., Kolb, U., Sandquist, E. L., Taam, R. E. and Dubus, G., "Angular Momentum Losses and the Orbital Period Distribution of Cataclysmic Variables below the Period Gap: Effects of Circumbinary Disks", Astrophys. J., 635, 1263-1280 (2005). [DOI], [ADS], [arXiv:astro-ph/0509355]. (Cited on page 26.)

[842] Williams, R. E. and Ferguson, D. H., "He I line emission and the helium abundance in cataclysmic variables", Astrophys. J., 257, 672-685 (1982). [DOI], [ADS]. (Cited on page 82.)

[843] Wolf, W. M., Bildsten, L., Brooks, J. and Paxton, B., "Hydrogen Burning on Accreting White Dwarfs: Stability, Recurrent Novae, and the Post-nova Supersoft Phase", Astrophys. J., 777, 136 (2013). [DOI], [ADS], [arXiv:1309.3375 [astro-ph.SR]]. (Cited on pages 60 and 61.)

[844] Wolszczan, A., "PSR 1257+12 and PSR 1534+12", IAU Circ., 1990(5073) (1990). [ADS]. Online version (accessed 18 March 2014):

http://www.cbat.eps.harvard.edu/iauc/05000/05073.html. (Cited on page 15.)

[845] Wong, T.-W., Valsecchi, F., Fragos, T. and Kalogera, V., "Understanding Compact Object Formation and Natal Kicks. III. The Case of Cygnus X-1", Astrophys. J., 747, 111 (2012). [DOI], [ADS], [arXiv:1107.5585 [astro-ph.HE]]. (Cited on page 35.) 
[846] Wongwathanarat, A., Janka, H.-T. and Müller, E., "Three-dimensional neutrino-driven supernovae: Neutron star kicks, spins, and asymmetric ejection of nucleosynthesis products", Astron. Astrophys., 552, A126 (2013). [DOI], [ADS], [arXiv:1210.8148 [astro-ph.HE]]. (Cited on page 34.)

[847] Woods, T. E. and Gilfanov, M., "He II recombination lines as a test of the nature of SN Ia progenitors in elliptical galaxies", Mon. Not. R. Astron. Soc., 432, 1640-1650 (2013). [DOI], [ADS], [arXiv:1302.5911 [astro-ph.SR]]. (Cited on page 72.)

[848] Woods, T. E. and Ivanova, N., "Can We Trust Models for Adiabatic Mass Loss?", Astrophys. J. Lett., 739, L48 (2011). [DOI], [ADS], [arXiv:1108.2752 [astro-ph.SR]]. (Cited on page 41.)

[849] Woods, T. E., Ivanova, N., van der Sluys, M. V. and Chaichenets, S., "On the Formation of Double White Dwarfs through Stable Mass Transfer and a Common Envelope", Astrophys. J., 744, 12 (2012). [DOI], [ADS], [arXiv:1102.1039 [astro-ph.SR]]. (Cited on pages 40 and 41.)

[850] Woosley, S. E. and Heger, A., "The Progenitor Stars of Gamma-Ray Bursts", Astrophys. J., 637, 914-921 (2006). [DOI], [ADS]. (Cited on page 11.)

[851] Woosley, S. E., Heger, A. and Weaver, T. A., "The evolution and explosion of massive stars", Rev. Mod. Phys., 74, 1015-1071 (2002). [DOI], [ADS]. (Cited on page 17.)

[852] Woosley, S. E., Langer, N. and Weaver, T. A., "The Presupernova Evolution and Explosion of Helium Stars That Experience Mass Loss", Astrophys. J., 448, 315 (1995). [DOI]. (Cited on page 48.)

[853] Woudt, P. A. and Steeghs, D., "Spectroscopy and Near-Infrared Photometry of the Helium Nova V445 Puppis", in Burderi, L., Antonelli, L. A., D’Antona, F., Di Salvo, T., Israel, G. L., Piersanti, L., Tornambè, A. and Straniero, O., eds., Interacting Binaries: Accretion, Evolution, and Outcomes, Proceedings of conference held in Cefalu, Sicily, Italy, 4-10 July 2004, AIP Conference Proceedings, 797, pp. 647-650, (American Institute of Physics, Melville, 2005). [DOI], [ADS]. (Cited on page 91.)

[854] Woudt, P. A. et al., "The Expanding Bipolar Shell of the Helium Nova V445 Puppis", Astrophys. $J ., \mathbf{7 0 6}, 738-746$ (2009). [DOI], [ADS]. (Cited on page 91.)

[855] Xu, X.-J. and Li, X.-D., "Erratum: 'On the Binding Energy Parameter $\lambda$ of Common Envelope Evolution' (2010, ApJ, 716, 114)", Astrophys. J., 722, 1985-1988 (2010). [DOI], [ADS]. (Cited on page 39.)

[856] Xu, X.-J. and Li, X.-D., "On the Binding Energy Parameter $\lambda$ of Common Envelope Evolution", $A s-$ trophys. J., 716, 114-121 (2010). [DOI], [ADS], [arXiv:1004.4957 [astro-ph.SR]]. (Cited on page 39.)

[857] Yagi, K., "Scientific Potential of Decigo Pathfinder and Testing GR with Space-Borne Gravitational Wave Interferometers", Int. J. Mod. Phys. D, 22, 1341013 (2013). [DOI], [ADS], [arXiv:1302.2388 [gr-qc]]. (Cited on page 7.)

[858] Yamanaka, M. et al., "Early Phase Observations of Extremely Luminous Type Ia Supernova 2009dc", Astrophys. J. Lett., 707, L118-L122 (2009). [DOI], [ADS]. (Cited on page 73.)

[859] Yaron, O., Prialnik, D., Shara, M. M. and Kovetz, A., "An Extended Grid of Nova Models. II. The Parameter Space of Nova Outbursts", Astrophys. J., 623, 398-410 (2005). [DOI], [ADS]. (Cited on pages 60,67 , and 68.)

[860] Yoon, S.-C. and Langer, N., "The first binary star evolution model producing a Chandrasekhar mass white dwarf", Astron. Astrophys., 412, L53-L56 (2003). [DOI], [ADS]. (Cited on pages 64 and 77.)

[861] Yoon, S.-C. and Langer, N., "Presupernova evolution of accreting white dwarfs with rotation", Astron. Astrophys., 419, 623-644 (2004). [DOI], [ADS], [astro-ph/0402287]. (Cited on page 69.)

[862] Yoon, S.-C. and Langer, N., "On the evolution of rapidly rotating massive white dwarfs towards supernovae or collapses", Astron. Astrophys., 435, 967-985 (2005). [DOI], [ADS], [astro-ph/0502133]. (Cited on page 69.) 
[863] Yoon, S.-C., Podsiadlowski, P. and Rosswog, S., "Remnant evolution after a carbon-oxygen white dwarf merger", Mon. Not. R. Astron. Soc., 380, 933-948 (2007). [DOI], [ADS]. (Cited on pages 73 and 76.)

[864] Yu, S. and Jeffery, C. S., "The gravitational wave signal from diverse populations of double white dwarf binaries in the Galaxy", Astron. Astrophys., 521, A85 (2010). [DOI], [arXiv:1007.4267 [astroph.SR]]. (Cited on pages 93, 95, and 97.)

[865] Yu, S. and Jeffery, C. S., "The influence of star formation history on the gravitational wave signal from close double degenerates in the thin disc", Mon. Not. R. Astron. Soc., 429, 1602-1608 (2013). [DOI], [ADS], [arXiv:1211.5091 [astro-ph.GA]]. (Cited on pages 93 and 95.)

[866] Yuan, F. et al., "The Exceptionally Luminous Type Ia Supernova 2007if", Astrophys. J., 715, 13381343 (2010). [DOI], [ADS]. (Cited on page 73.)

[867] Yungelson, L. R., "Evolution of close binaries with mass loss from the system. III. Systems containing white dwarfs", Nauchn. Inform., 26, 71-82 (1973). [ADS]. In Russian. (Cited on page 31.)

[868] Yungelson, L. R., "Evolution of the secondary component of a close binary system", Nauchn. Inform., 27, 93 (1973). [ADS]. (Cited on page 36.)

[869] Yungelson, L. R., "Population synthesis for low and intermediate mass binaries", in Burderi, L., Antonelli, L. A., D’Antona, F., Di Salvo, T., Israel, G. L., Piersanti, L., Tornambè, A. and Straniero, O., eds., Interacting Binaries: Accretion, Evolution, and Outcomes, Proceedings of conference held in Cefalù, Sicily, 4-10 July 2004, AIP Conference Proceedings, 797, pp. 1-10, (American Institute of Physics, Melville, 2005). [DOI], [ADS], [arXiv:astro-ph/0412635]. (Cited on pages 52 and 60.)

[870] Yungelson, L. R., "Evolution of low-mass helium stars in semidetached binaries", Astron. Lett., 34, 620-634 (2008). [DOI], [ADS], [arXiv:0804.2780]. (Cited on pages 64, 88, and 89.)

[871] Yungelson, L. R., "Evolution of the number of accreting white dwarfs with shell nuclear burning and the SNe Ia rate", Astron. Lett., 36, 780-787 (2010). [DOI], [ADS], [arXiv:1006.4451 [astro-ph.HE]]. (Cited on pages 7,61, and 67.)

[872] Yungelson, L. R., Lasota, J.-P., Nelemans, G., Dubus, G., van den Heuvel, E. P. J., Dewi, J. and Portegies Zwart, S. F., "The origin and fate of short-period low-mass black-hole binaries", Astron. Astrophys., 454, 559-569 (2006). [DOI], [ADS]. (Cited on pages 35 and 52.)

[873] Yungelson, L. R. and Livio, M., "Type Ia Supernovae: An Examination of Potential Progenitors and the Redshift Distribution", Astrophys. J., 497, 168 (1998). [DOI]. (Cited on pages 7, 41, and 68.)

[874] Yungelson, L. R. and Livio, M., "Supernova Rates: A Cosmic History", Astrophys. J., 528, 108-117 (2000). [DOI]. (Cited on page 7.)

[875] Yungelson, L. R., Livio, M., Truran, J. W., Tutukov, A. and Fedorova, A., "A Model for the Galactic Population of Binary Supersoft X-Ray Sources", Astrophys. J., 466, 890 (1996). [DOI]. (Cited on page 61.)

[876] Yungelson, L. R., Livio, M., Tutukov, A. and Kenyon, S. J., "A Model for the Galactic Population of Symbiotic Stars with White Dwarf Accretors", Astrophys. J., 447, 656 (1995). [DOI]. (Cited on page 67.)

[877] Yungelson, L. R., Nelemans, G. and van den Heuvel, E. P. J., "On the formation of neon-enriched donor stars in ultracompact X-ray binaries", Astron. Astrophys., 388, 546-551 (2002). [DOI]. (Cited on pages 31,89 , and 95 .)

[878] Yungelson, L. R. and Portegies Zwart, S. F., "Evolution of Close Binaries: Formation and Merger of Neutron Star Binaries", in Davier, M. and Hello, P., eds., Second Workshop on Gravitational Wave Data Analysis, Orsay, France, 13-15 November 1997, pp. 77-86, (Éditions Frontièrs, Paris, 1998). [ADS], [arXiv:astro-ph/9801127], [Google Books]. (Cited on page 44.) 
[879] Yungelson, L. R. and Tutukov, A. V., "A Model for the Population of Helium Stars in the Galaxy: Low-Mass Stars", Astron. Rep., 49, 871-883 (2005). [DOI], [ADS]. (Cited on page 60.)

[880] Yungelson, L. R., van den Heuvel, E. P. J., Vink, J. S., Portegies Zwart, S. F. and de Koter, A., "On the evolution and fate of super-massive stars", Astron. Astrophys., 477, 223-237 (2008). [DOI], [ADS], [arXiv:0710.1181]. (Cited on page 8.)

[881] Yusof, N. et al., "Evolution and fate of very massive stars", Mon. Not. R. Astron. Soc., 433, 11141132 (2013). [DOI], [ADS], [arXiv:1305.2099 [astro-ph.SR]]. (Cited on page 12.)

[882] Zapolsky, H. S. and Salpeter, E. E., "The Mass-Radius Relation for Cold Spheres of Low Mass", Astrophys. J., 158, 809 (1969). [DOI], [ADS]. (Cited on page 85.)

[883] Zdziarski, A. A., Mikołajewska, J. and Belczyński, K., "Cyg X-3: a low-mass black hole or a neutron star", Mon. Not. R. Astron. Soc., 429, L104-L108 (2013). [DOI], [ADS], [arXiv:1208.5455 [astroph.HE]]. (Cited on page 19.)

[884] Zel'dovich, Y. B., Ivanova, L. N. and Nadezhin, D. K., "Nonstationary Hydrodynamical Accretion onto a Neutron Star", Sov. Astron., 16, 209 (1972). [ADS]. (Cited on page 45.)

[885] Zhang, X. and Jeffery, C. S., "Can R Coronae Borealis stars form from the merger of two helium white dwarfs?", Mon. Not. R. Astron. Soc., 426, L81-L85 (2012). [DOI], [ADS], [arXiv:1208.3907 [astro-ph.SR]]. (Cited on page 64.)

[886] Zorotovic, M., Schreiber, M. R., Gänsicke, B. T. and Nebot Gómez-Morán, A., "Post-commonenvelope binaries from SDSS. IX: Constraining the common-envelope efficiency", Astron. Astrophys., 520, A86 (2010). [DOI], [ADS], [arXiv:1006.1621 [astro-ph.SR]]. (Cited on page 40.)

[887] Zorotovic, M. et al., "Post common envelope binaries from SDSS. XIII. Mass dependencies of the orbital period distribution", Astron. Astrophys., 536, L3 (2011). [DOI], [ADS], [arXiv:1111.2512 [astro-ph.SR]]. (Cited on page 60.) 